\title{
Synthesis of (Z)- $\alpha$-Trifluoromethyl Alkenyl Triflate: a Scaffold for Diverse Trifluoromethylated Species
}

\author{
Yilong Zhao, $\uparrow$ Yuhan Zhou, ${ }^{*} \dagger$ Juan Liu, $\uparrow$ Dongmei Yang, $\uparrow$ Liang Tao, $\uparrow$ Yang Liu, $\uparrow$ Xiaoliang Dong, $\uparrow$ Jianhui Liu, $\uparrow$ Jingping Qu $\uparrow$ \\ † State Key Laboratory of Fine Chemicals, School of Pharmaceutical Science and Technology, Dalian University of Technology, Dalian 116024, P.R. China \\ tSchool of Petroleum and Chemical Engineering, Dalian University of Technology, Panjin 124221, China \\ *E-mail: zhouyh@dl.cn (Y. Zhou)
}

\section{Supporting Information}

\section{Contents}

The Crystal Structure Determination

${ }^{1} \mathrm{H},{ }^{13} \mathrm{C}$ and ${ }^{19} \mathrm{~F}$ NMR spectra for $2 \mathrm{a}-2 \mathrm{q}$

${ }^{1} \mathrm{H},{ }^{13} \mathrm{C}$ and ${ }^{19} \mathrm{~F}$ NMR spectra for $3 \mathrm{a}-3 \mathrm{~s}$

S57-S113

${ }^{1} \mathrm{H},{ }^{13} \mathrm{C}$ and ${ }^{19} \mathrm{~F}$ NMR spectra for $4 \mathrm{a}-4 \mathrm{j}$

${ }^{1} \mathrm{H}$ NMR spectra for $5 \mathbf{a}-\mathbf{5 d}$

S144-S147

${ }^{1} \mathrm{H},{ }^{13} \mathrm{C}$ and ${ }^{19} \mathrm{~F}$ NMR spectra for $5 e$

S148-S150

${ }^{1}$ H NMR spectra for $6 a-6 d$

S151-S154

${ }^{1} \mathrm{H},{ }^{13} \mathrm{C}$ and ${ }^{19} \mathrm{~F}$ NMR spectra for $2 \mathrm{r}, 3 \mathrm{t}, 7$

S155-S163 


\section{The Crystal Structure Determination}

The crystals suitable for X-ray crystallography were obtained by slow evaporation of solvent of the solution of the substances in $n$-hexane at room temperature. The data were obtained on a CCD diffractometer with graphite-monochromated. Mo $\mathrm{K} \alpha$ radiation $(\lambda=0.71073 \AA)$. Empirical absorption corrections were performed using the SADABS program. ${ }^{1}$ Structures were solved by direct methods and were refined by full-matrix least-squares based on all data using $F^{2}$ in Shelx97., ${ }^{2,3}$ All of the non-hydrogen atoms were refined anisotropically. All of the hydrogen atoms were generated and refined in ideal positions. Crystal data and collection details for $\mathbf{2 e}$ and $\mathbf{3 q}$ are given in Table S1.

Table S1. Crystal data and structure refinement for complexes $\mathbf{2 e}$ and $\mathbf{3 q}$

\begin{tabular}{lll}
\hline & \multicolumn{1}{c}{$2 \mathbf{e}$} & \multicolumn{1}{c}{$3 \mathbf{q}$} \\
\hline Formula & $\mathrm{C}_{10} \mathrm{H}_{5} \mathrm{BrF}_{6} \mathrm{O}_{3} \mathrm{~S}$ & $\mathrm{C}_{16} \mathrm{H}_{12} \mathrm{ClF}_{3}$ \\
Formula weight & 399.11 & 296.71 \\
Crystal dimensions $\left(\mathrm{mm}^{3}\right)$ & $0.33 \times 0.28 \times 0.23$ & $0.35 \times 0.30 \times 0.35$ \\
Crystal system & Triclinic & Orthorhombic \\
Space group & $\mathrm{P}-1$ & $\mathrm{P} 2(1) 2(1) 2(1)$ \\
$\mathrm{a}(\AA)$ & $7.8097(5)$ & $5.892(5)$ \\
$\mathrm{b}(\AA)$ & $8.4203(5)$ & $11.809(10)$ \\
$\mathrm{c}(\AA)$ & $10.7272(6)$ & $20.667(18)$ \\
$\alpha\left(^{\circ}\right)$ & $98.112(4)$ & 90.00 \\
$\beta\left(^{\circ}\right)$ & $95.627(4)$ & 90.00 \\
$\gamma\left({ }^{\circ}\right)$ & $98.351(4)$ & 90.00 \\
Volume $\left(\AA^{3}\right)$ & $685.88(7)$ & $1438(2)$ \\
$Z$ & 2 & 4 \\
$T(\mathrm{~K})$ & $296(2)$ & $300(2)$ \\
$D$ calcd $(\mathrm{g}$ cm & -3 & 1.371 \\
$\left.\mu(\mathrm{mm})^{-1}\right)$ & 1.933 & 0.285 \\
$F(000)$ & 3.224 & 608 \\
No. of rflns. collected & 388 & 12129 \\
No. of indep. rflns. $/ R_{\text {int }}$ & 7205 & $2469 / 0.1061$ \\
No. of obsd. rflns. $\left[I_{0}>2 \sigma\left(I_{0}\right)\right]$ & $2413 / 0.0460$ & 1715 \\
Data / restraints / parameters & 1928 & $2469 / 0 / 182$ \\
$R_{I} / w R_{2}\left[I_{0}>2 \sigma\left(I_{0}\right)\right]$ & $2413 / 0 / 191$ & $0.0549 / 0.1386$ \\
$R_{I} / w R_{2}($ all data $)$ & $0.0421 / 0.1277$ & $0.0950 / 0.1527$ \\
$\mathrm{GOF}\left(\right.$ on $\left.F^{2}\right)$ & $0.0523 / 0.1340$ & 1.002 \\
Largest diff. peak and hole $\left(\mathrm{e} \AA^{-3}\right)$ & 0.971 & $0.166 /-0.147$ \\
\hline
\end{tabular}

\section{REFERENCES}

(1) Sheldrich, G. M. SADABS, Program for empirical absorption correction, University of Göttingen: Germany, 1996.

(2) Sheldrich, G. M. SHELX-97, program for crystal structure determination, University of Göttingen: Germany, 1997. 
(3) Sheldrick, G. M. SHELXL-97, Program for refinement of crystal structures, University of Göttingen: Germany, 1997. 


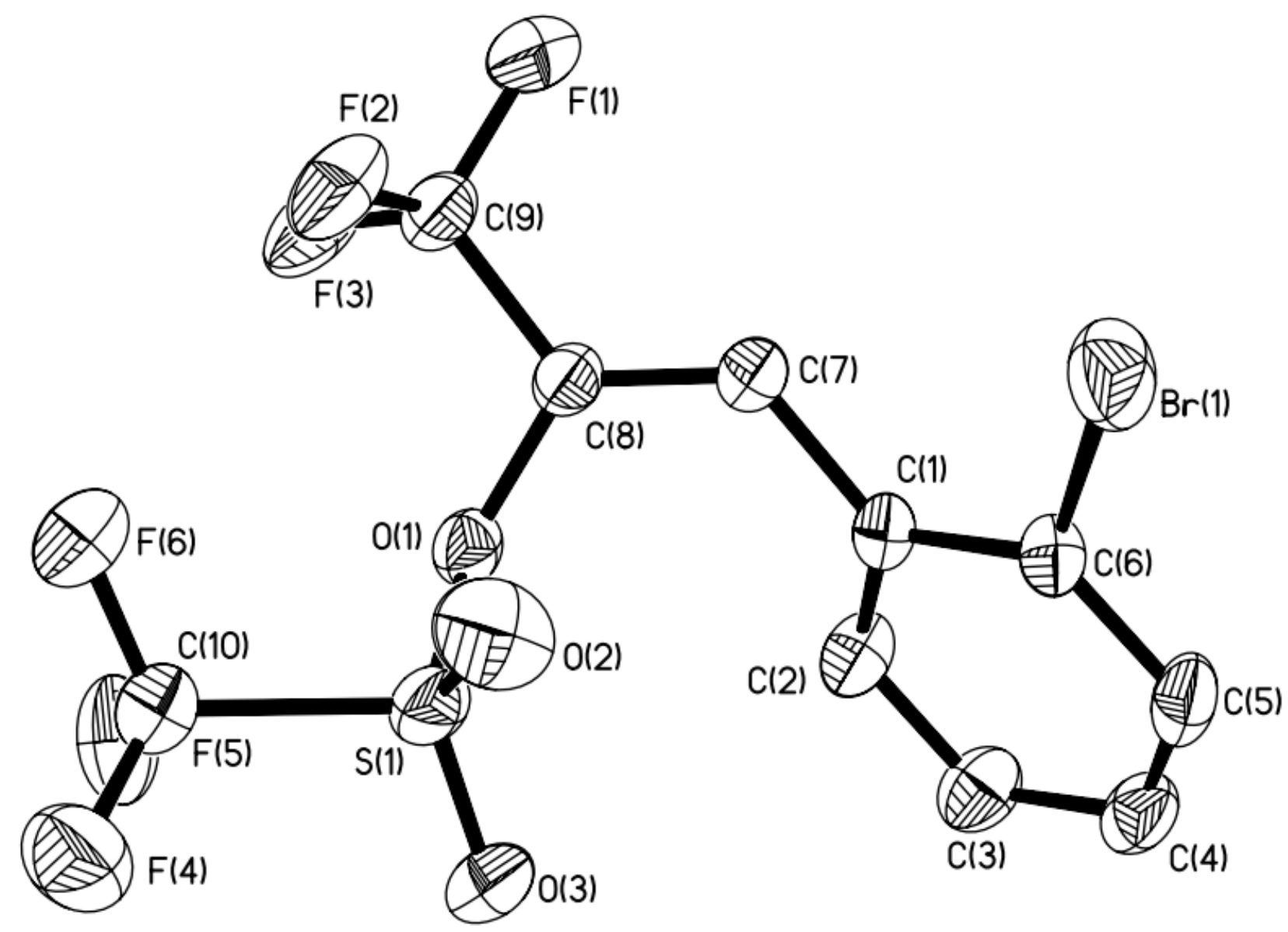

Figure S1 ORTEP diagram of the single-crystal X-ray structure of compound $\mathbf{2 e}$ (Hydrogen atoms are omitted for clarity and thermal ellipsoids shown at $30 \%$ probability) 


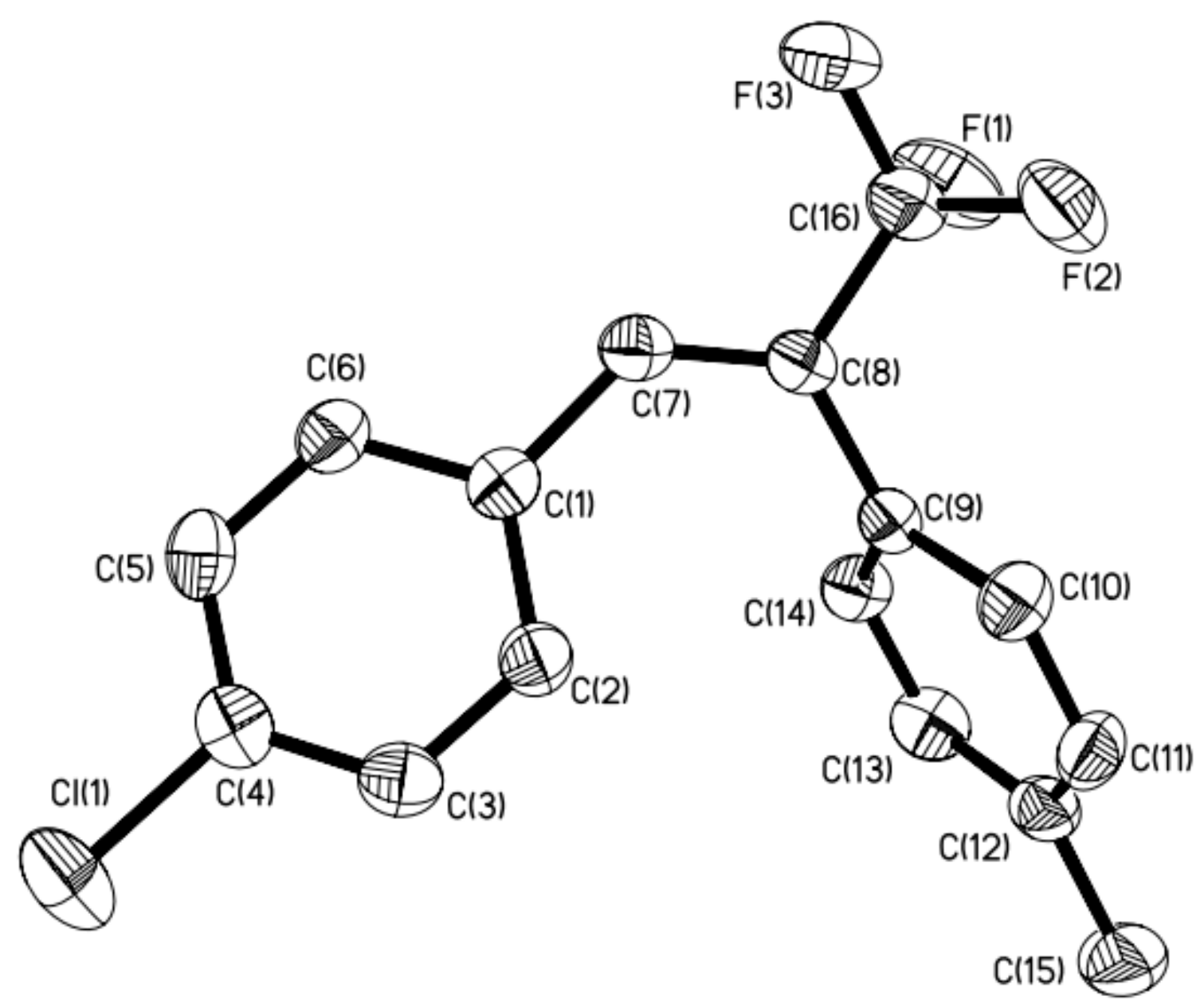

Figure S2 ORTEP diagram of the single-crystal X-ray structure of compound 3q (Hydrogen atoms are omitted for clarity and thermal ellipsoids shown at 30\% probability) 
หู่

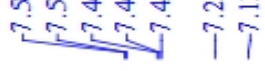
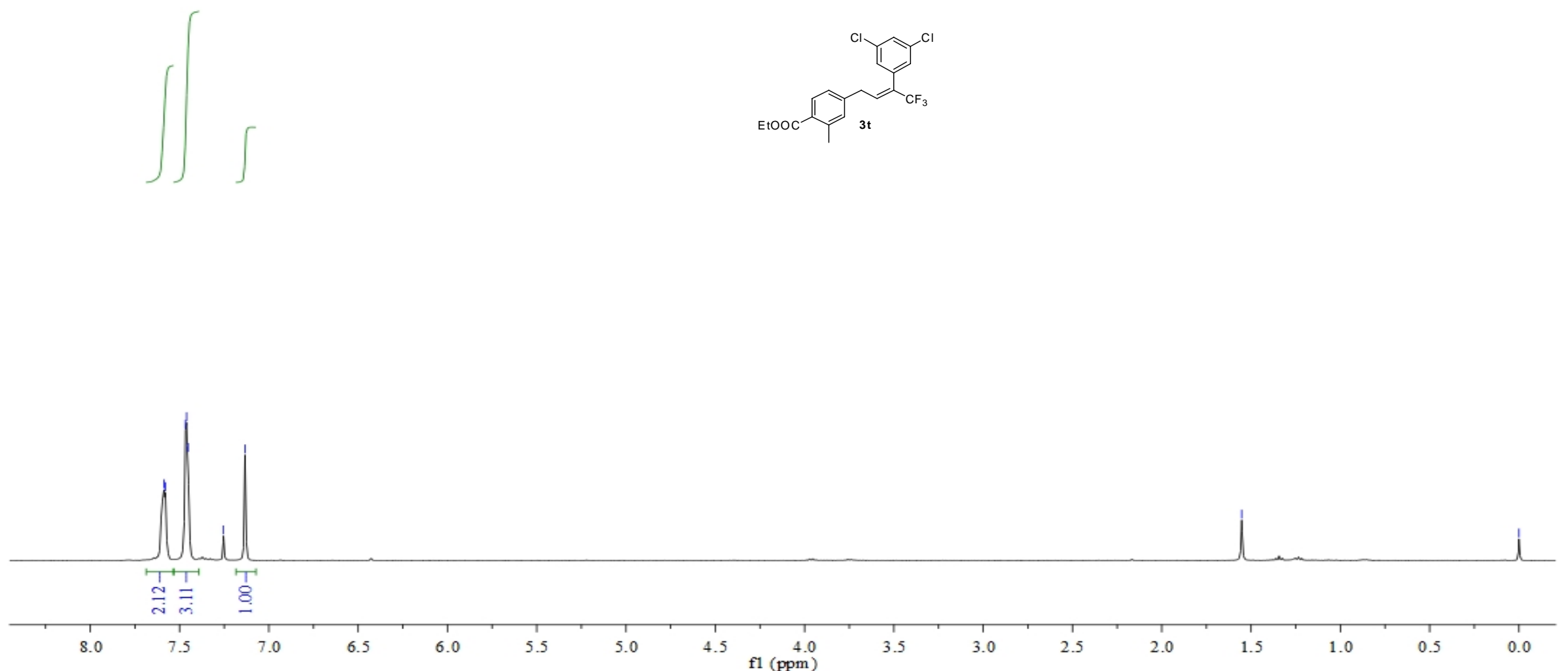


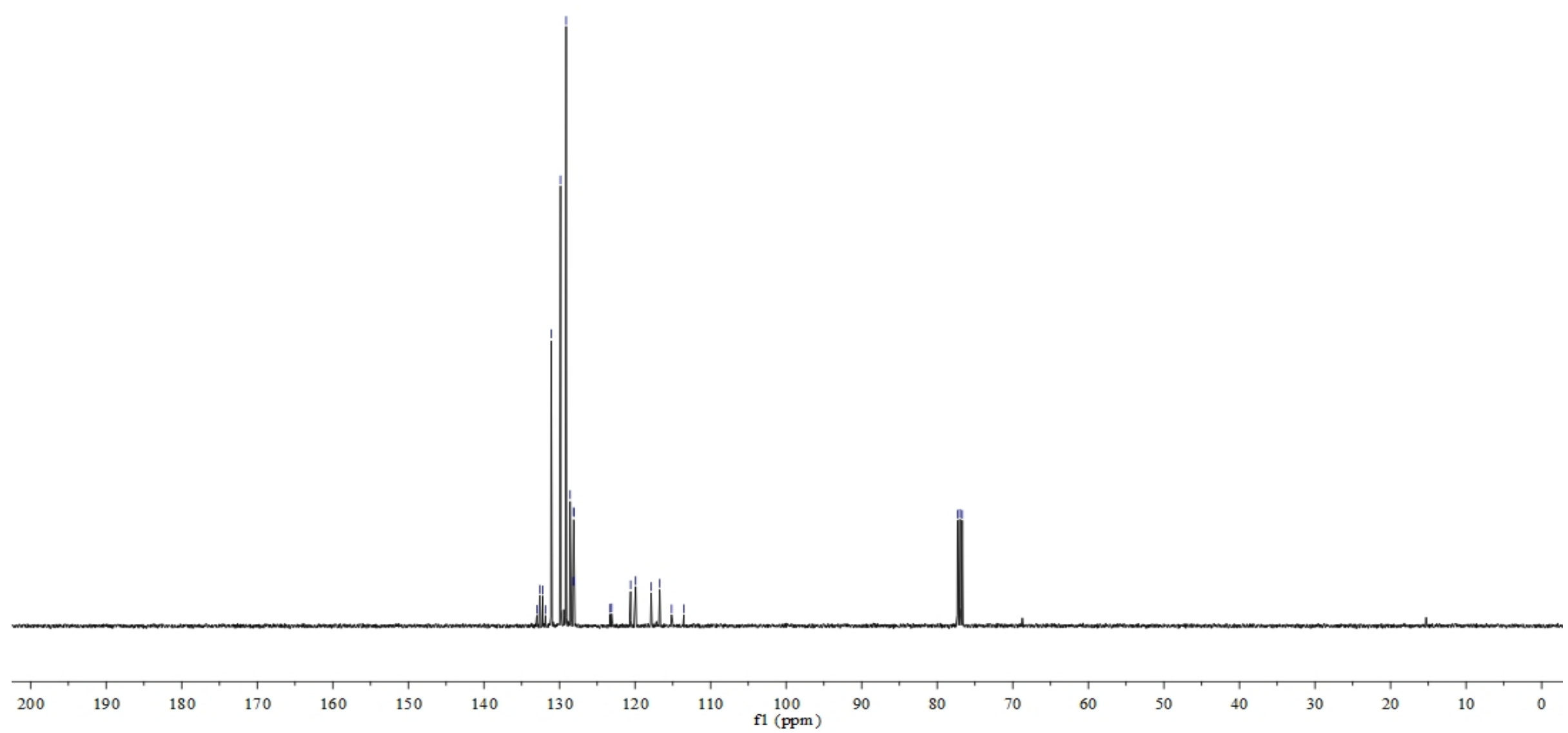


${ }^{19}$ F-NMR
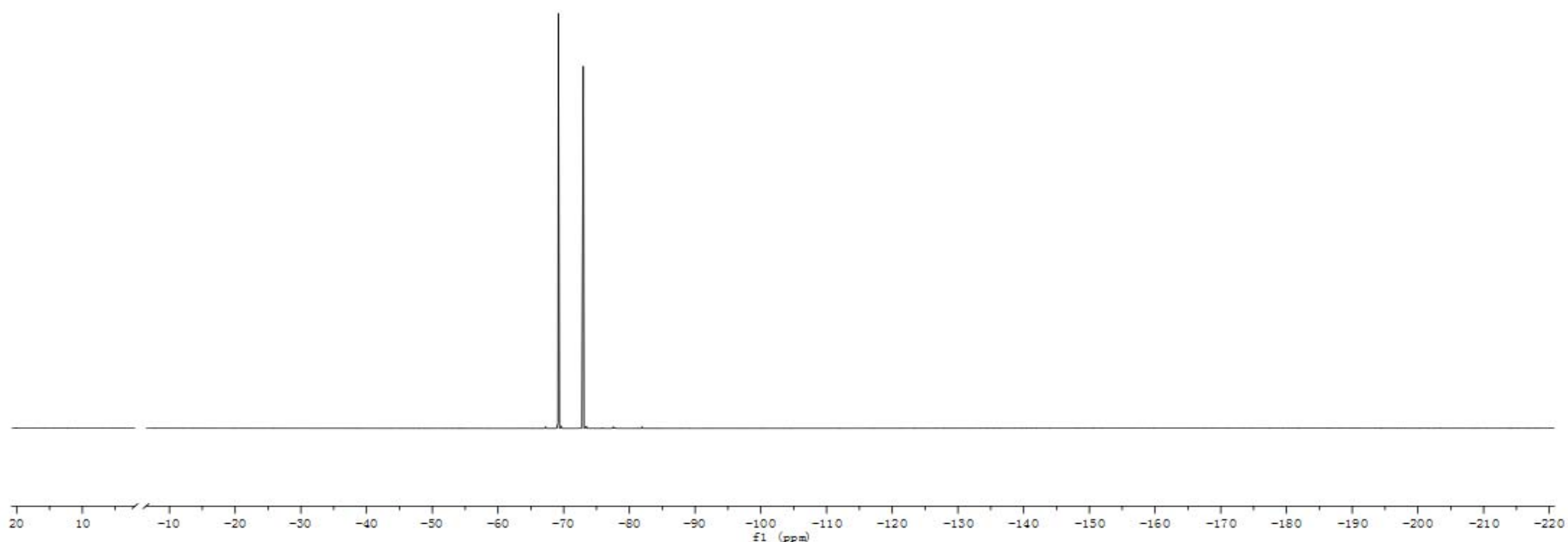


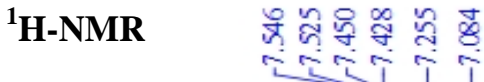
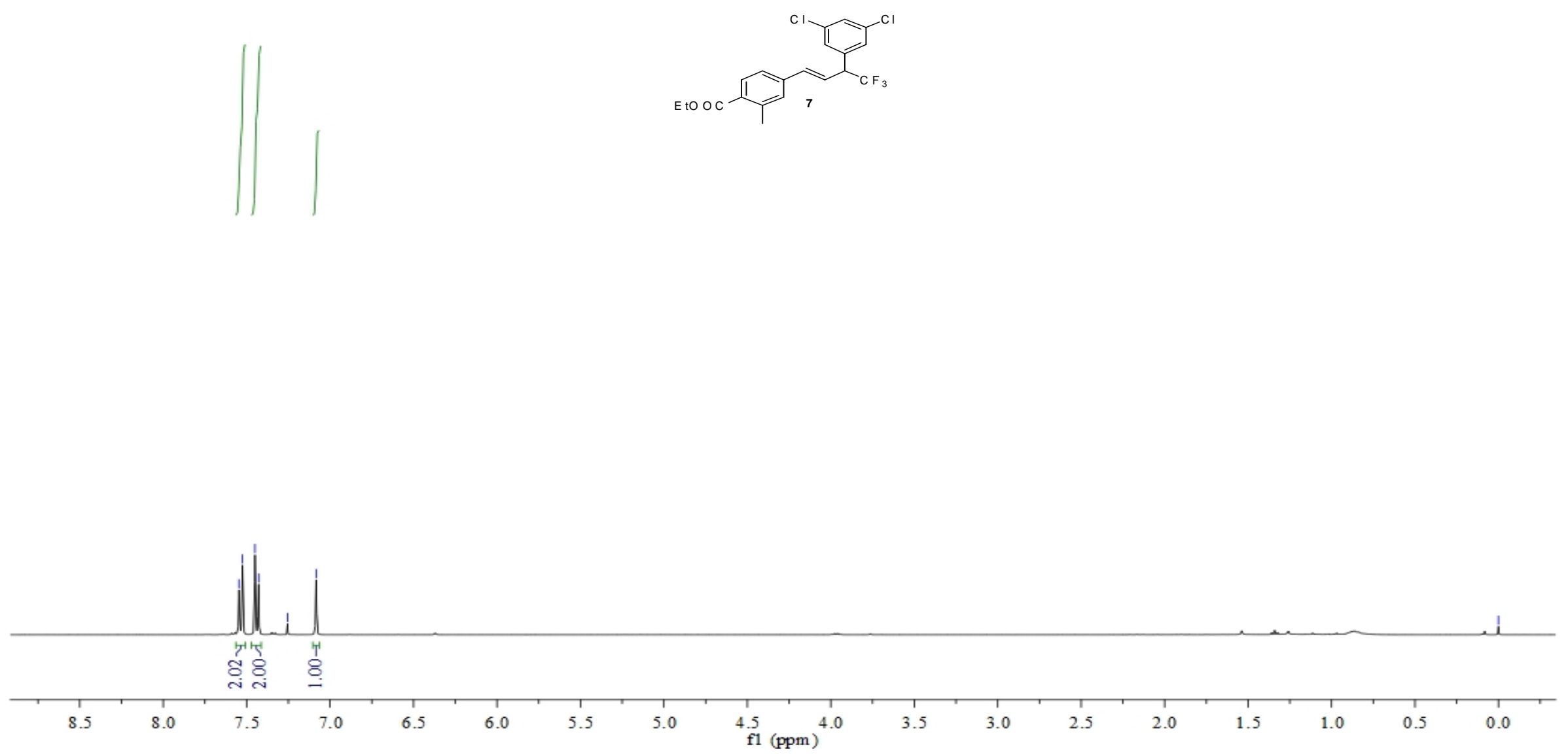
${ }^{13}$ C-NMR

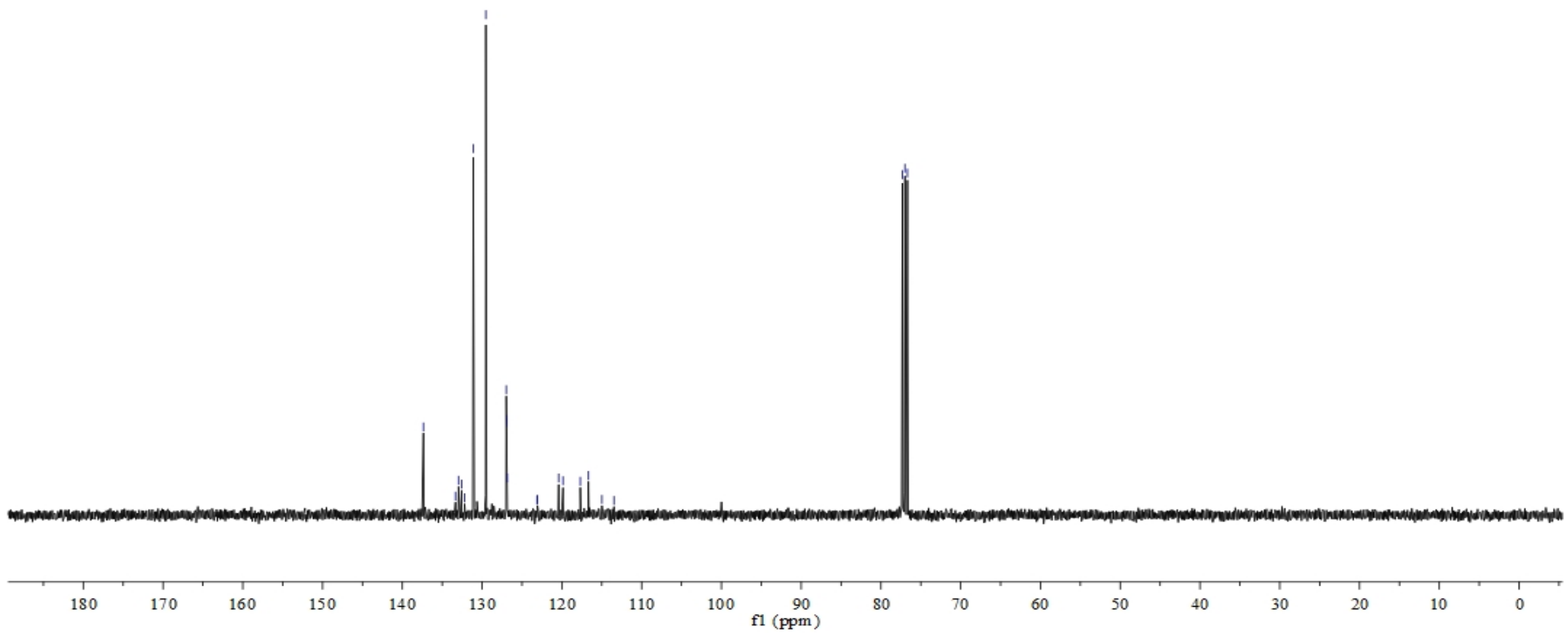


${ }^{19}$ F-NMR

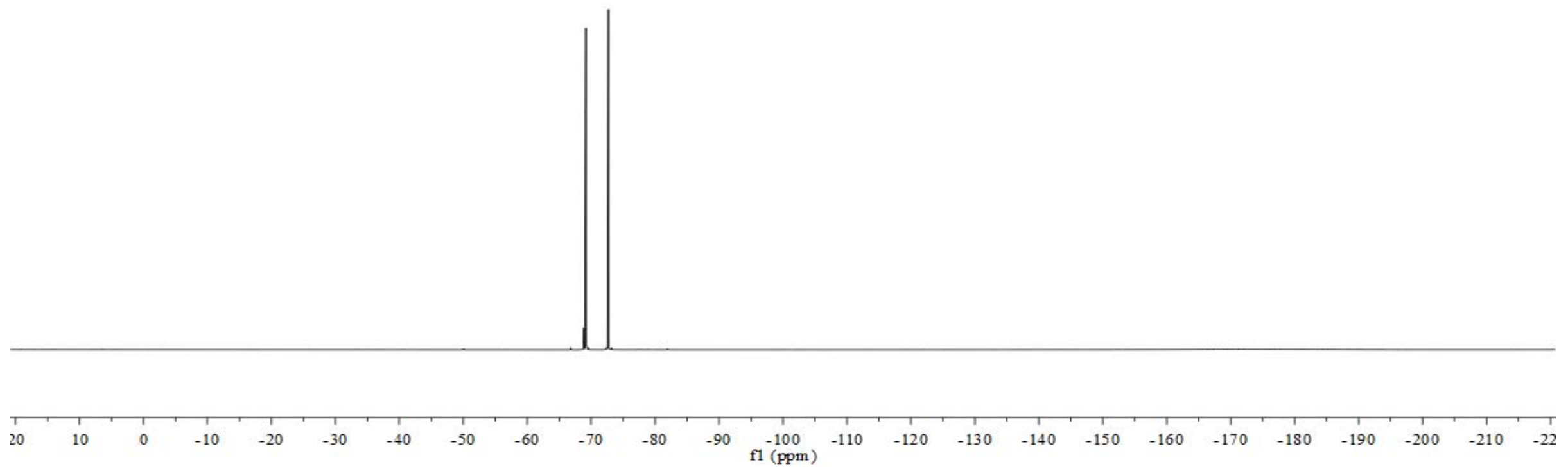



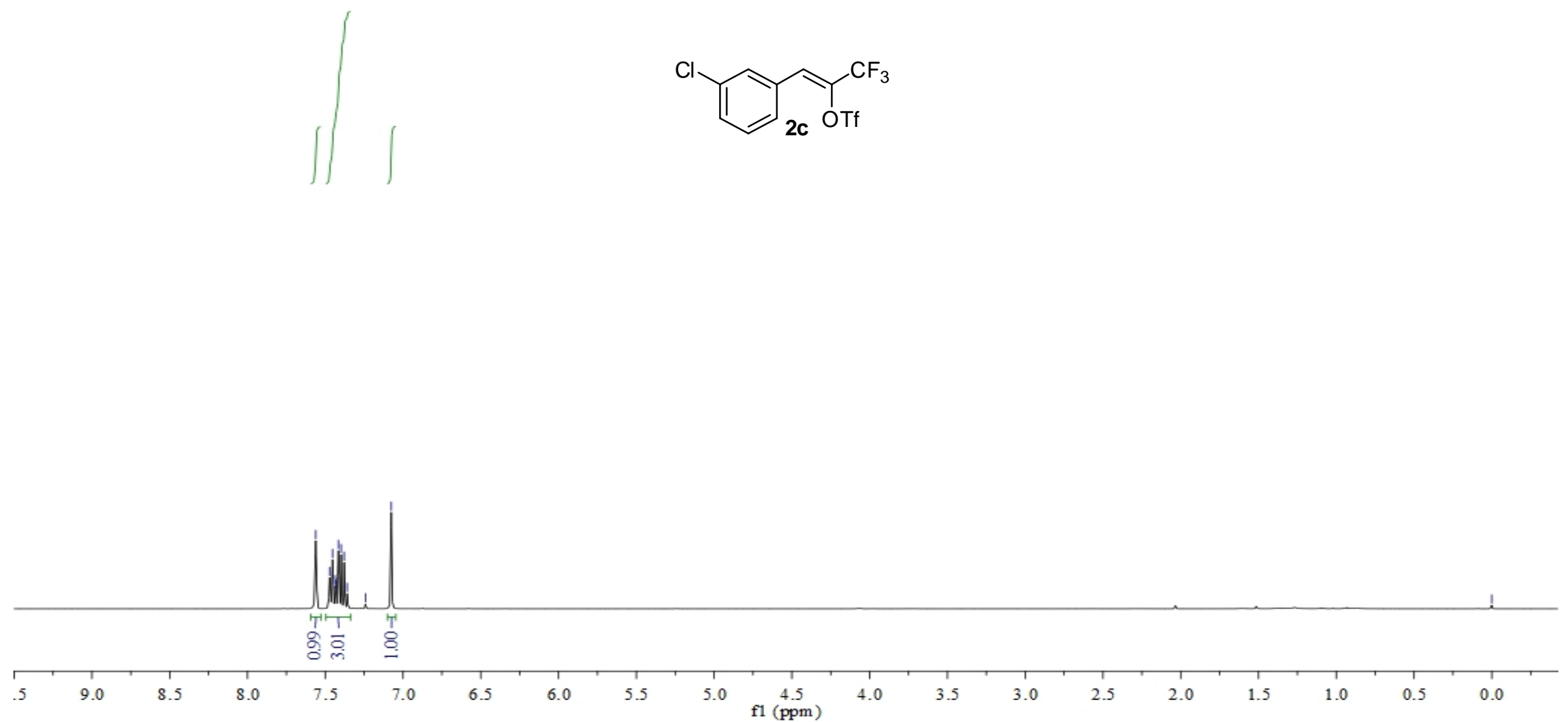


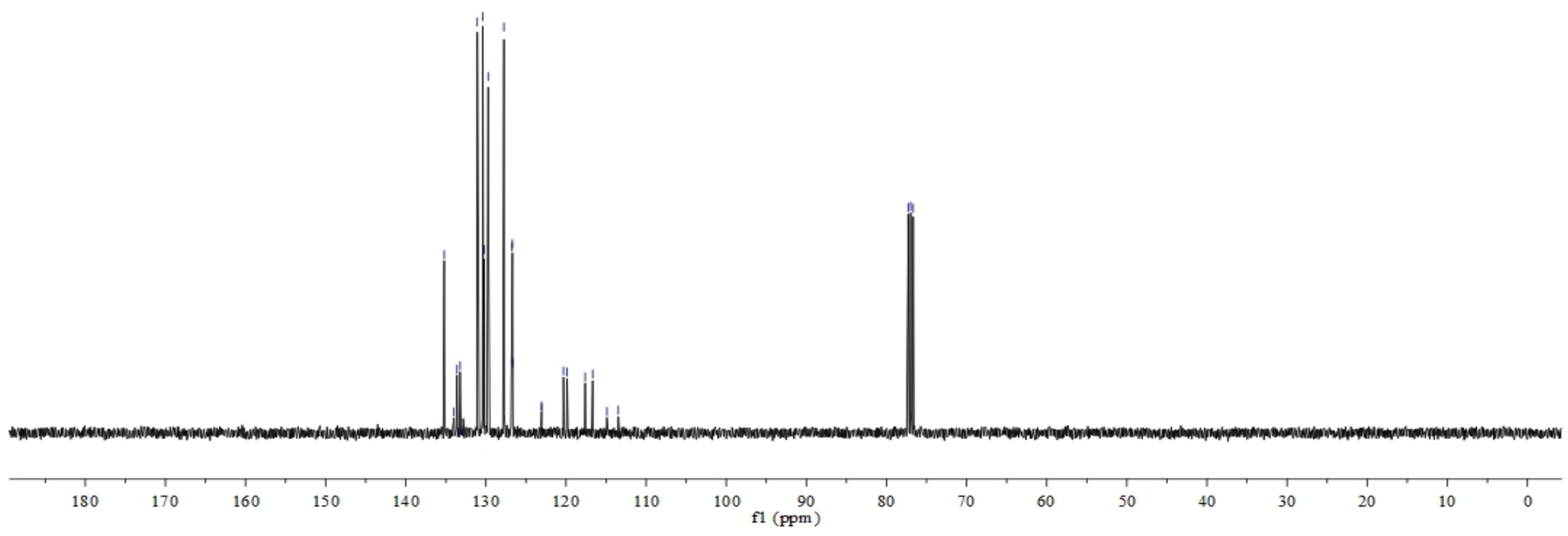


${ }^{19}$ F-NMR

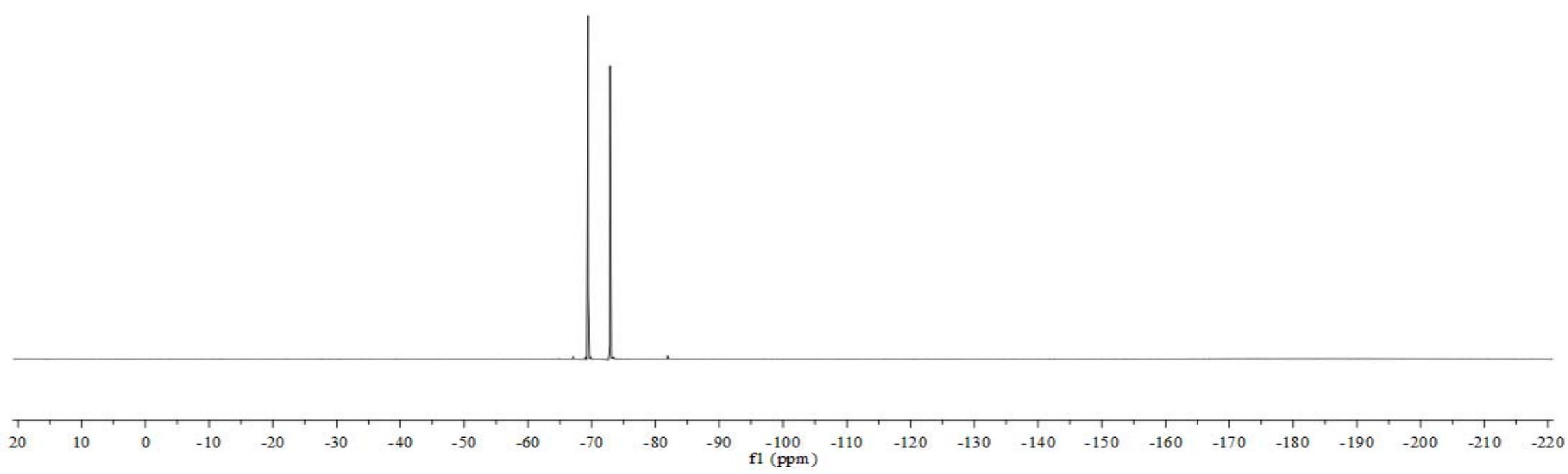




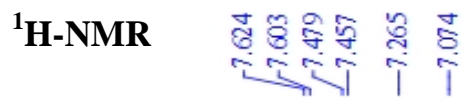
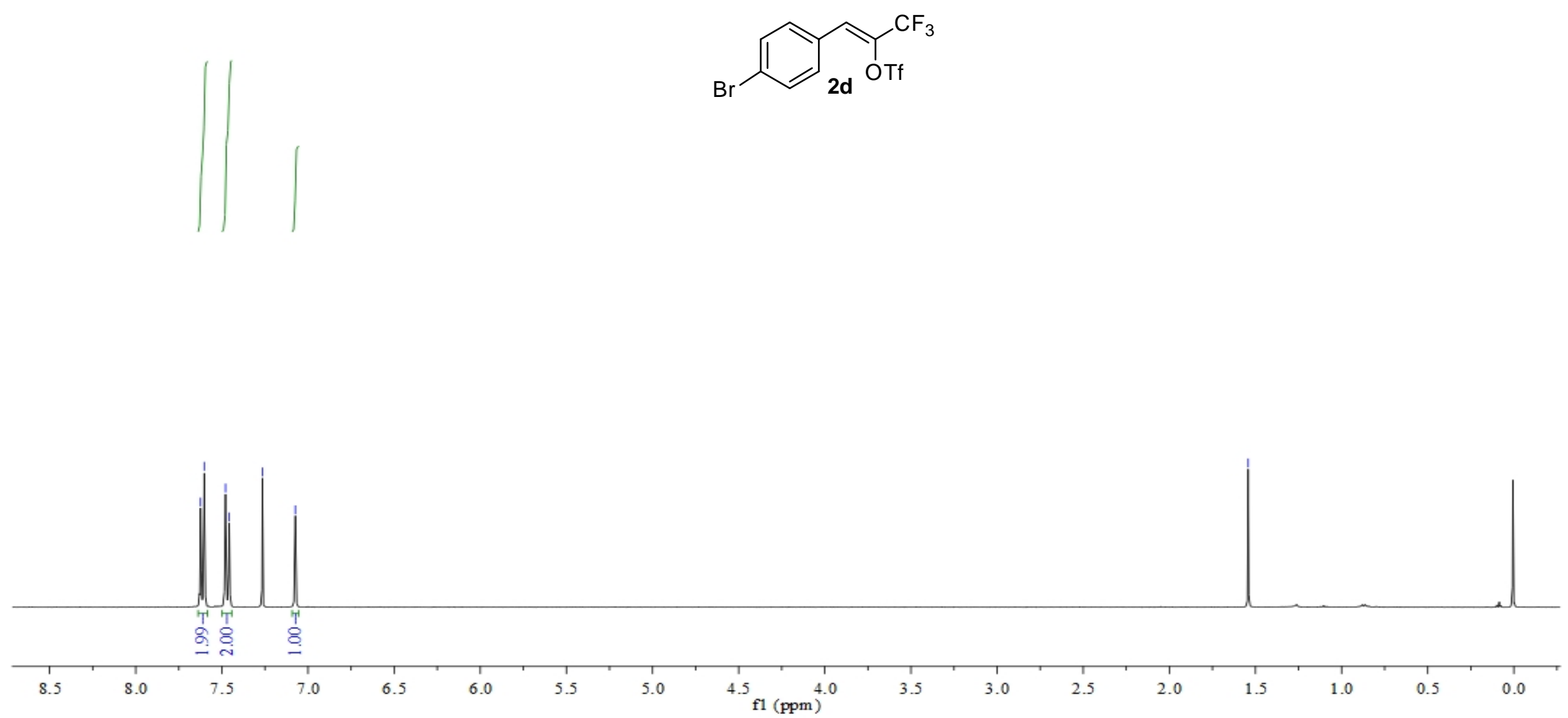
${ }^{13}$ C-NMR

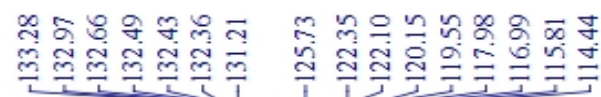

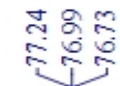

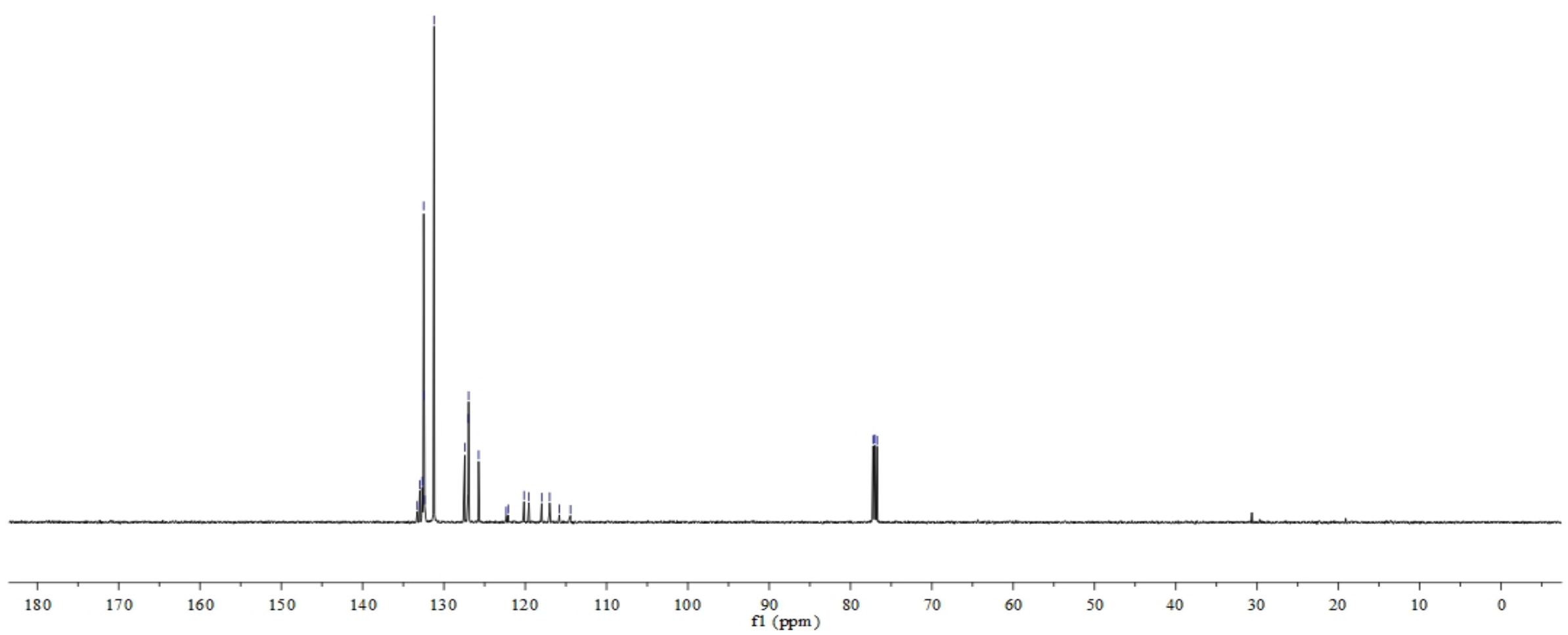


${ }^{19}$ F-NMR

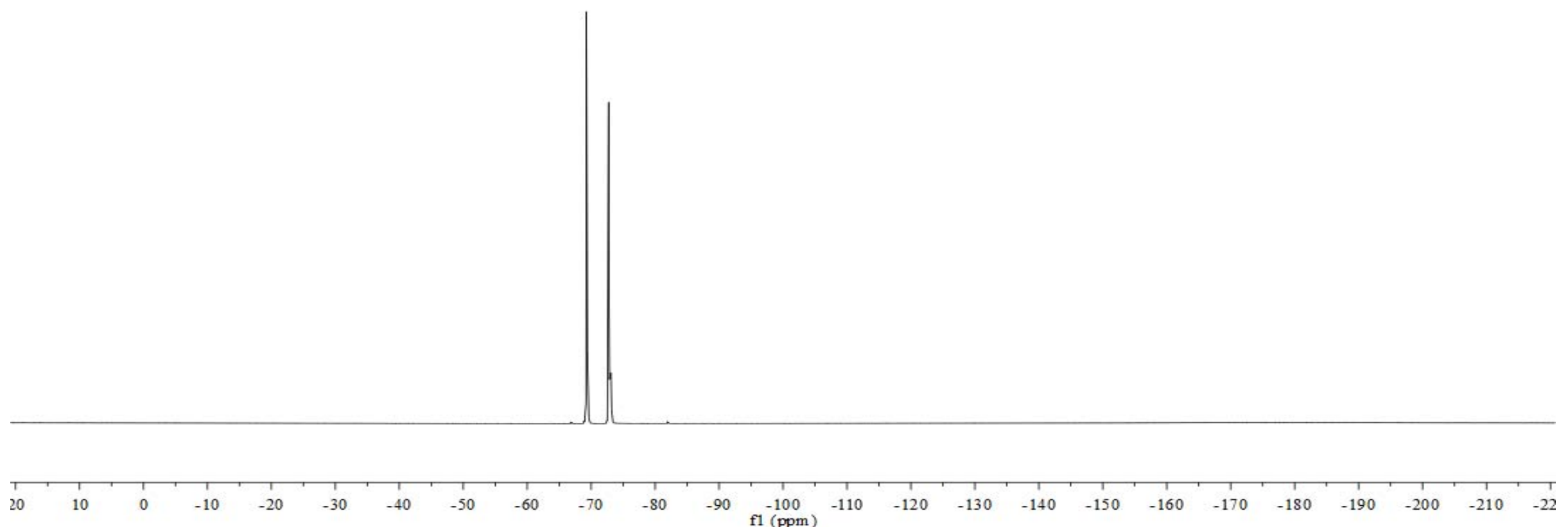


${ }^{1}$ H-NMR
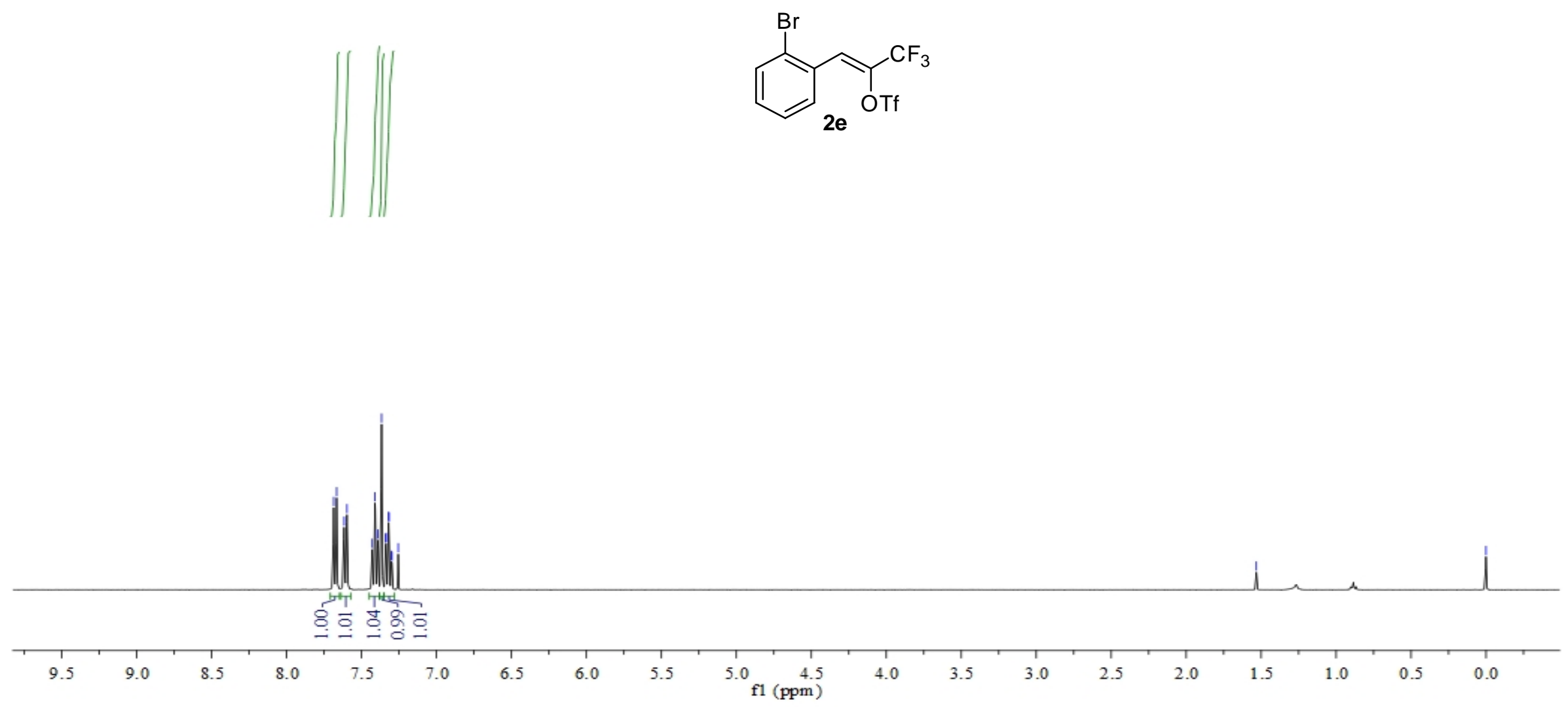


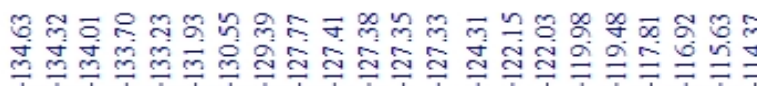

${ }^{13}$ C-NMR

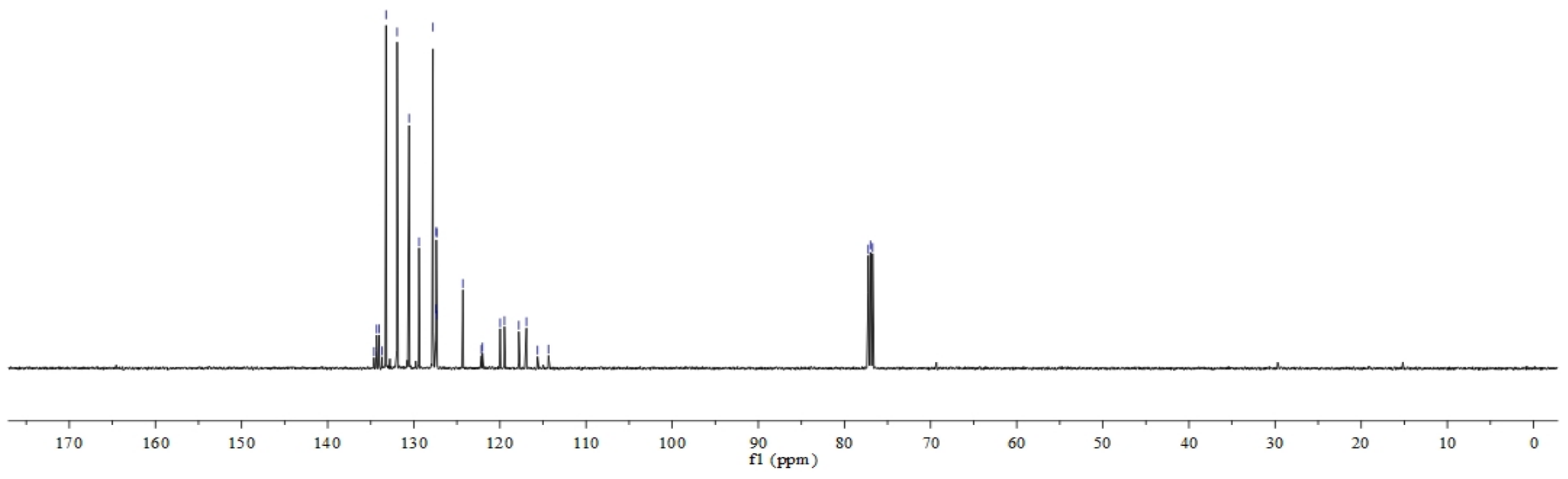


${ }^{19}$ F-NMR

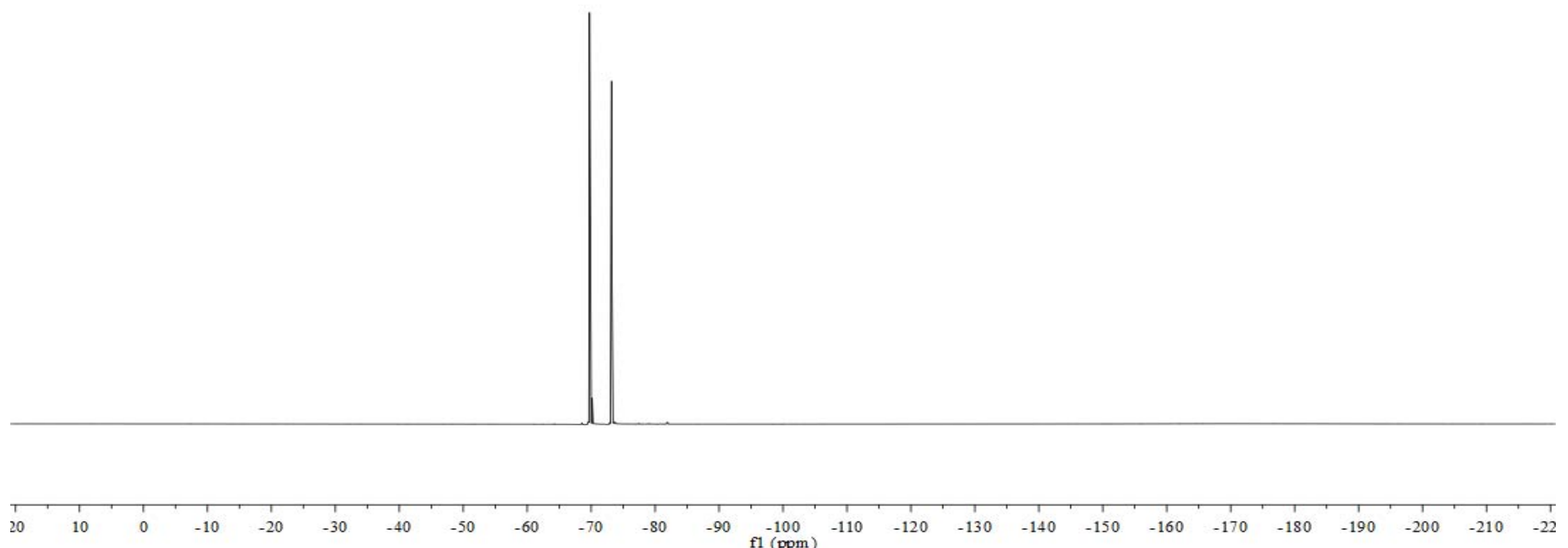


${ }^{1}$ H-NMR

ज䨌

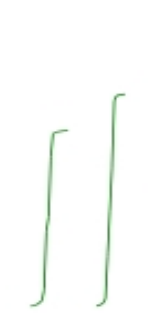

के กี

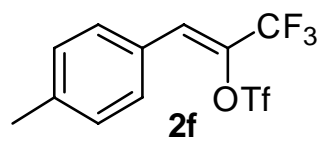

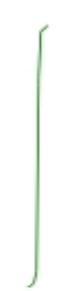

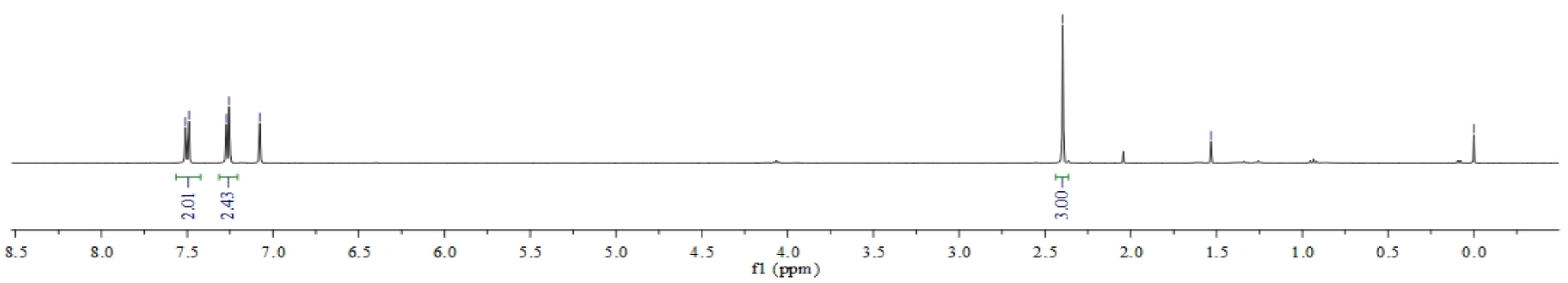


${ }^{13}$ C-NMR

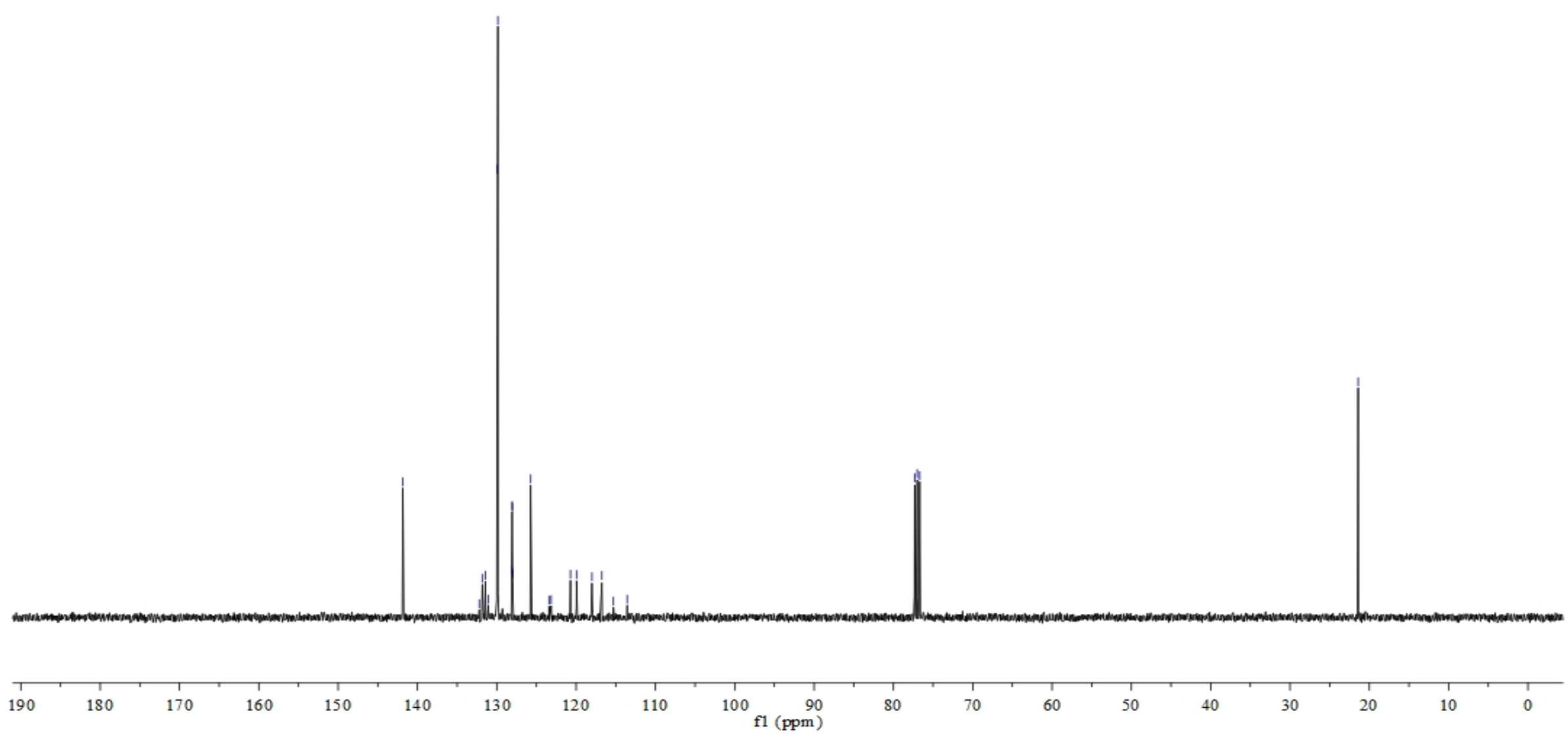


${ }^{19}$ F-NMR
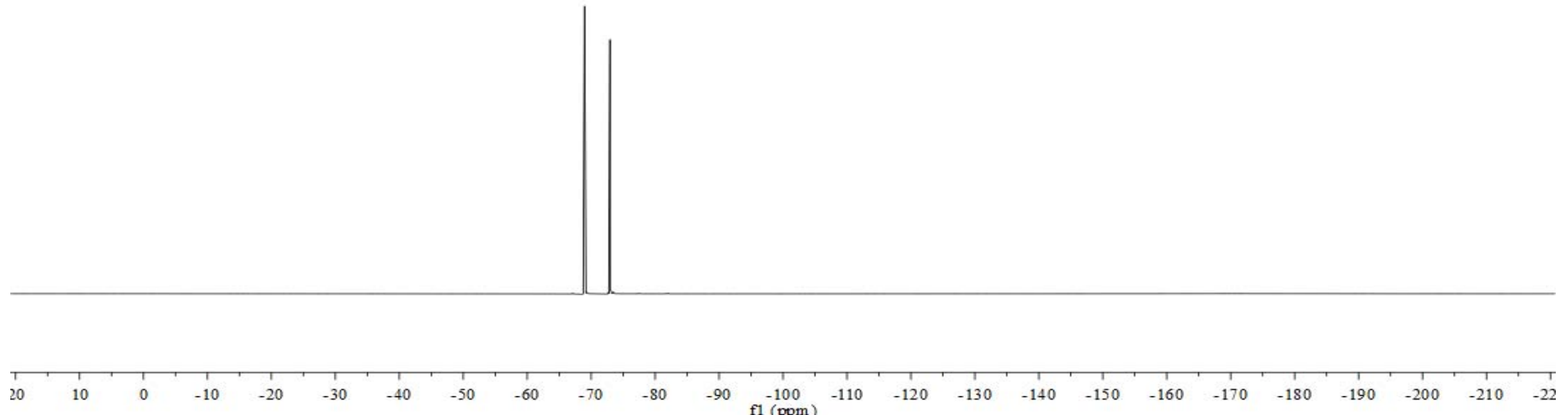
${ }^{1}$ H-NMR के

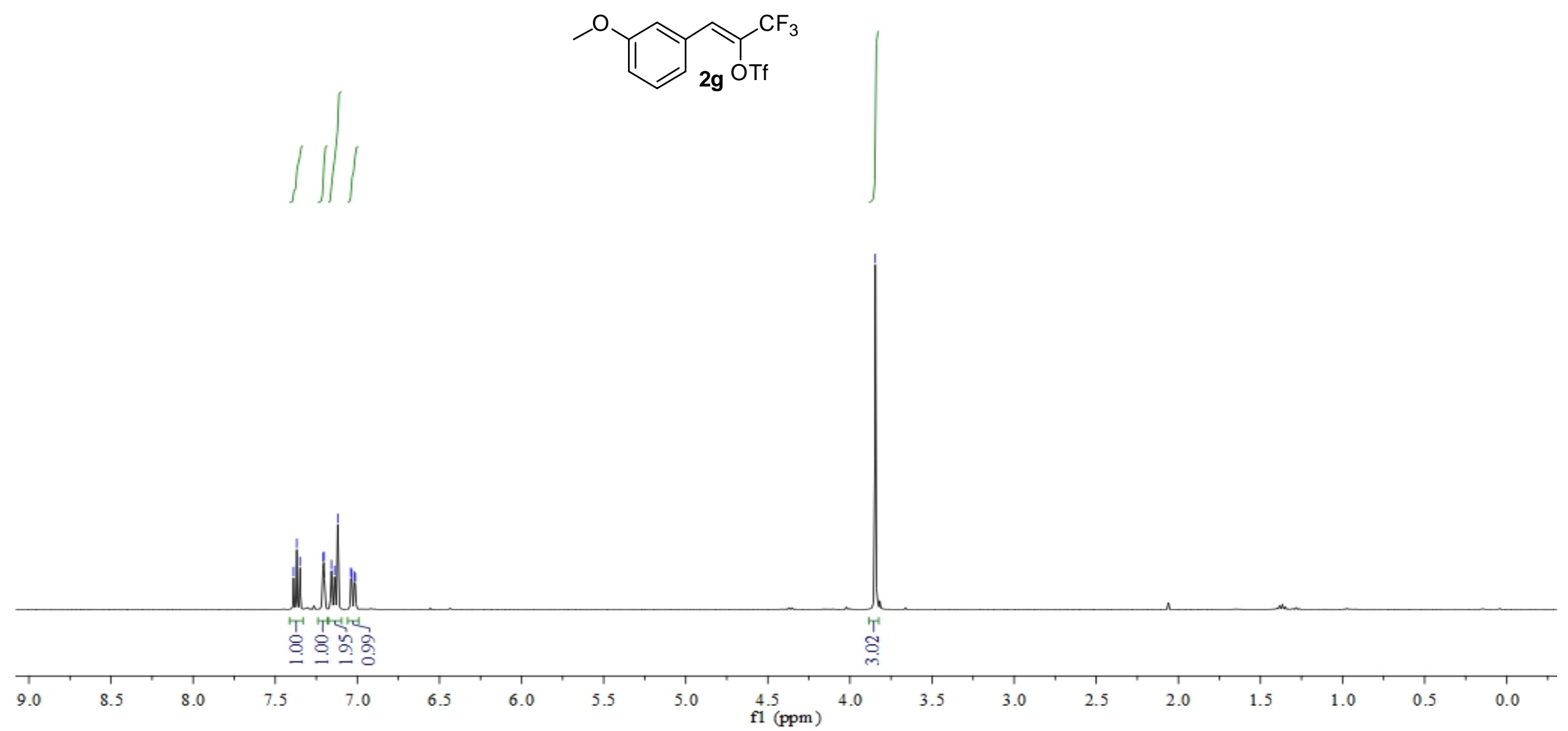




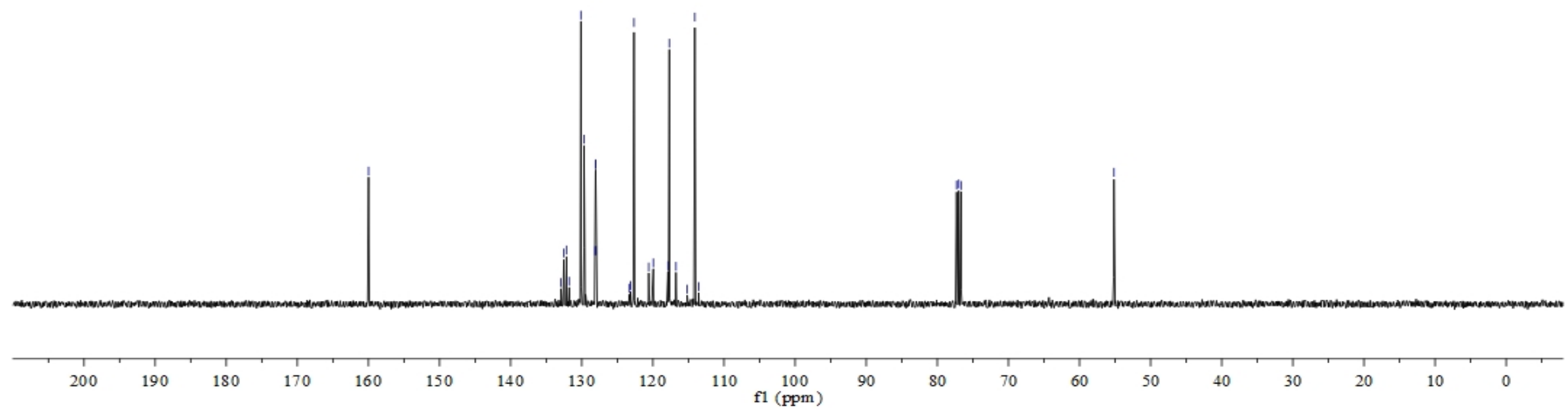


${ }^{19}$ F-NMR
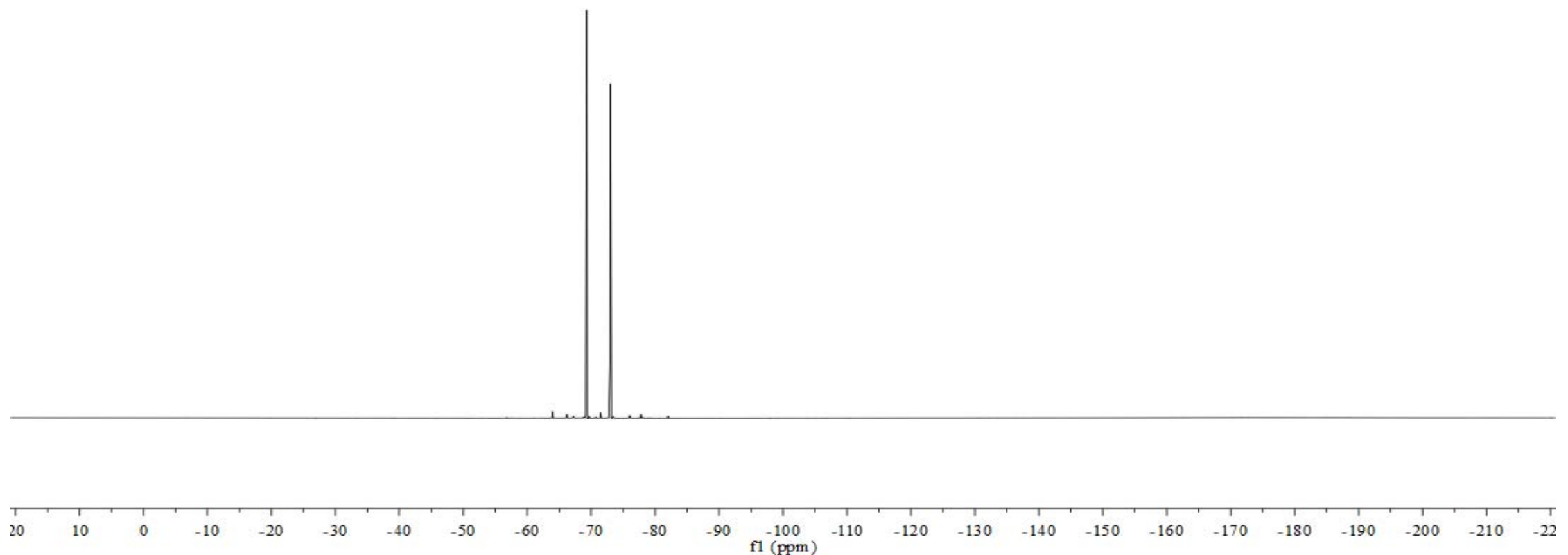


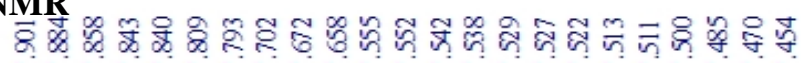

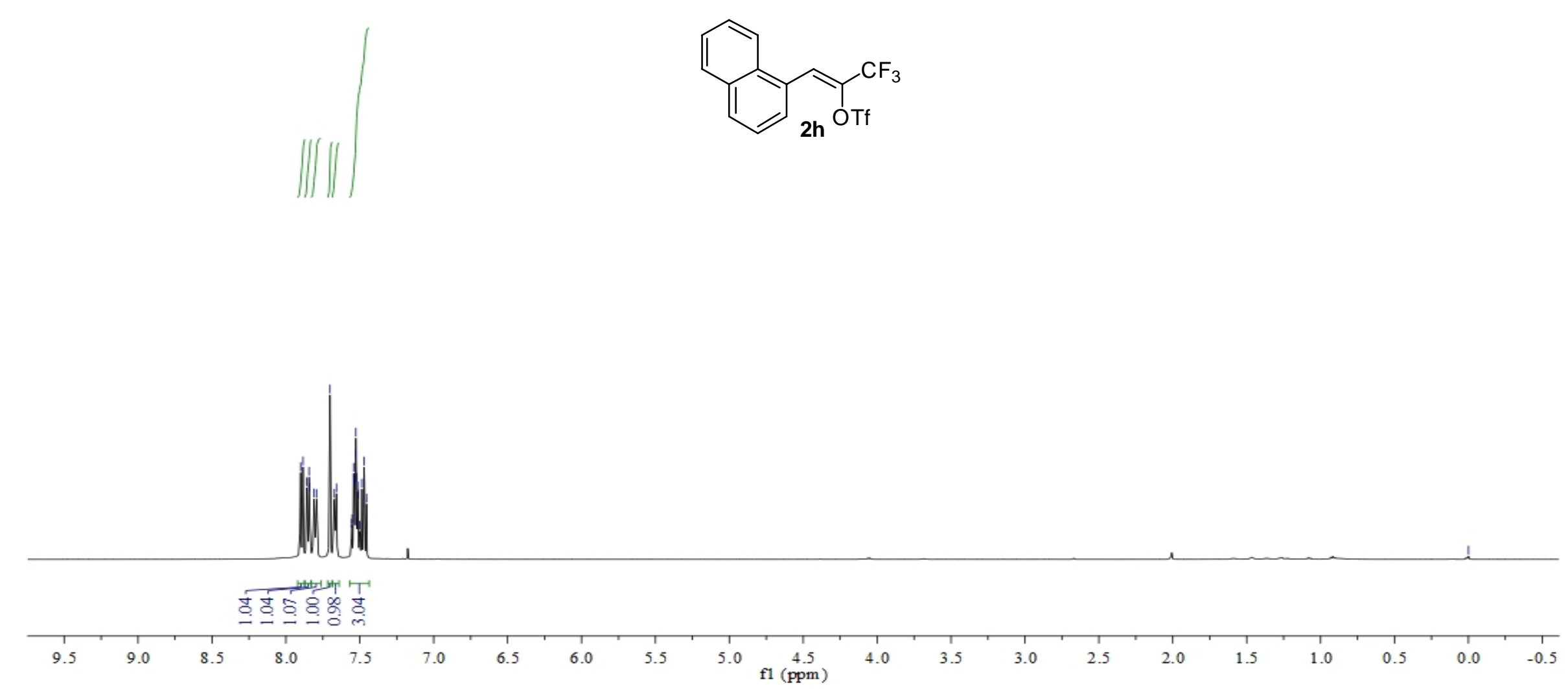


${ }^{13}$ C-NMR

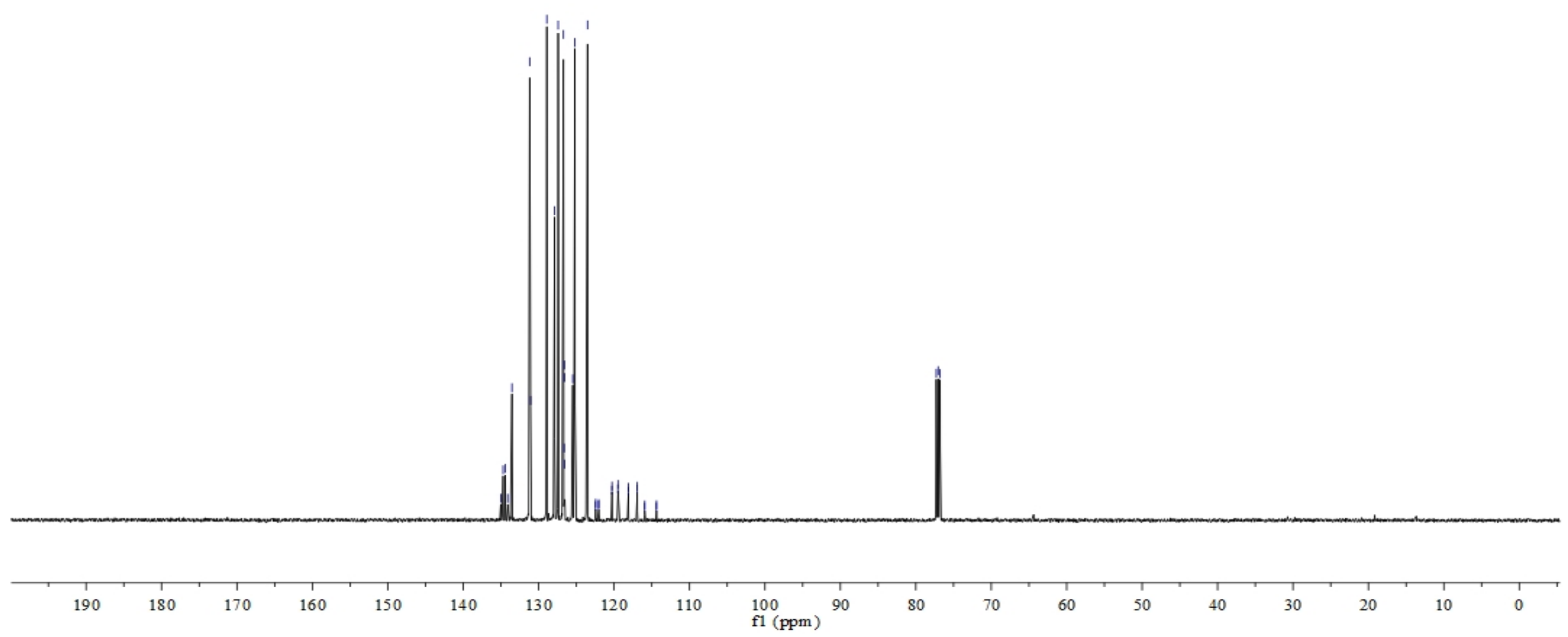


${ }^{19}$ F-NMR
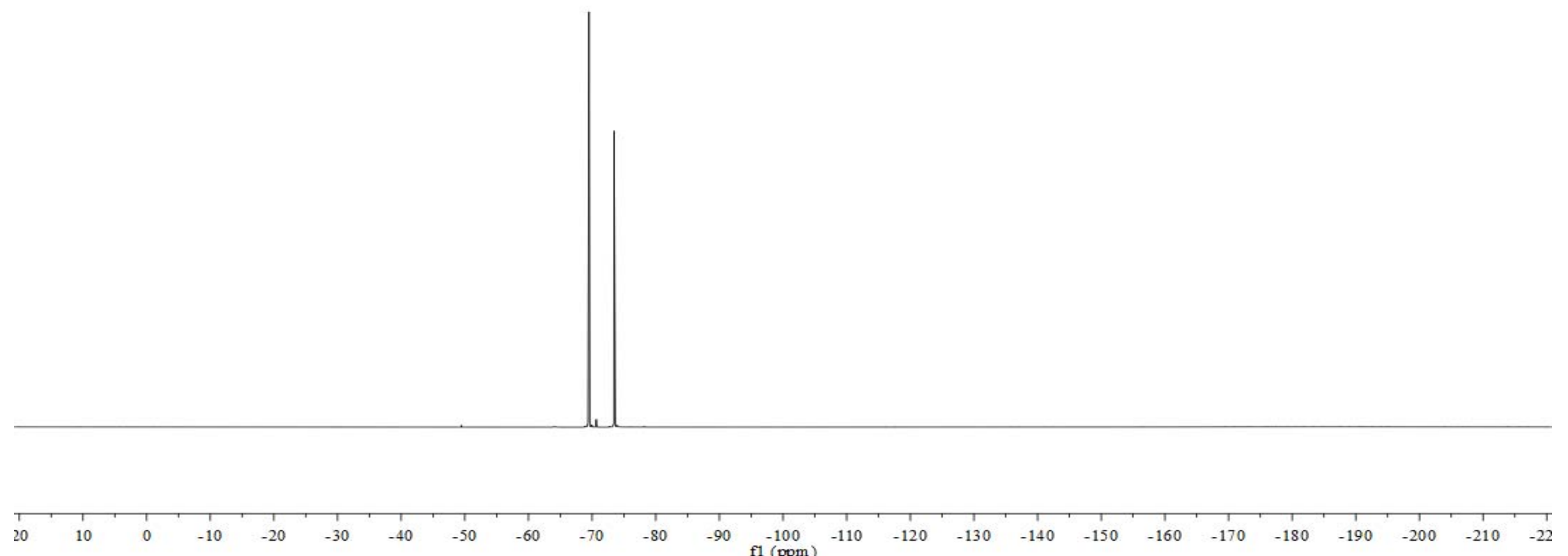
${ }^{1}$ H-NMR
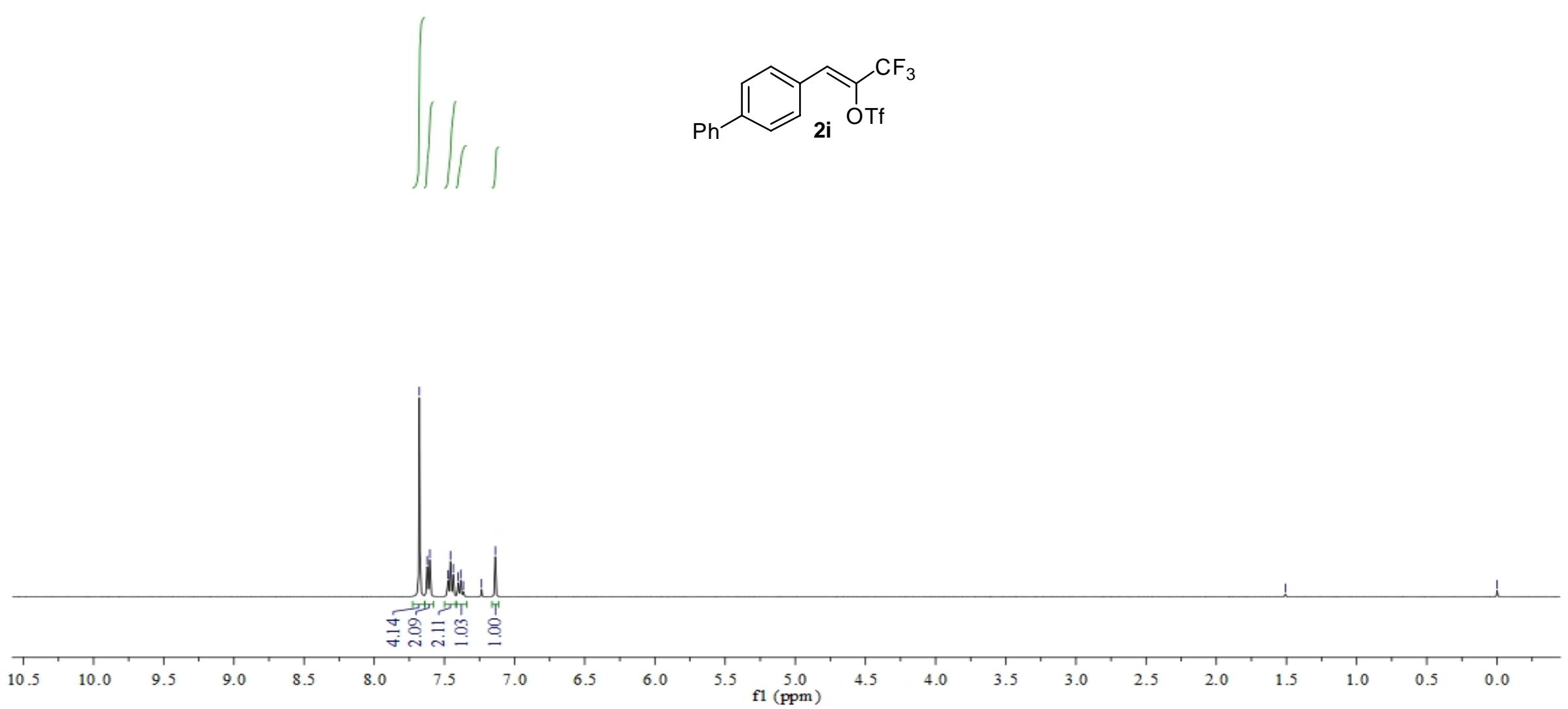


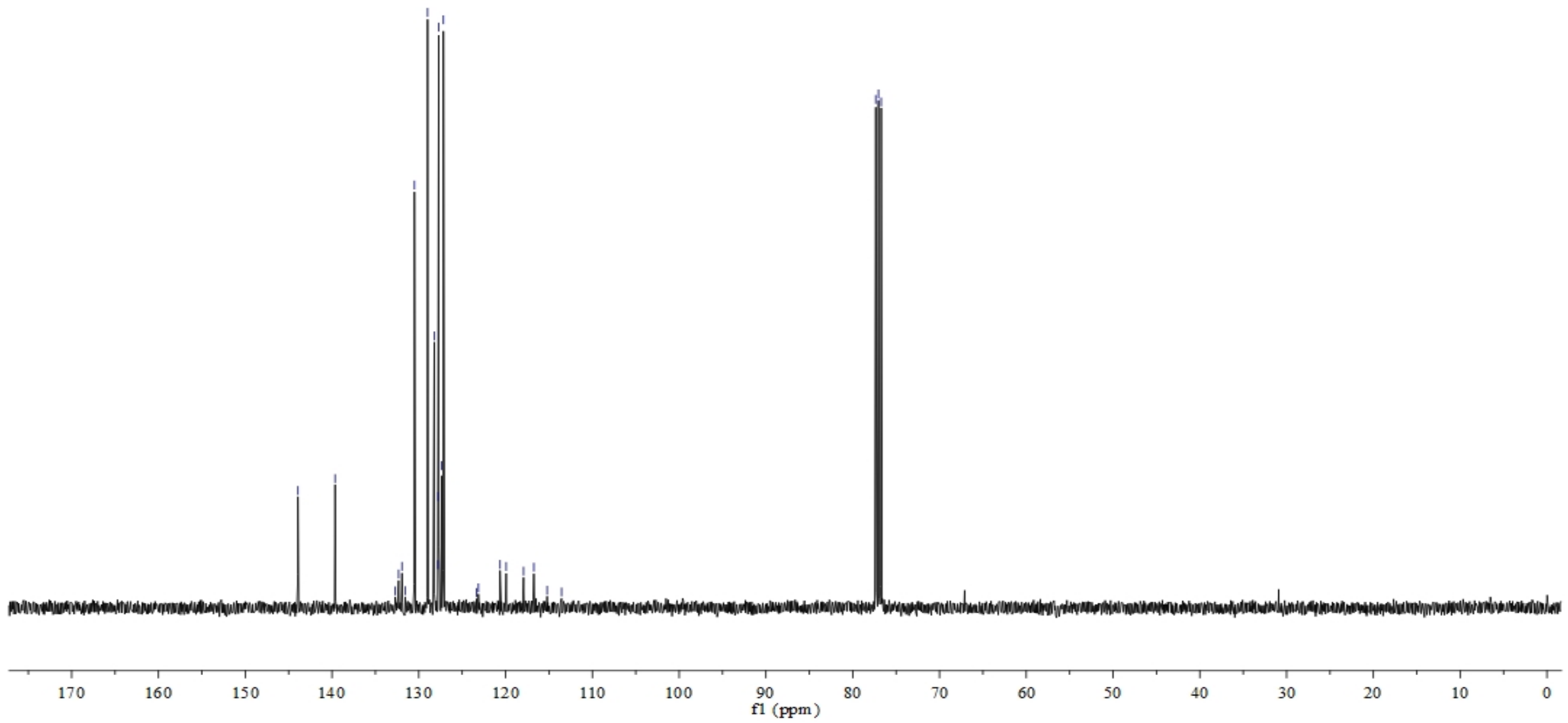


${ }^{19}$ F-NMR

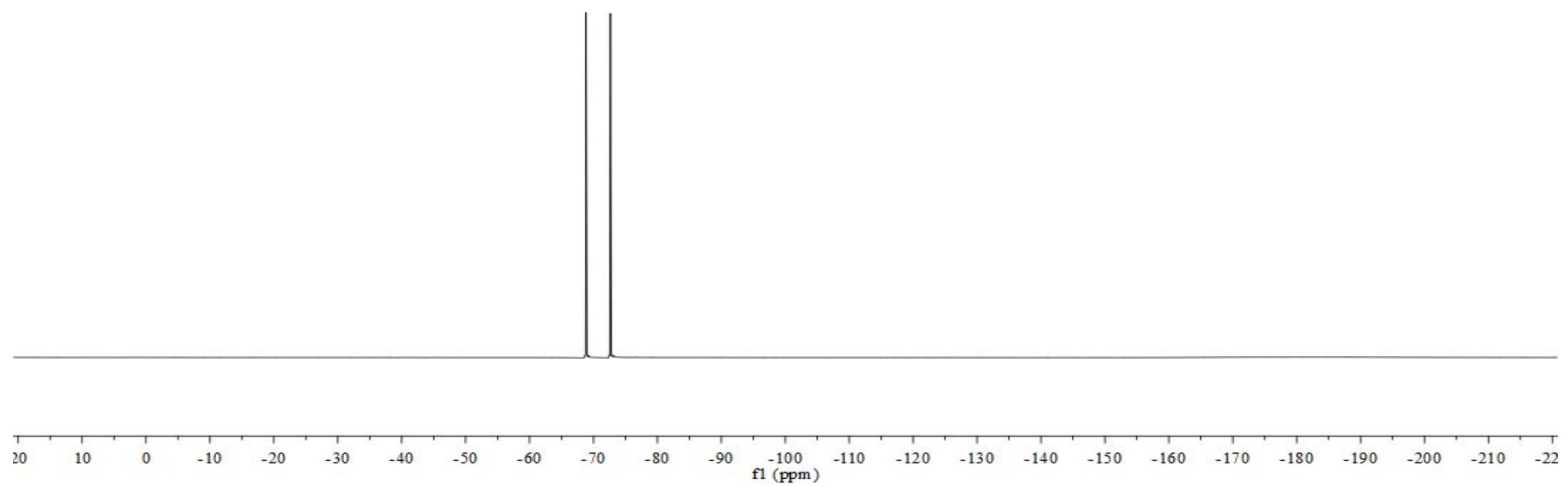




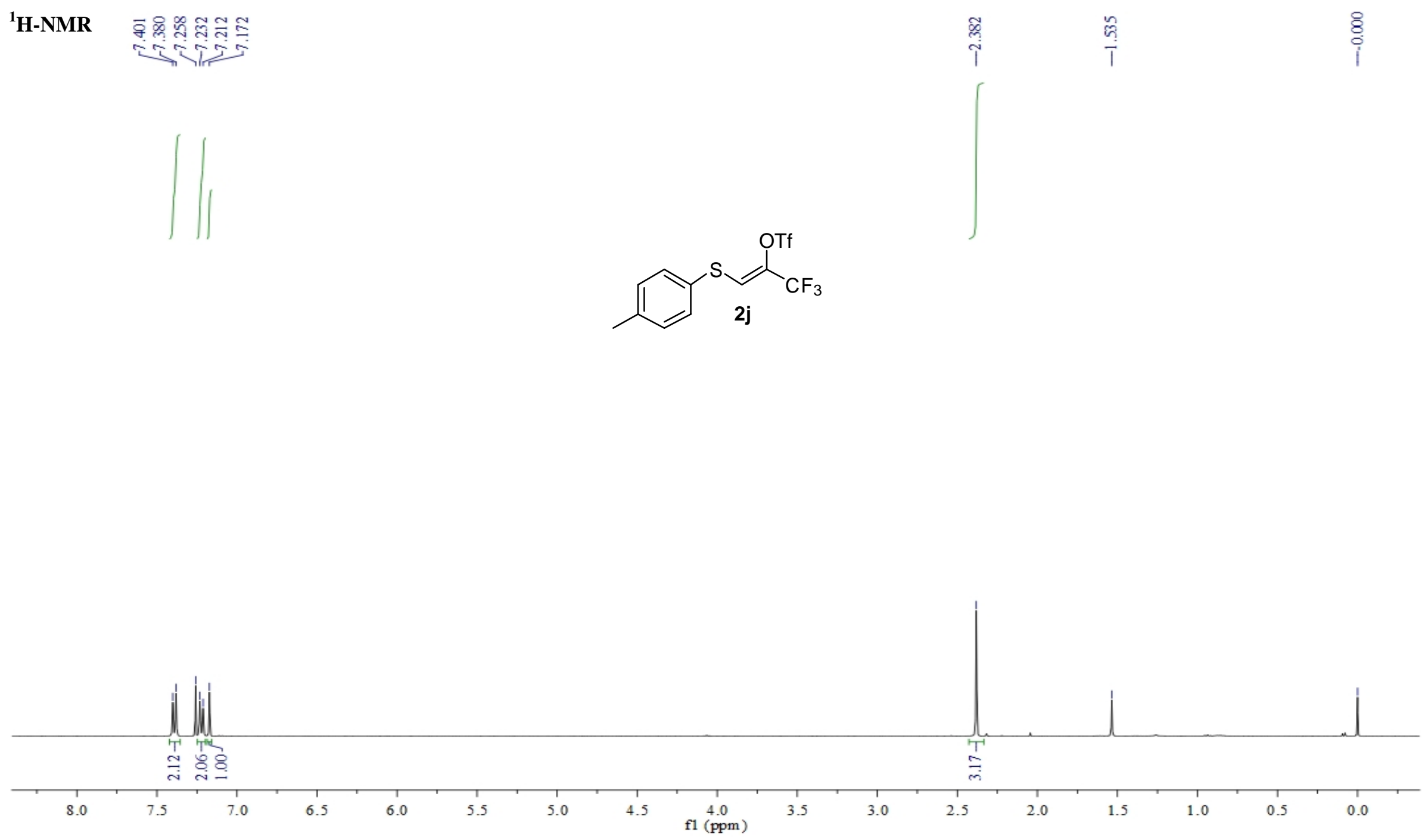


${ }^{13}$ C-NMR

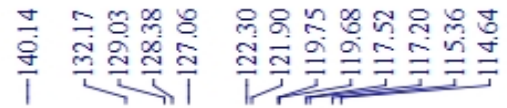

경

$\stackrel{8}{i}$

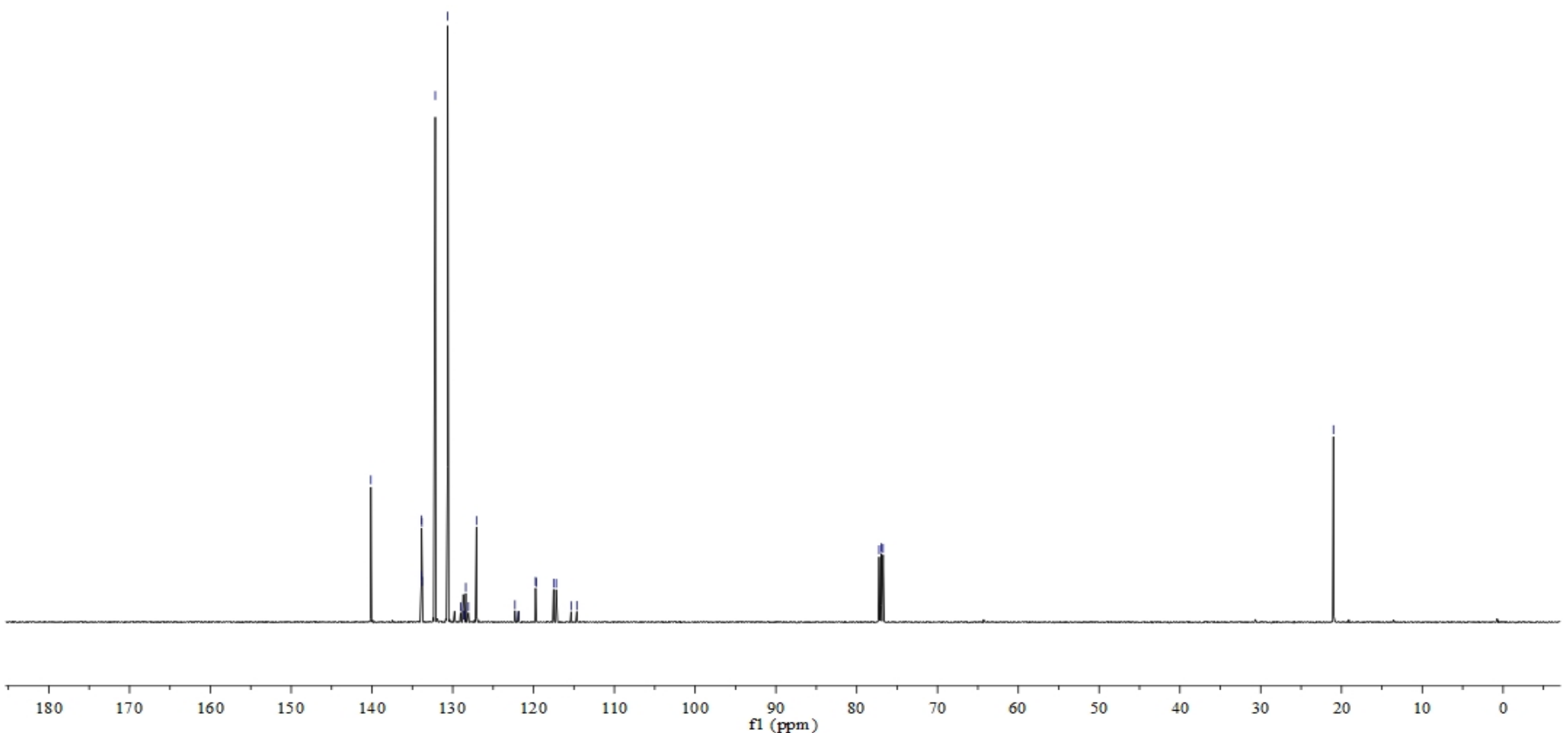


${ }^{19}$ F-NMR

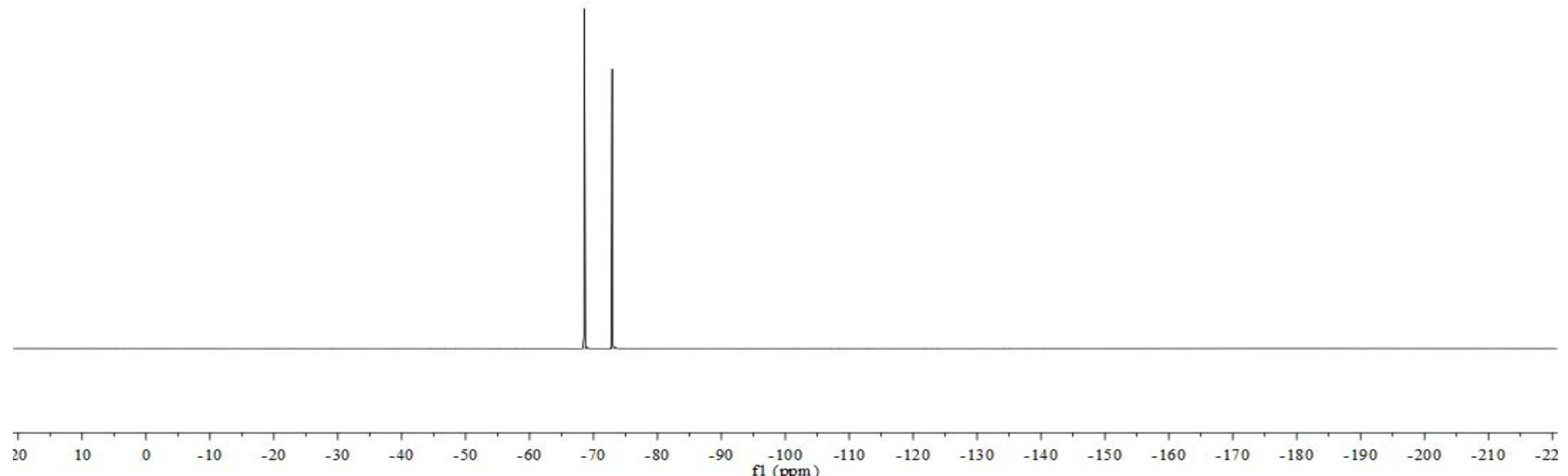



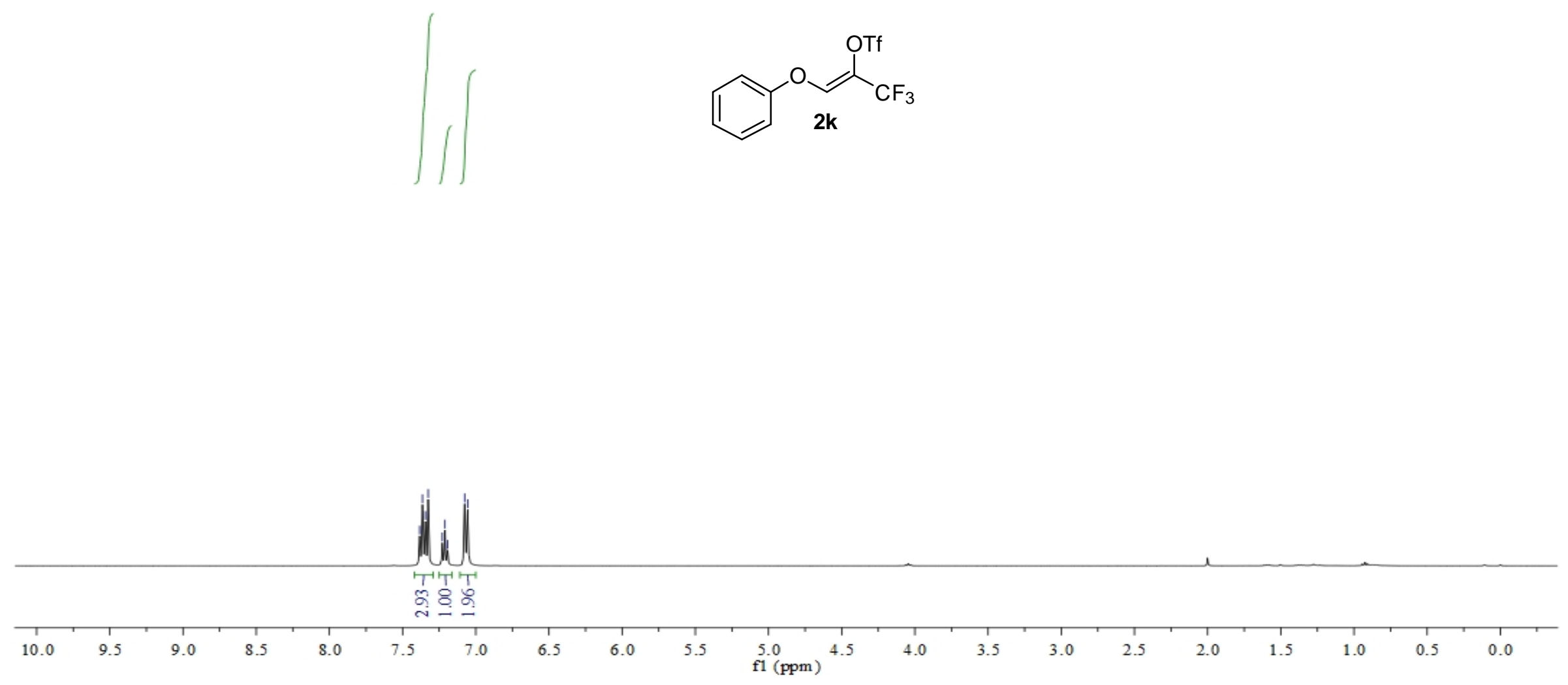


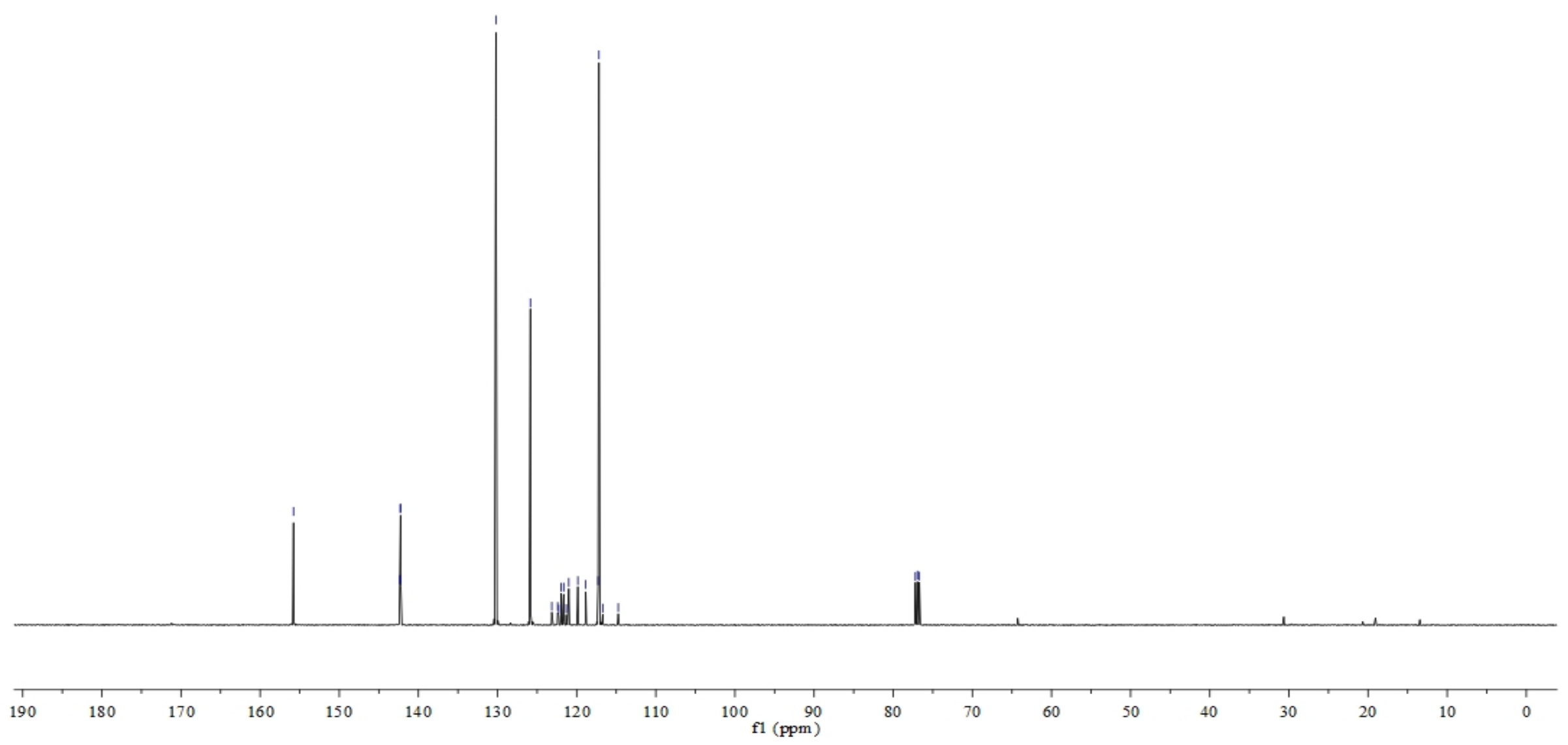


${ }^{19}$ F-NMR

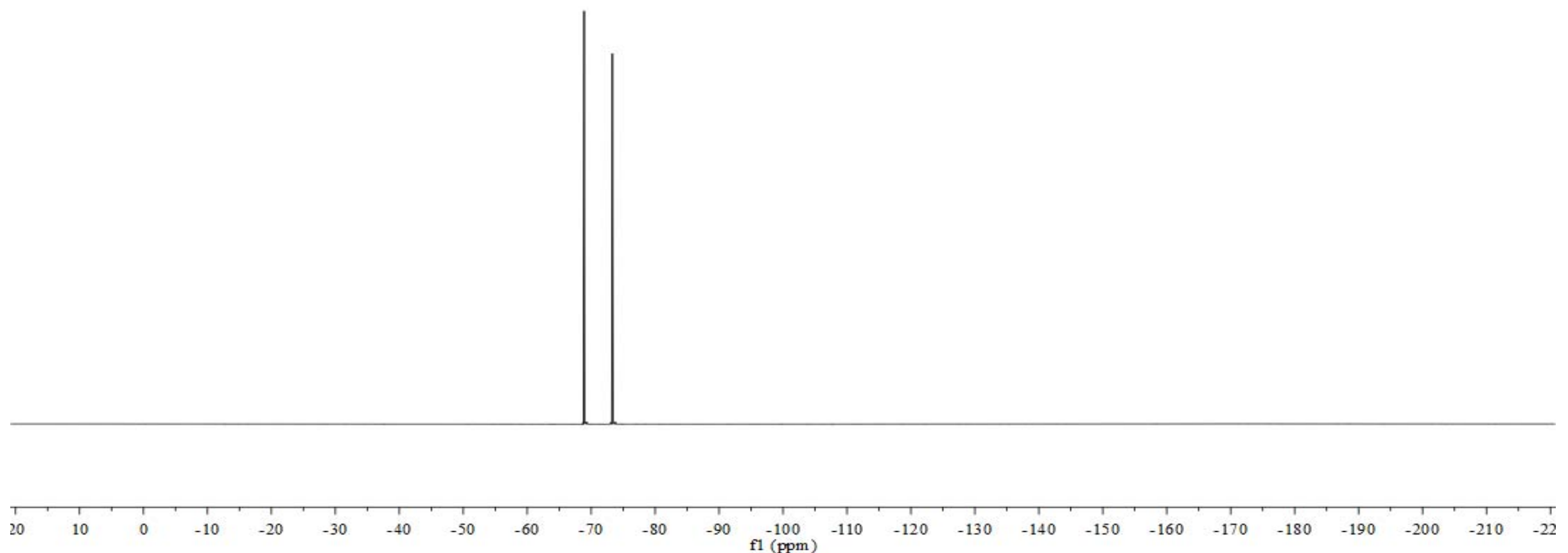


${ }^{1}$ H-NMR
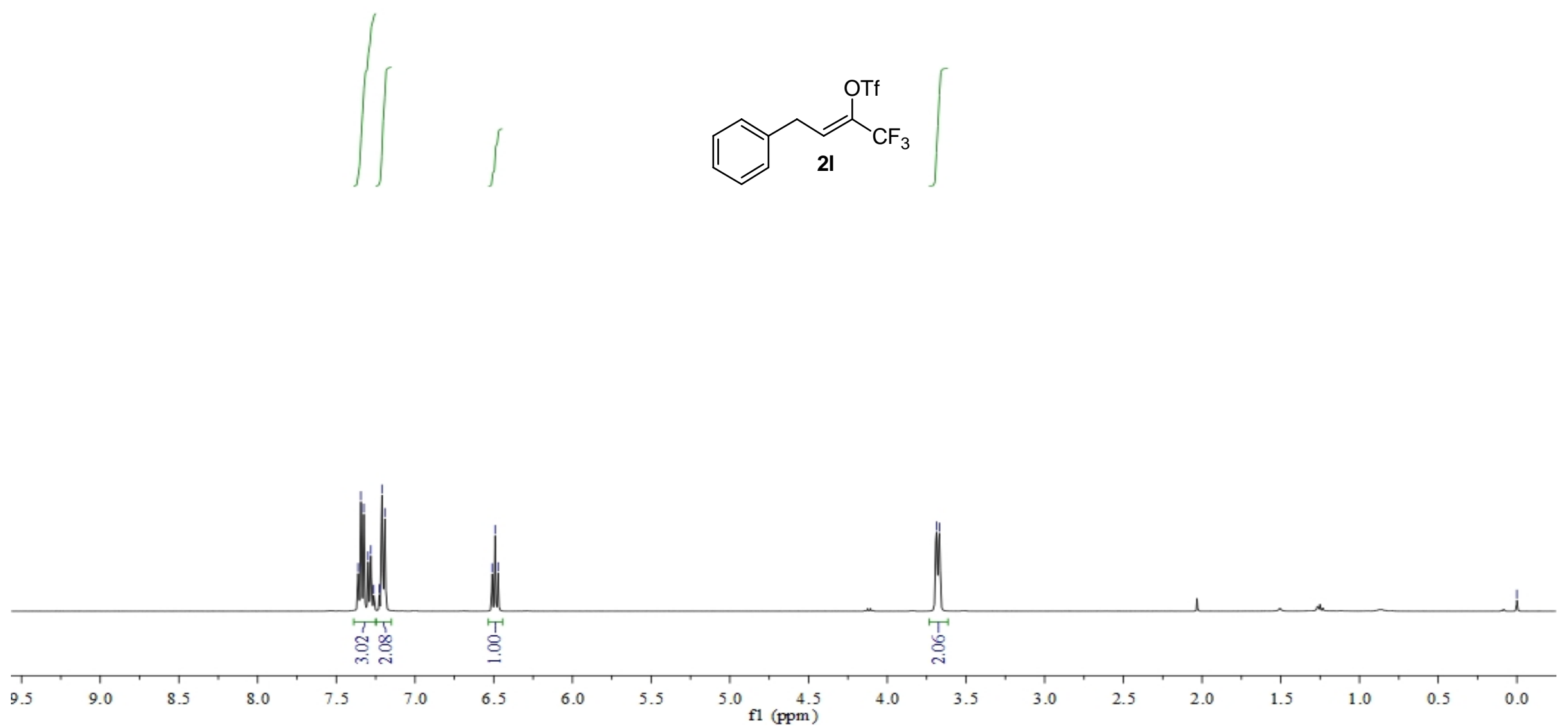


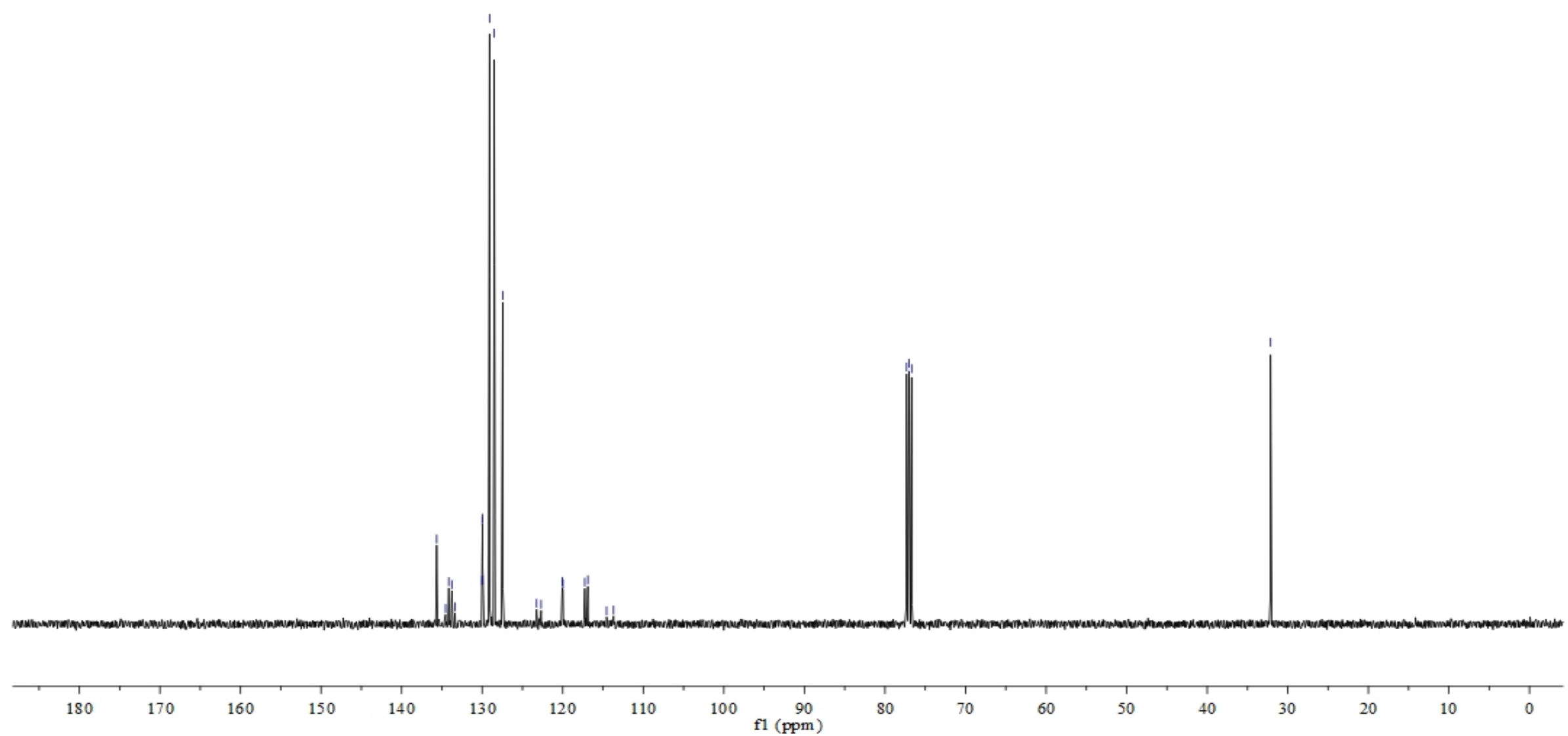


${ }^{19}$ F-NMR

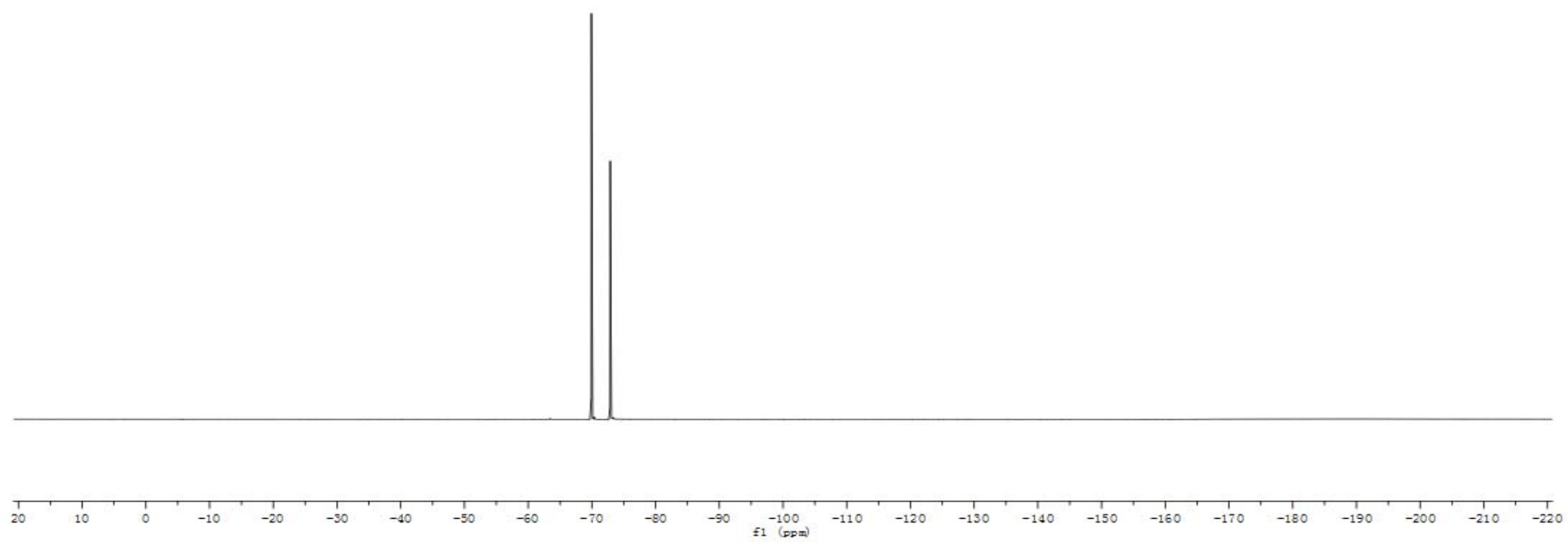




\section{${ }^{1}$ H-NMR}

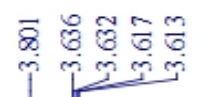

i

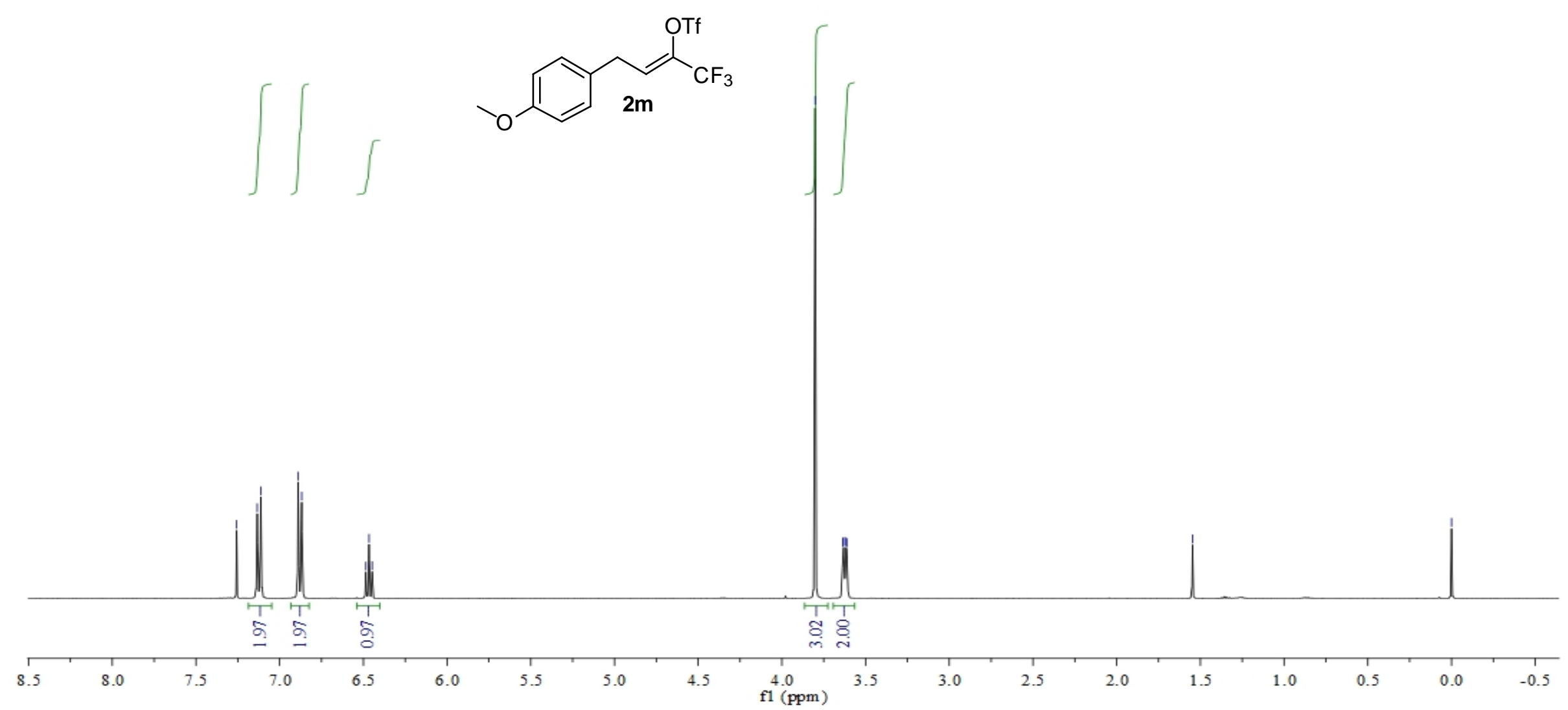


${ }^{13}$ C-NMR

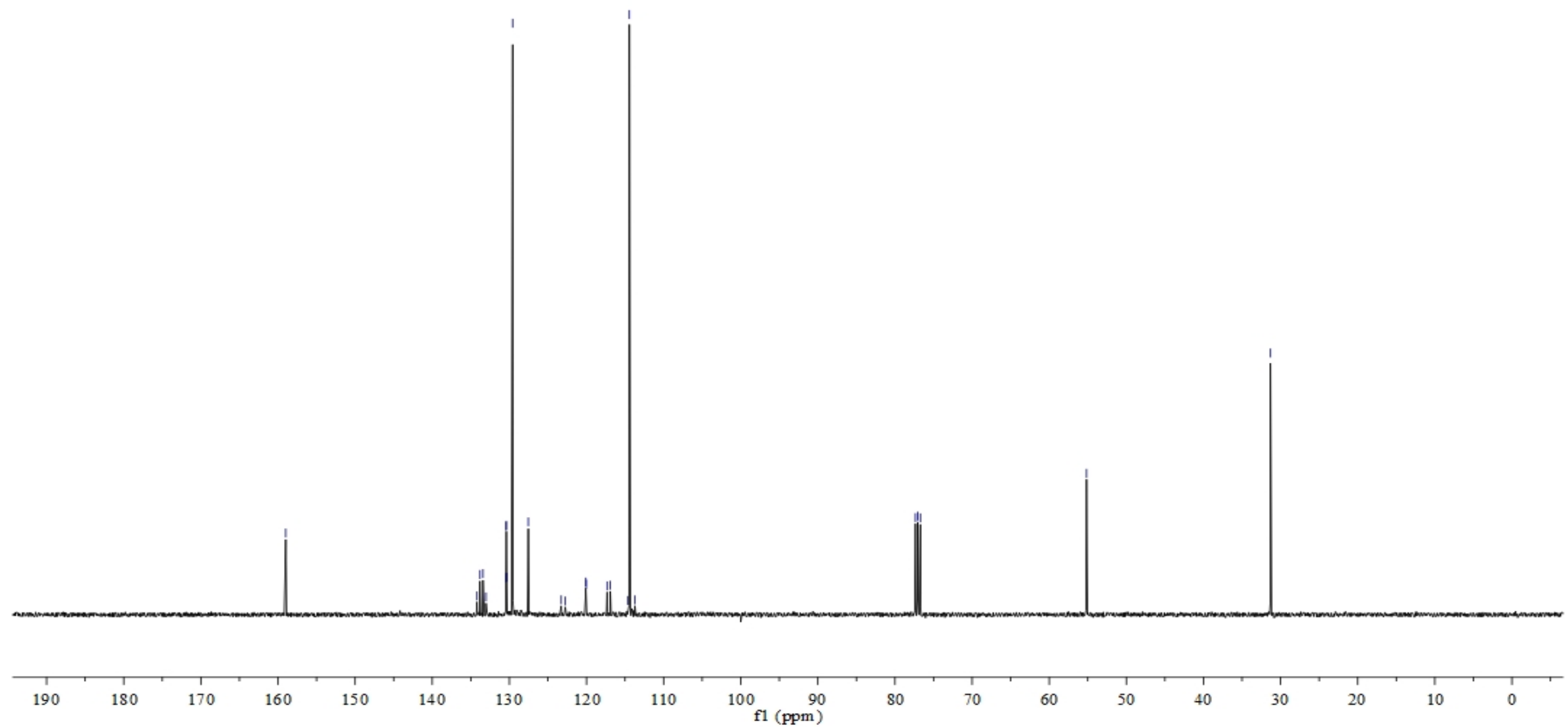


${ }^{19}$ F-NMR

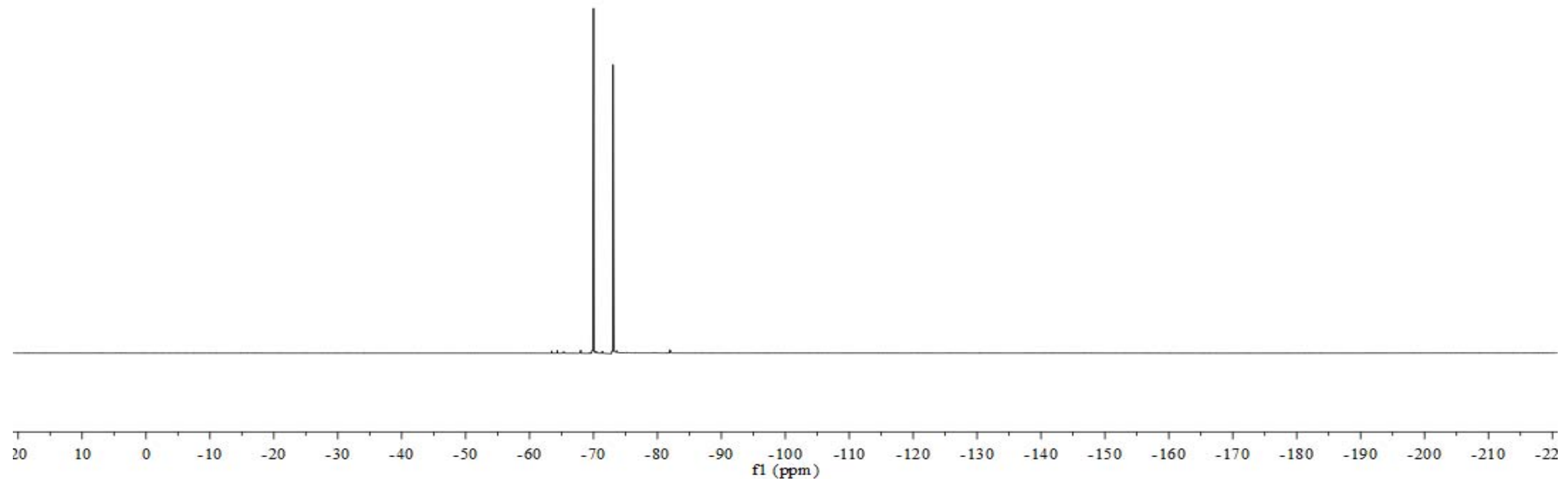



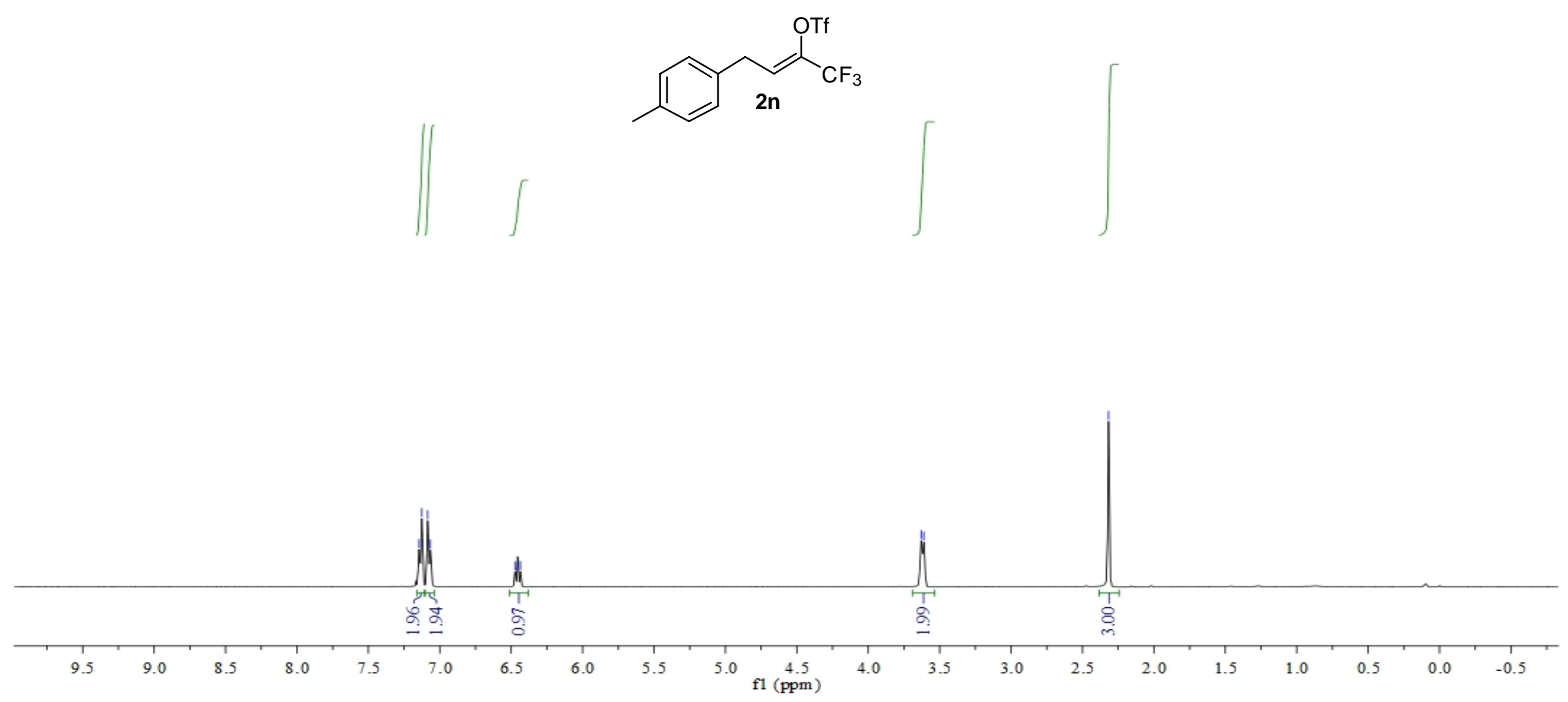


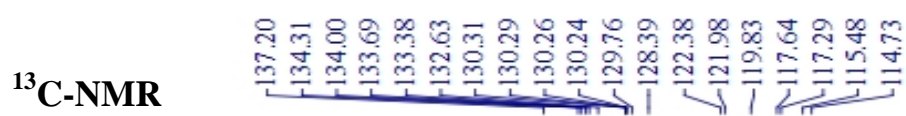

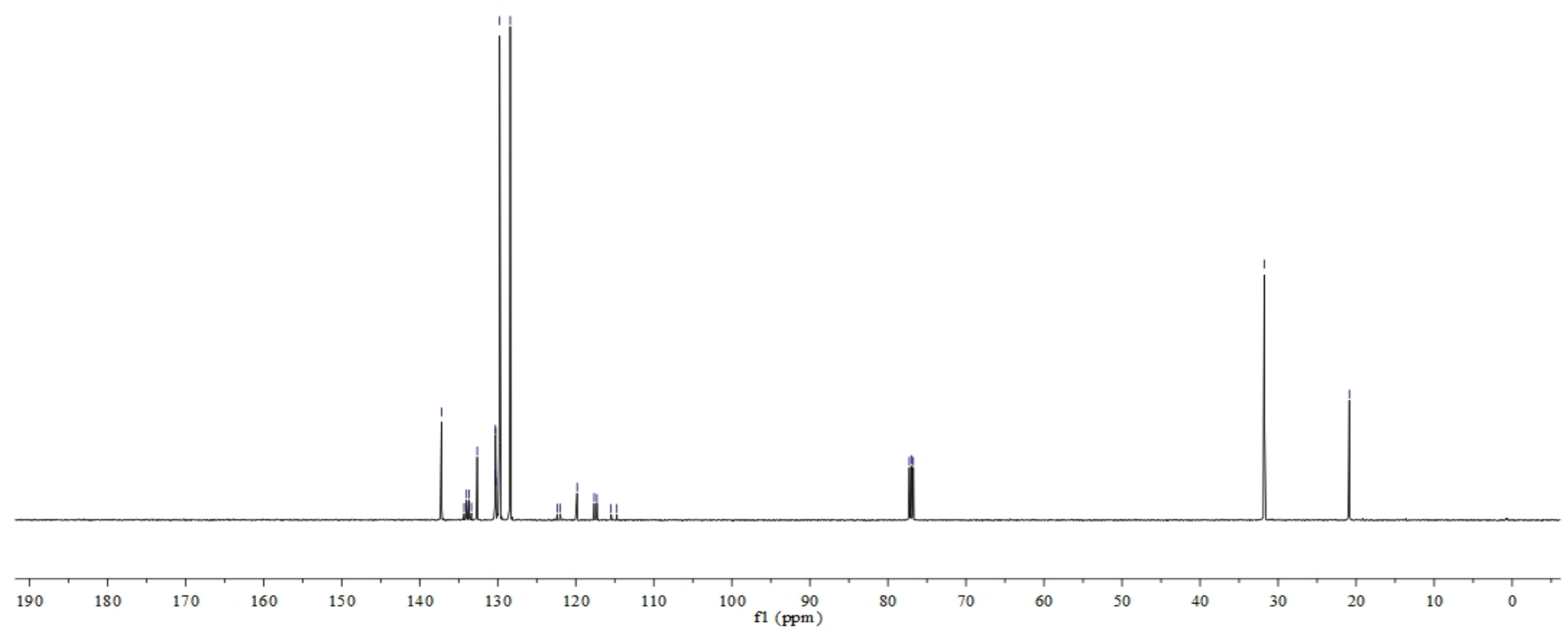


${ }^{19}$ F-NMR

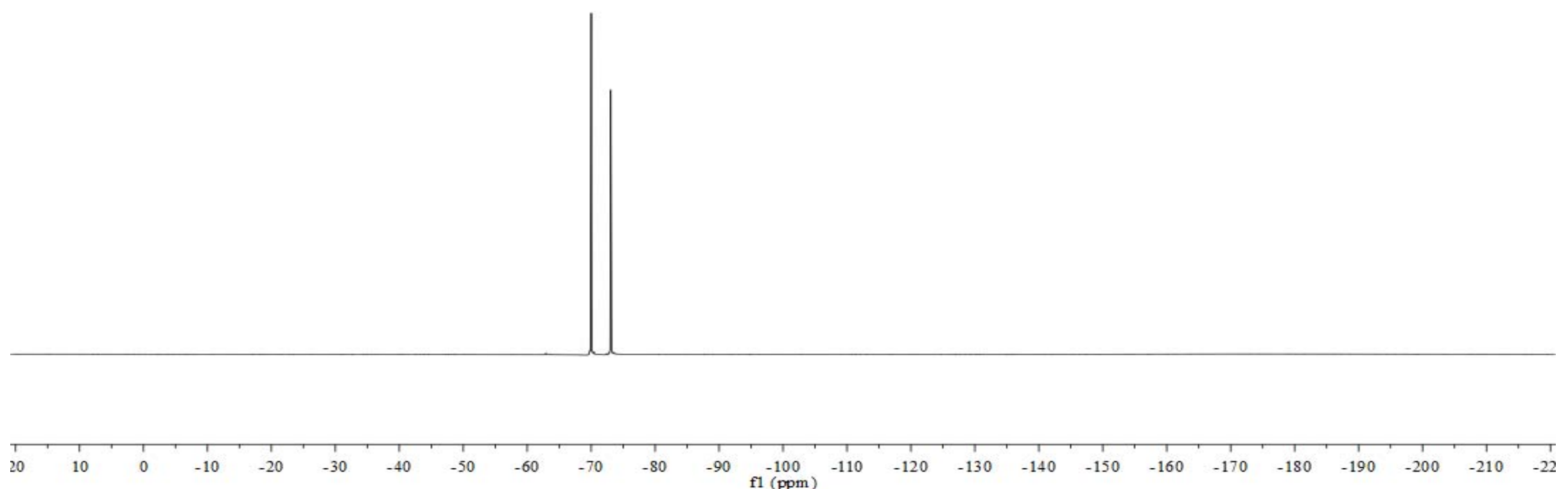


${ }^{1}$ H-NMR

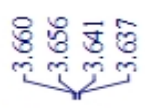
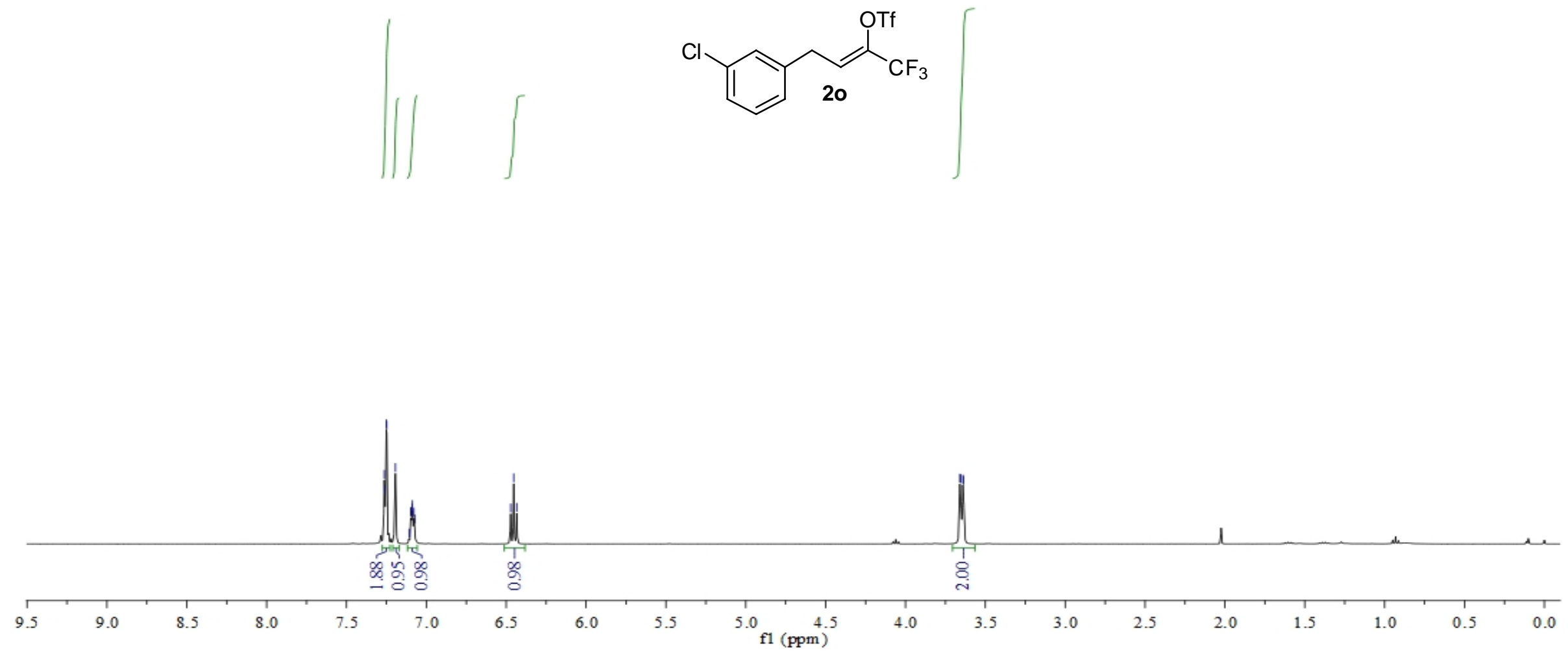


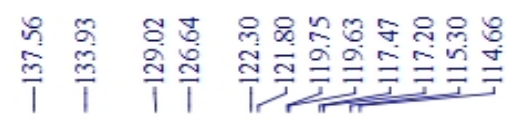

${ }^{13}$ C-NMR
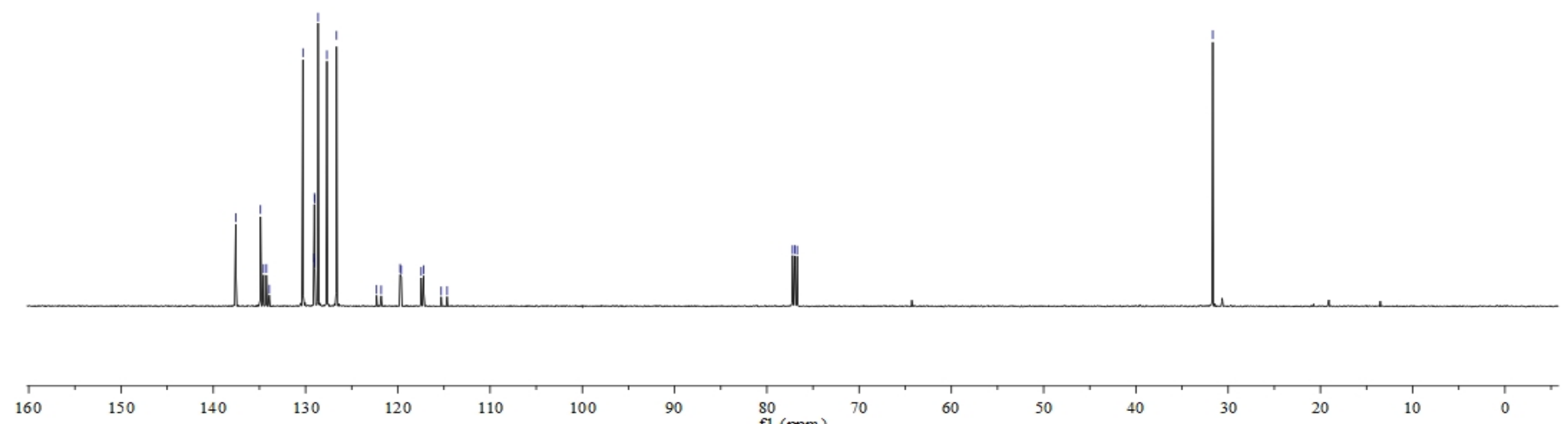

100

90

f1 (ppm)

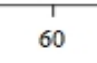

50

40 30 20 
${ }^{19}$ F-NMR

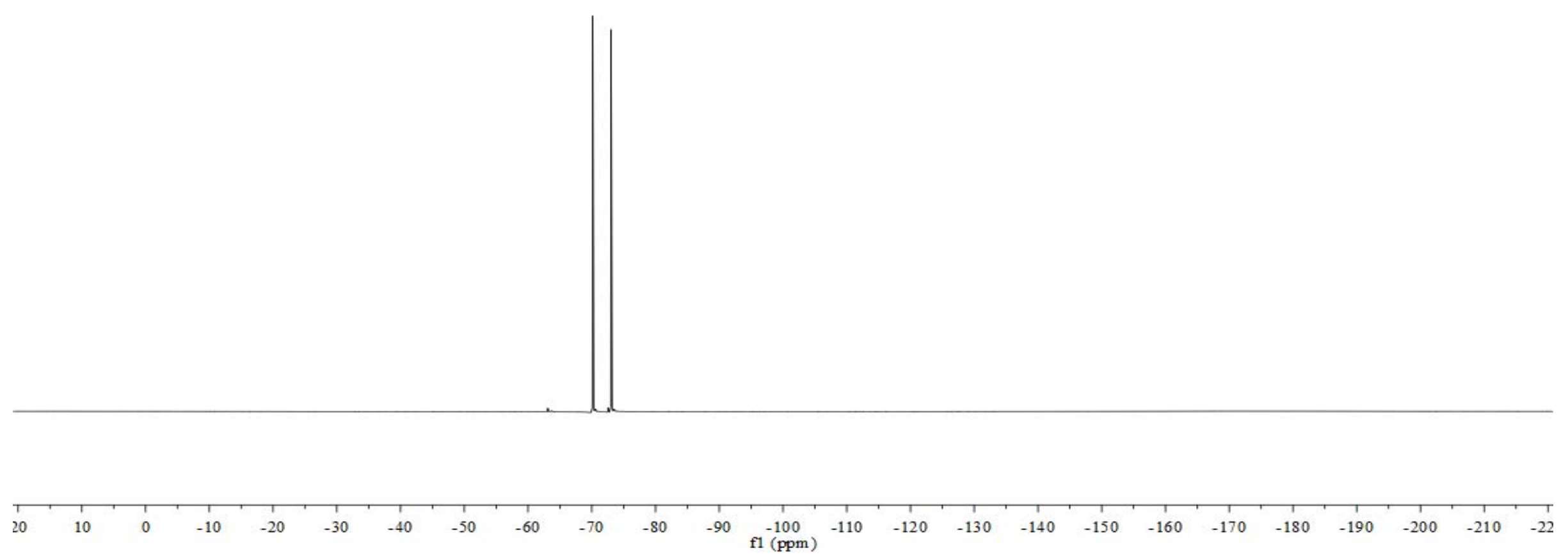



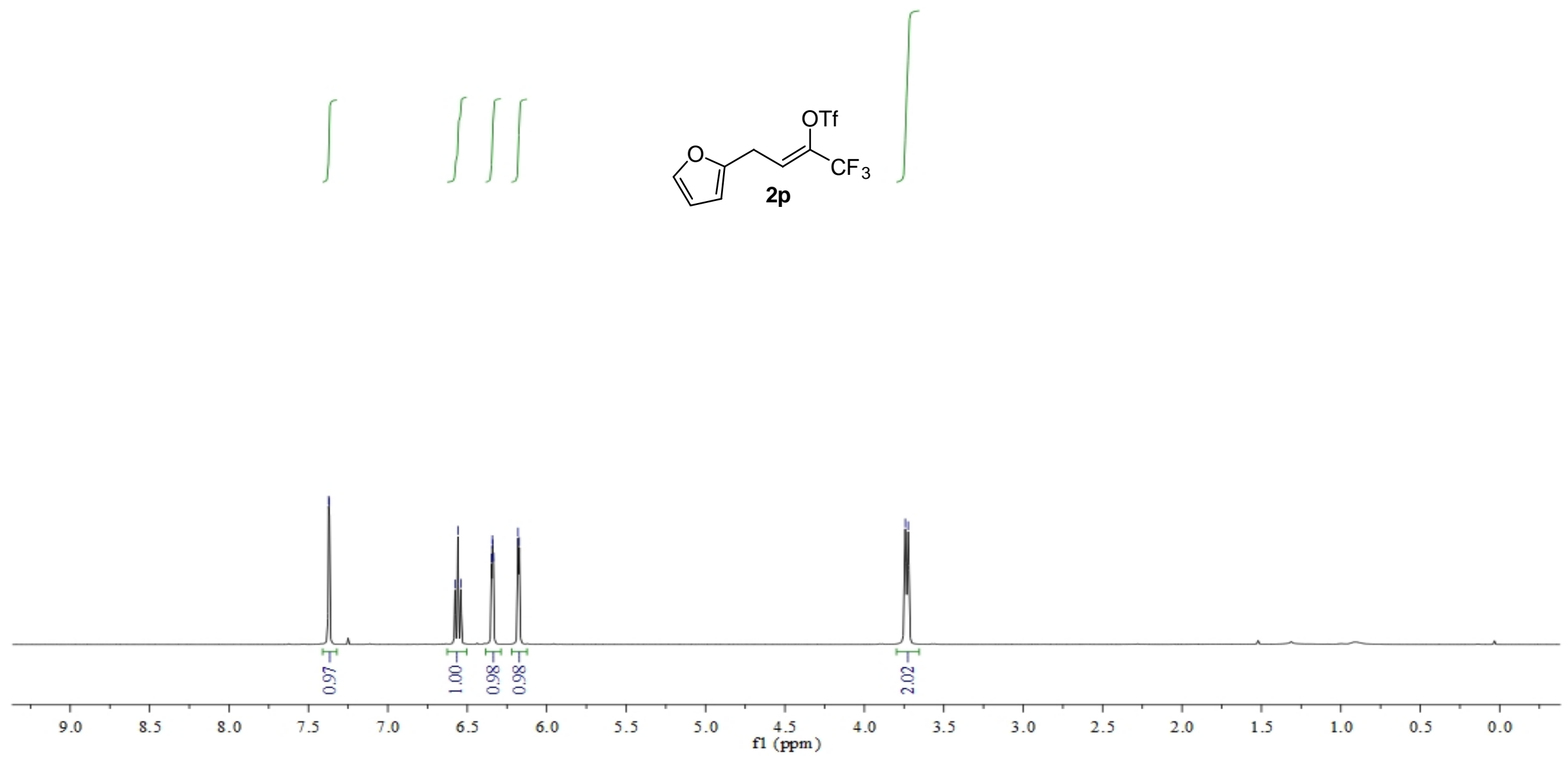
${ }^{13}$ C-NMR

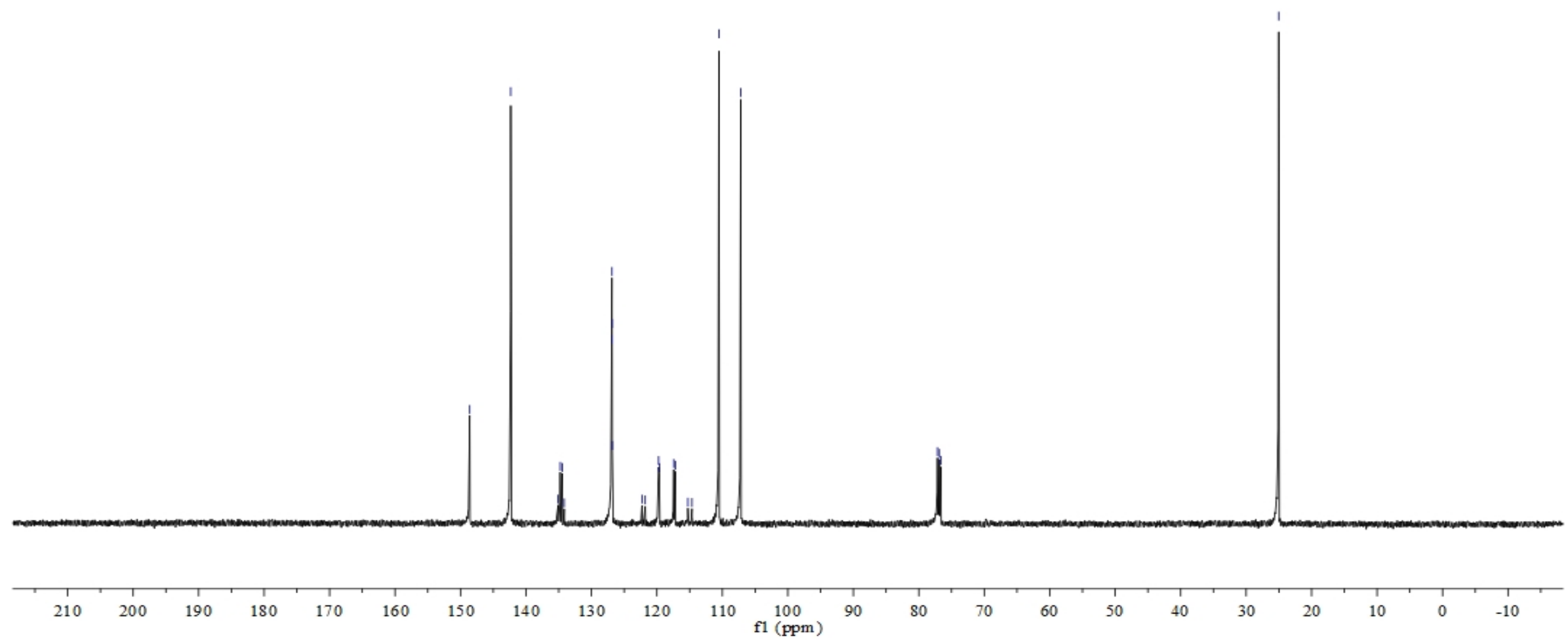


${ }^{19}$ F-NMR

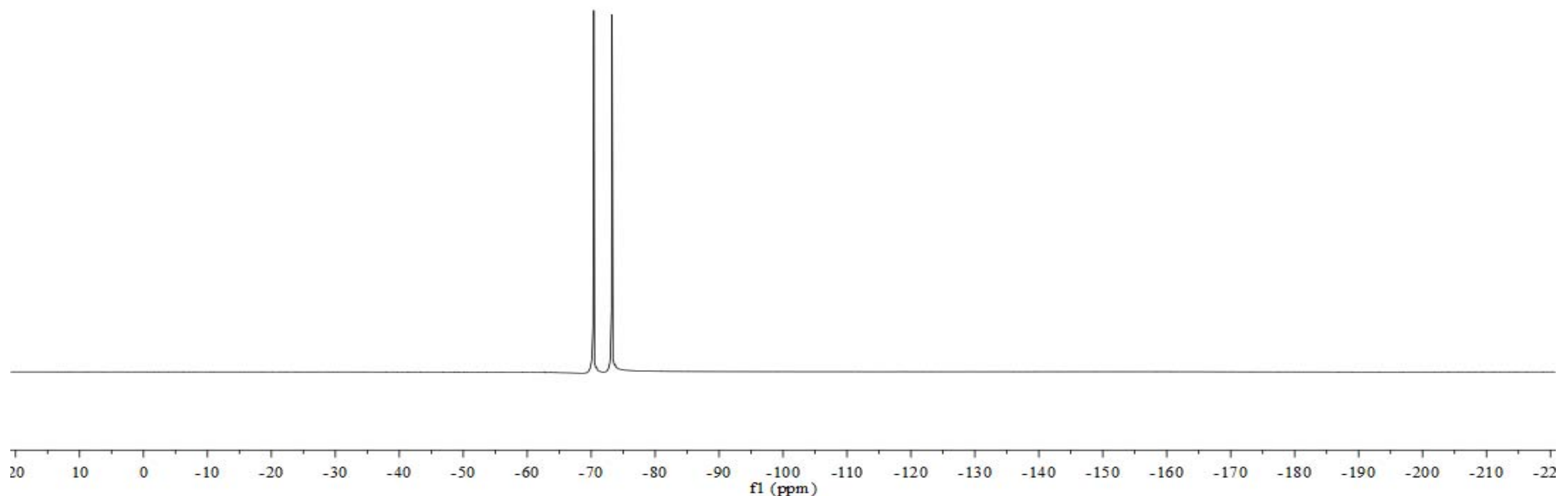




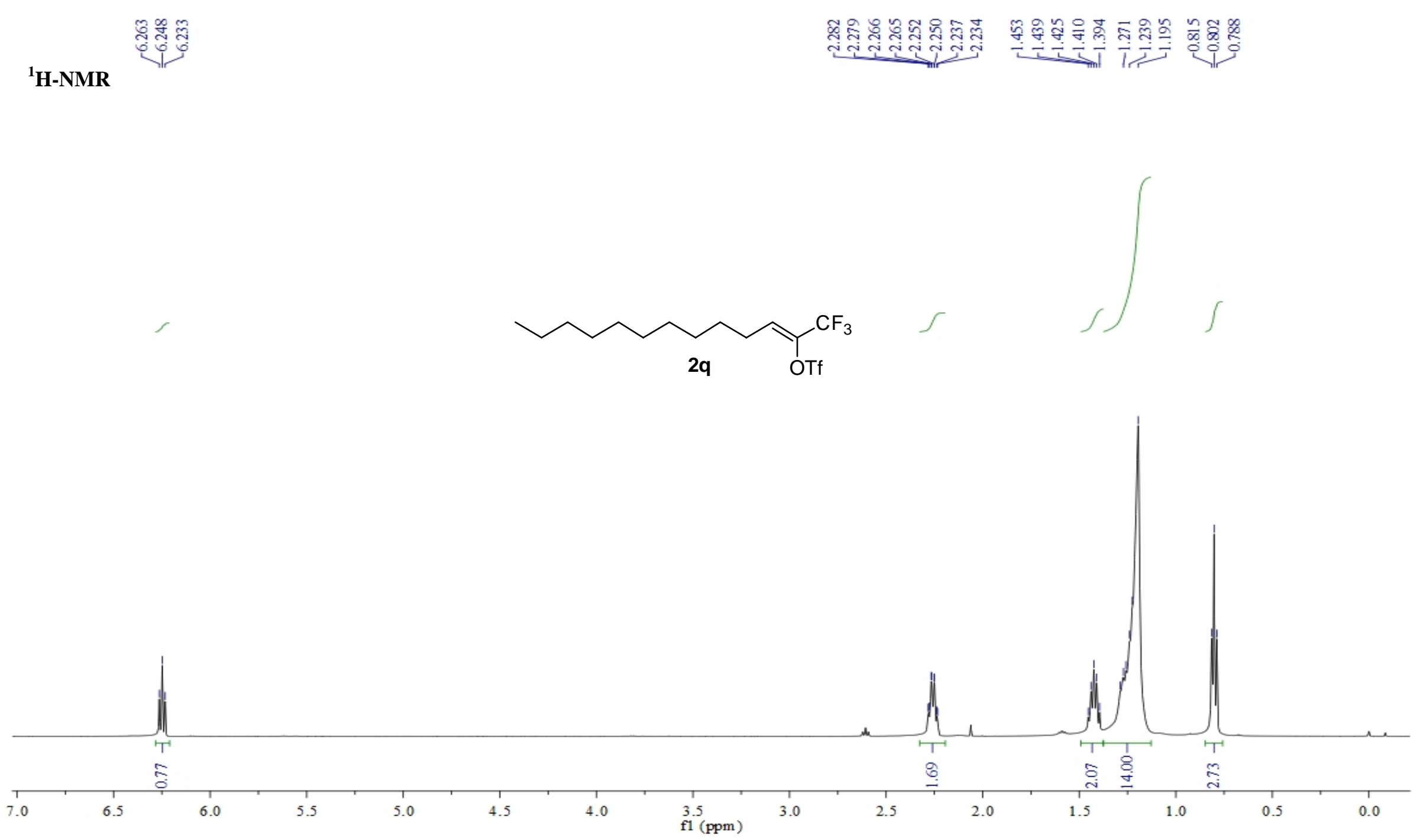




\section{${ }^{13}$ C-NMR

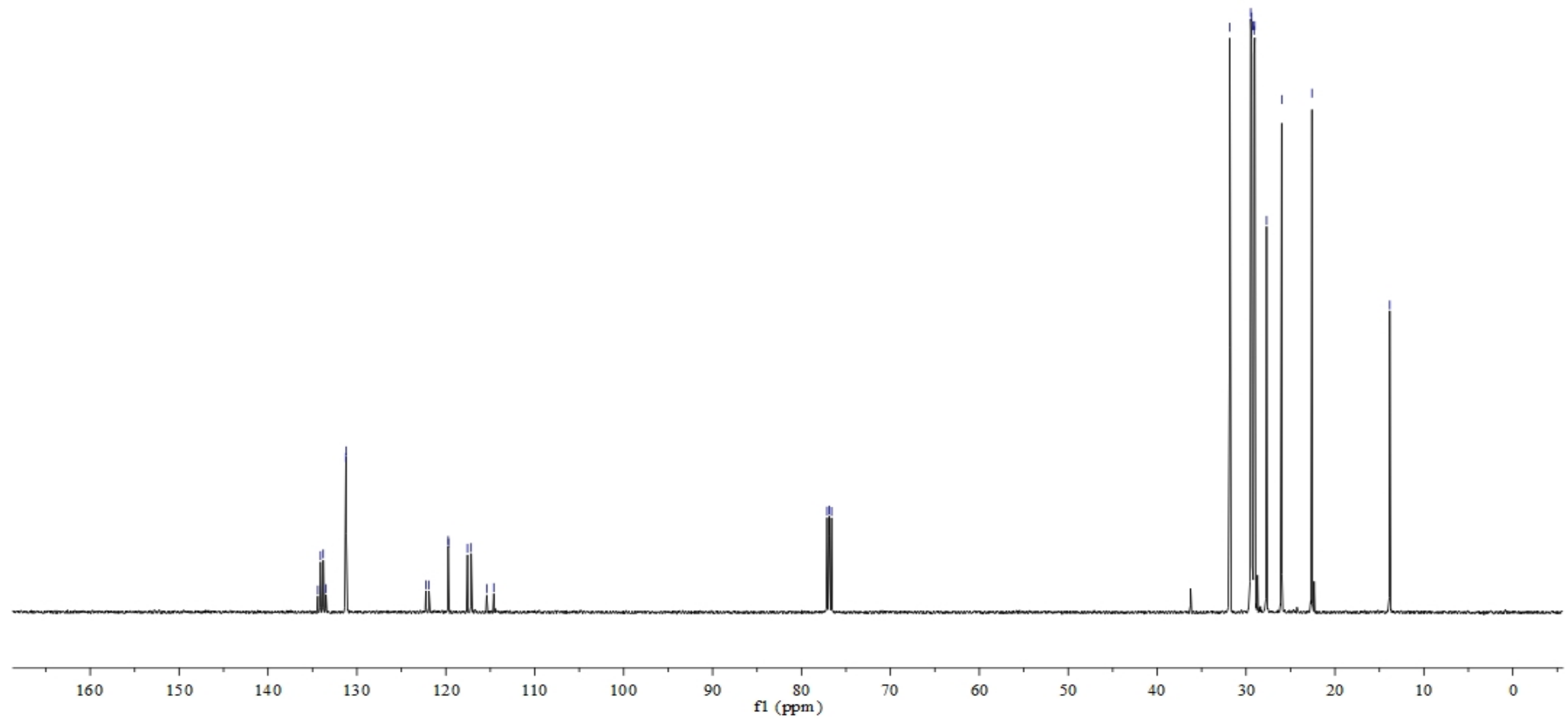


${ }^{19}$ F-NMR

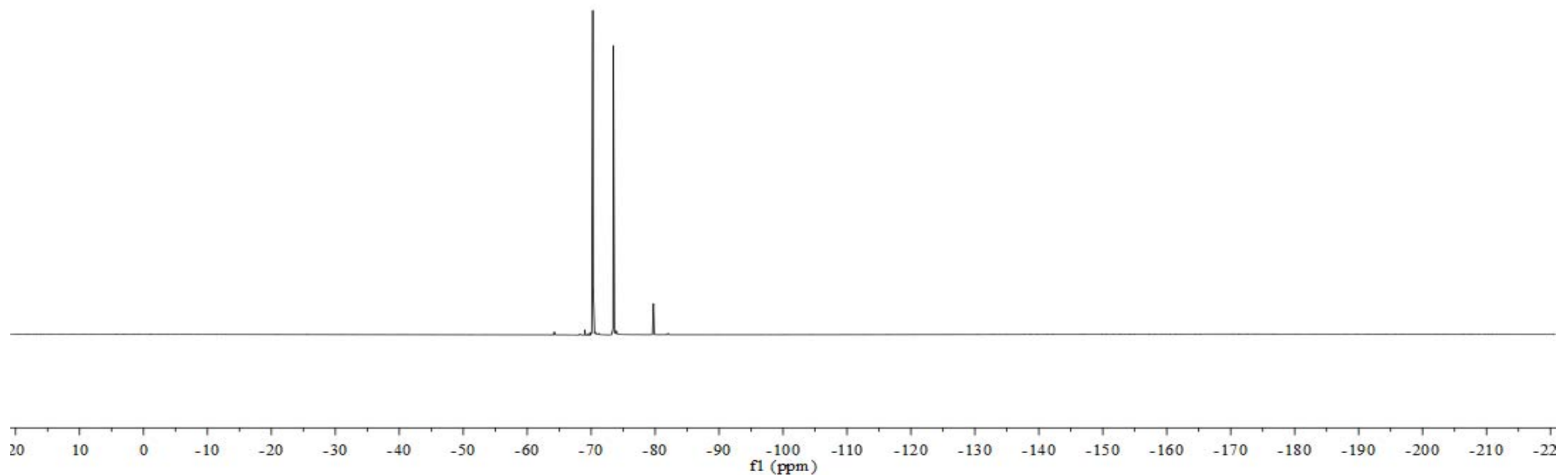




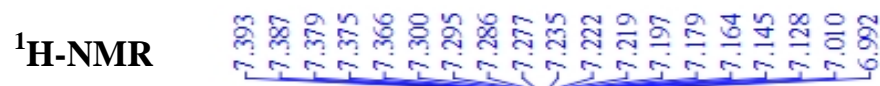

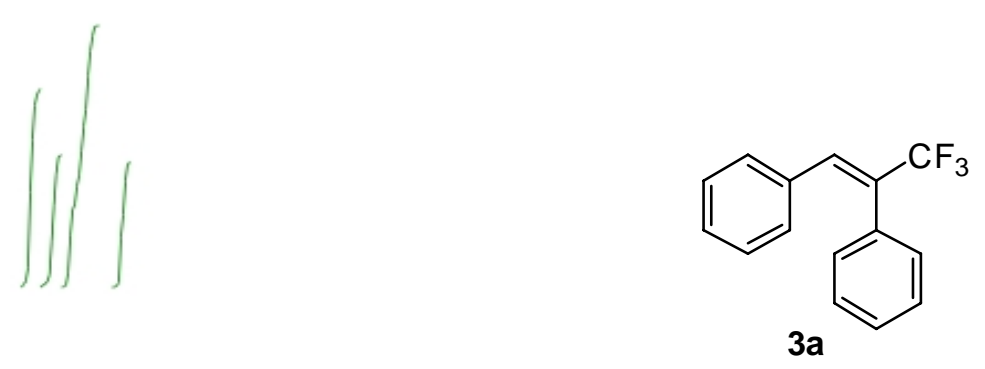

$\stackrel{8}{\mathbb{9}}$

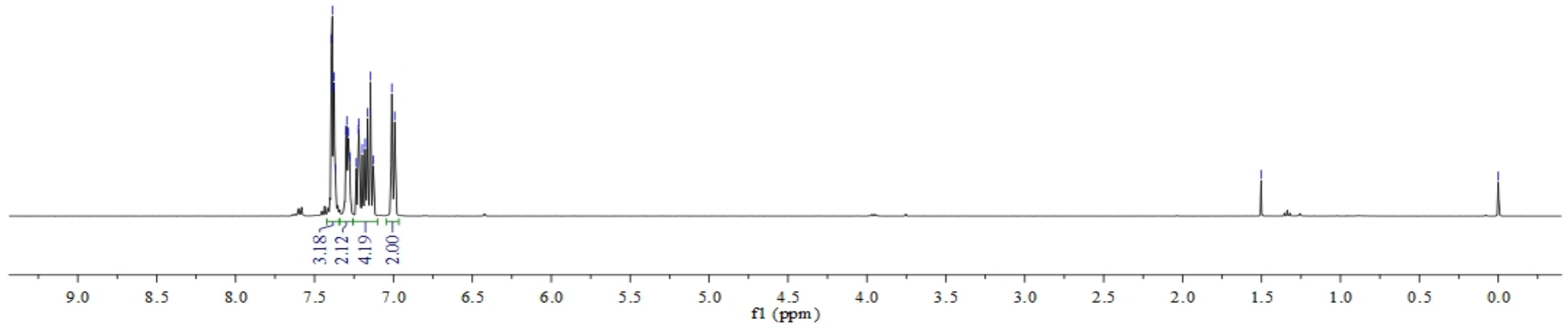




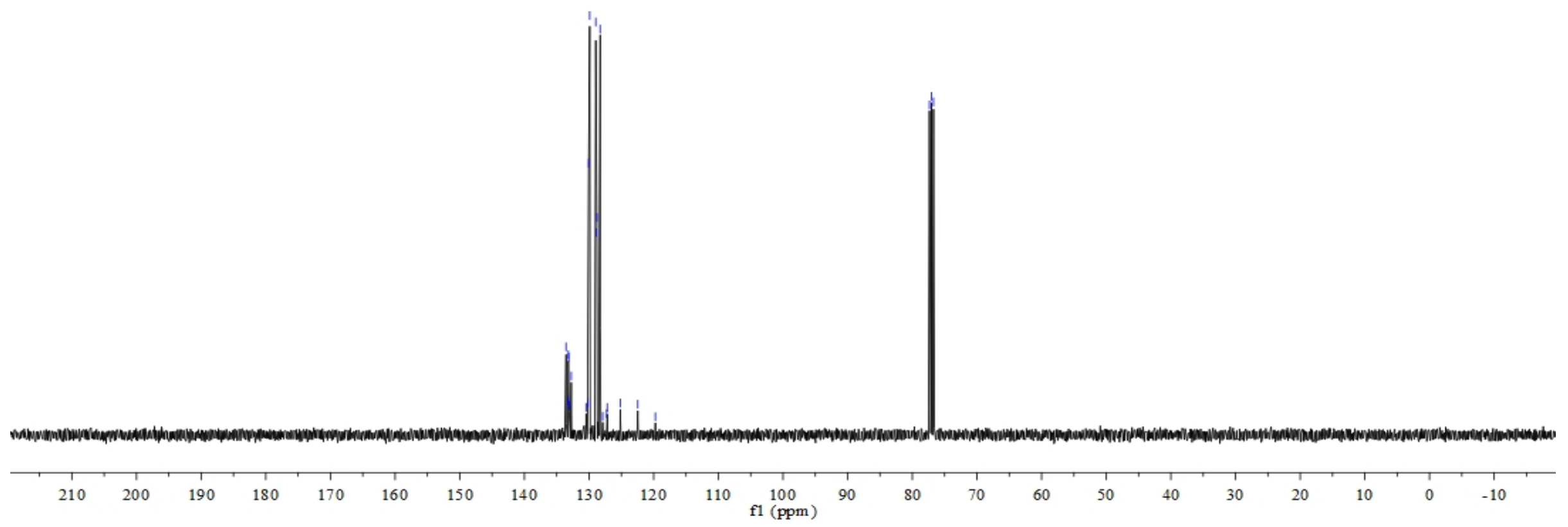


${ }^{19}$ F-NMR

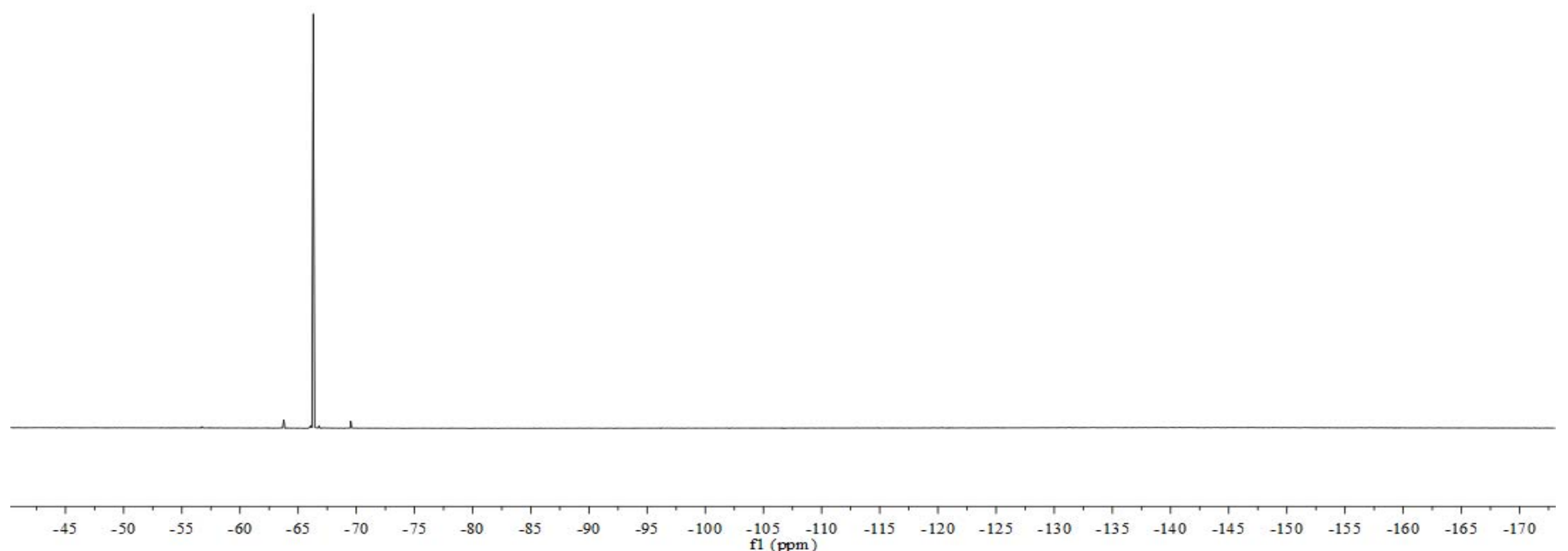



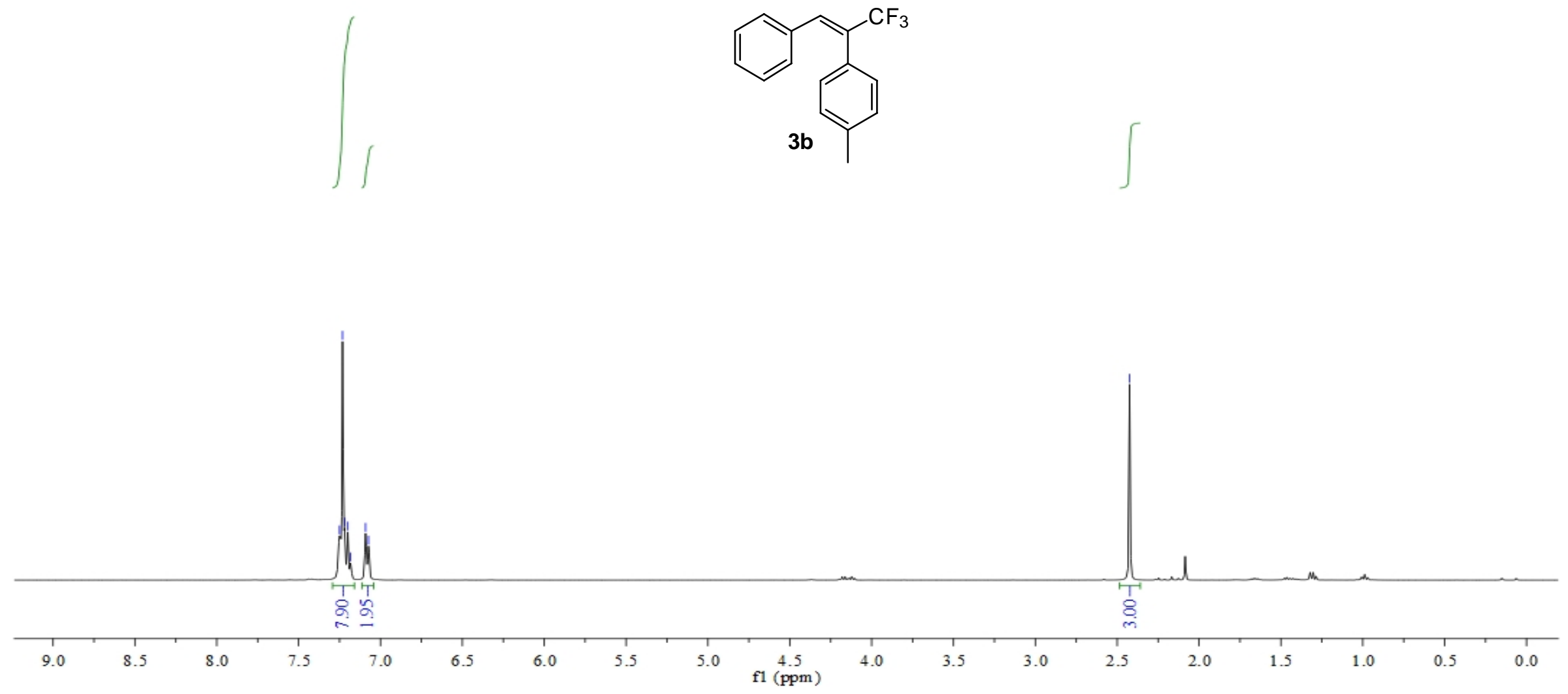
${ }^{13}$ C-NMR
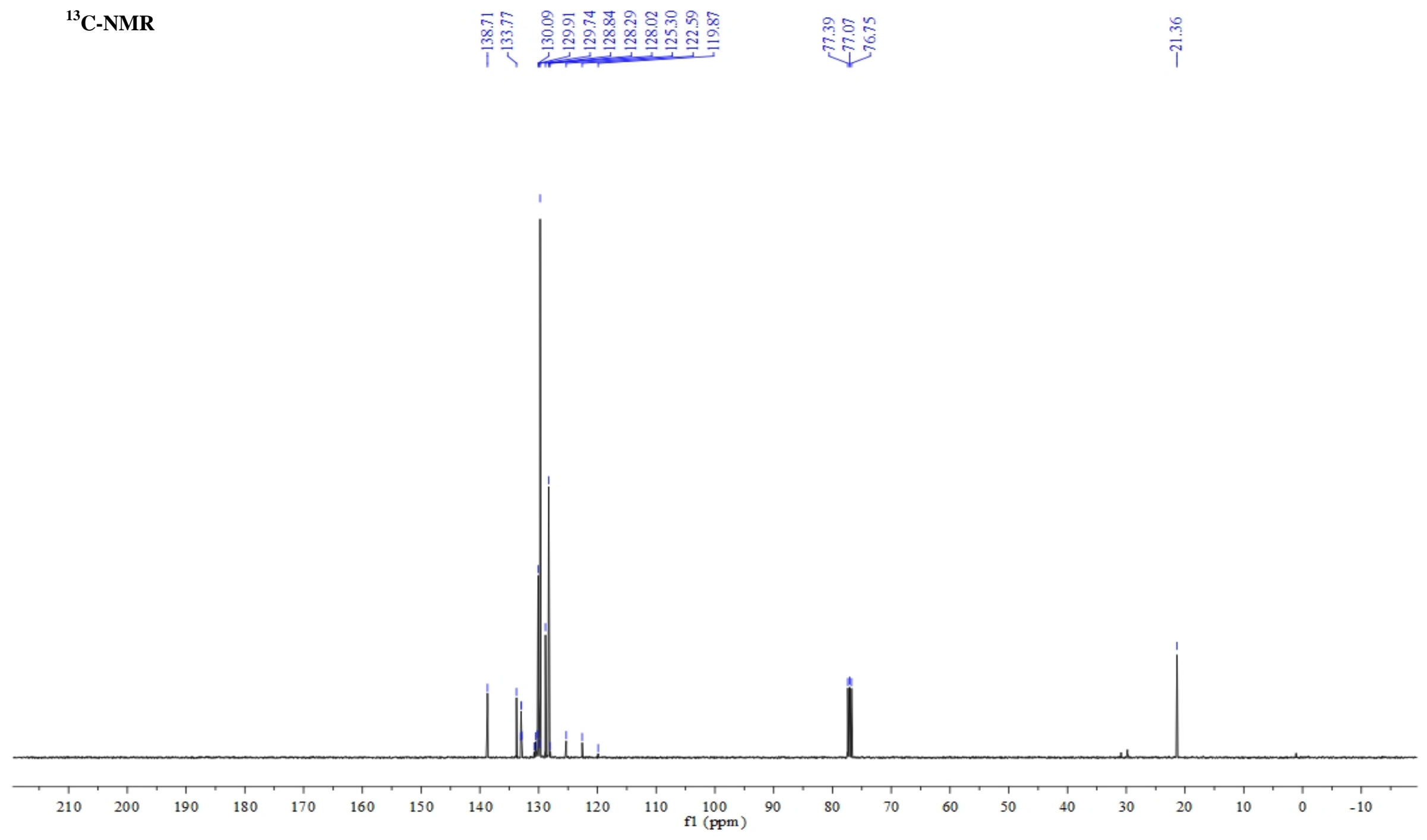
${ }^{19}$ F-NMR

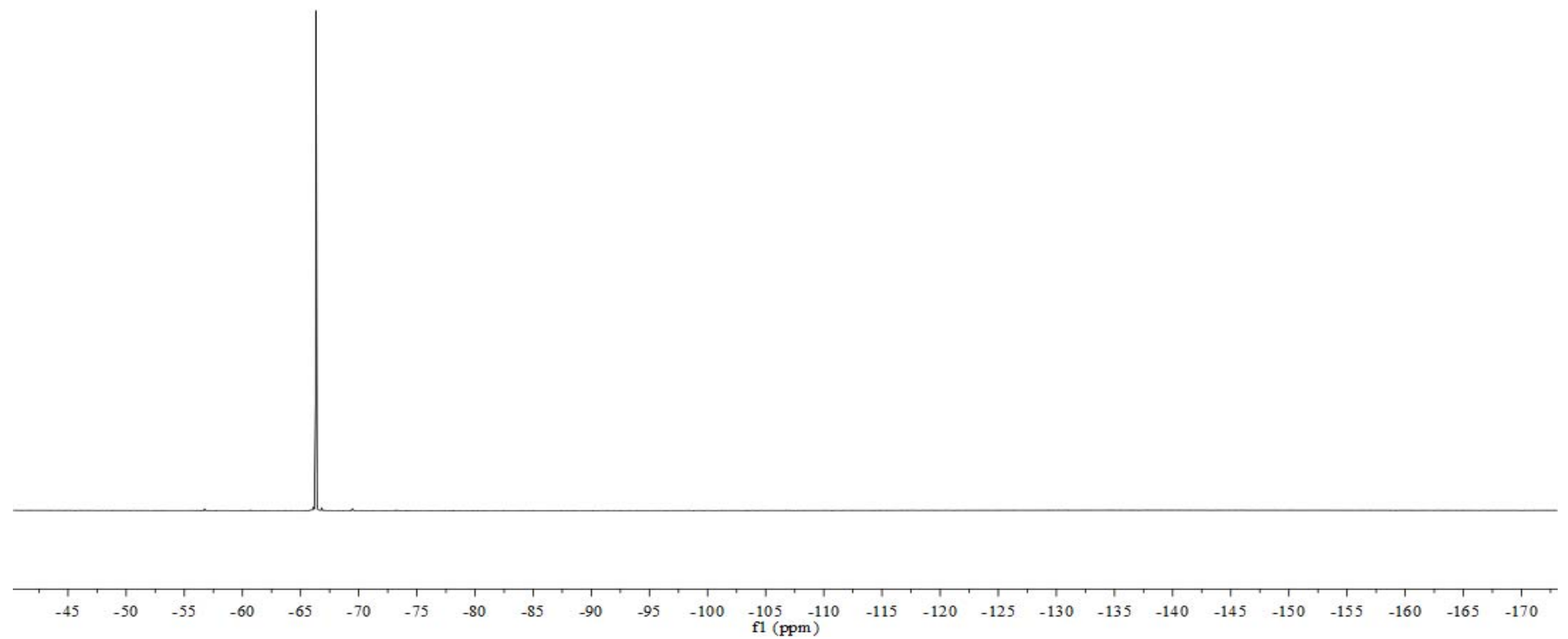




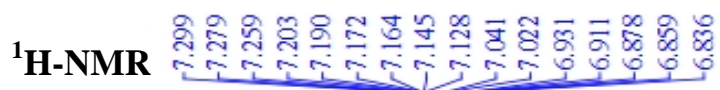
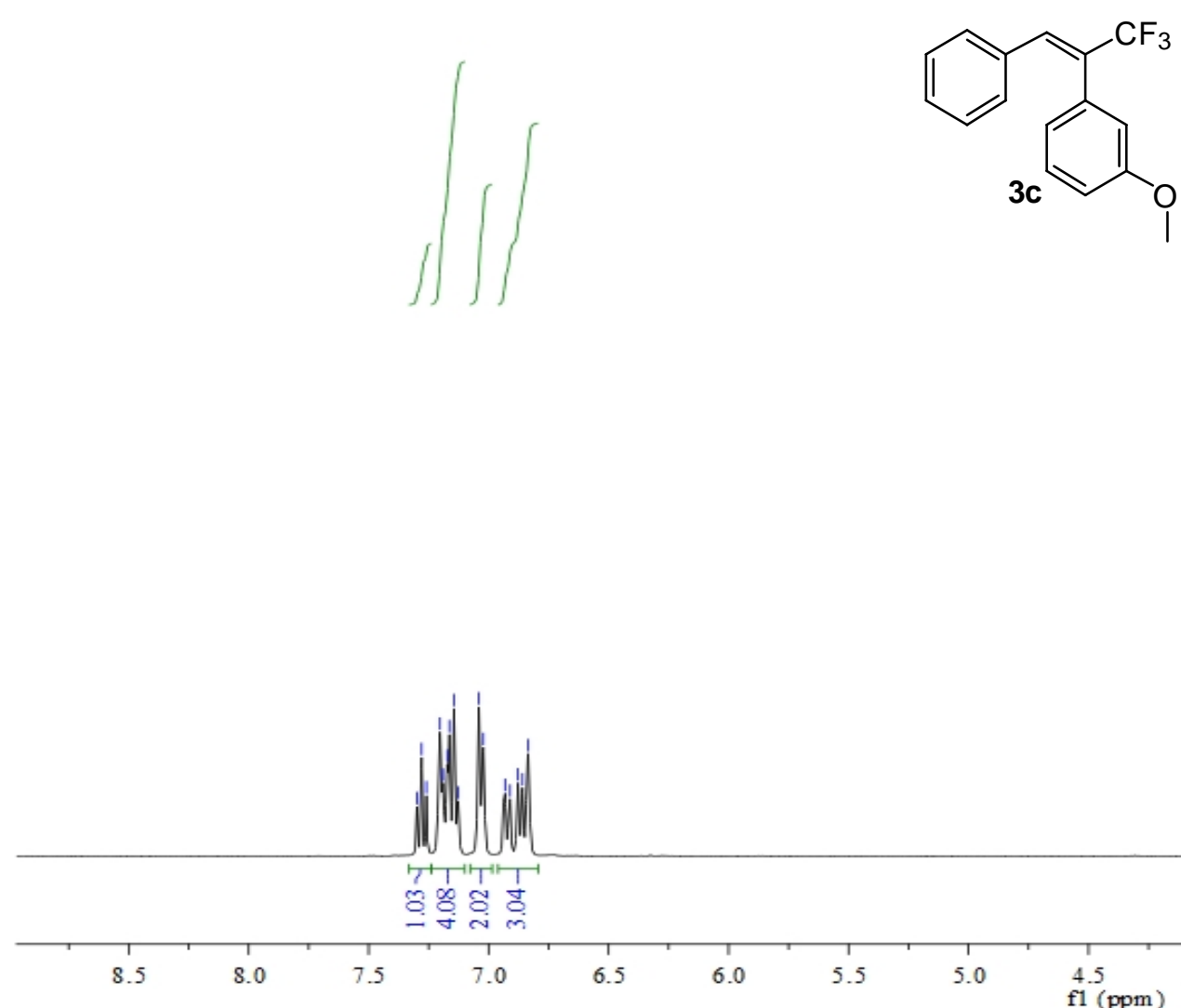

5.0

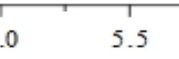

4.5
f1 $(\mathrm{ppm})$

$3.5 \quad 3.0$
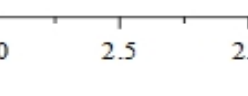
${ }^{13}$ C-NMR

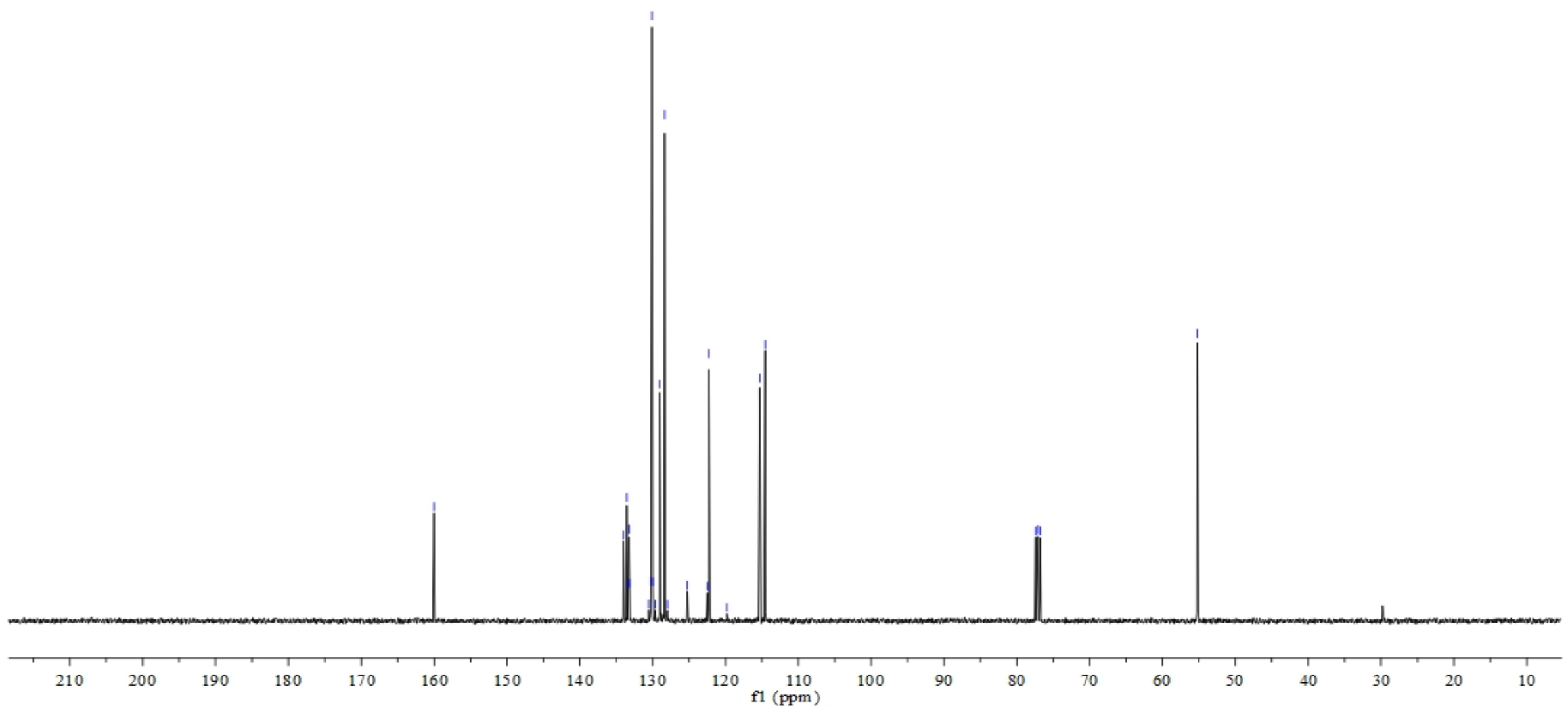


${ }^{19}$ F-NMR

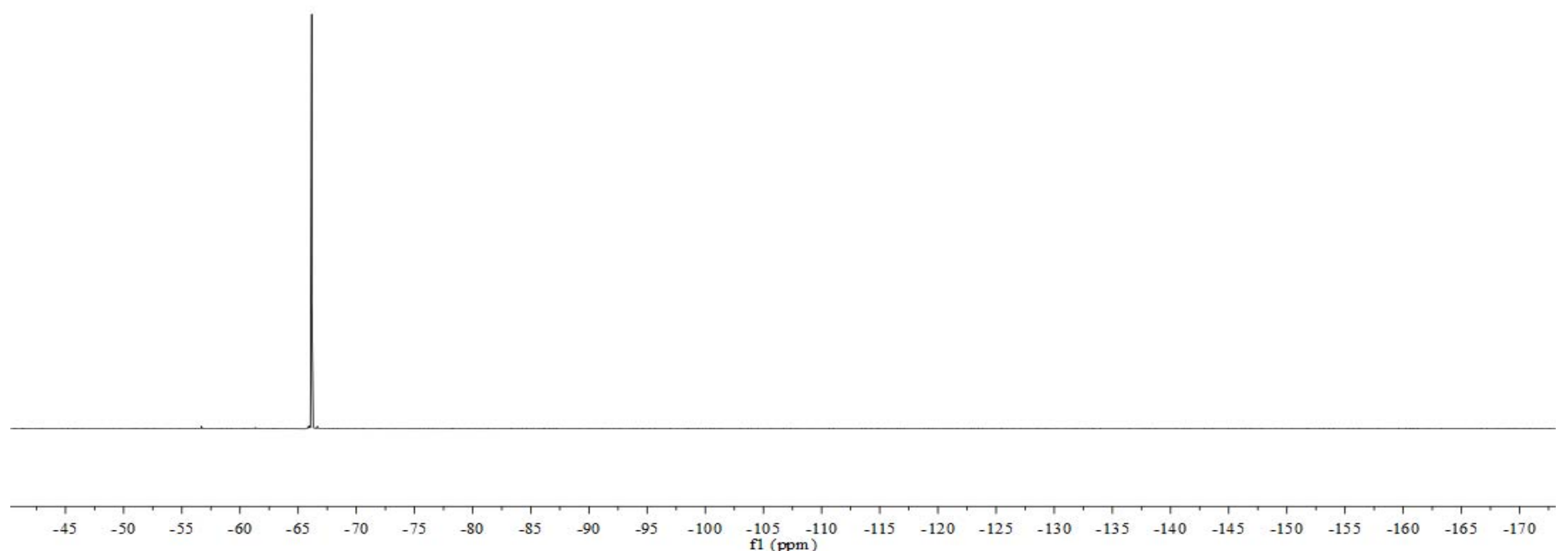


${ }^{1}$ H-NMR

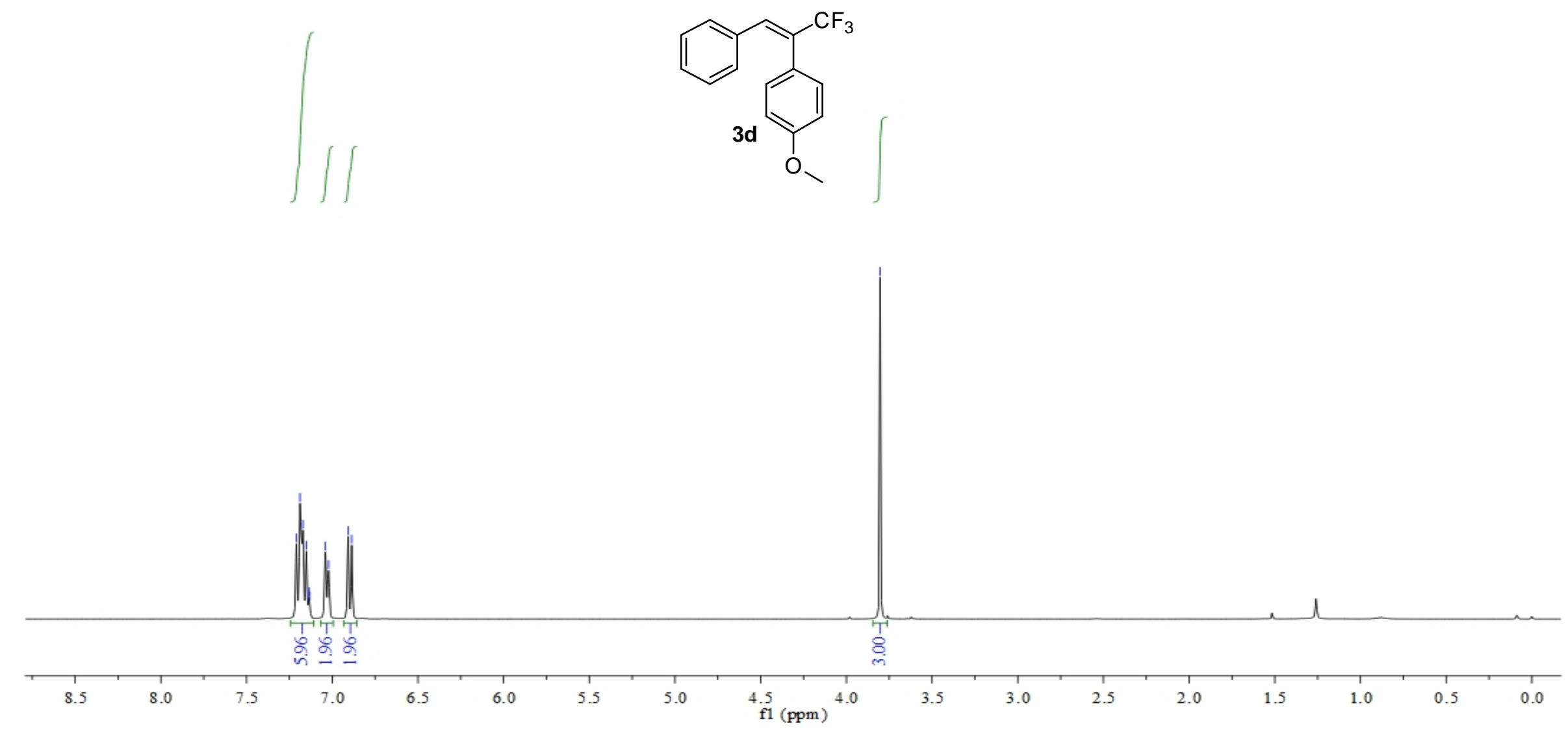


${ }^{13}$ C-NMR

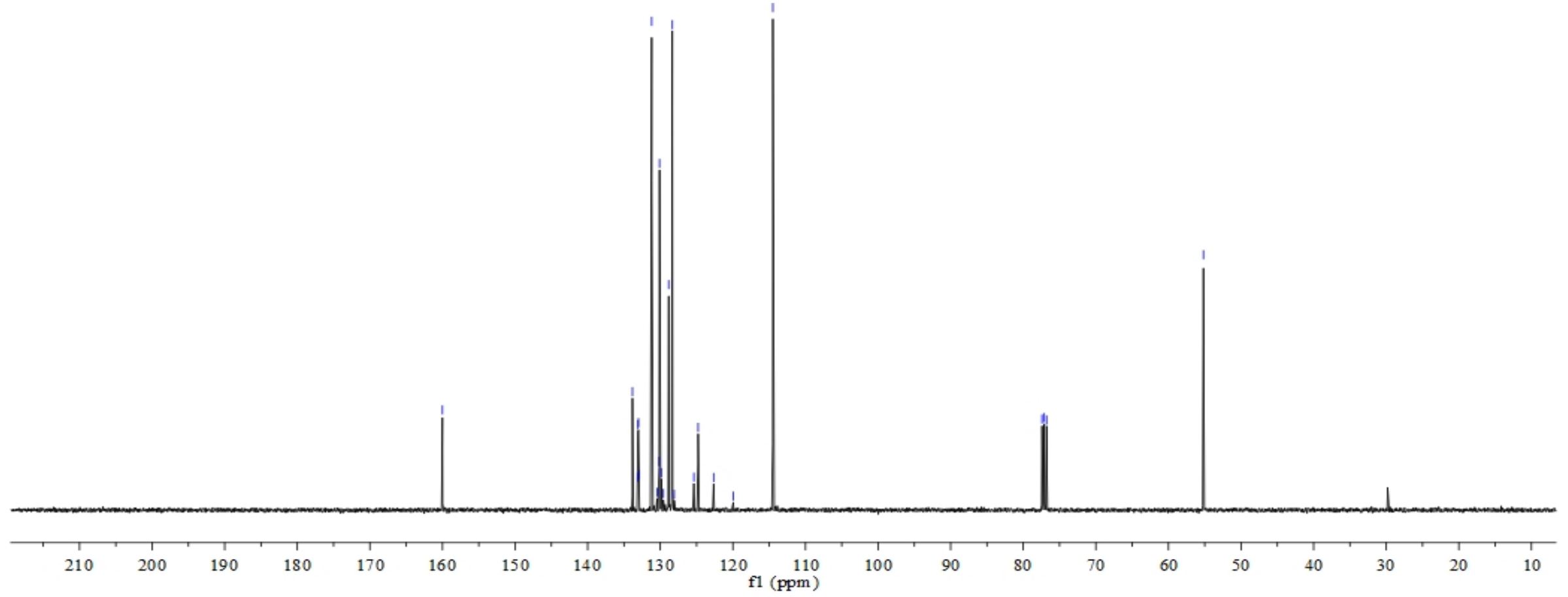


${ }^{19}$ F-NMR

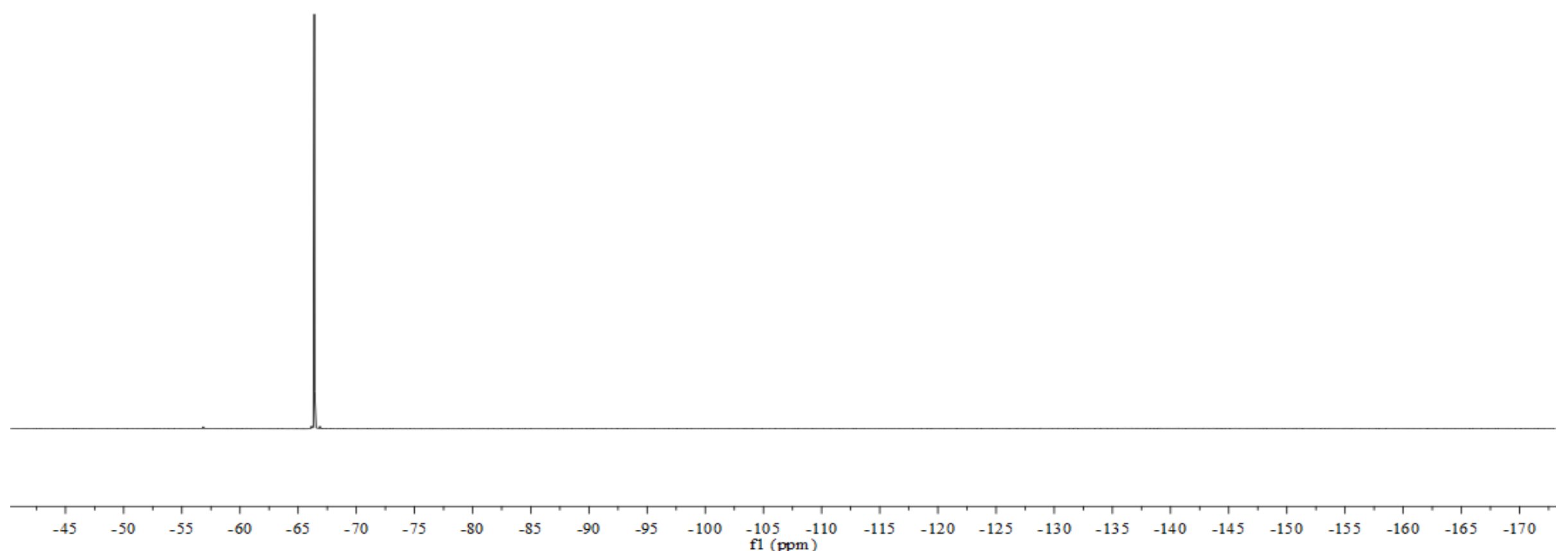




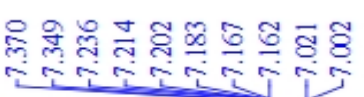

${ }^{1}$ H-NMR
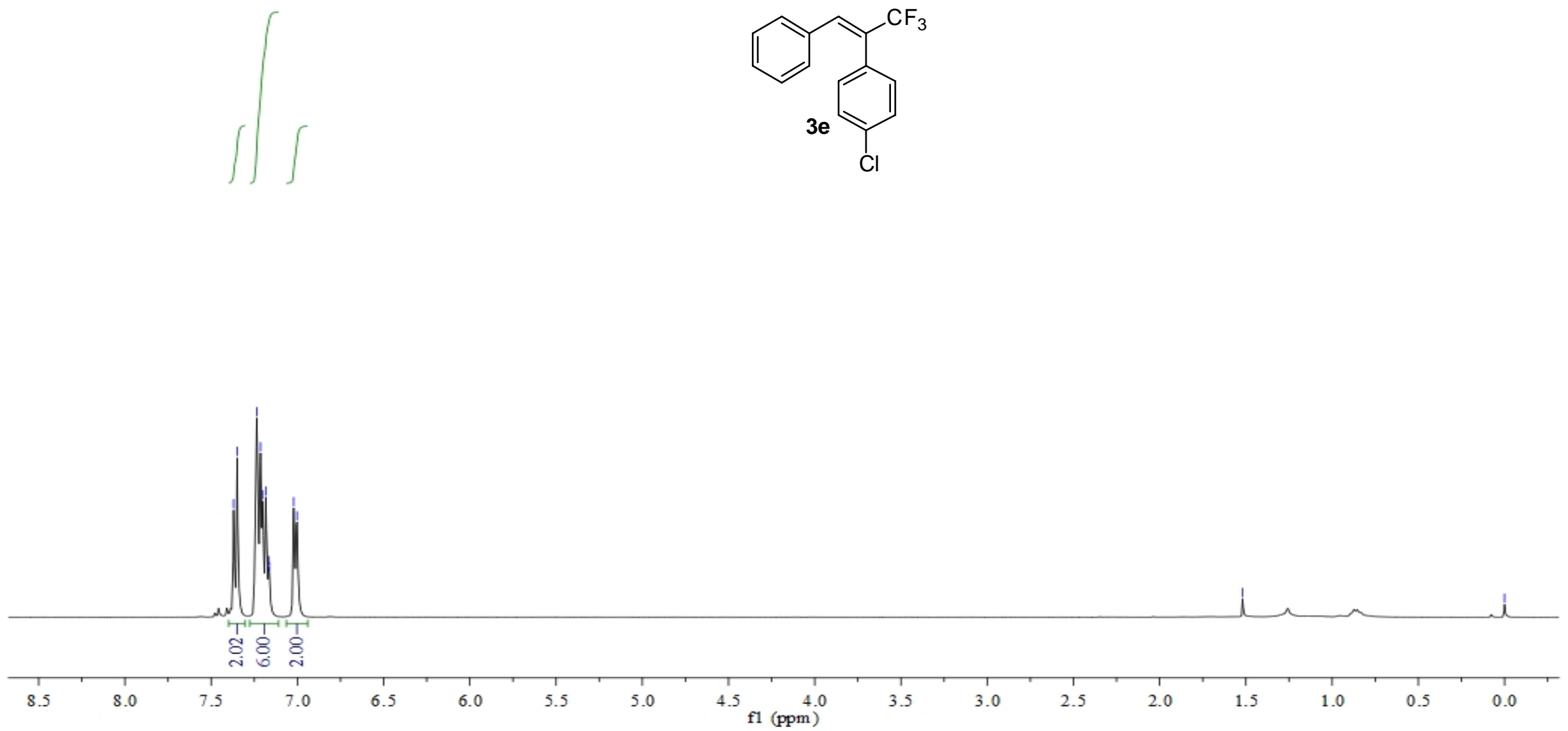


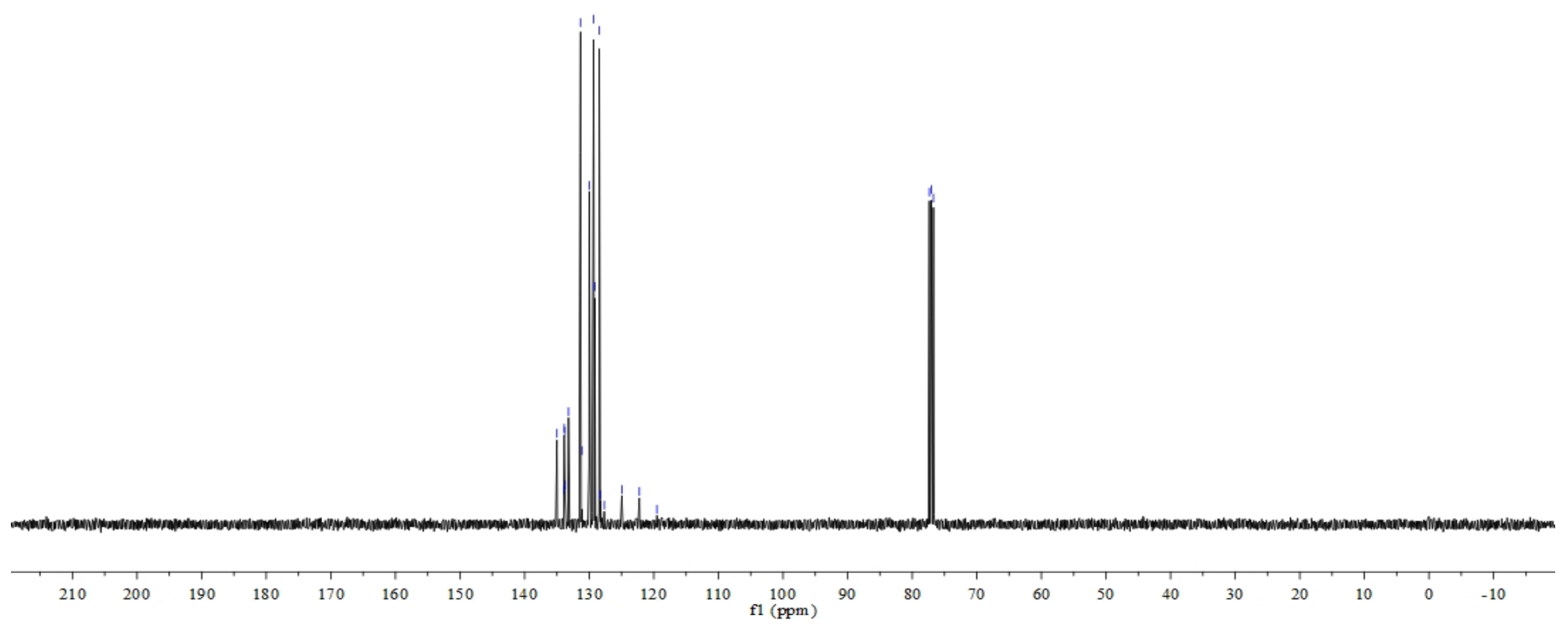


${ }^{19}$ F-NMR

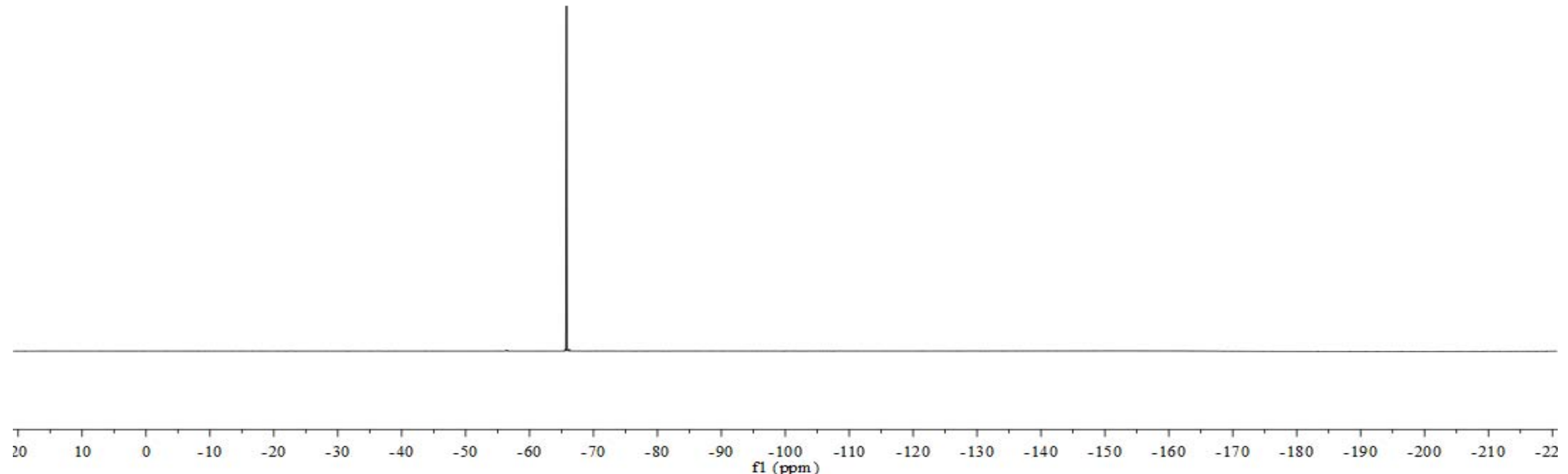



MR

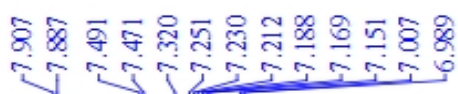
${ }^{1} \mathrm{H}$-NMR
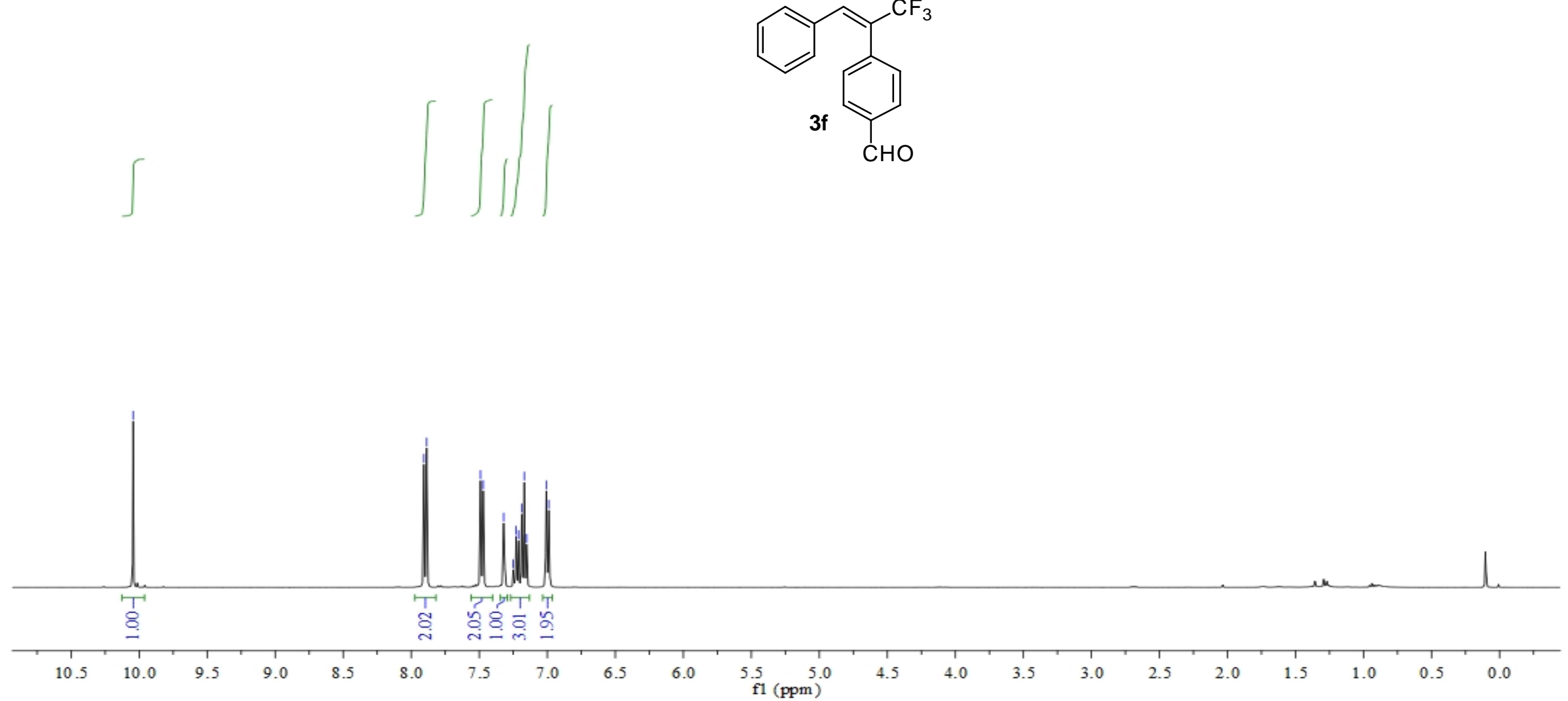
${ }^{13} \mathrm{C}-\mathrm{NMR}$

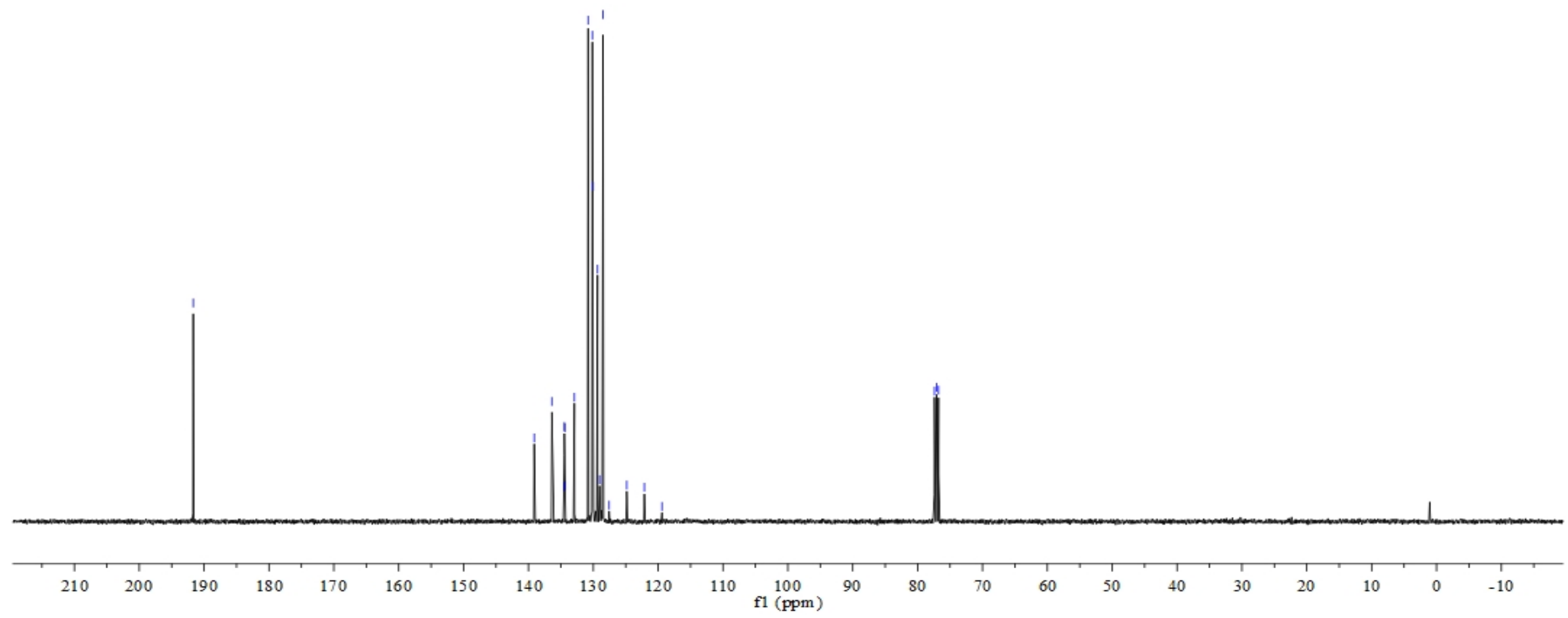


${ }^{19}$ F-NMR

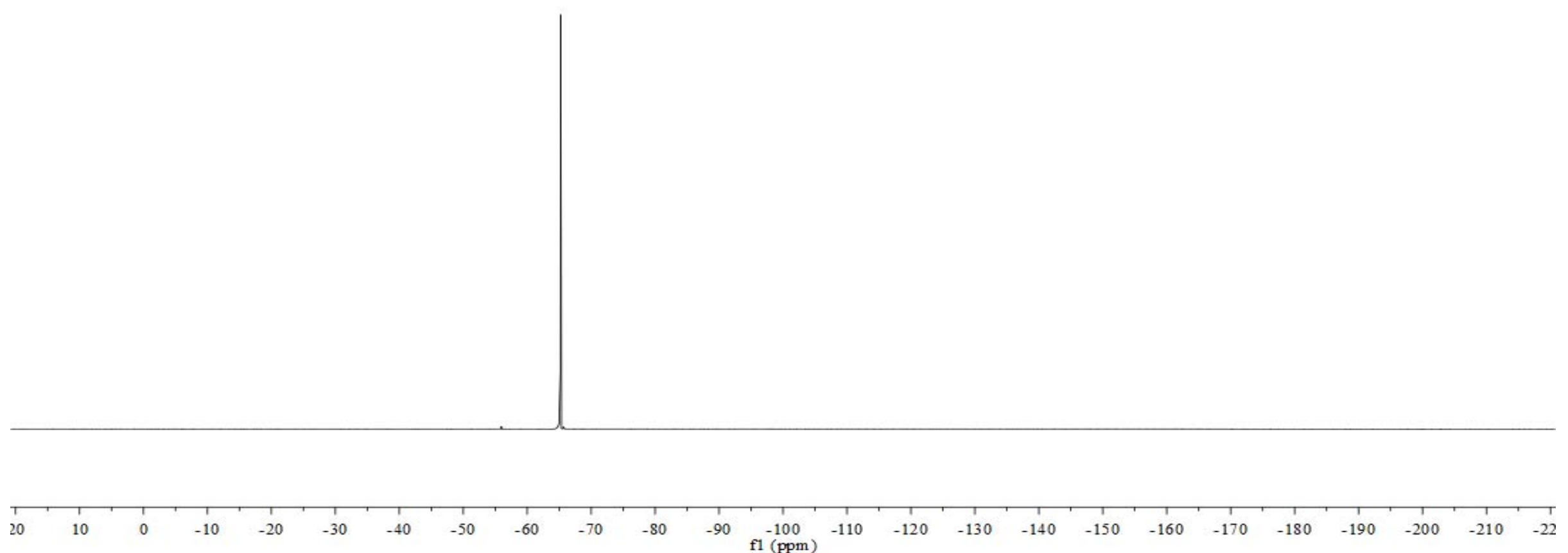




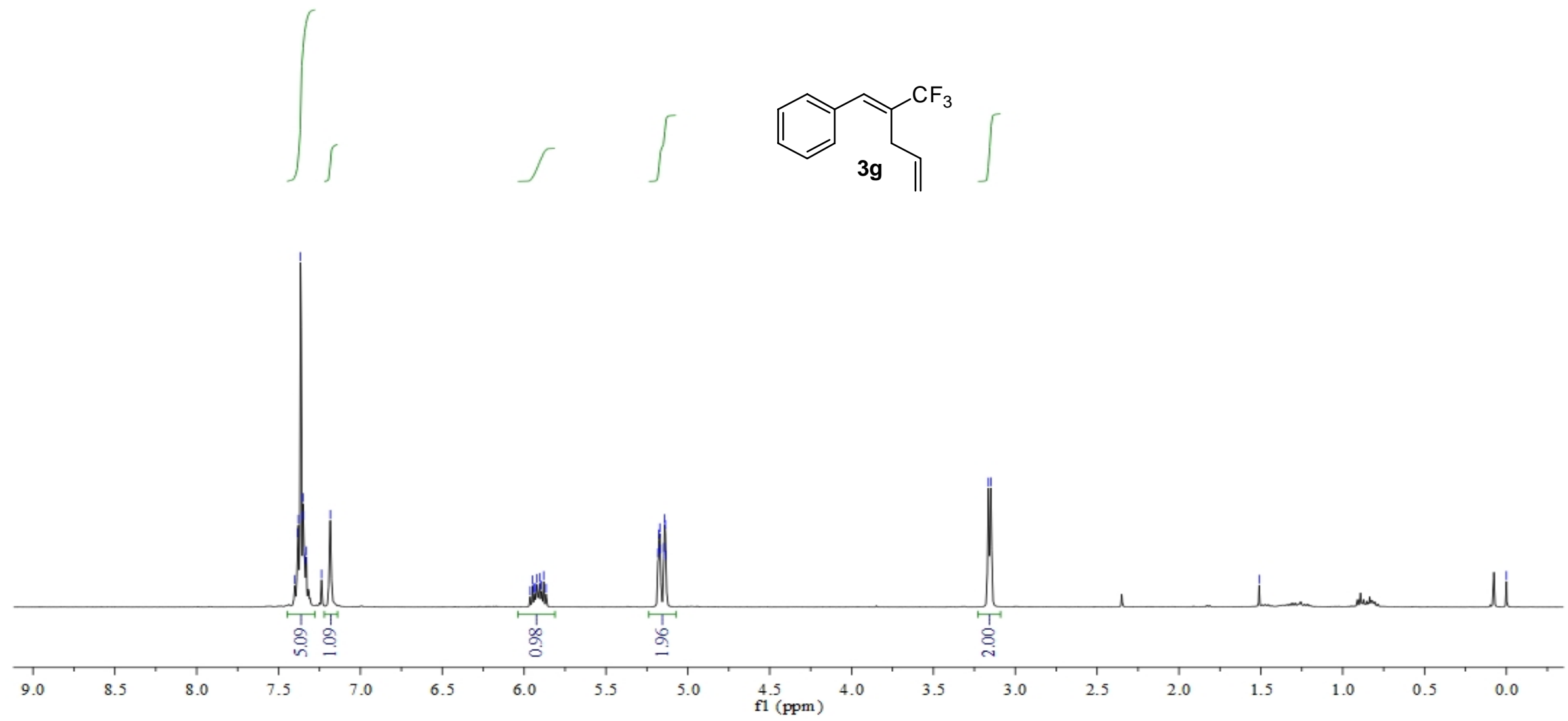


${ }^{13}$ C-NMR

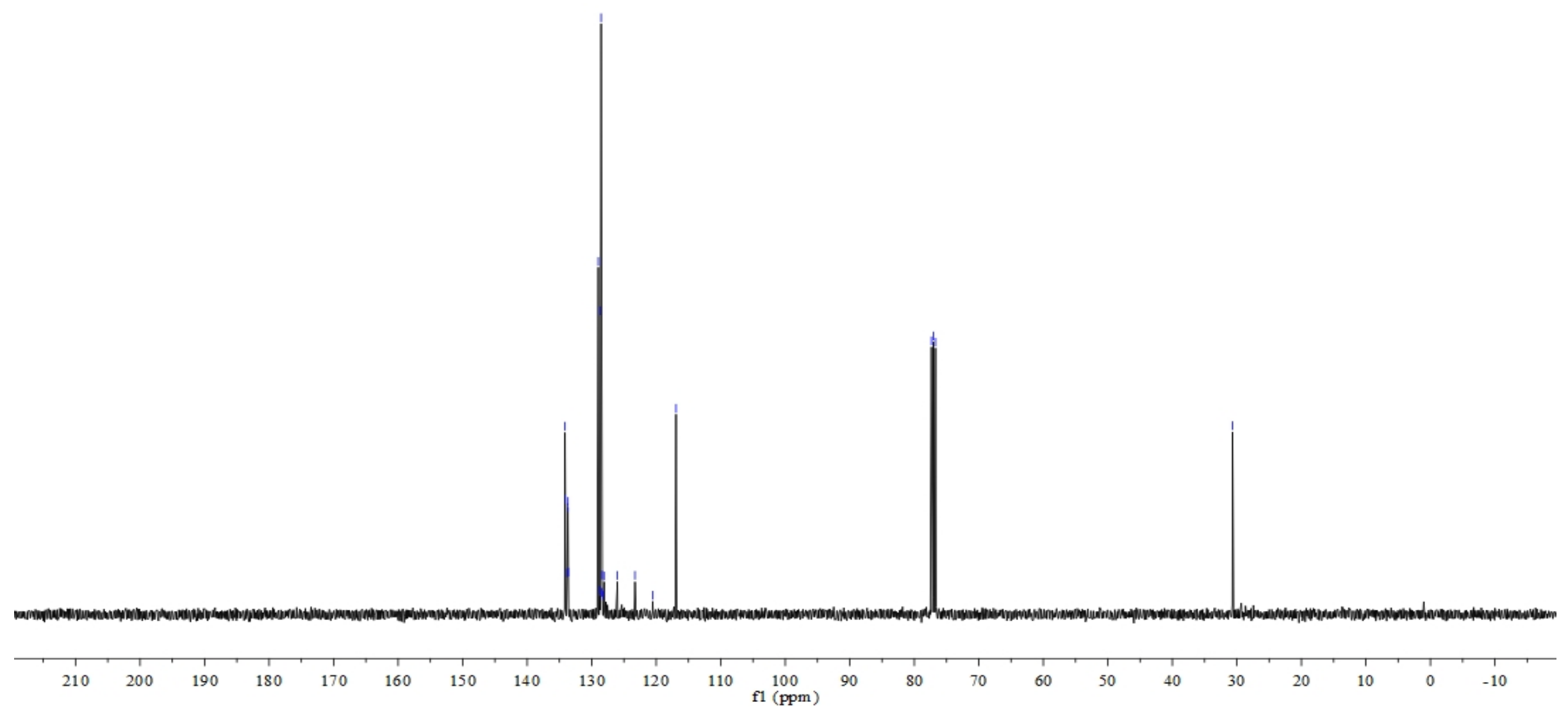


${ }^{19}$ F-NMR
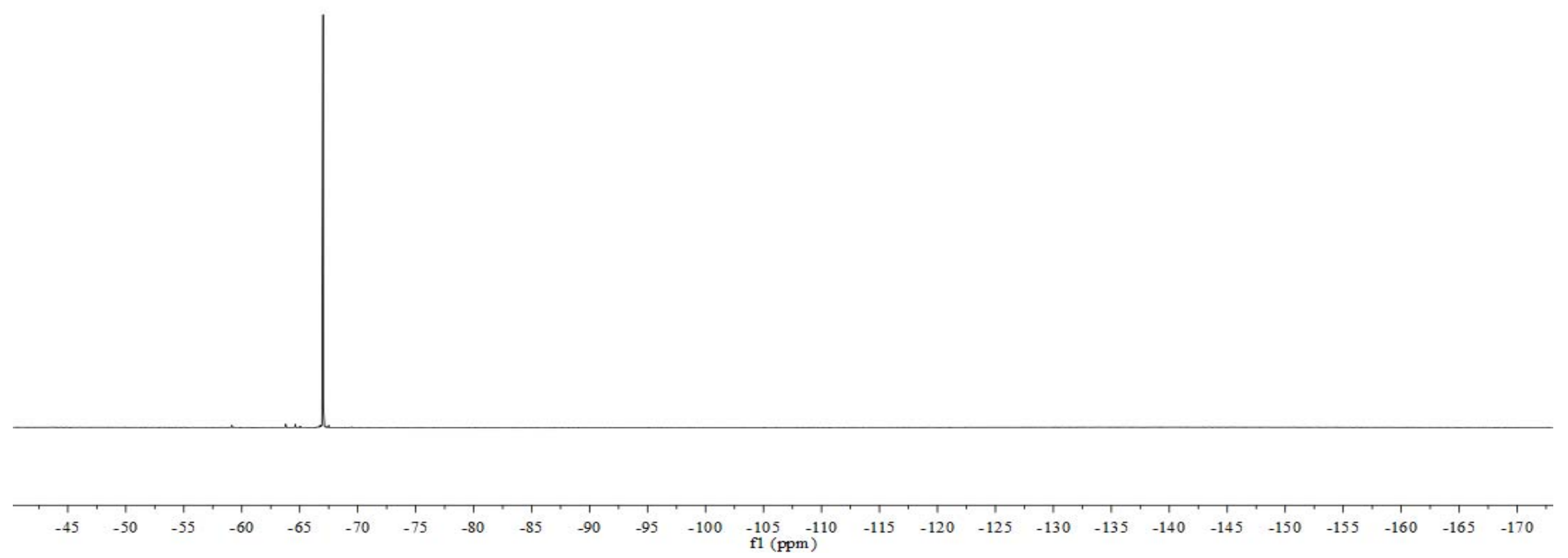


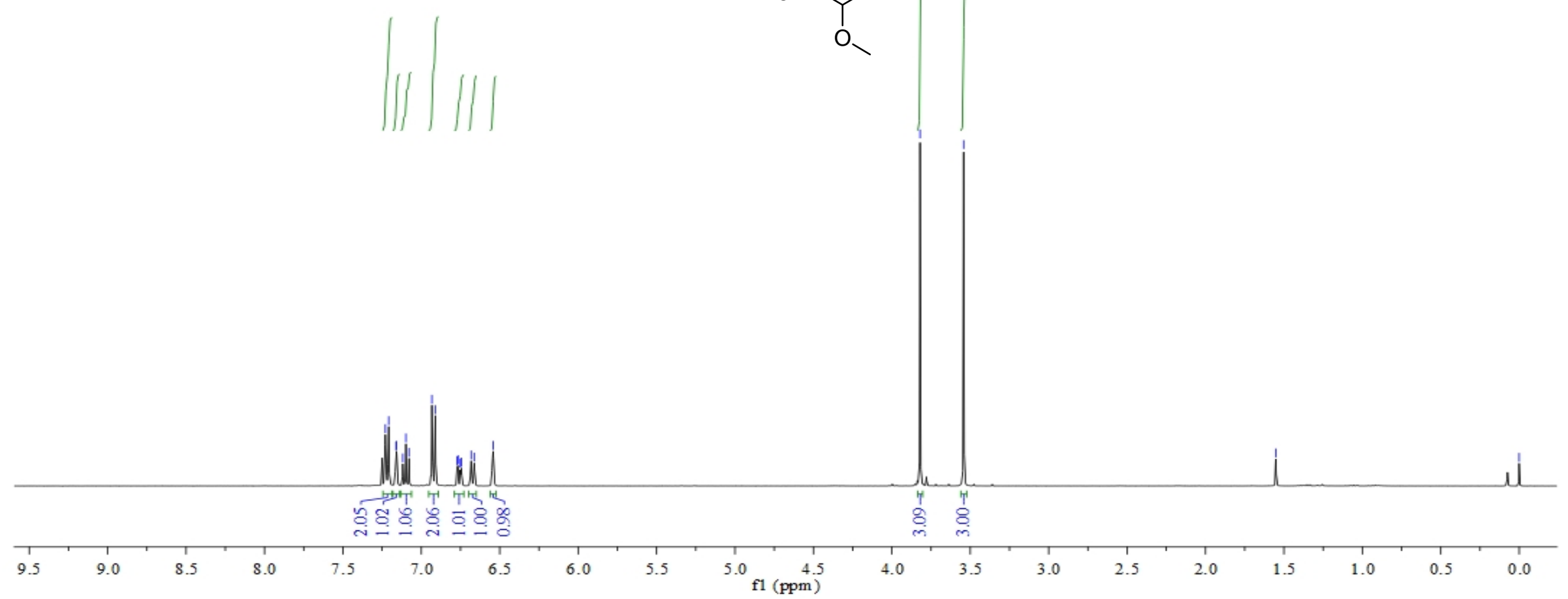


${ }^{13}$ C-NMR
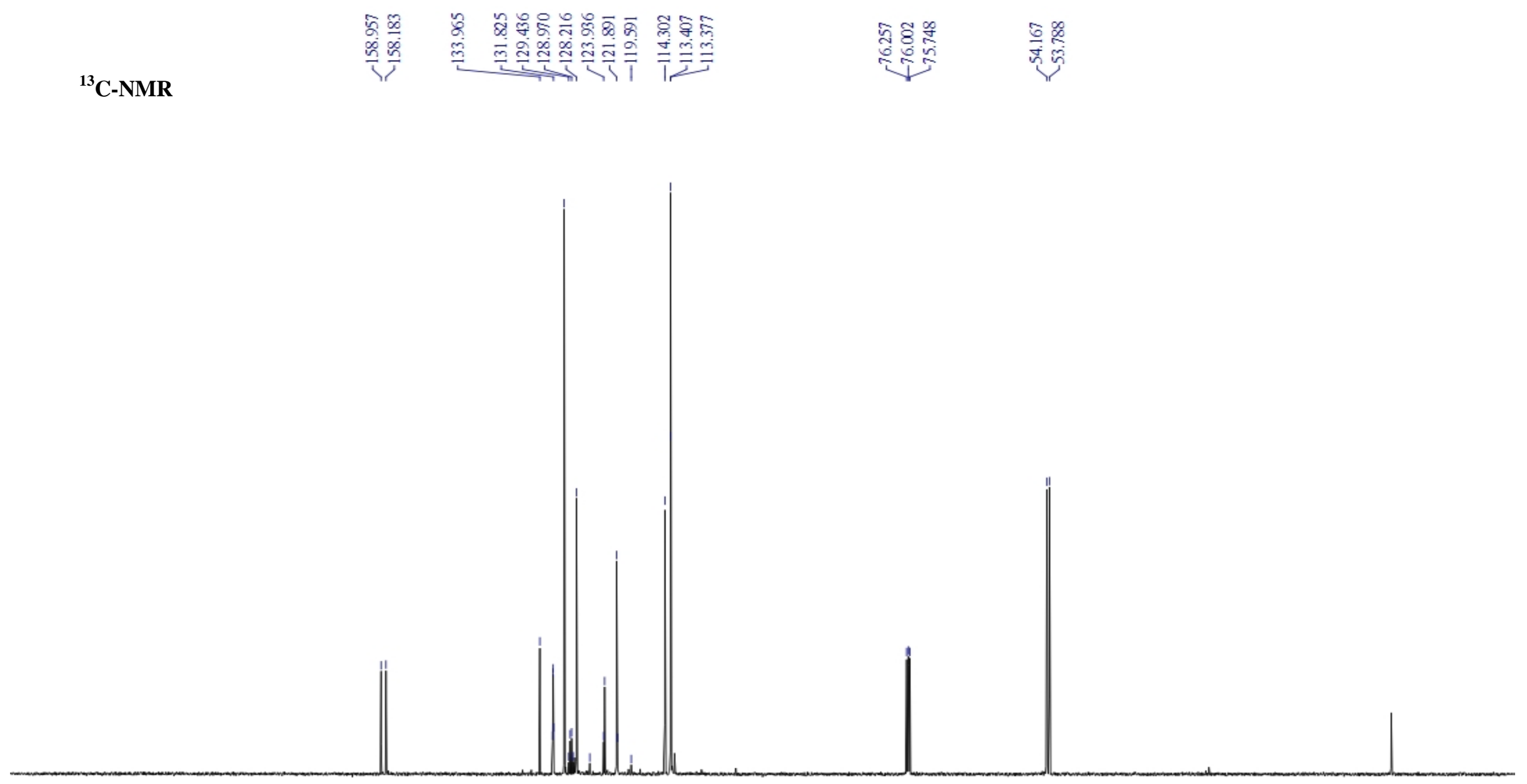
${ }^{19}$ F-NMR

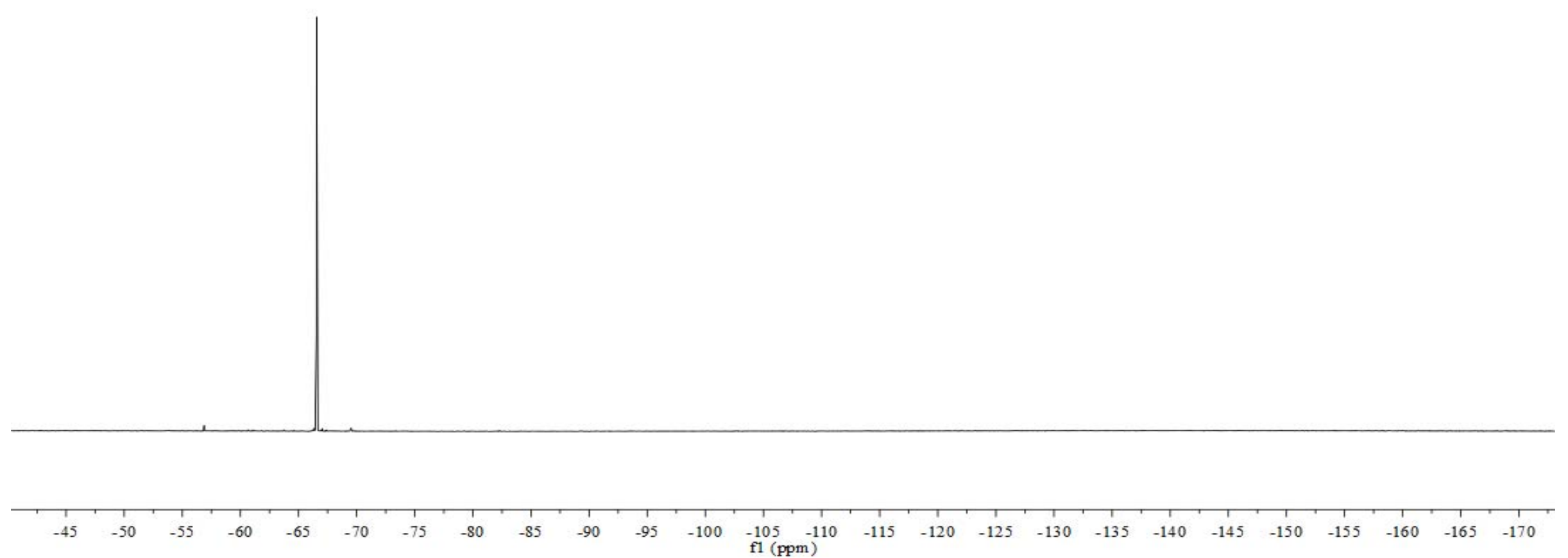


${ }^{1}$ H-NMR

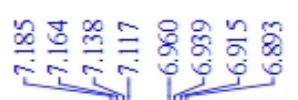
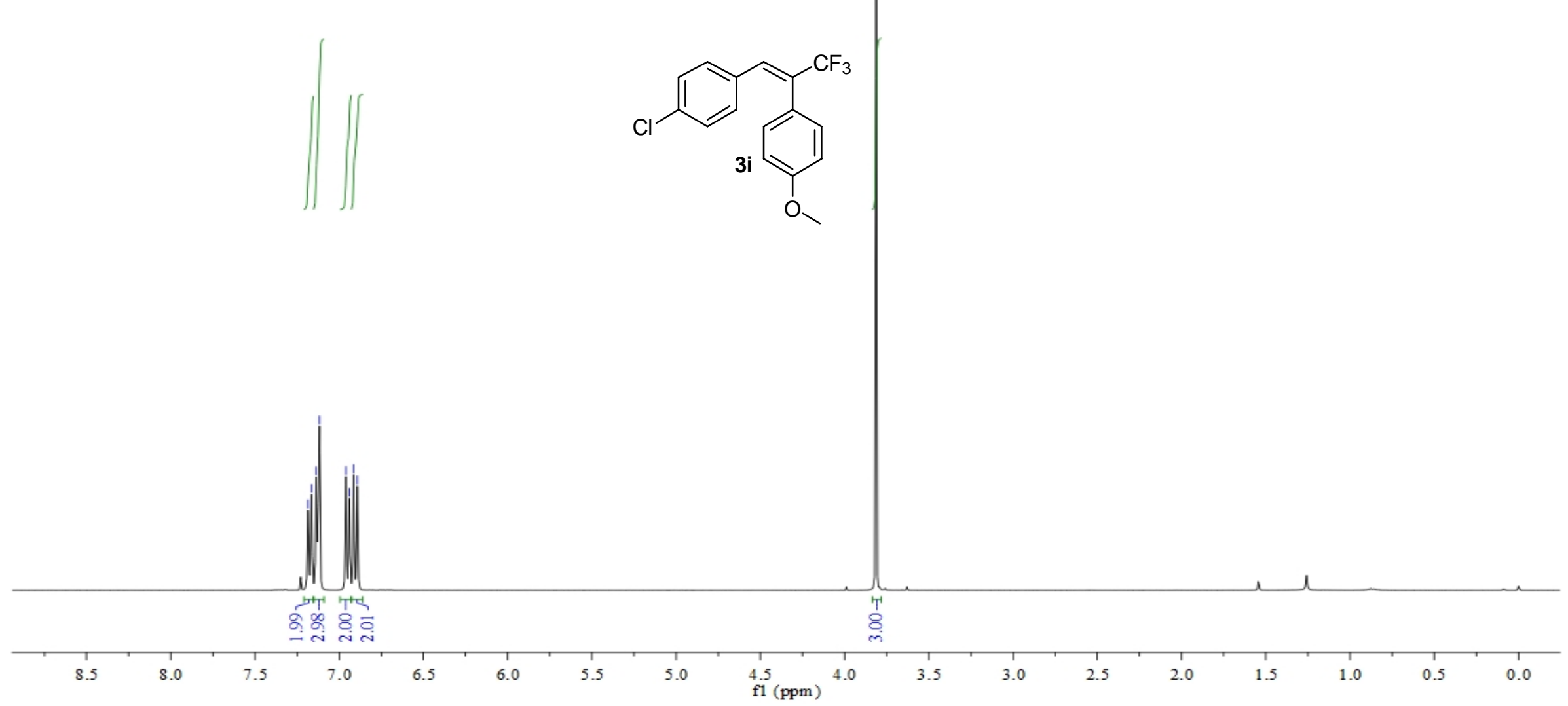
<smiles>CC(C)=C(C)C</smiles> 
${ }^{19}$ F-NMR

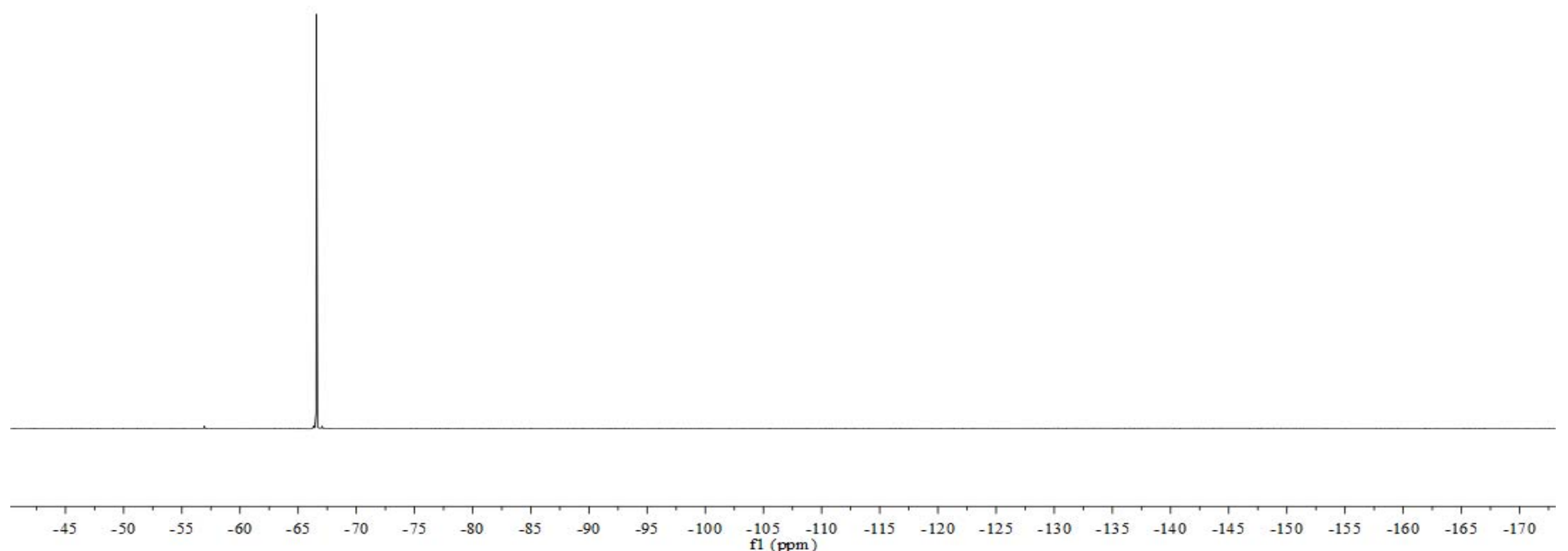


1H-NMR

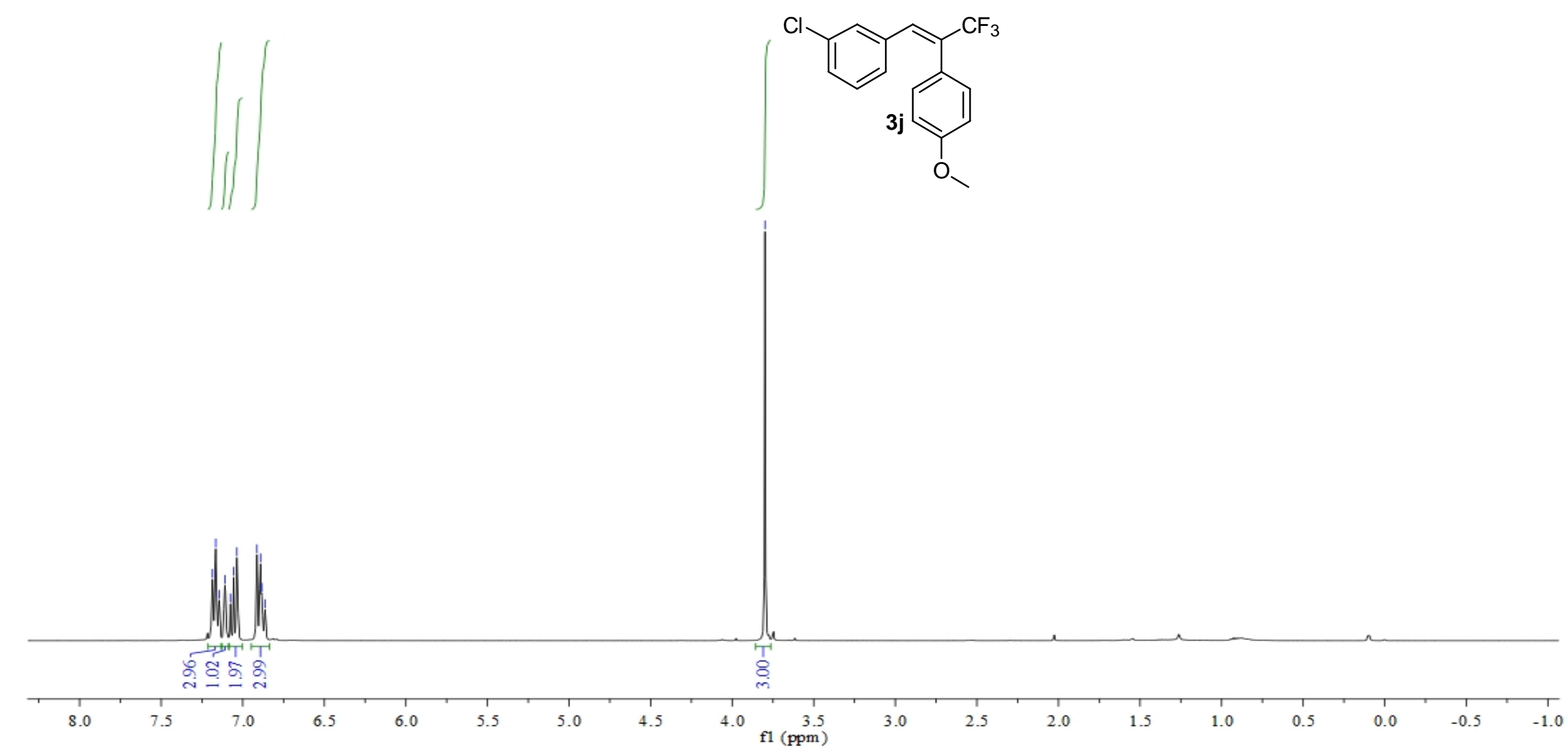




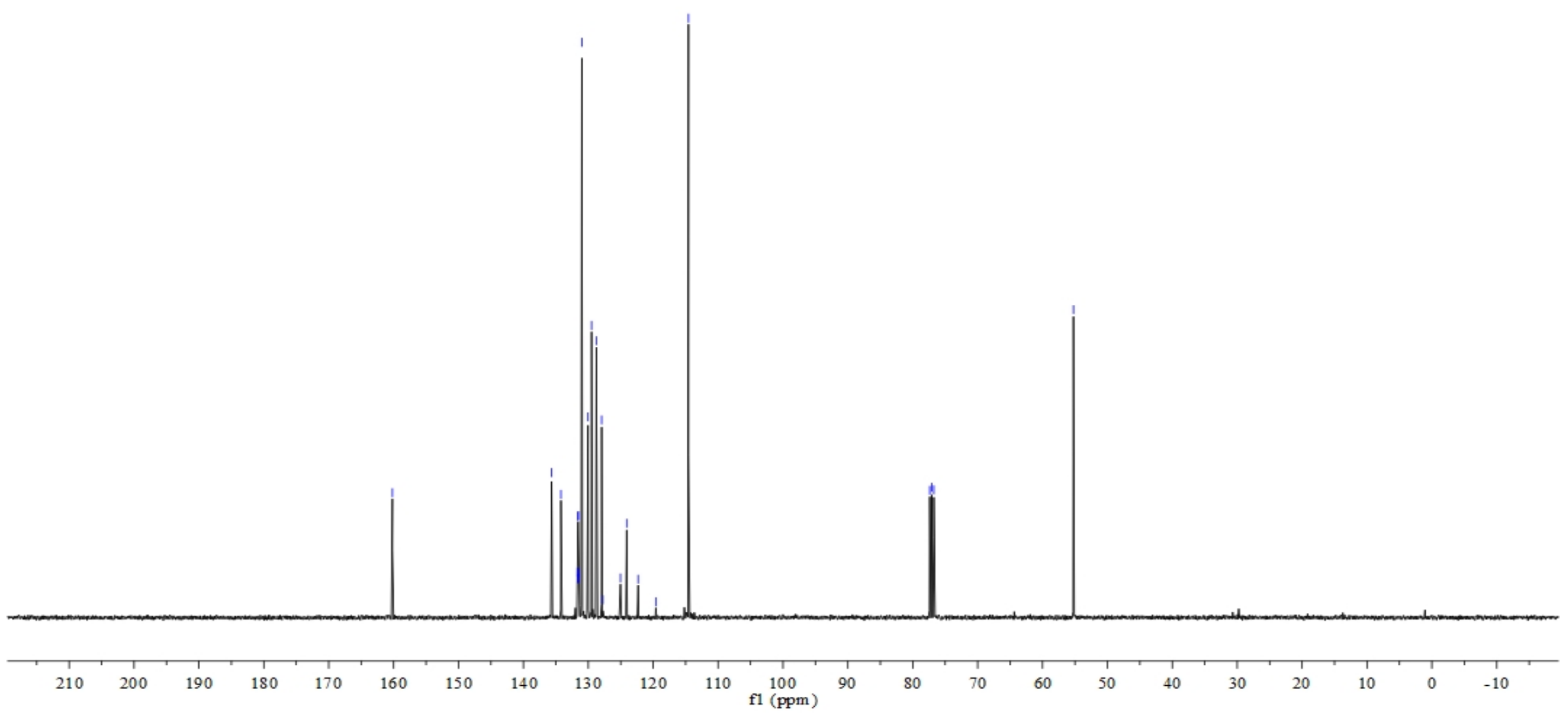


${ }^{19}$ F-NMR

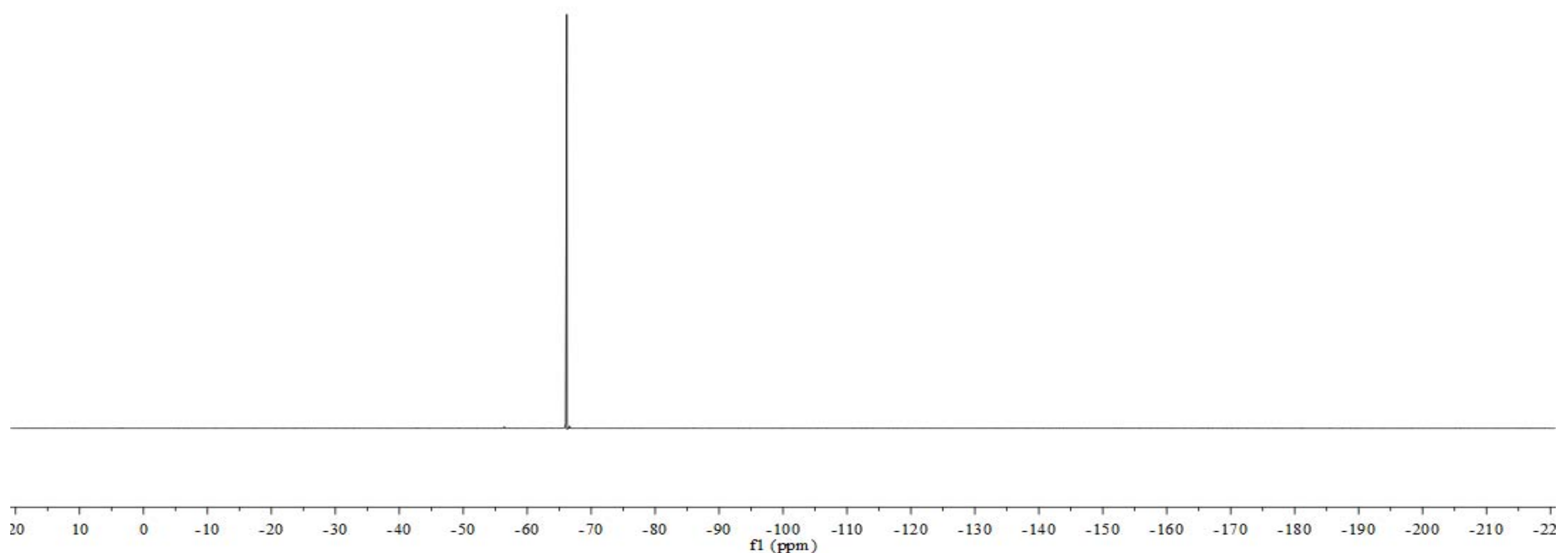


${ }^{1}$ H-NMR

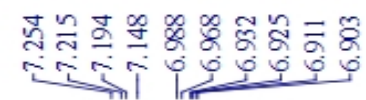

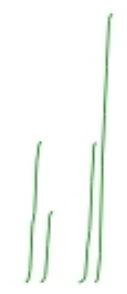

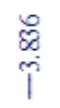

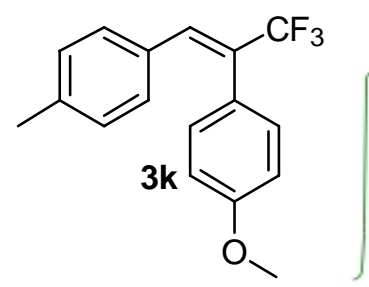

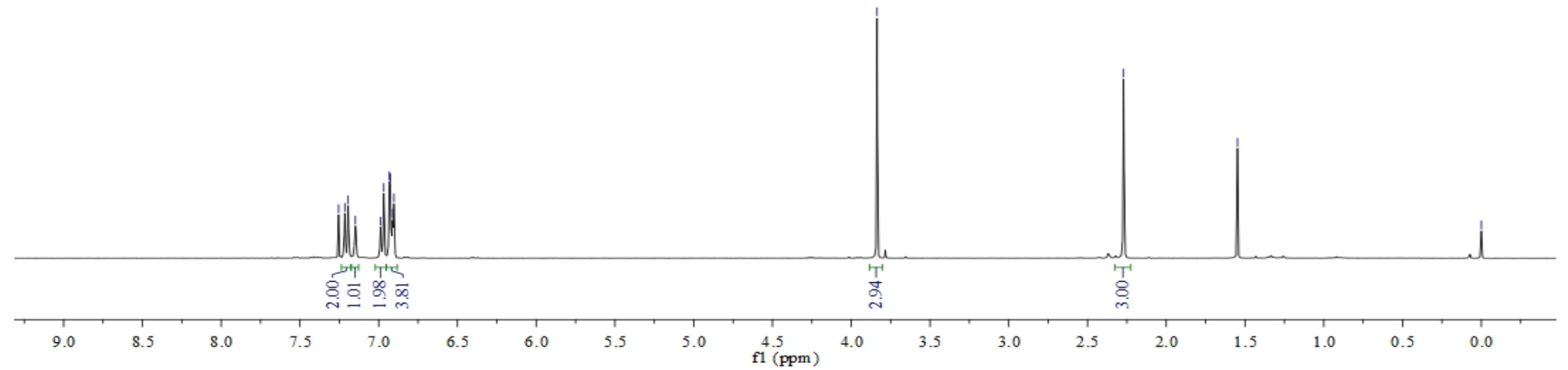




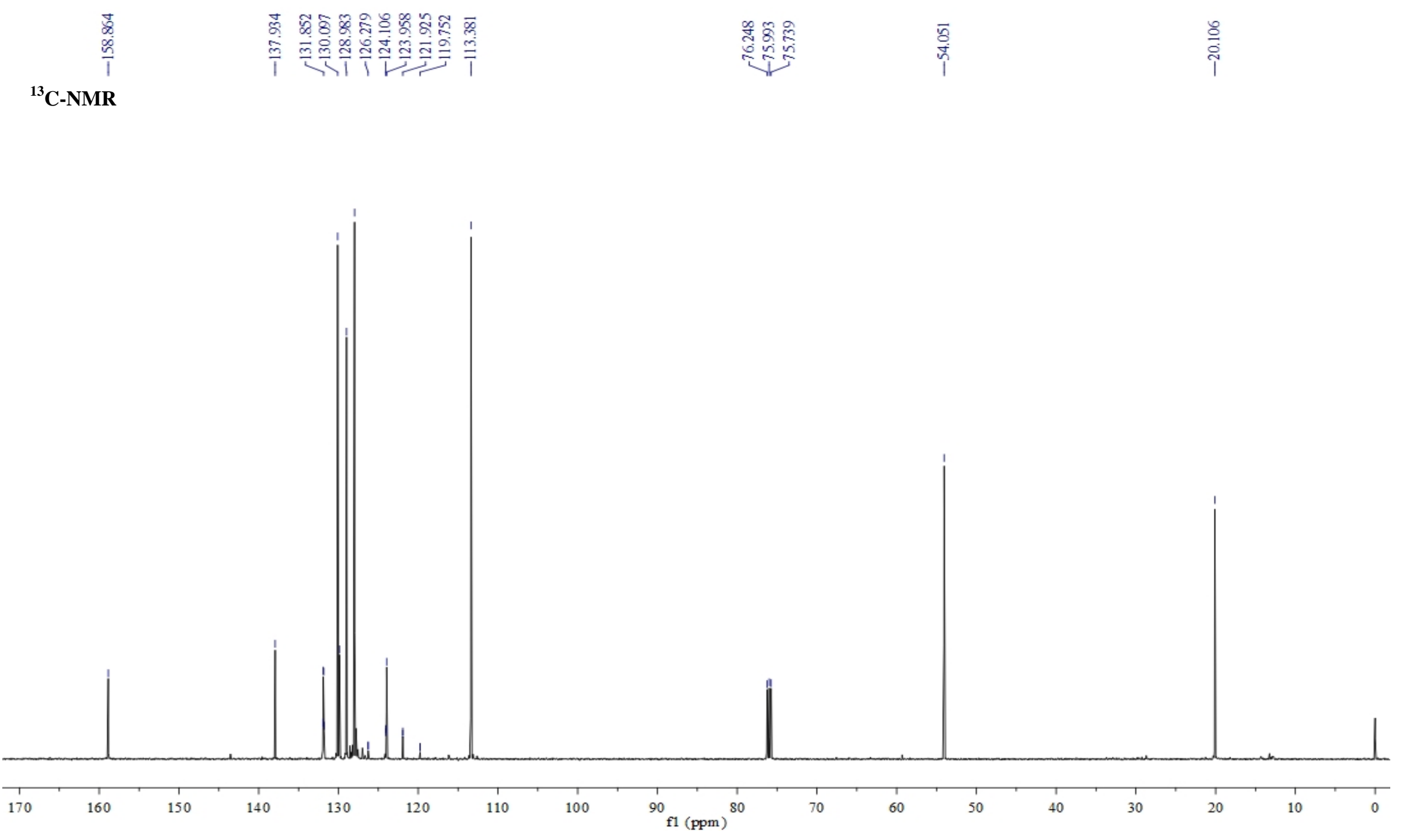


${ }^{19}$ F-NMR

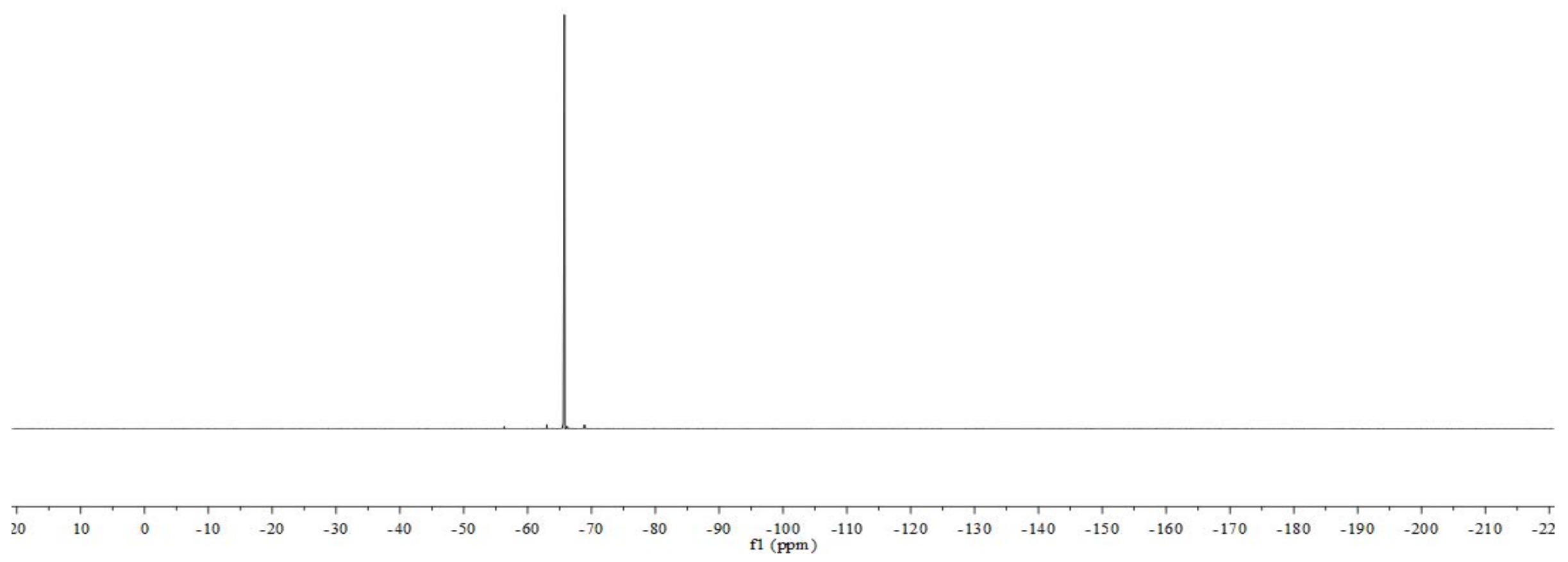



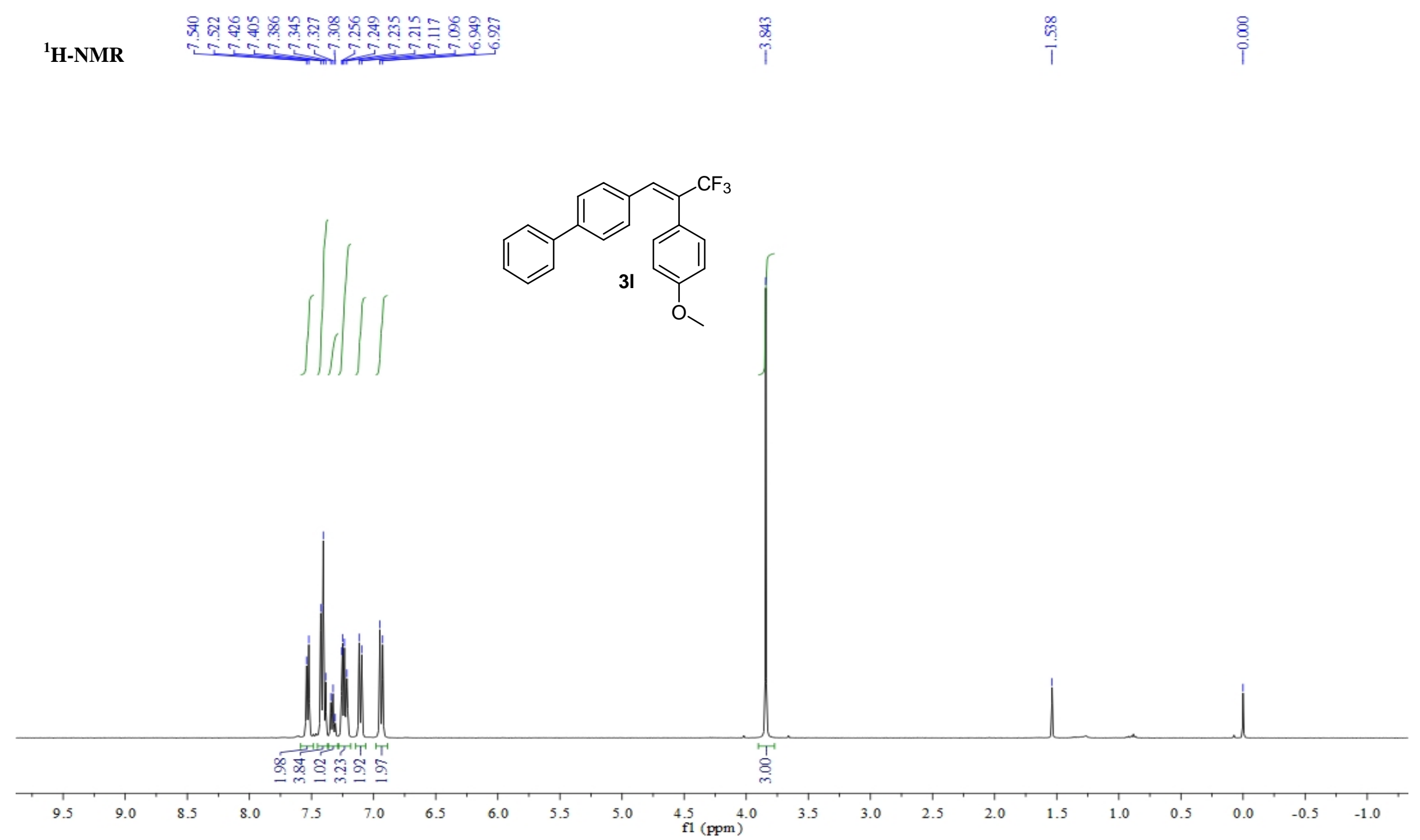


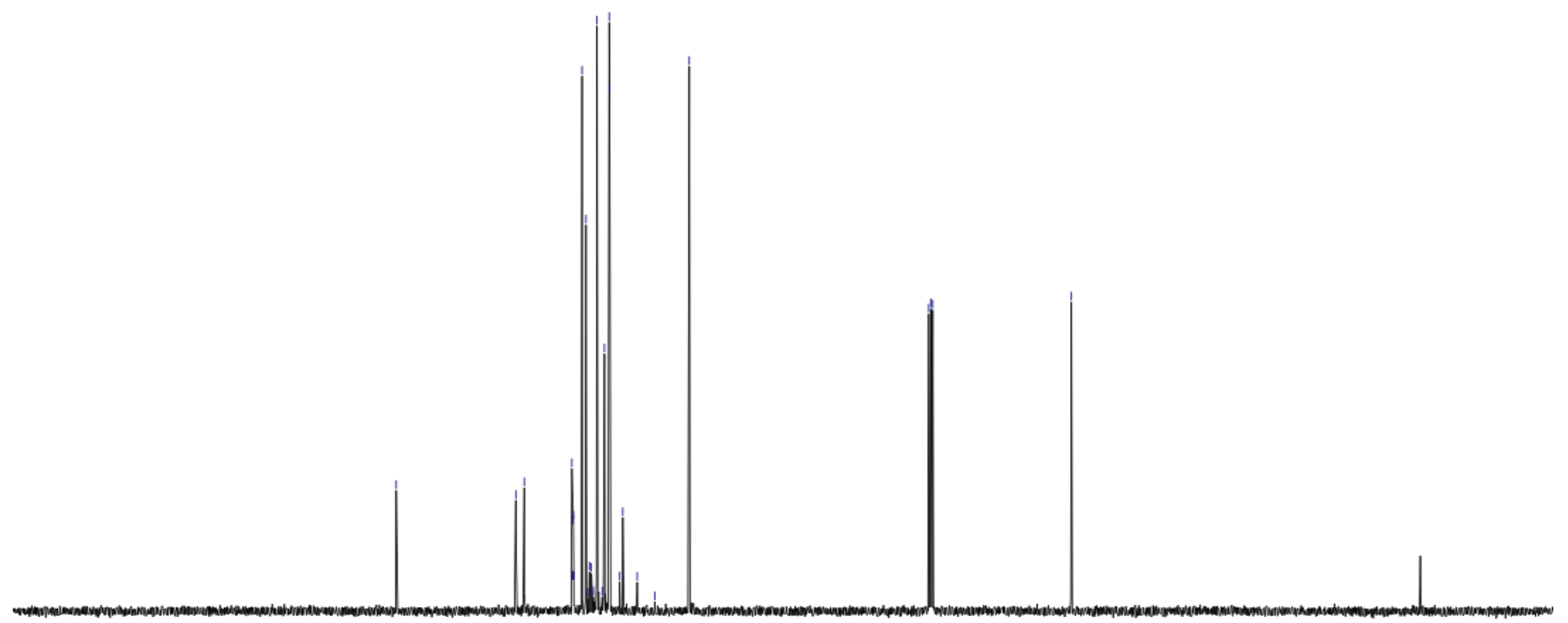


${ }^{19}$ F-NMR

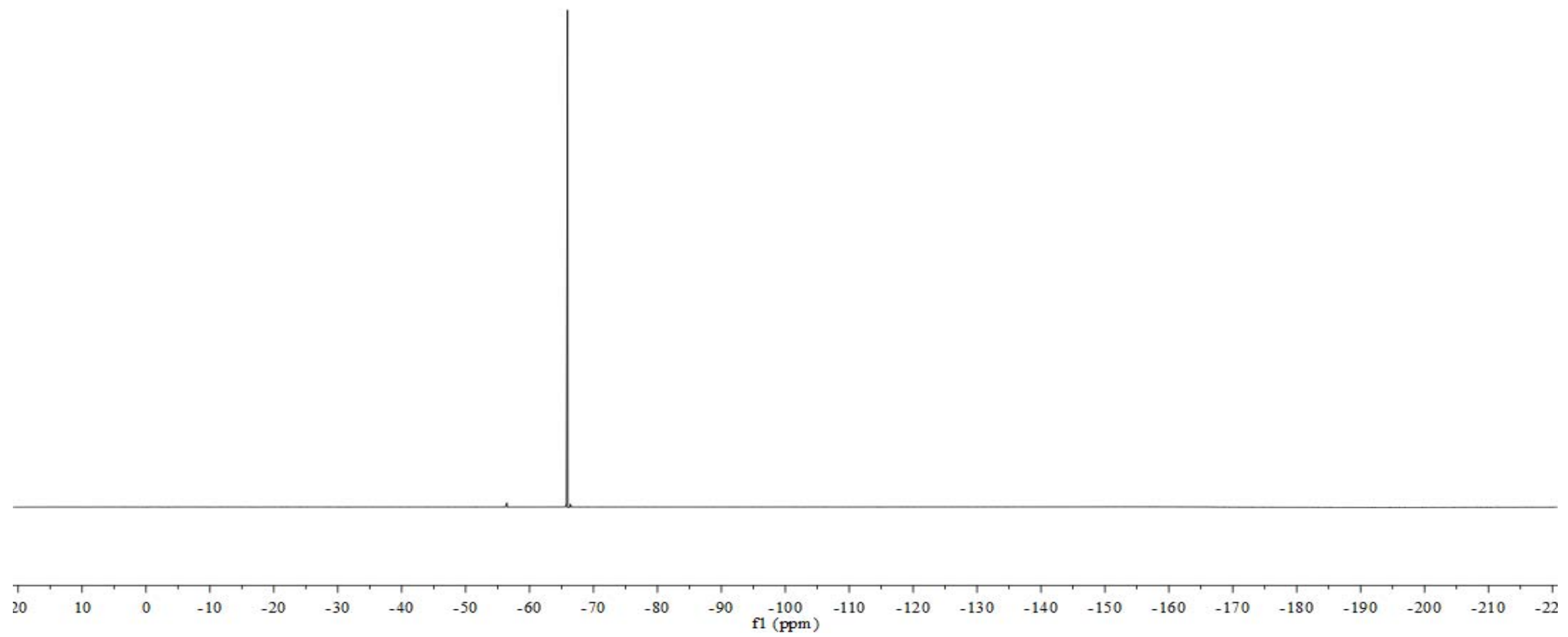




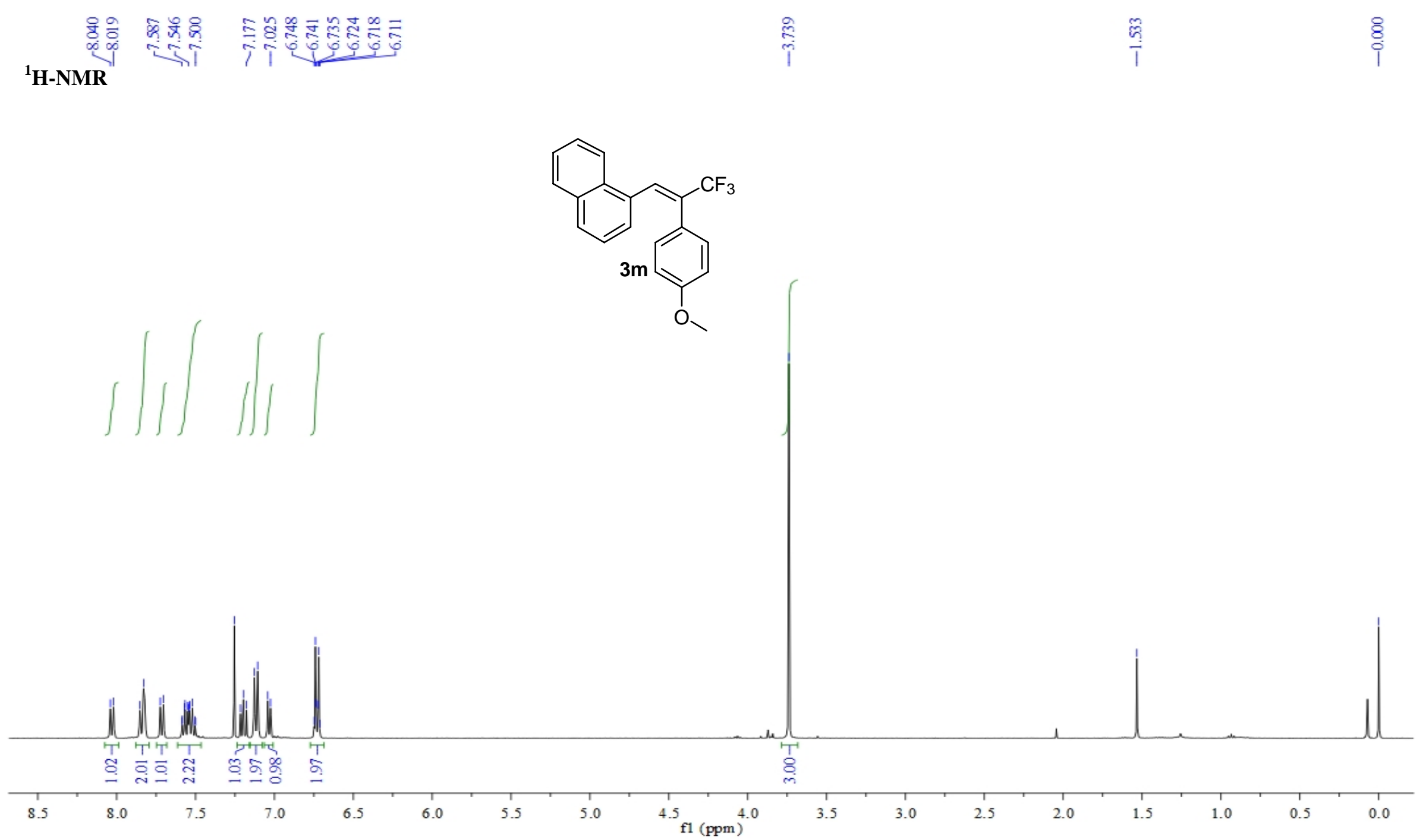


${ }^{13}$ C-NMR

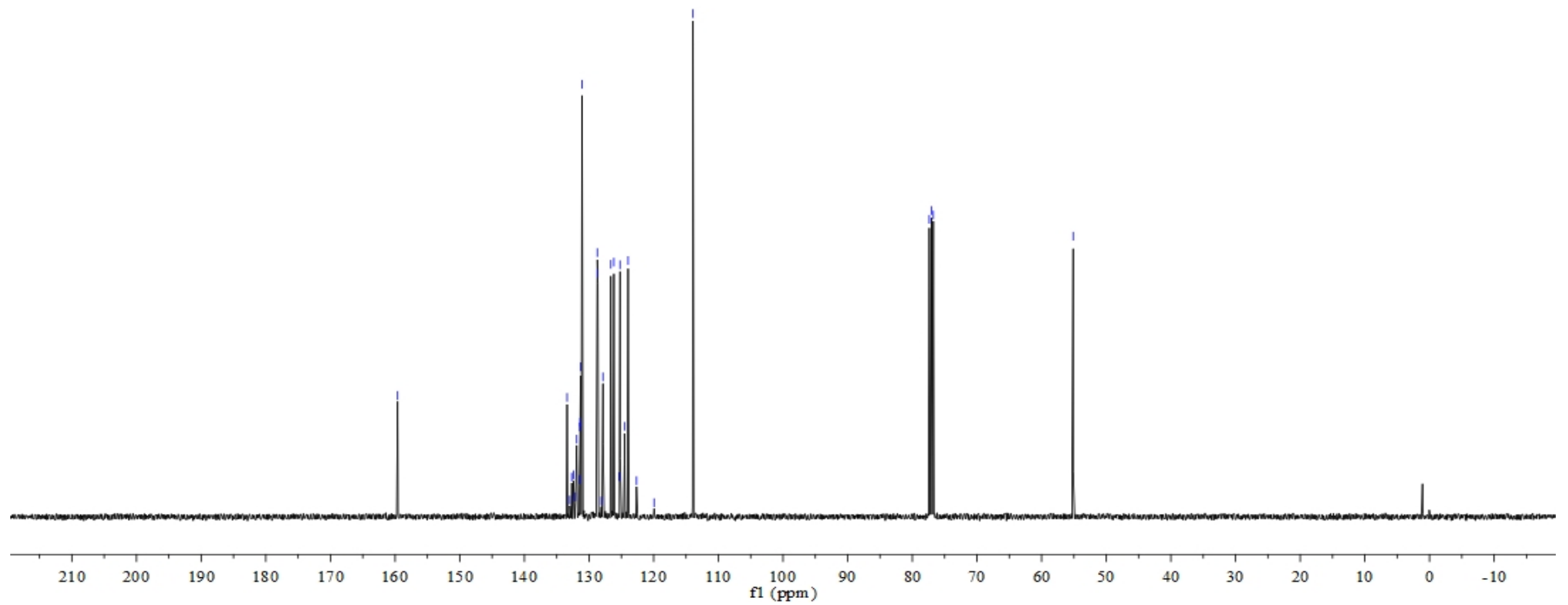


${ }^{19}$ F-NMR

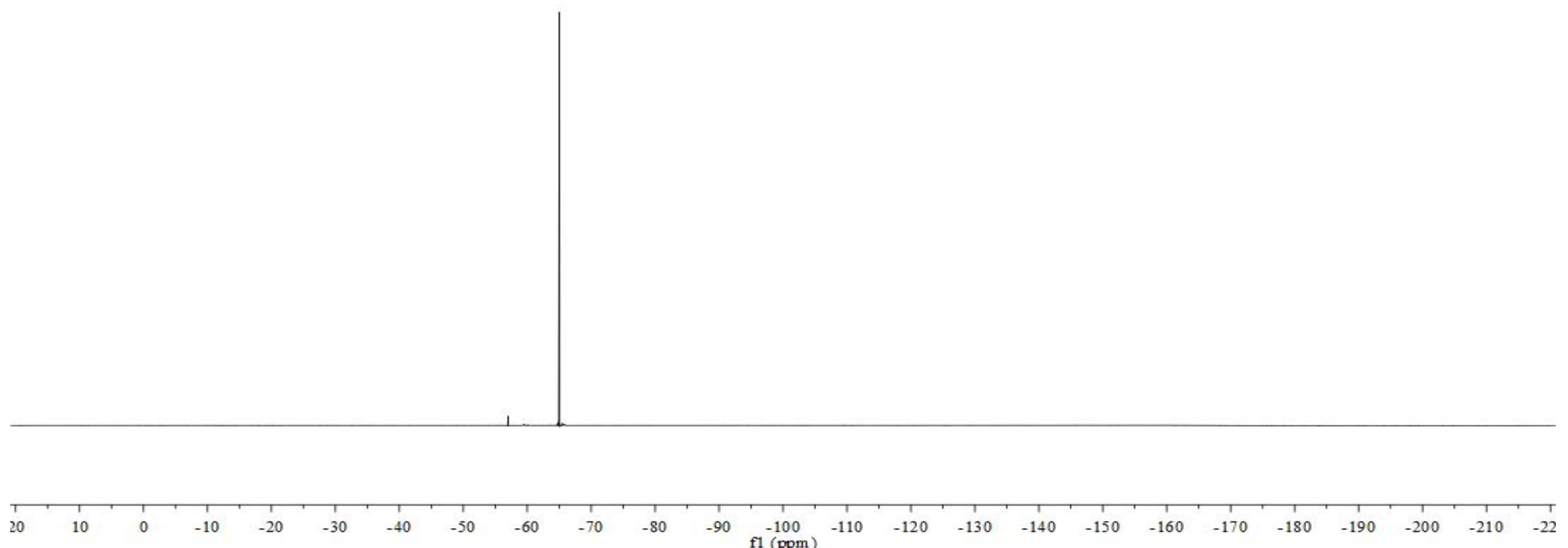




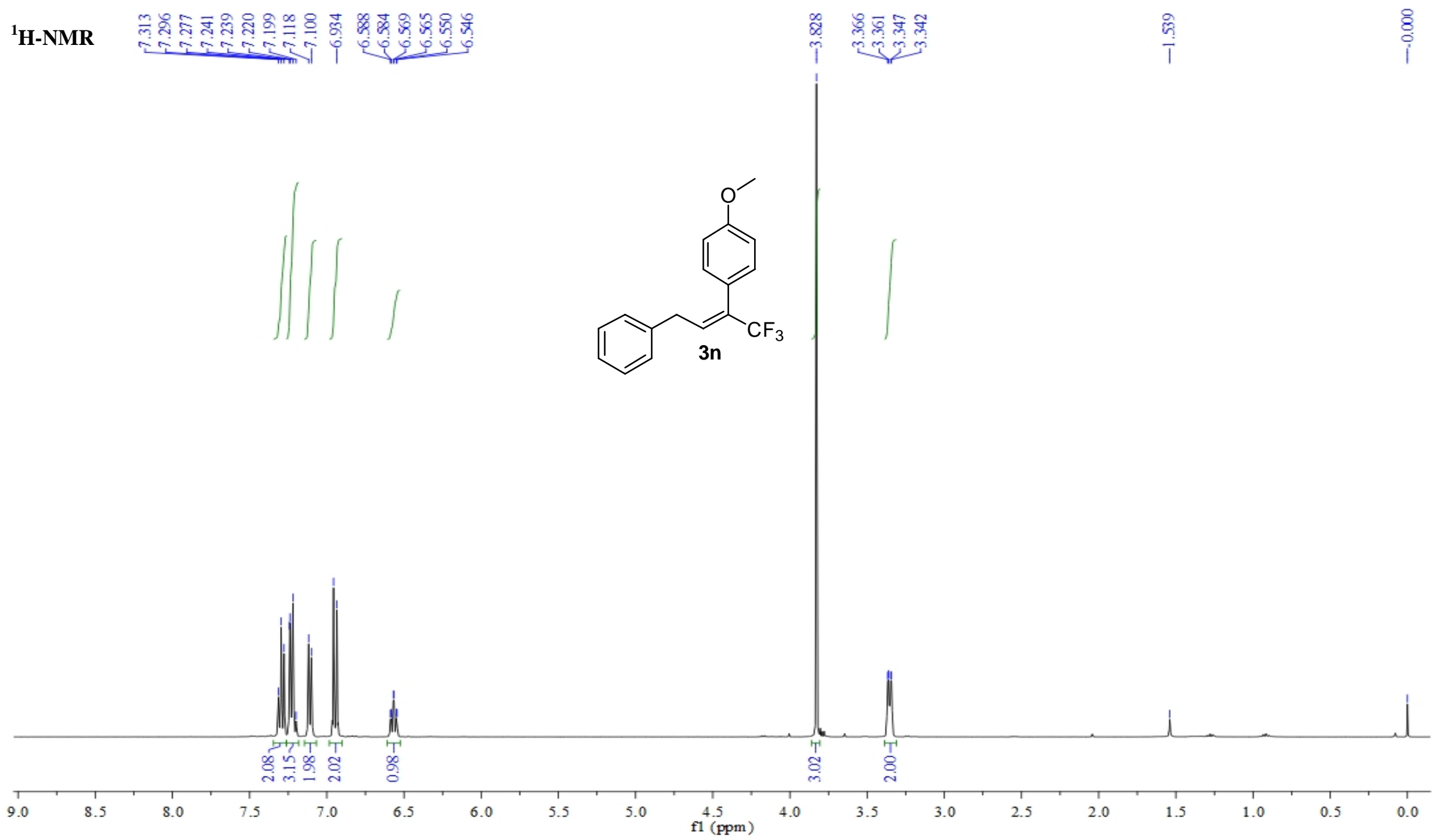




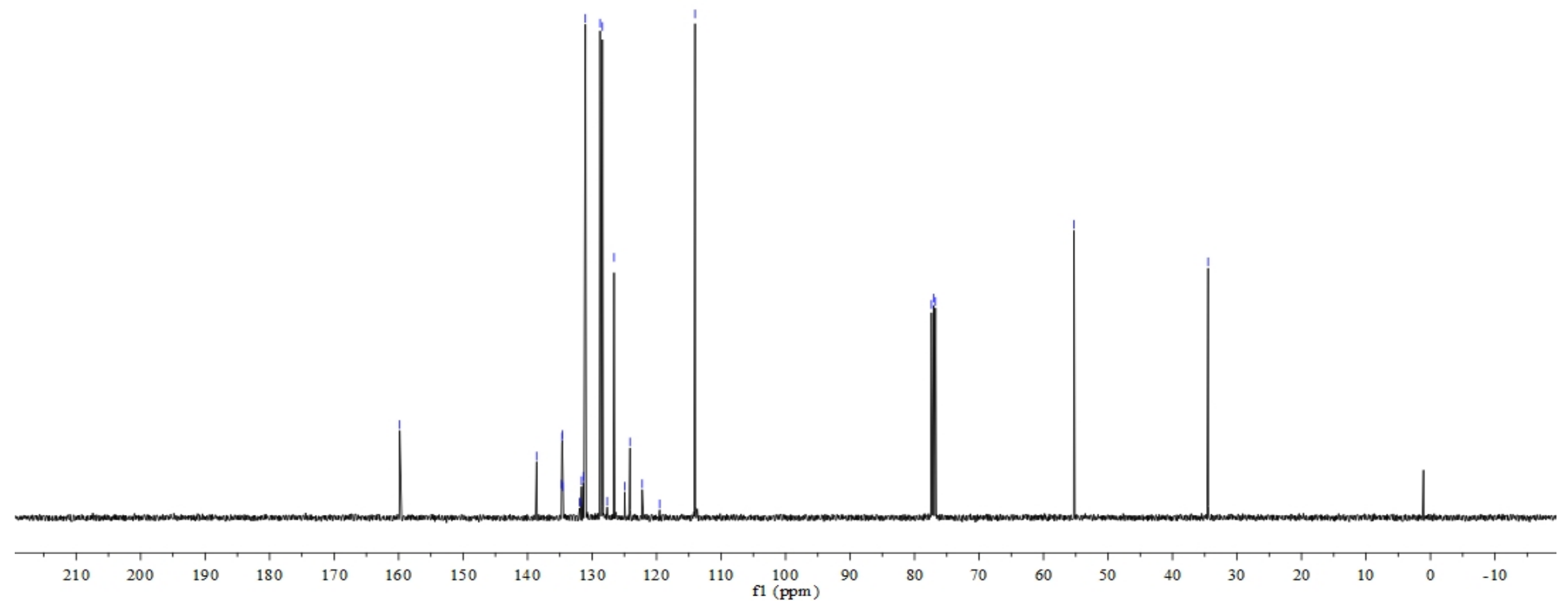


${ }^{19}$ F-NMR

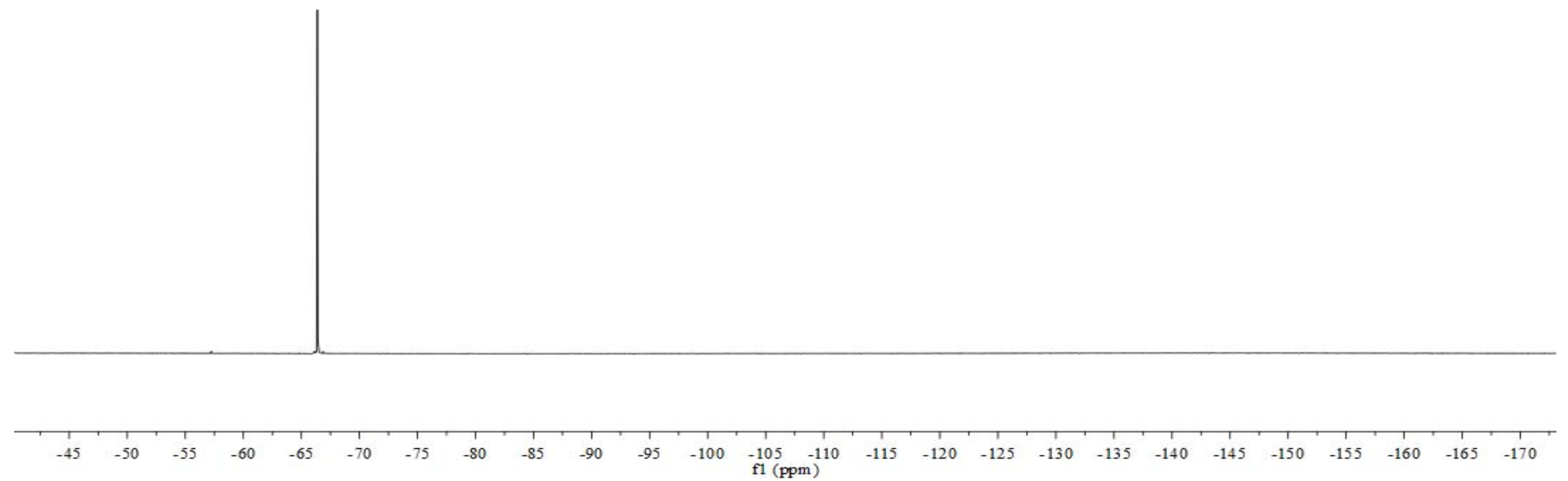




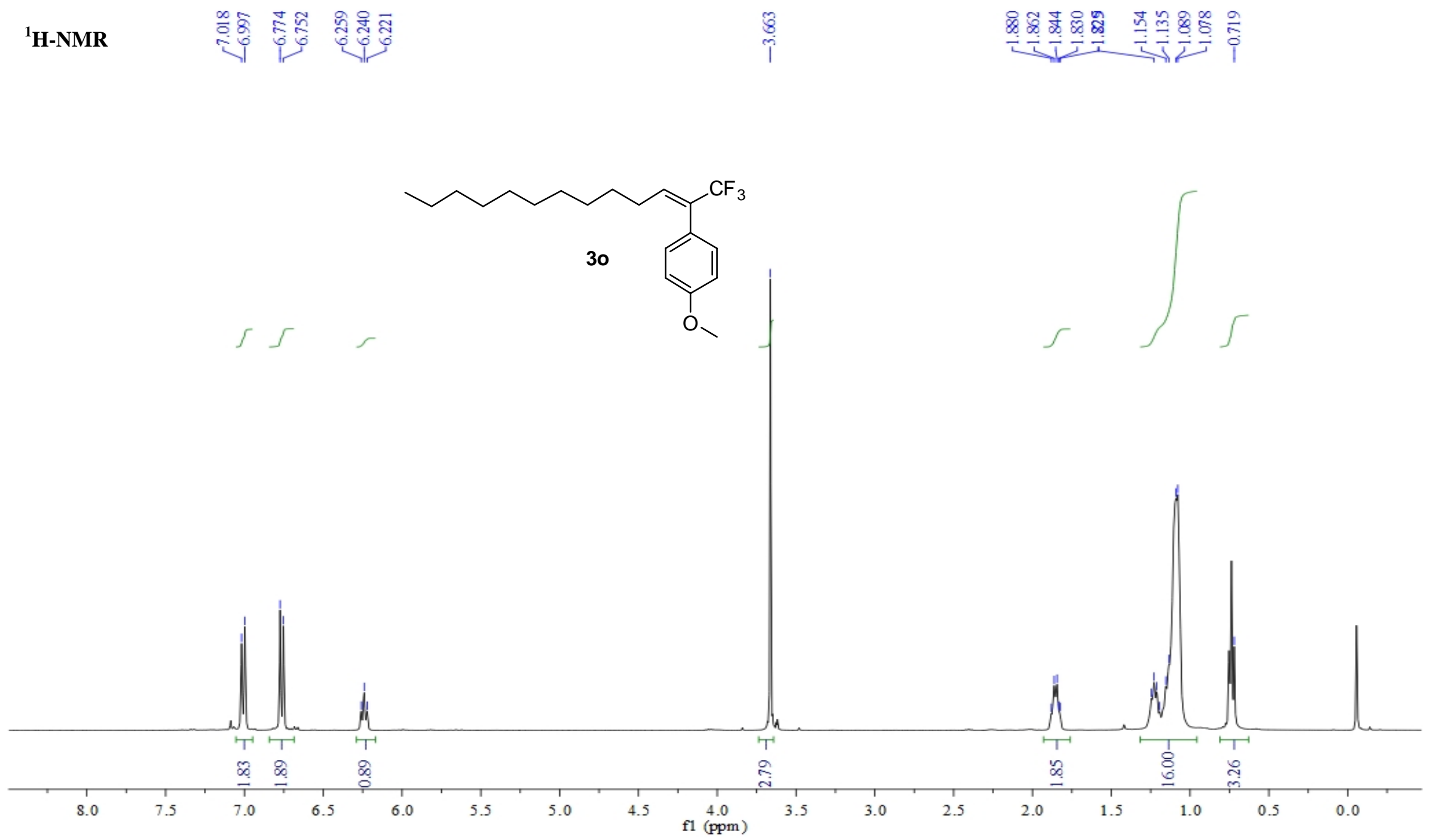




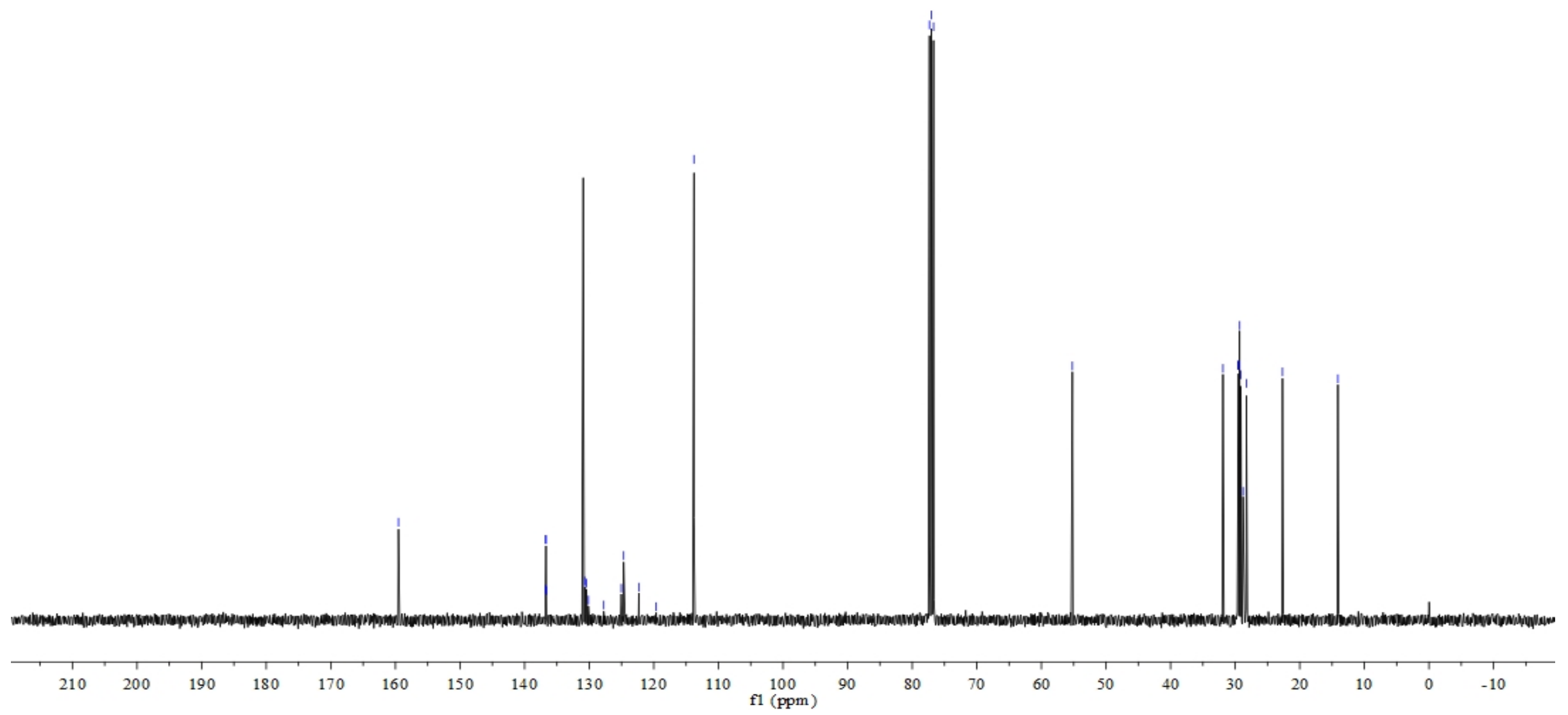


${ }^{19}$ F-NMR

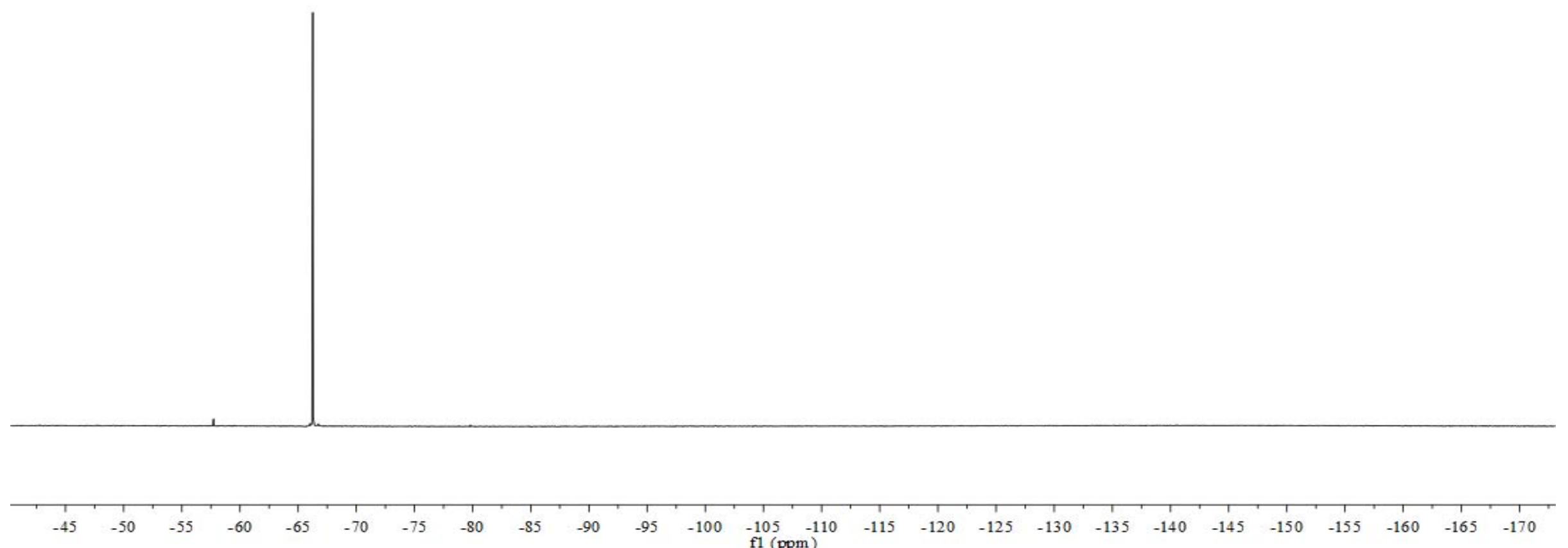


${ }^{1}$ H-NMR

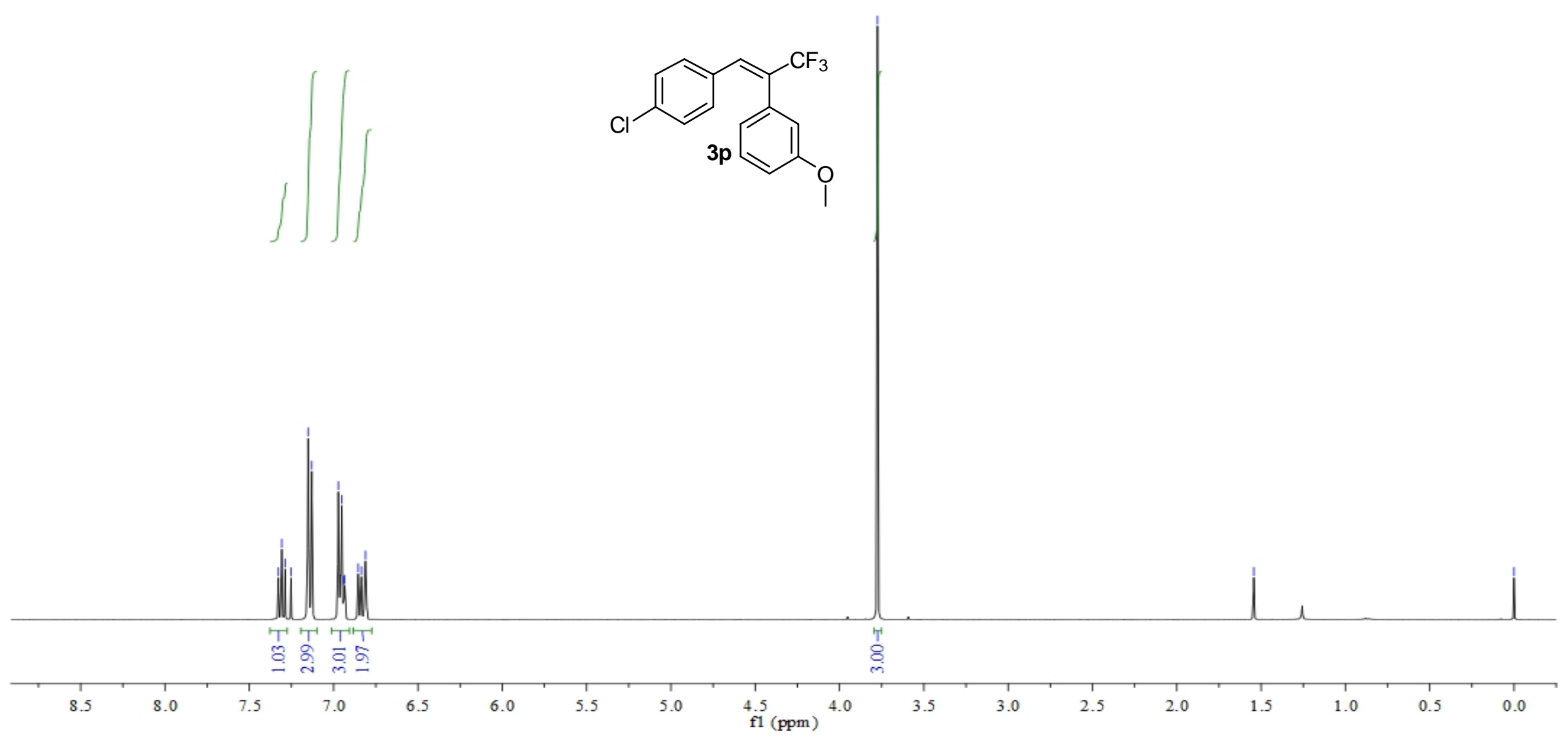


${ }^{13}$ C-NMR

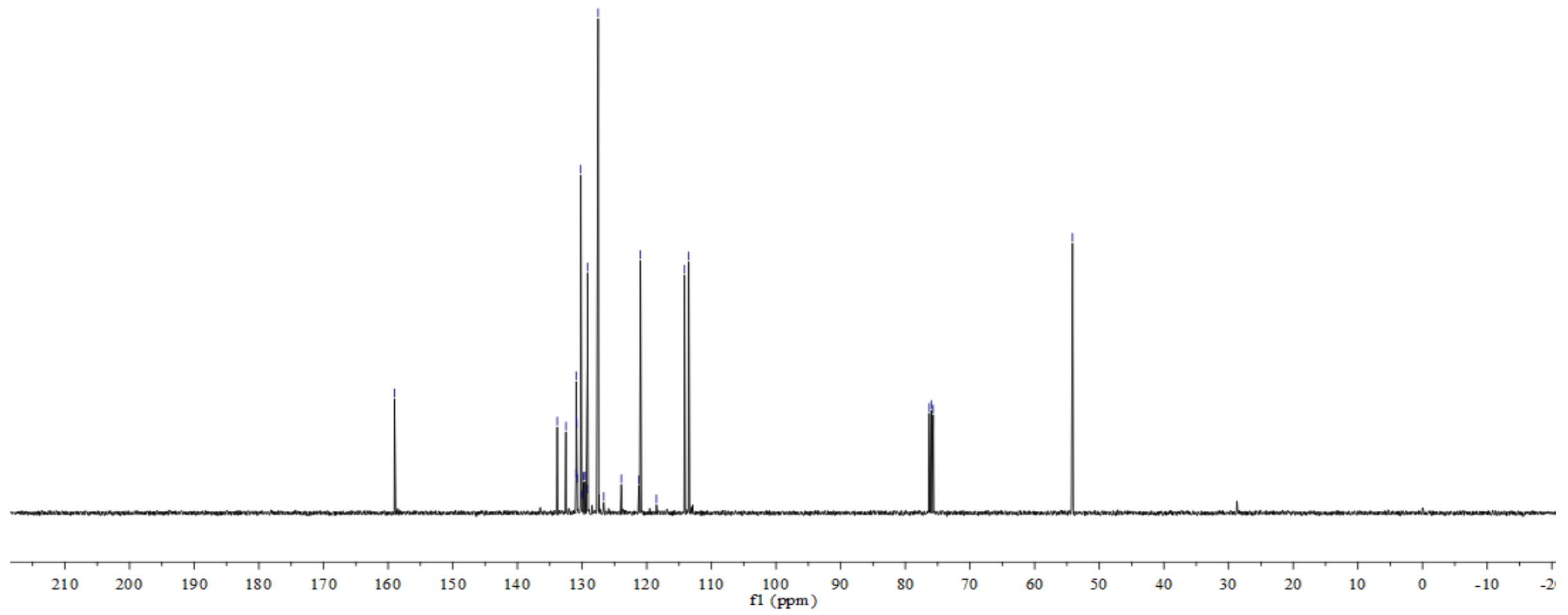


${ }^{19}$ F-NMR

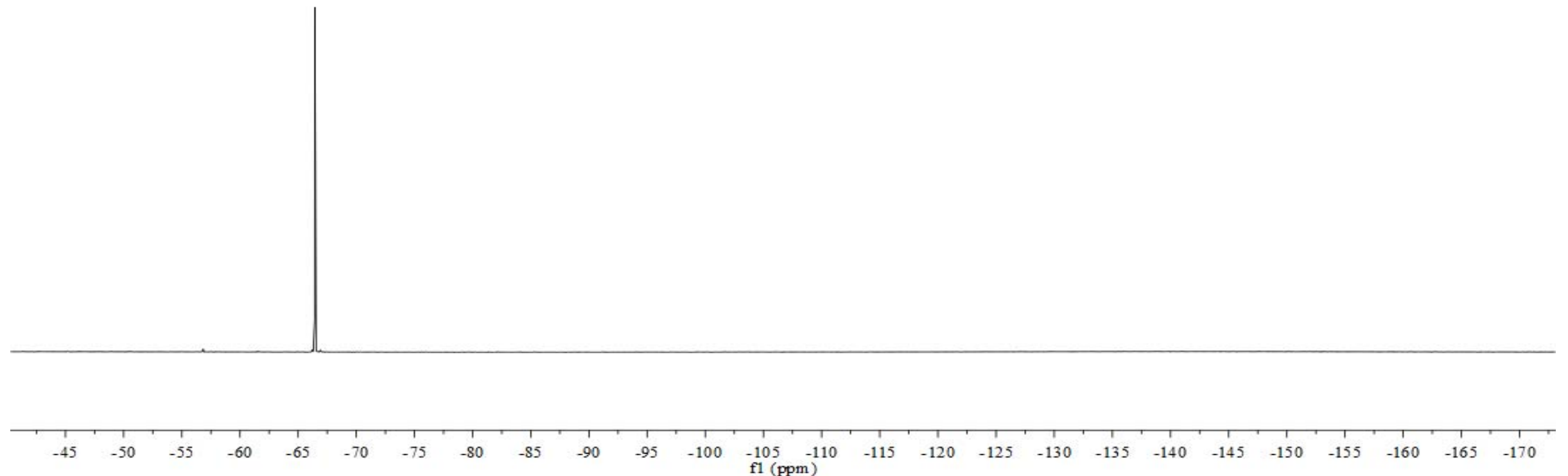




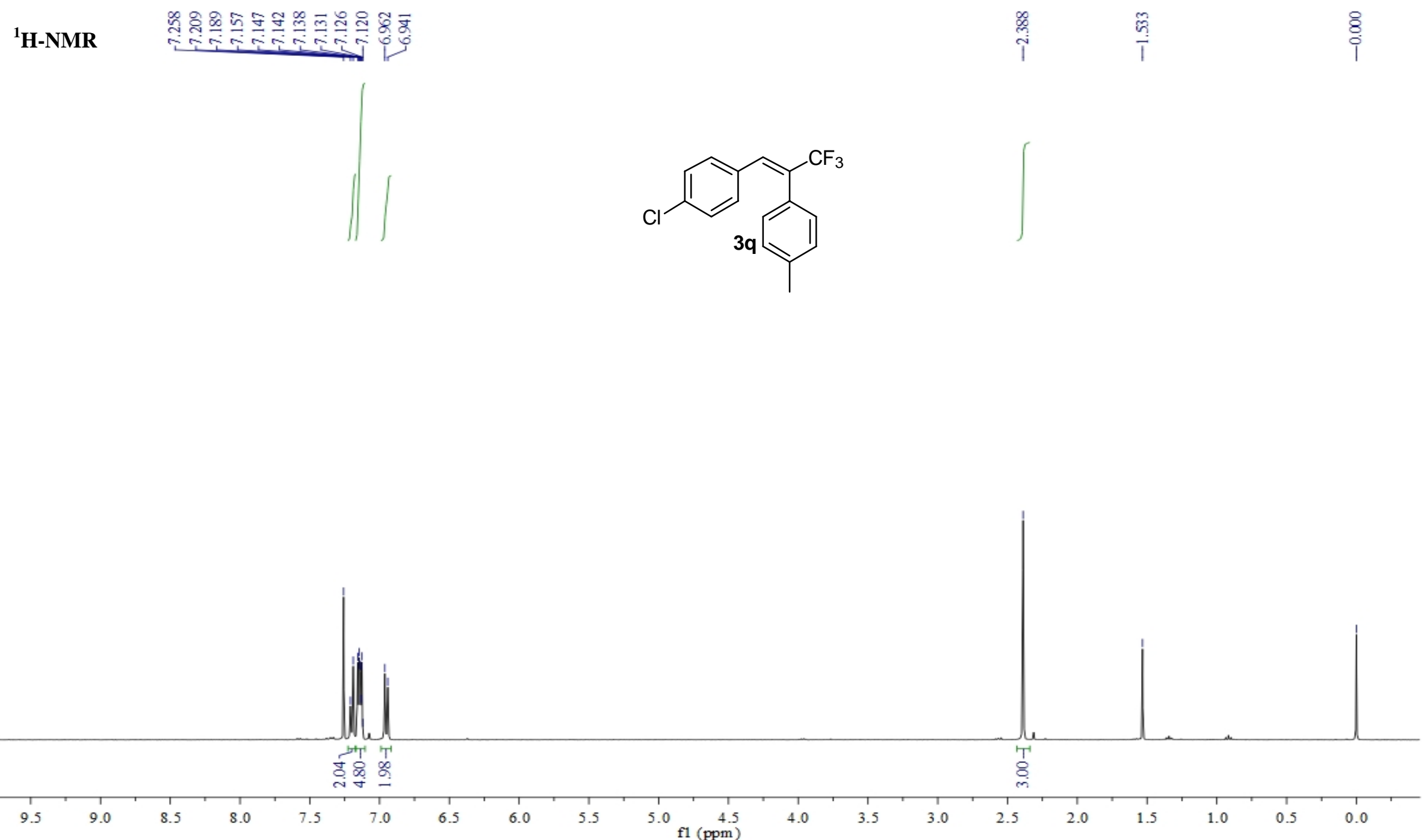




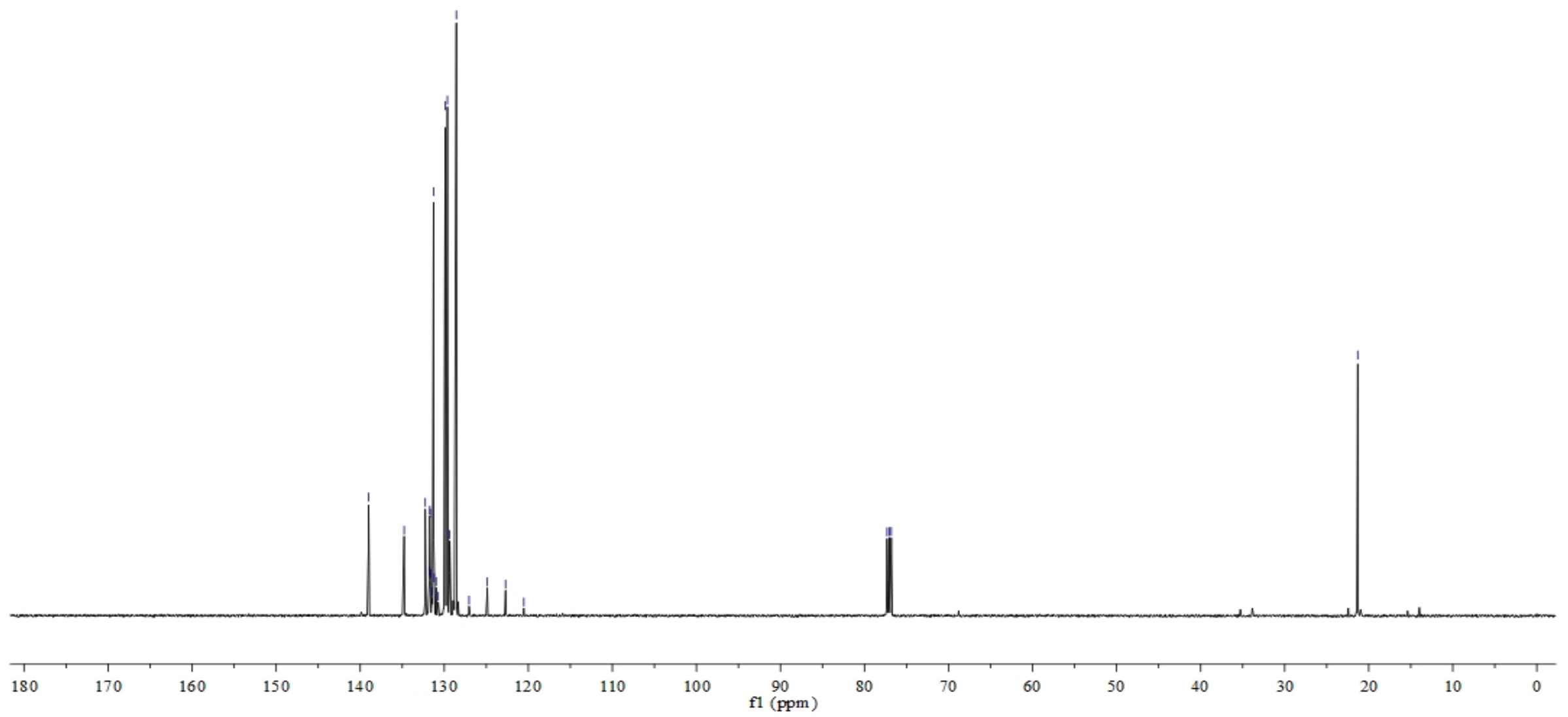


${ }^{19}$ F-NMR

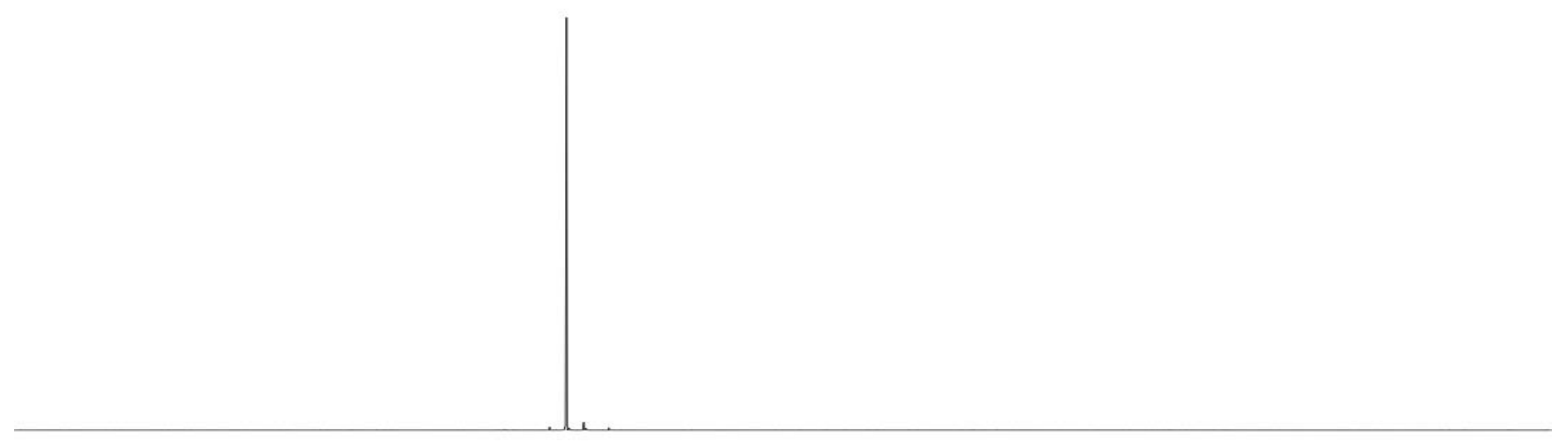

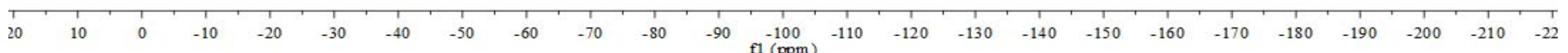




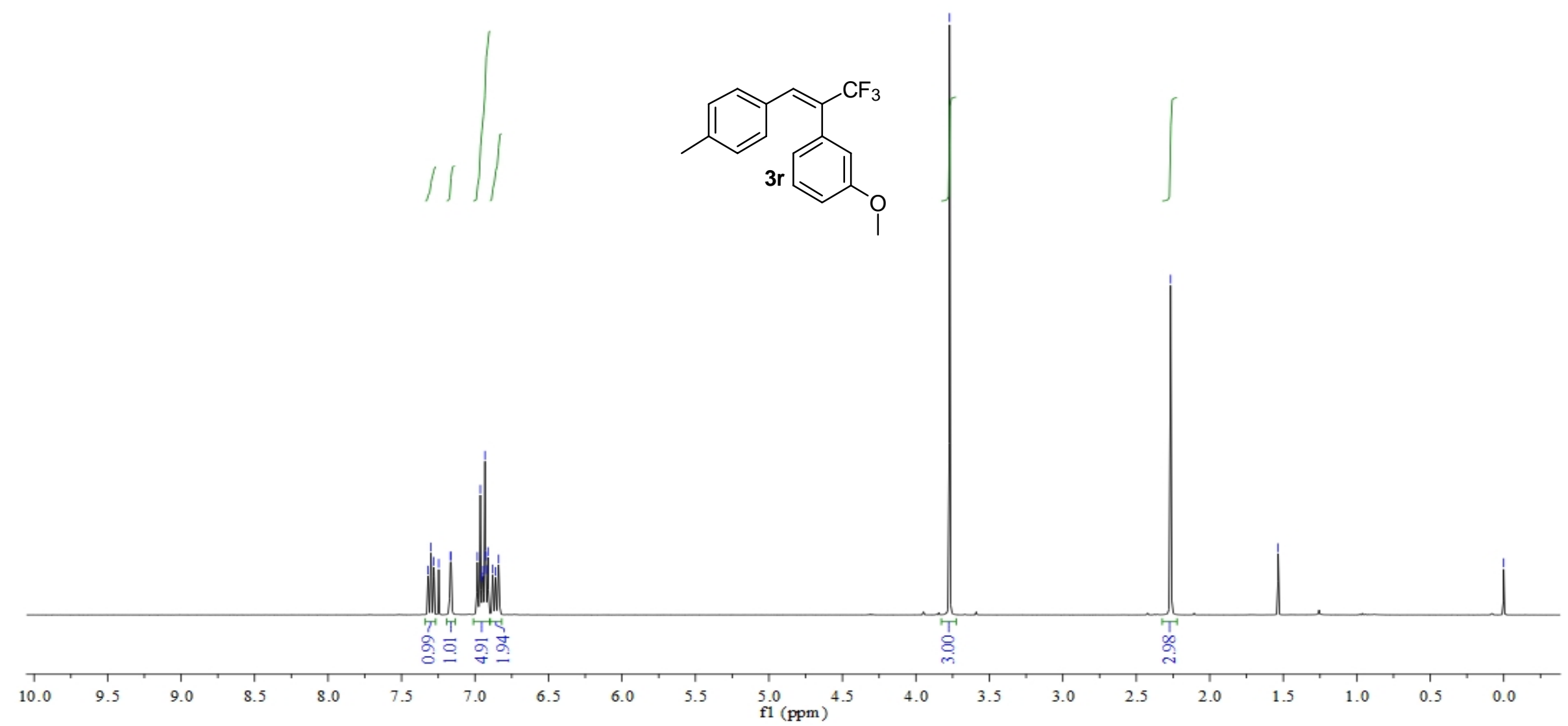




\section{${ }^{13}$ C-NMR}

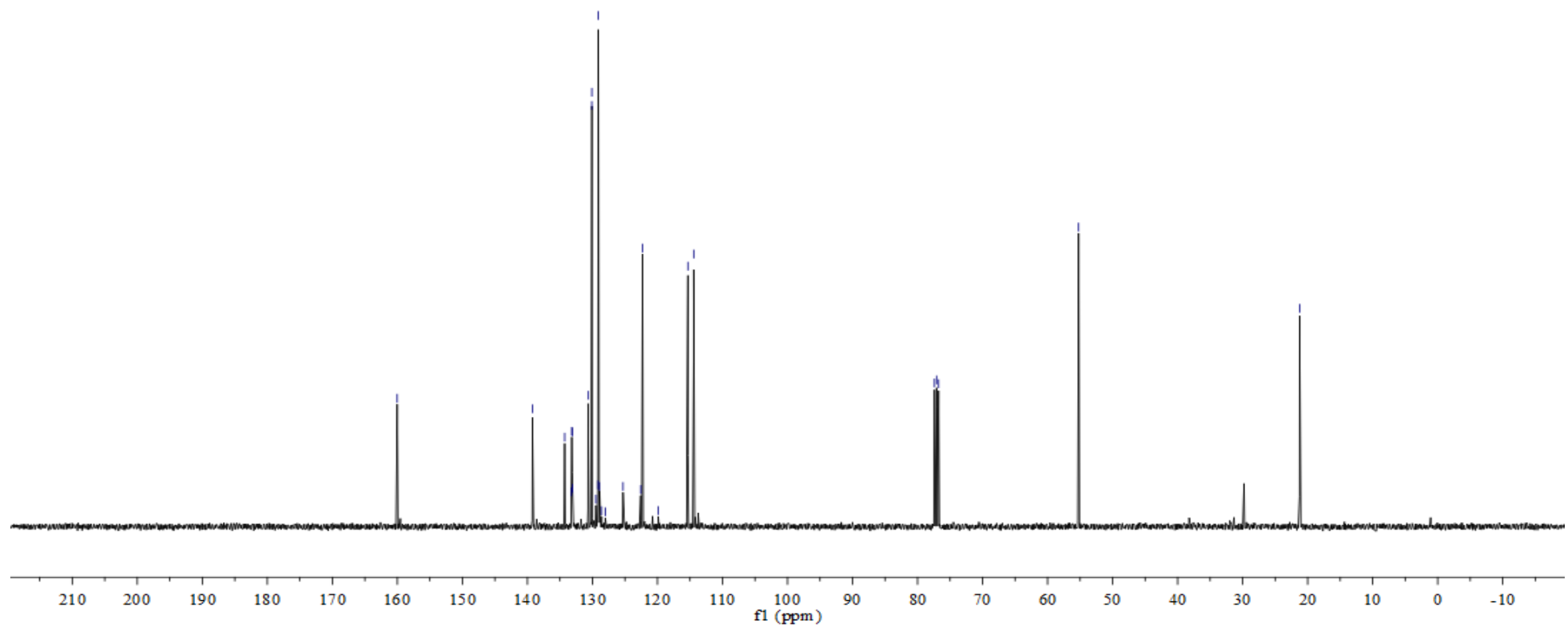


${ }^{19}$ F-NMR

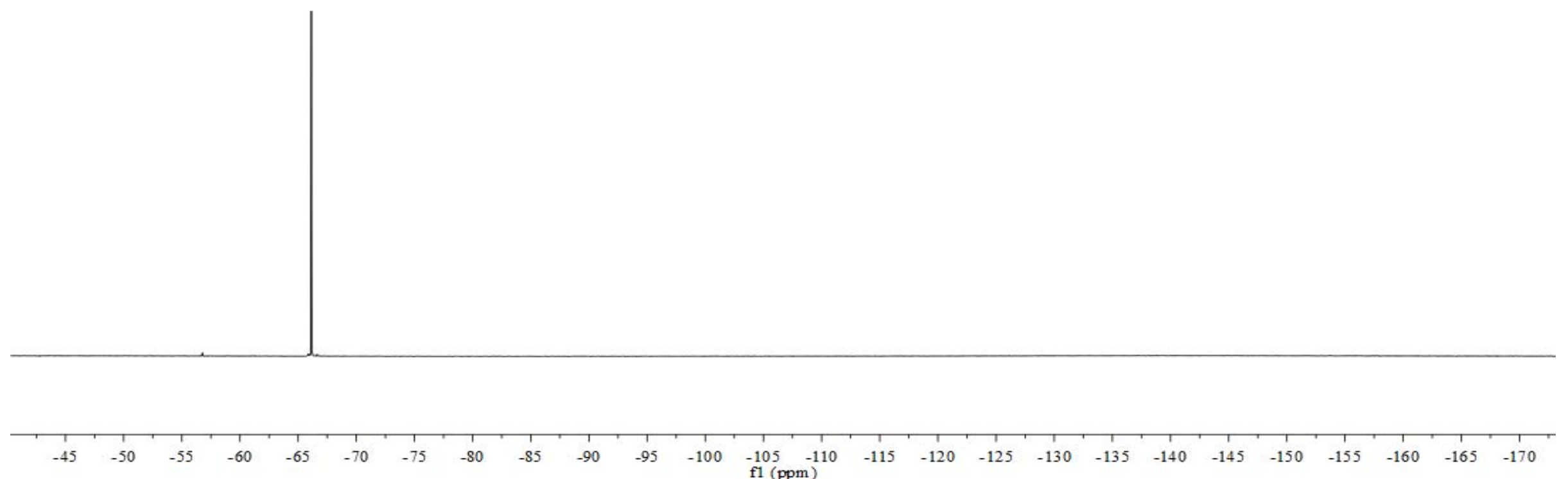




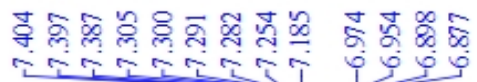

总

${ }^{1}$ H-NMR
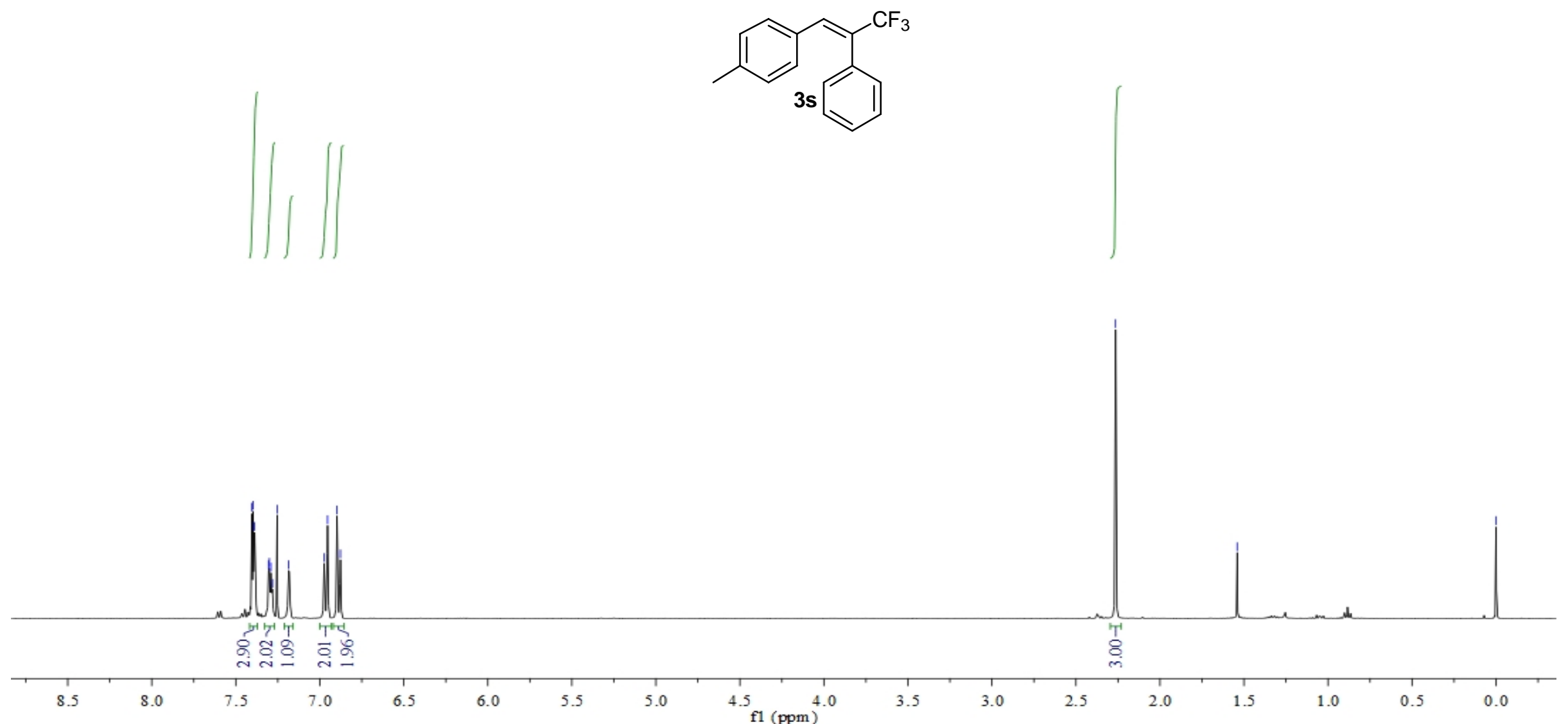
${ }^{13}$ C-NMR

응

1

용

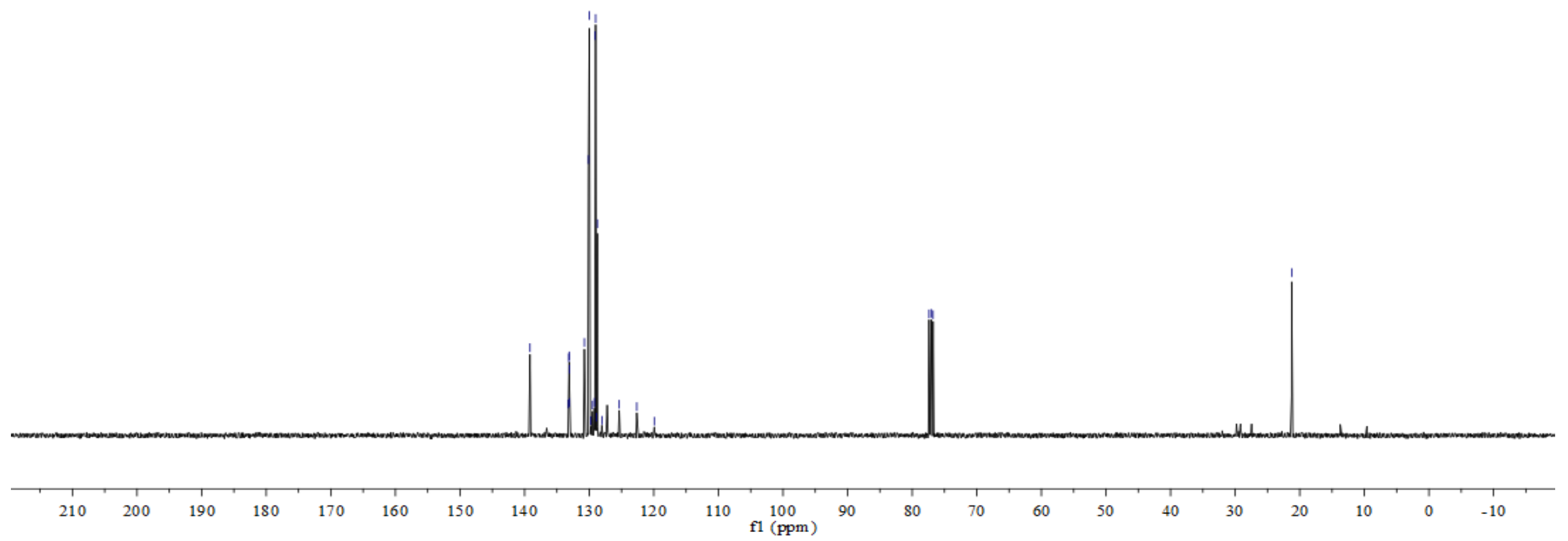


${ }^{19}$ F-NMR

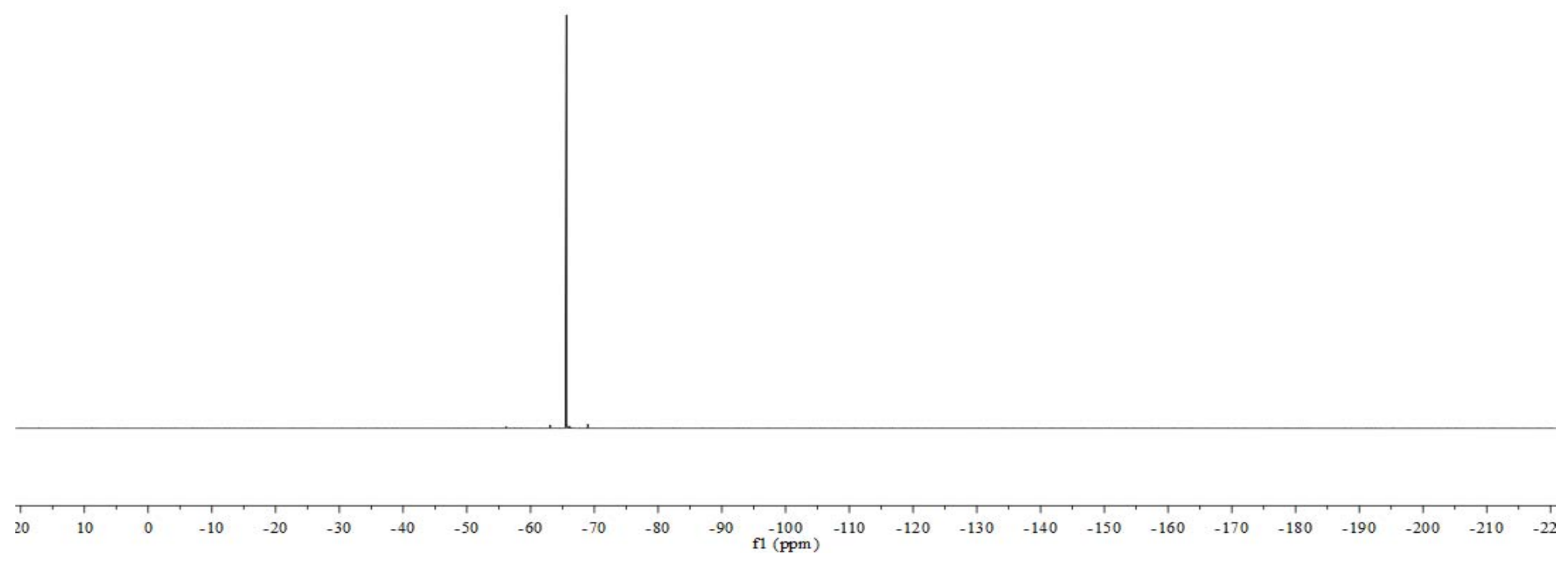




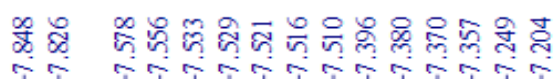

${ }^{1}$ H-NMR
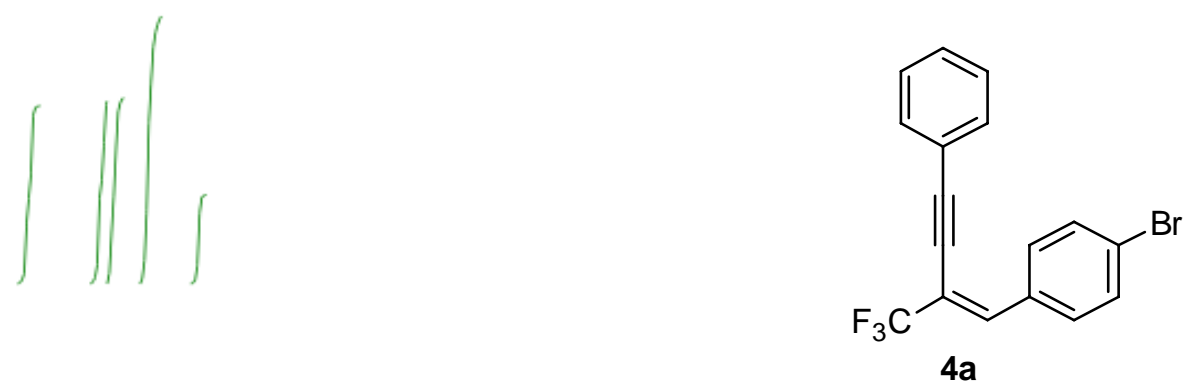

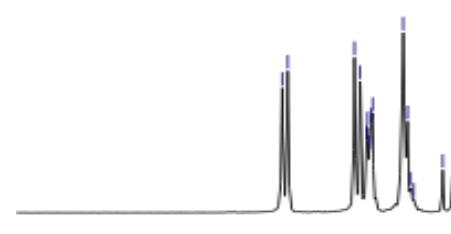

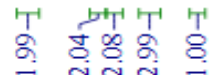

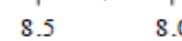

\begin{tabular}{lll}
\hline & 0 & \\
& 7.5 & 7.0
\end{tabular}

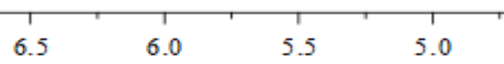

4.5 


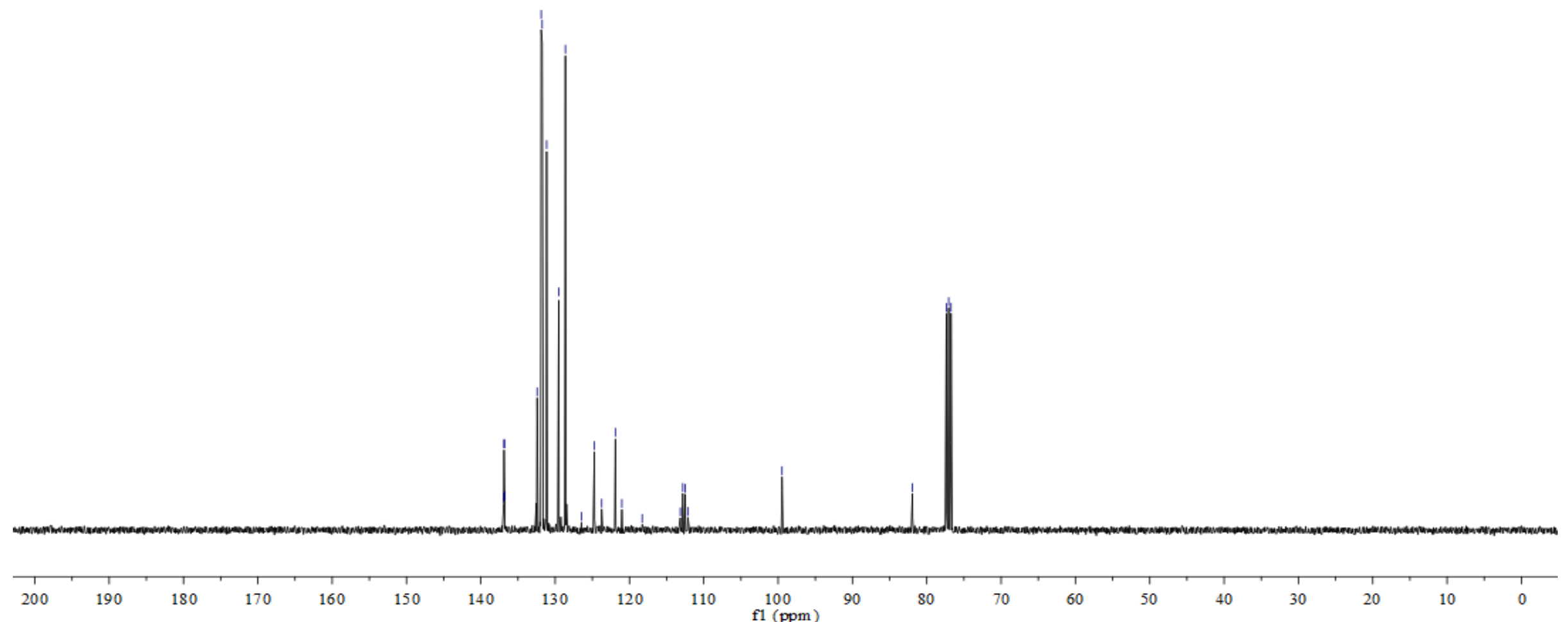


${ }^{19}$ F-NMR

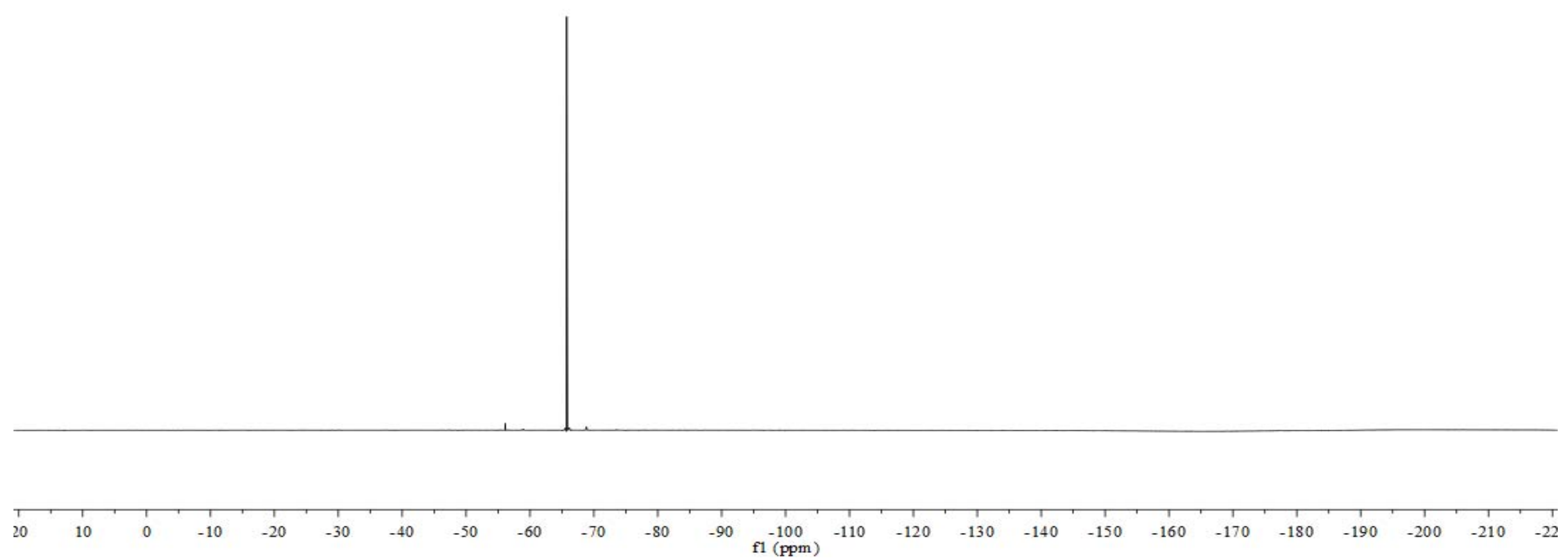




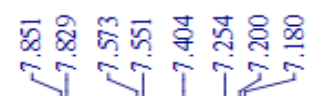

$\underset{\substack{\infty \\ \uparrow}}{\uparrow}$

${ }^{1}$ H-NMR
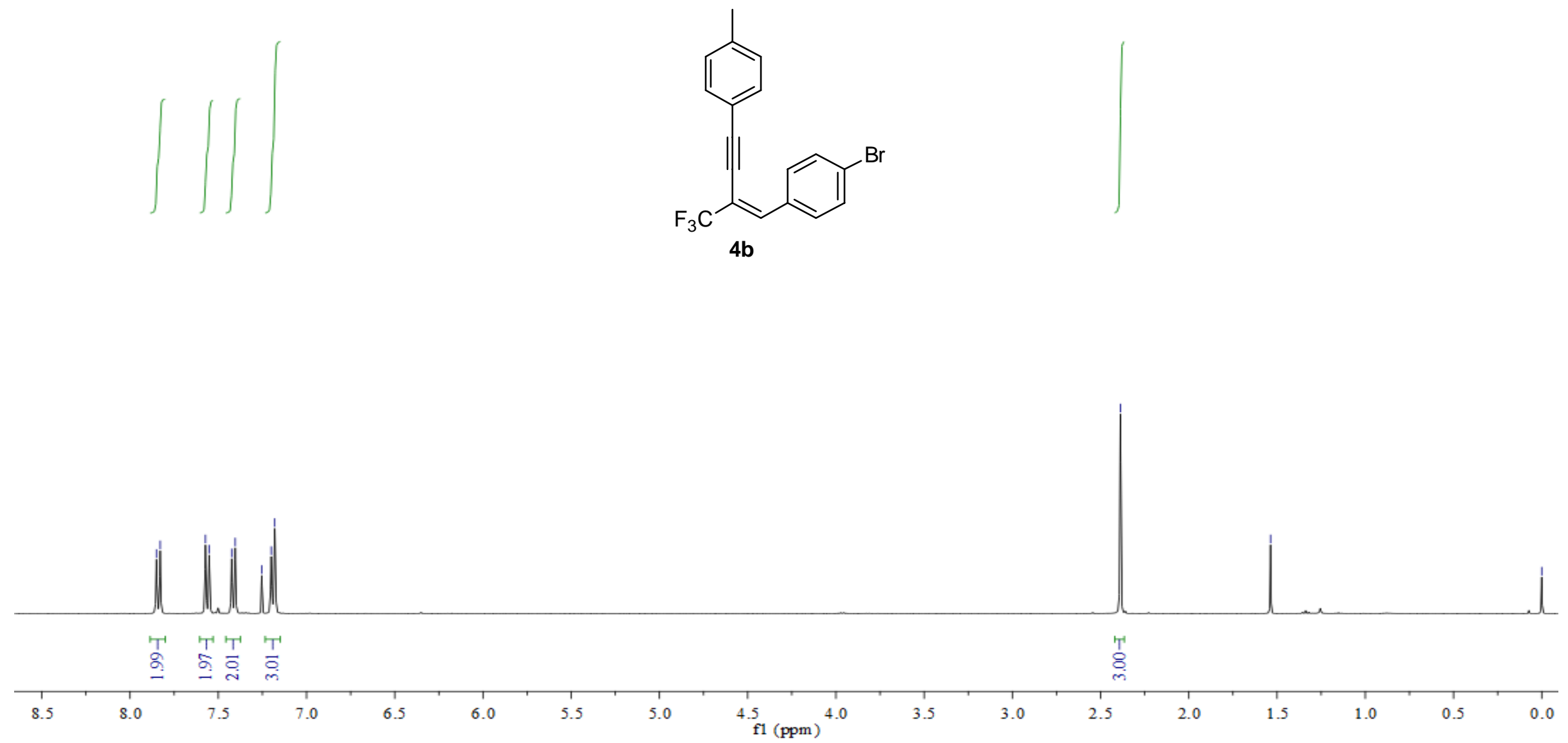


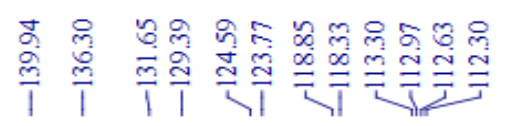

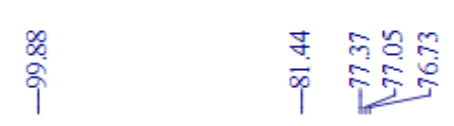

$\stackrel{\leftarrow}{\top}$

${ }^{13}$ C-NMR

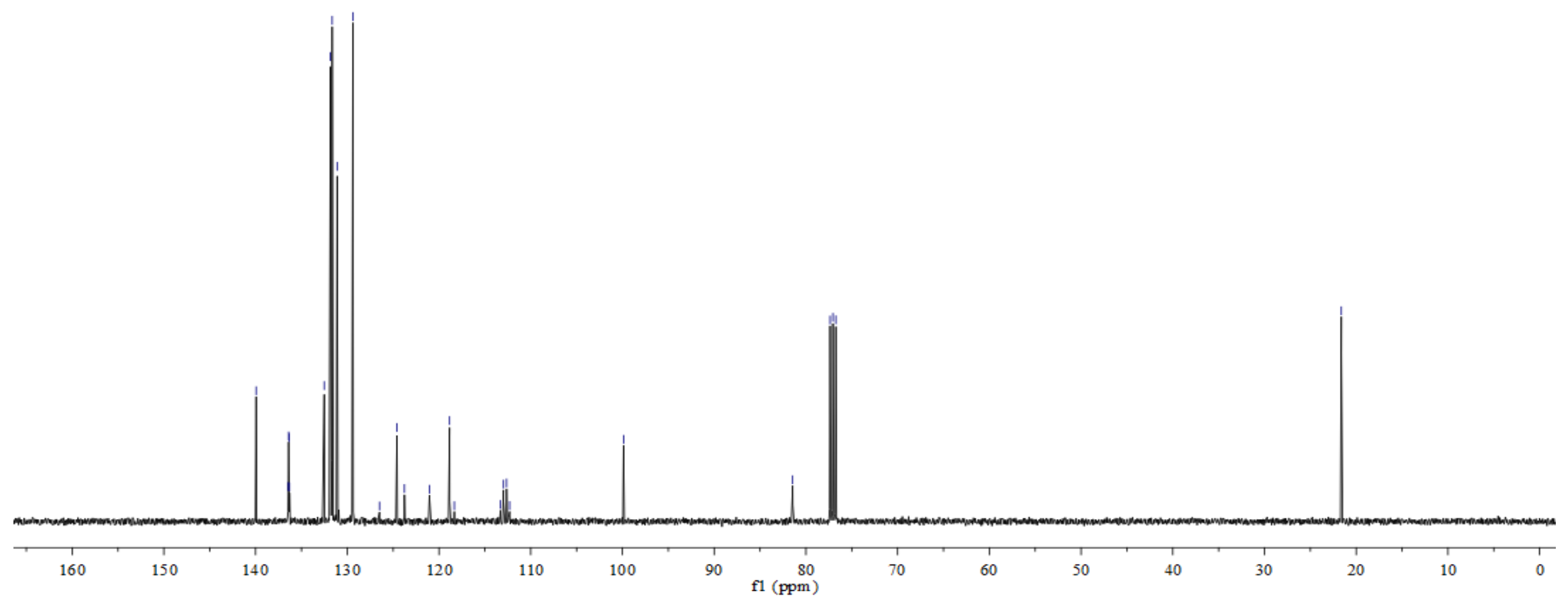

S118 
${ }^{19}$ F-NMR

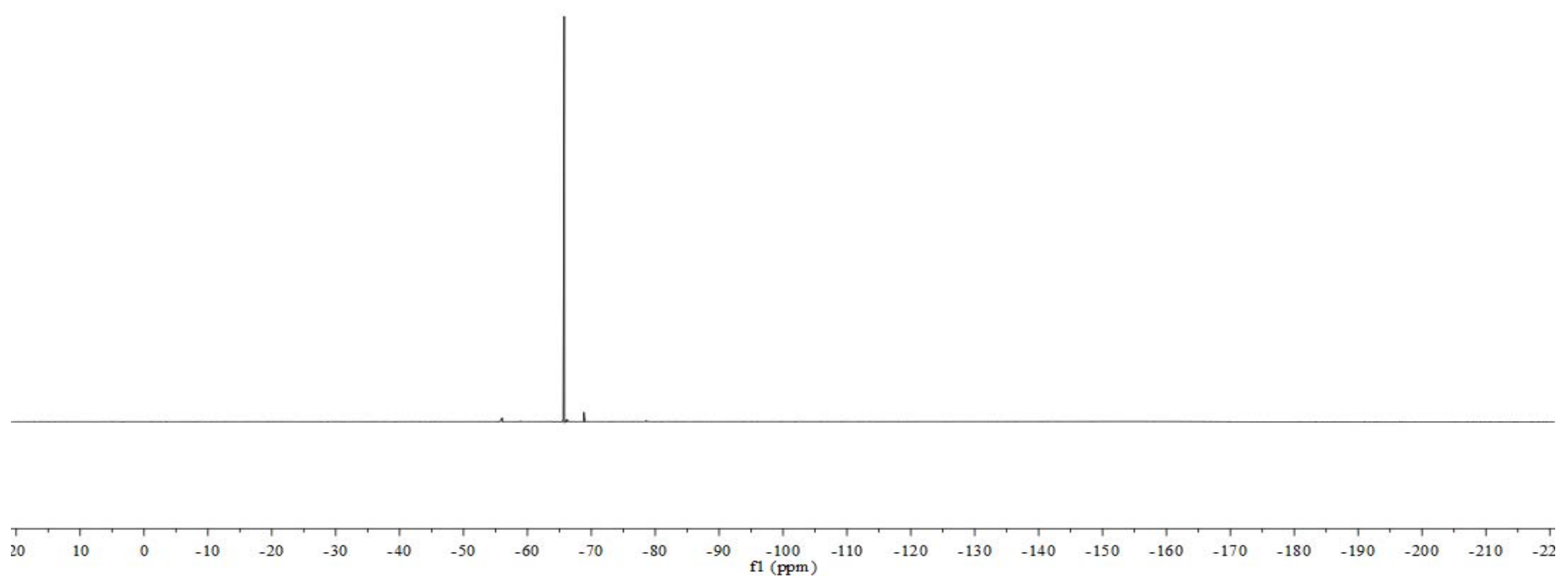



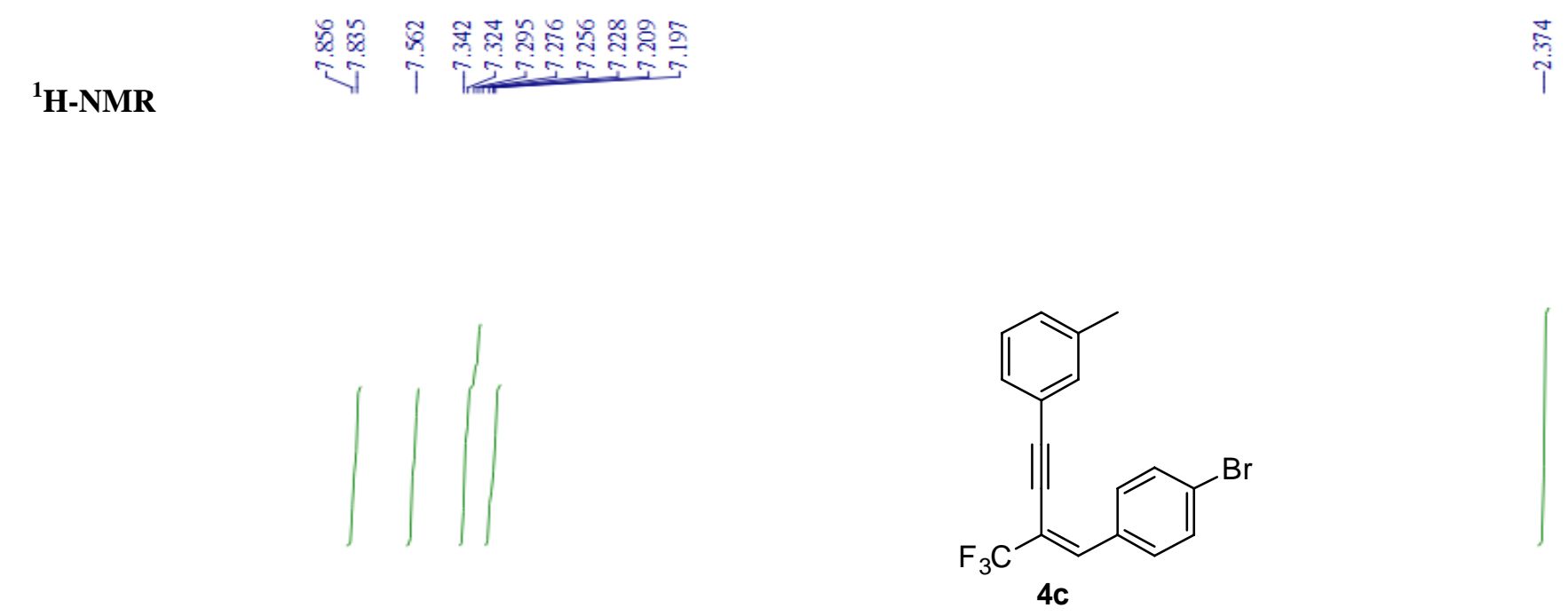

4c

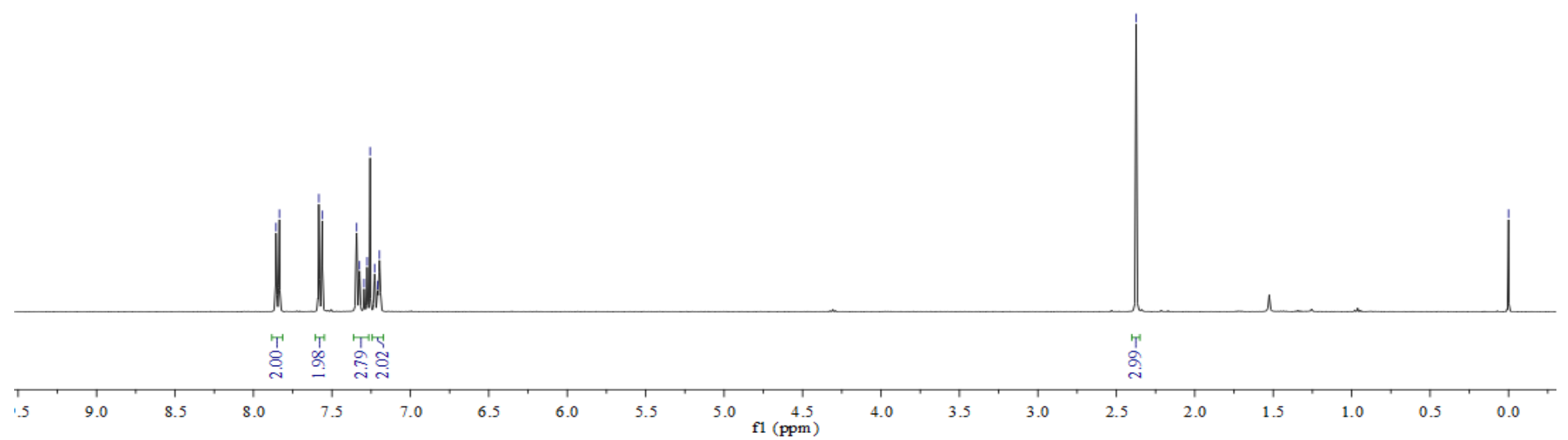



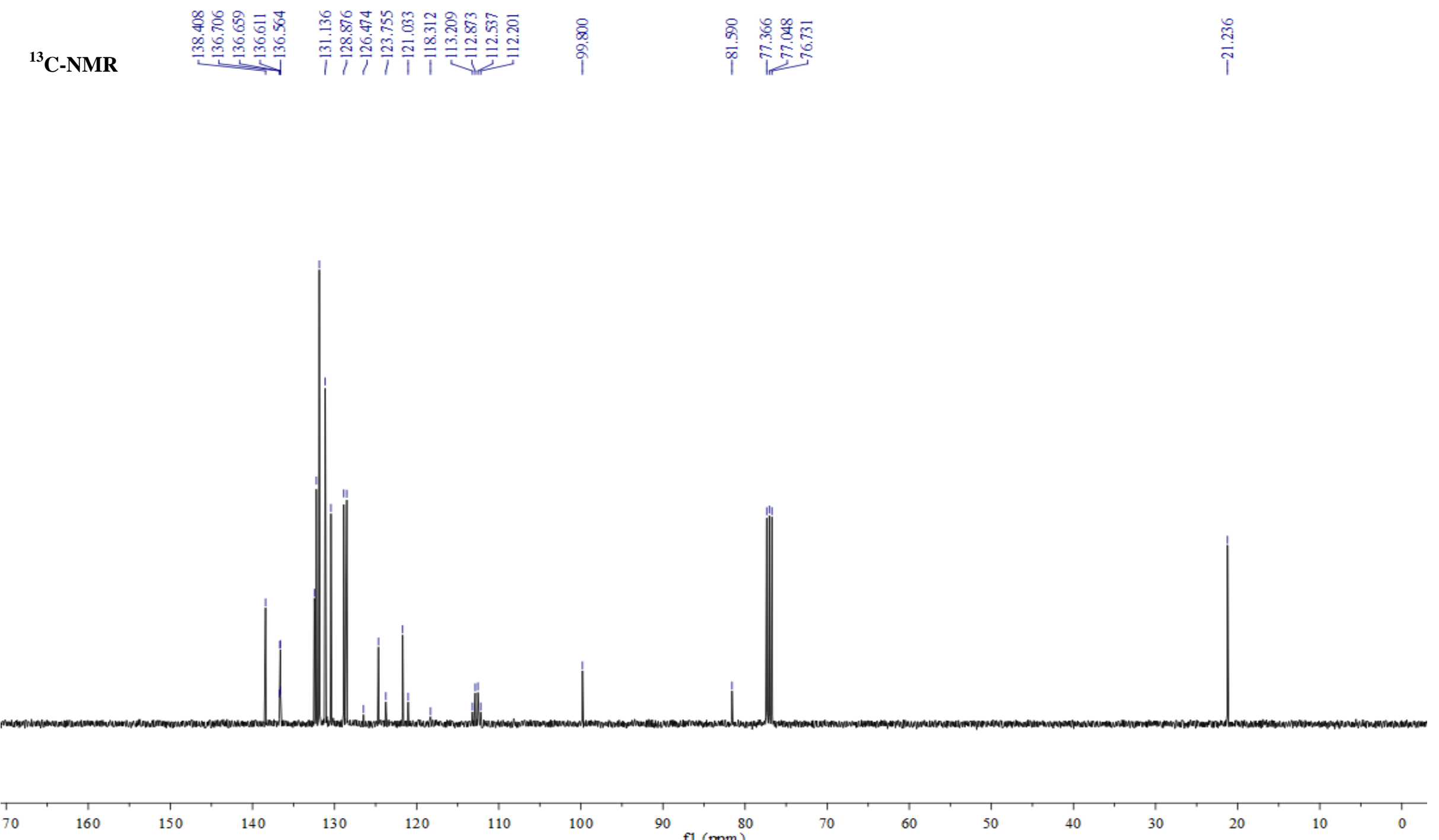
${ }^{19}$ F-NMR

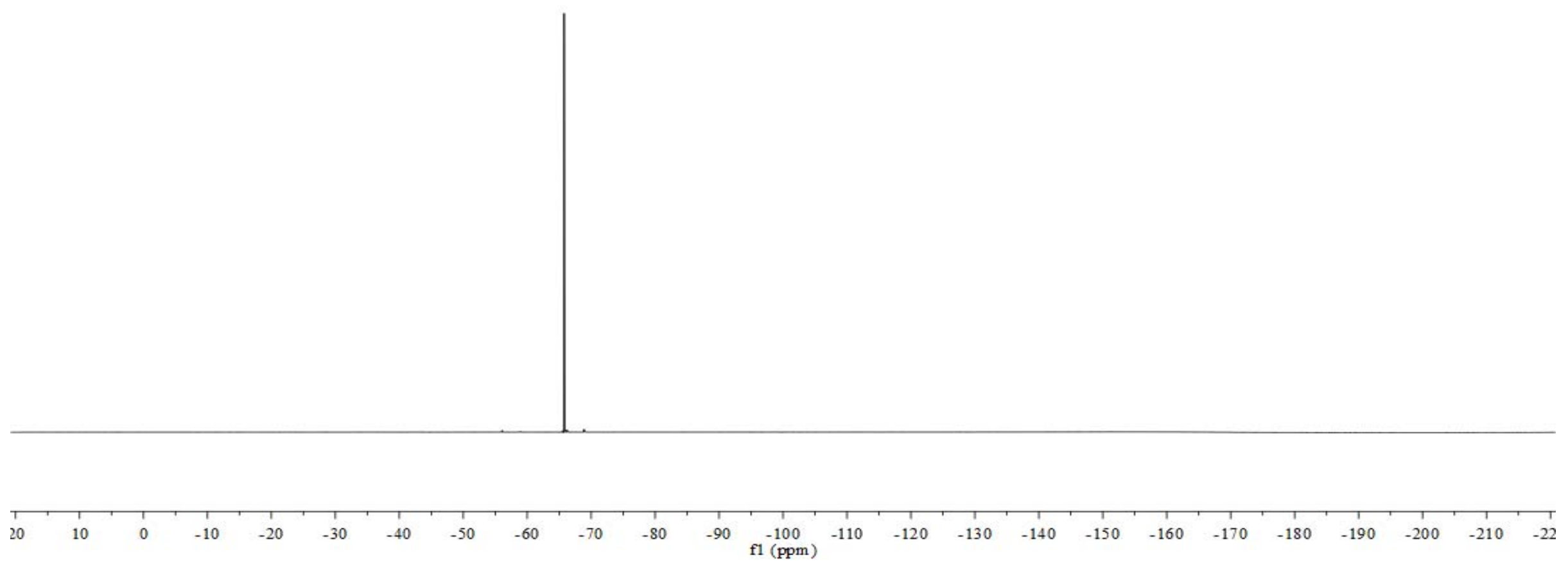




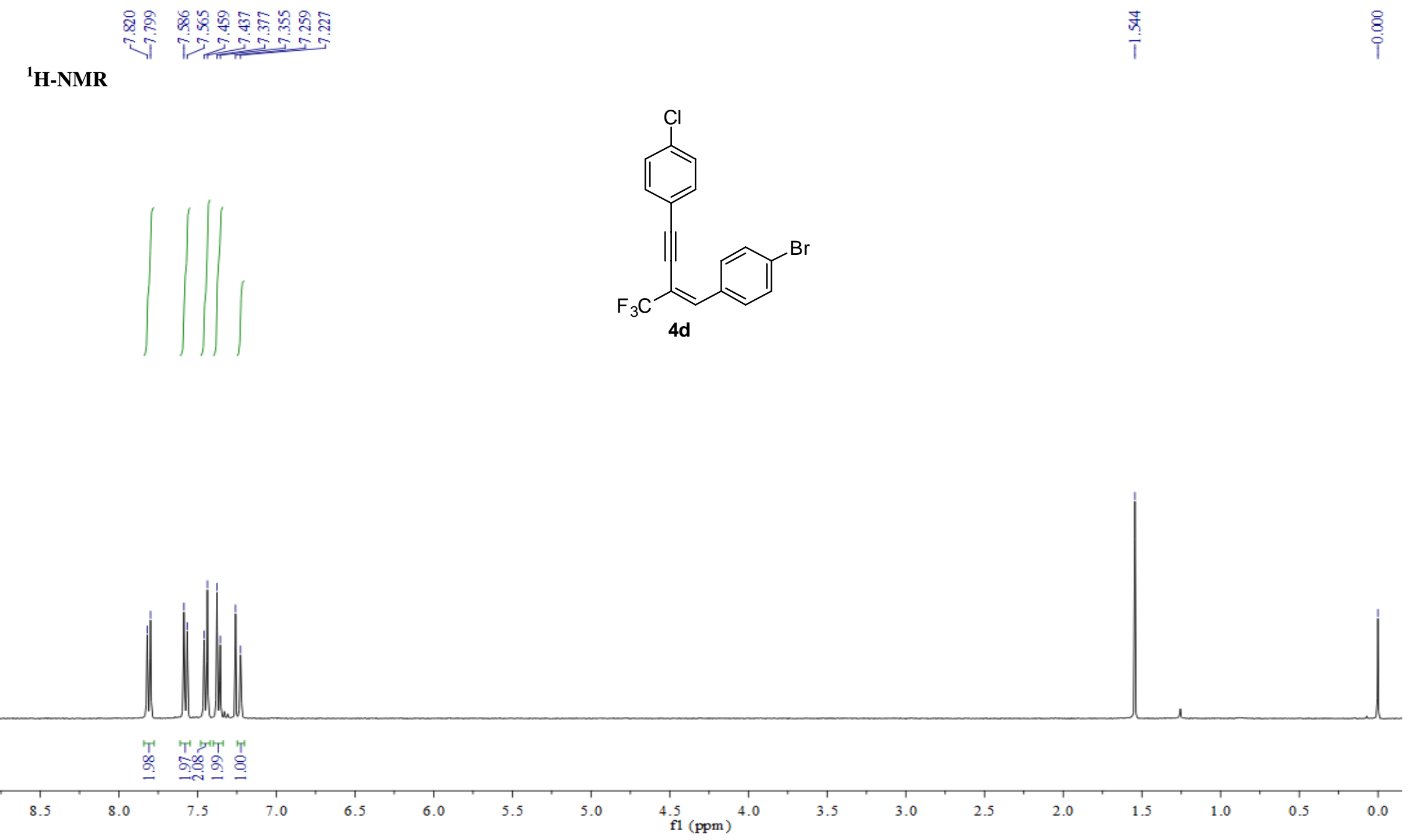


${ }^{13}$ C-NMR

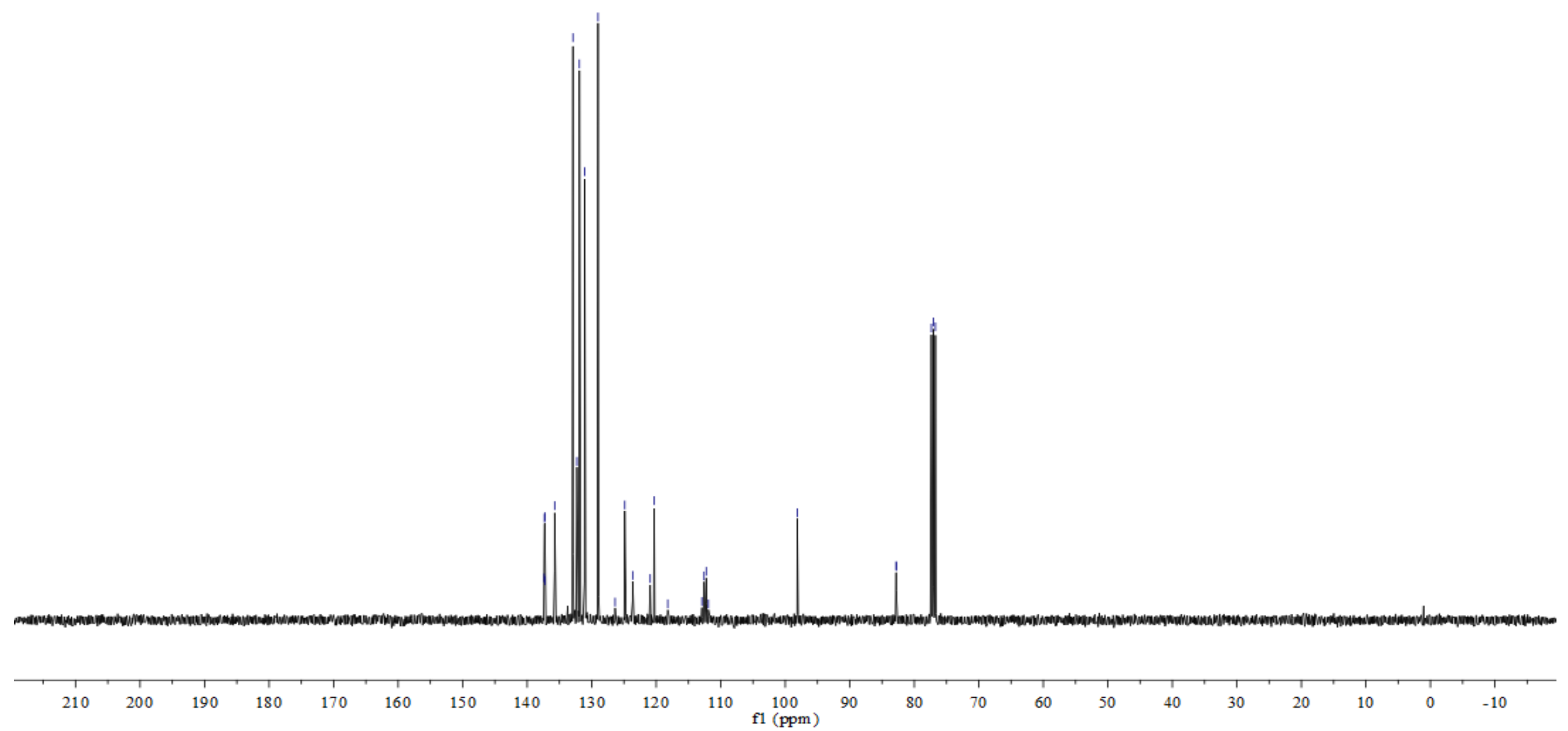


${ }^{19}$ F-NMR

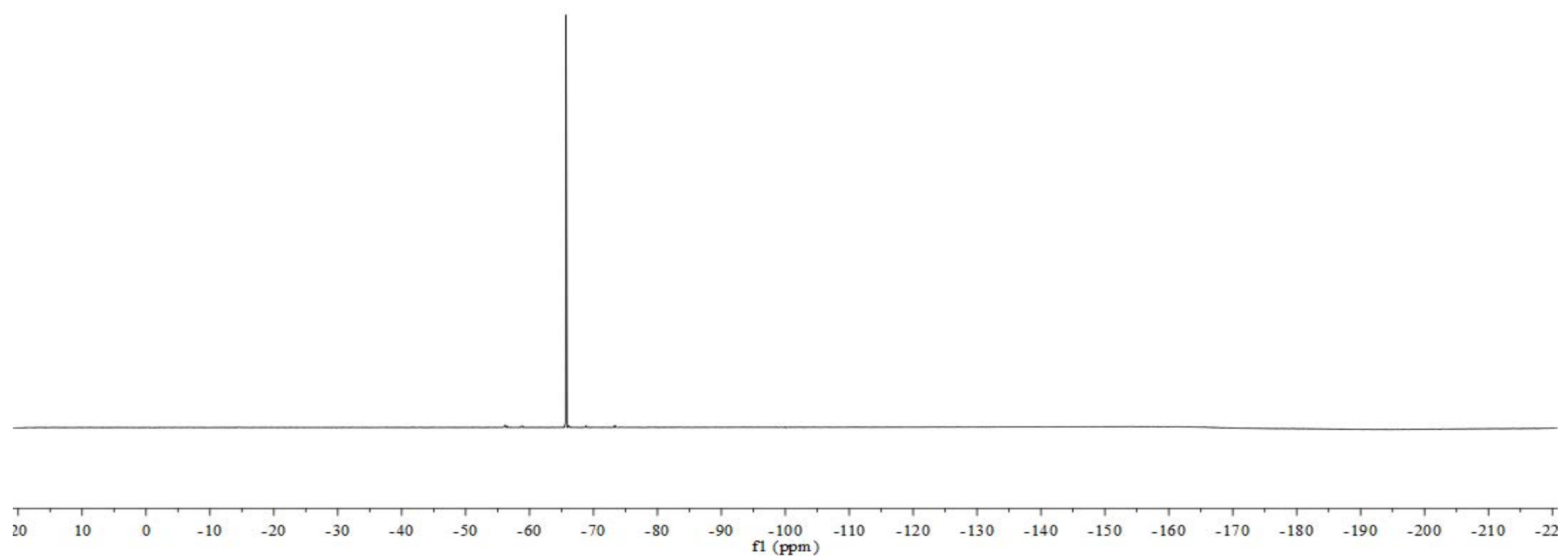




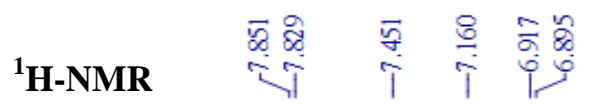
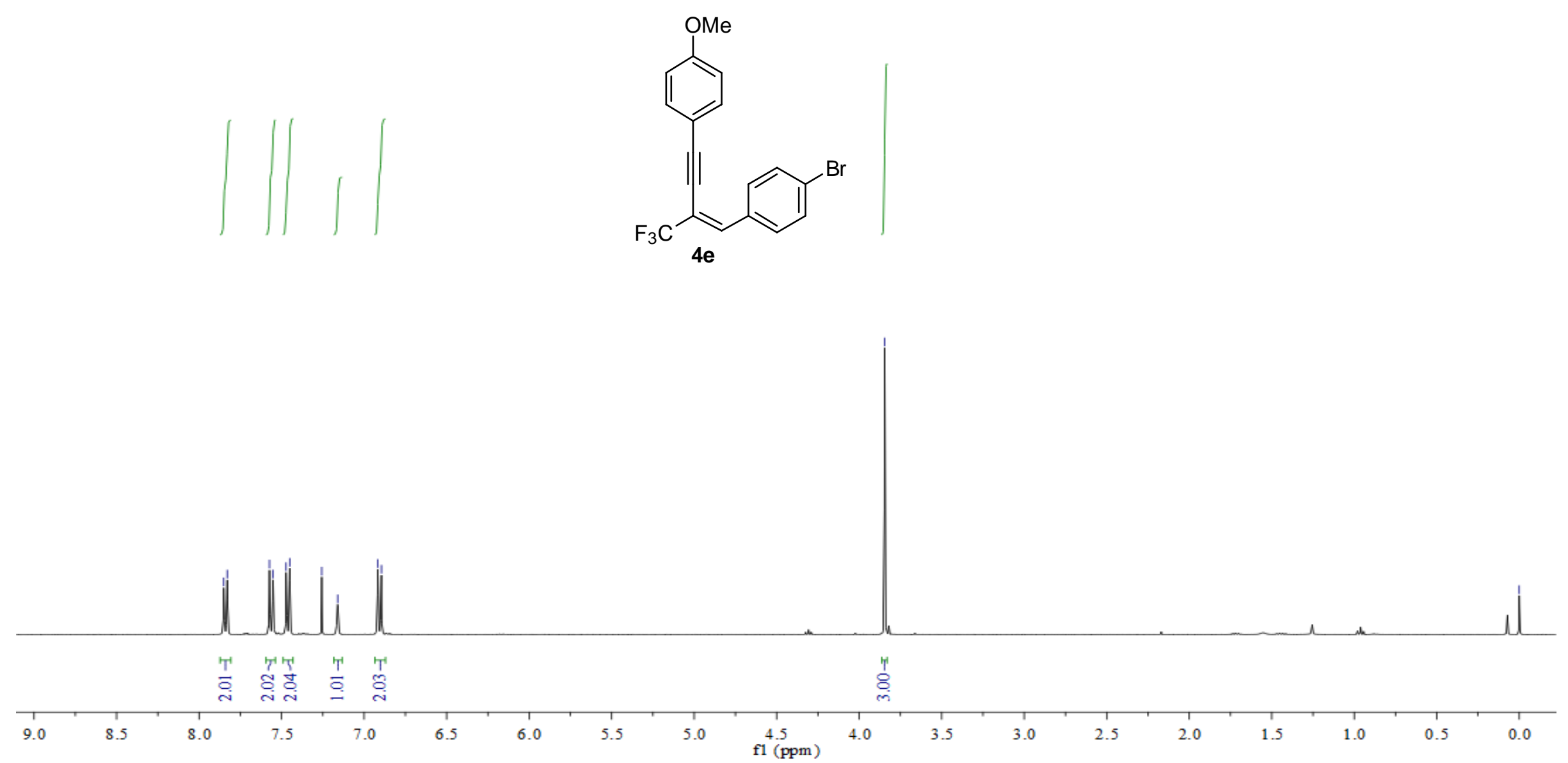
${ }^{13}$ C-NMR

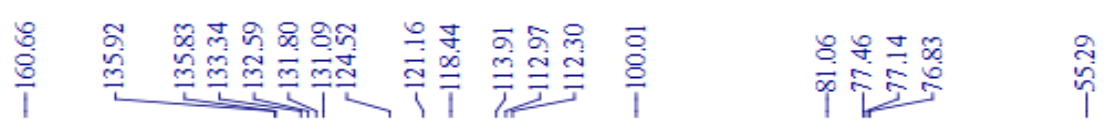

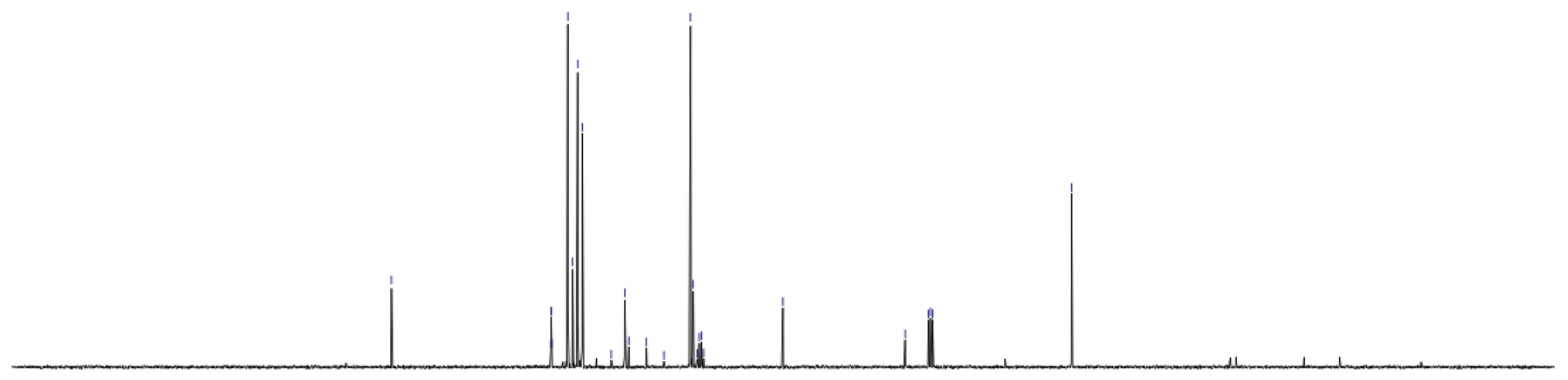

$210 \quad 200$ 
${ }^{19}$ F-NMR

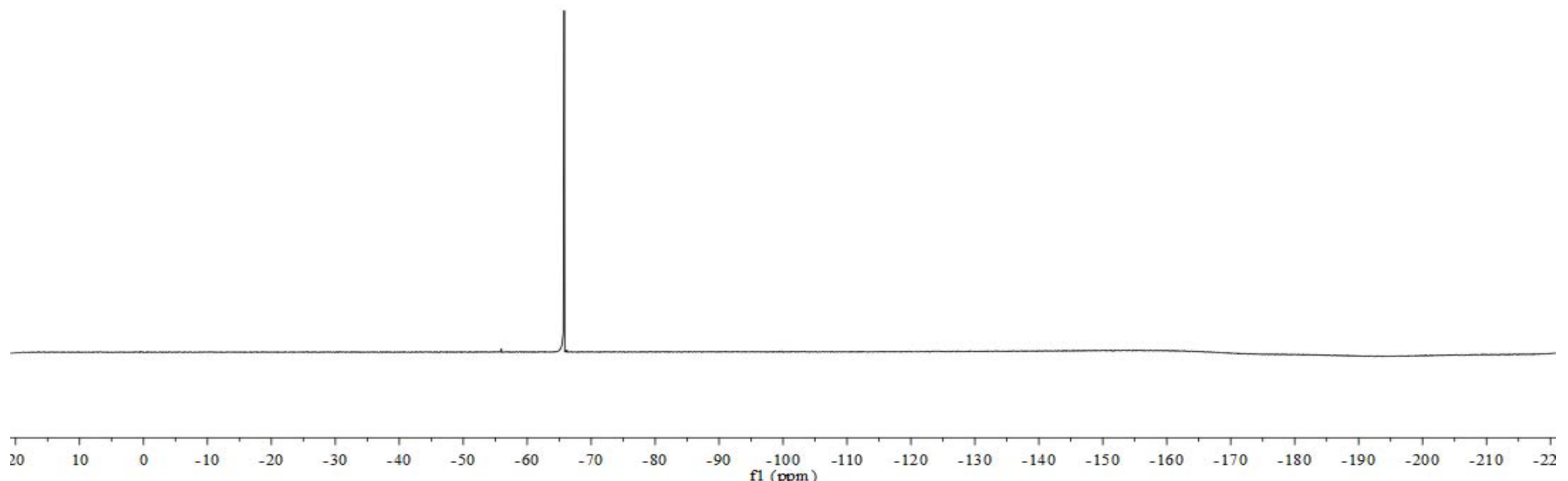


${ }^{1}$ H-NMR

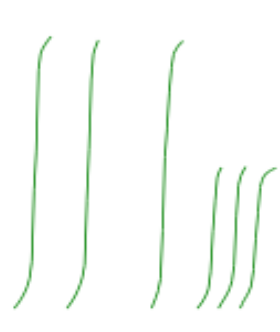

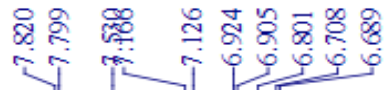

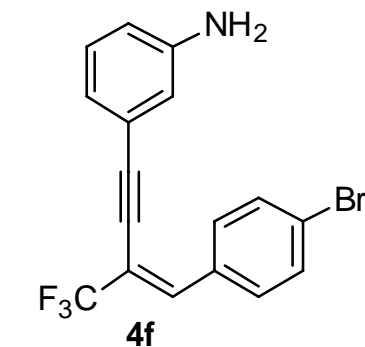

$4 f$

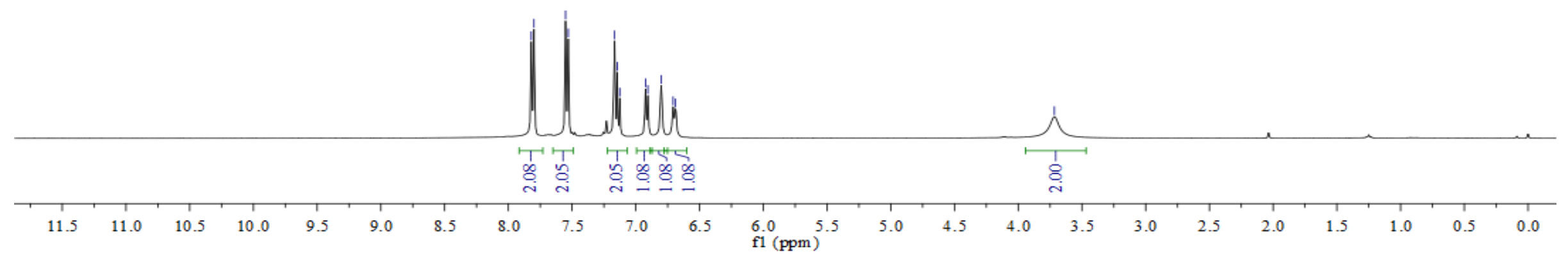


${ }^{13}$ C-NMR
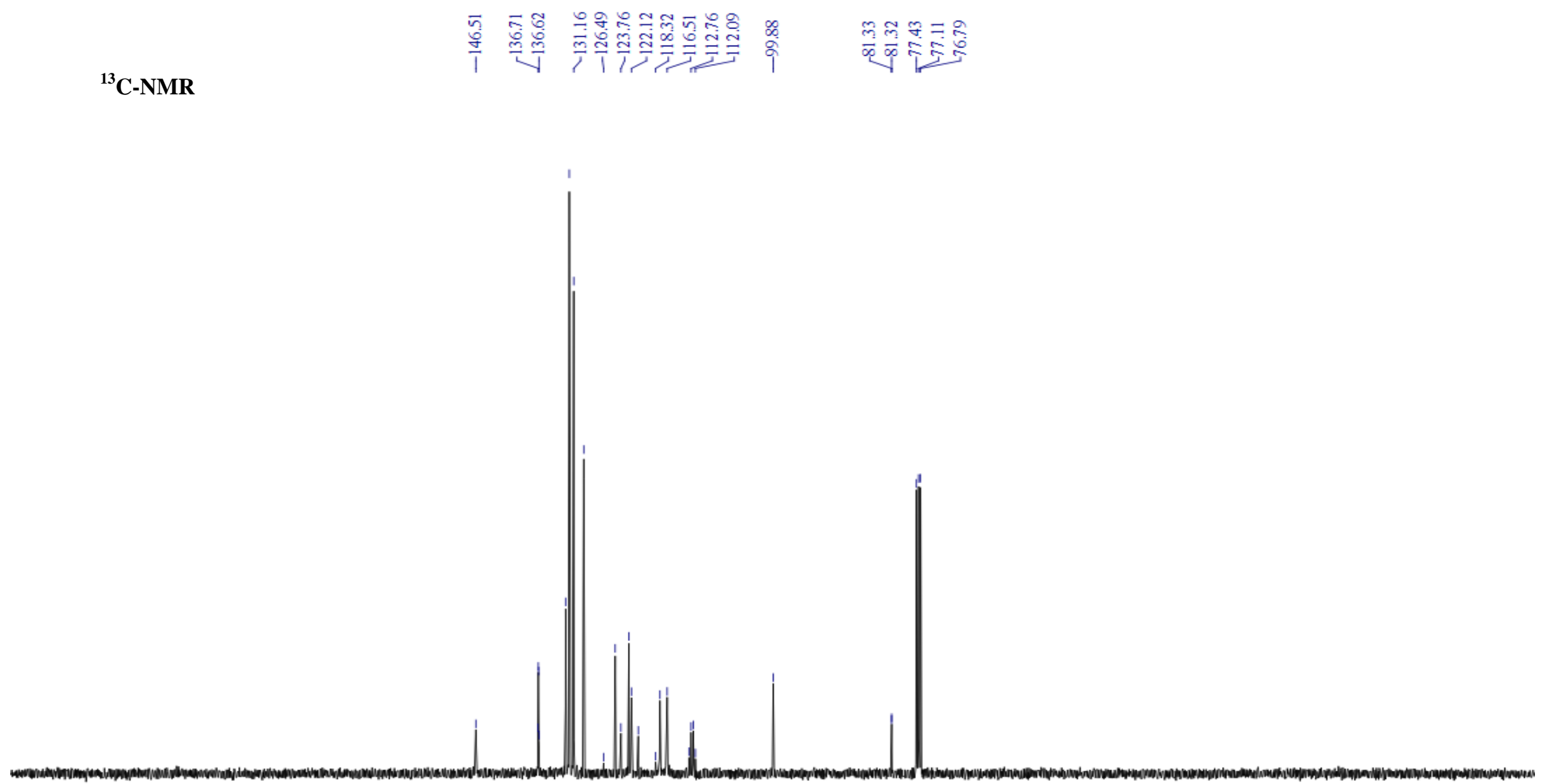

$\begin{array}{lllllllll}210 & 200 & 190 & 180 & 170 & 160 & 150 & 140 & 130\end{array}$

$110 \quad 100$

$\mathrm{f} 1(\mathrm{ppm})$

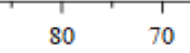

60

40 
${ }^{19}$ F-NMR

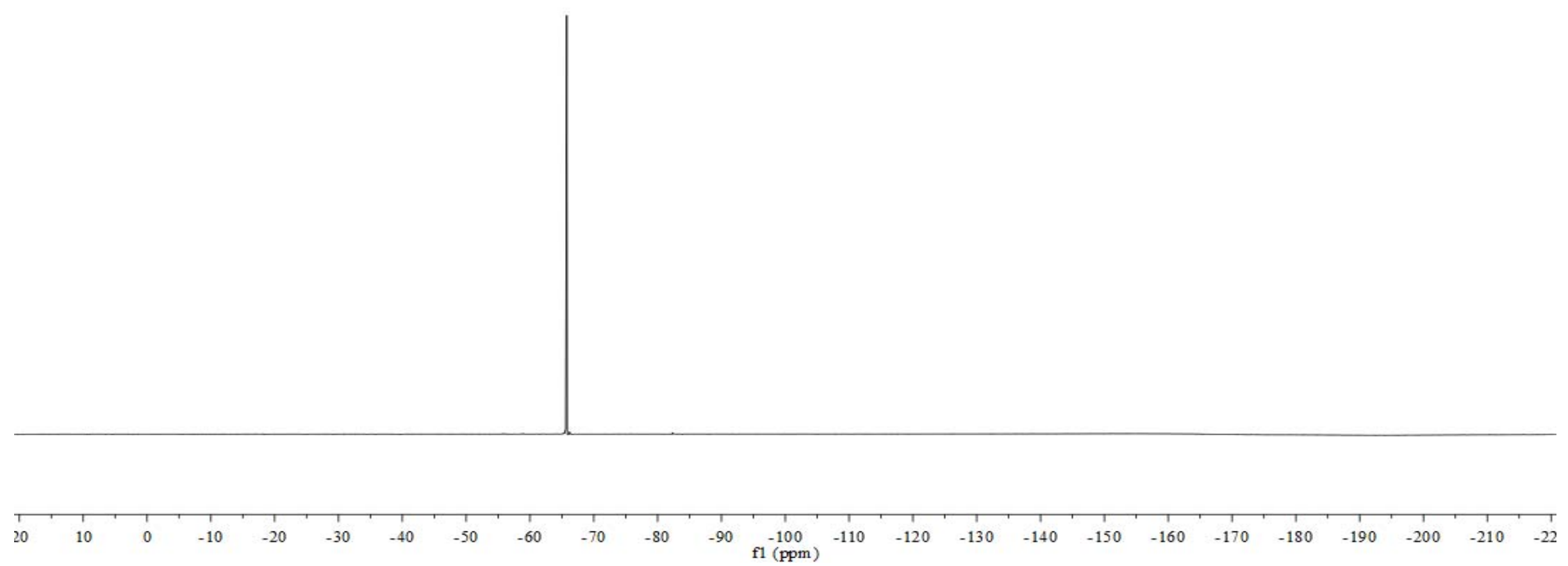



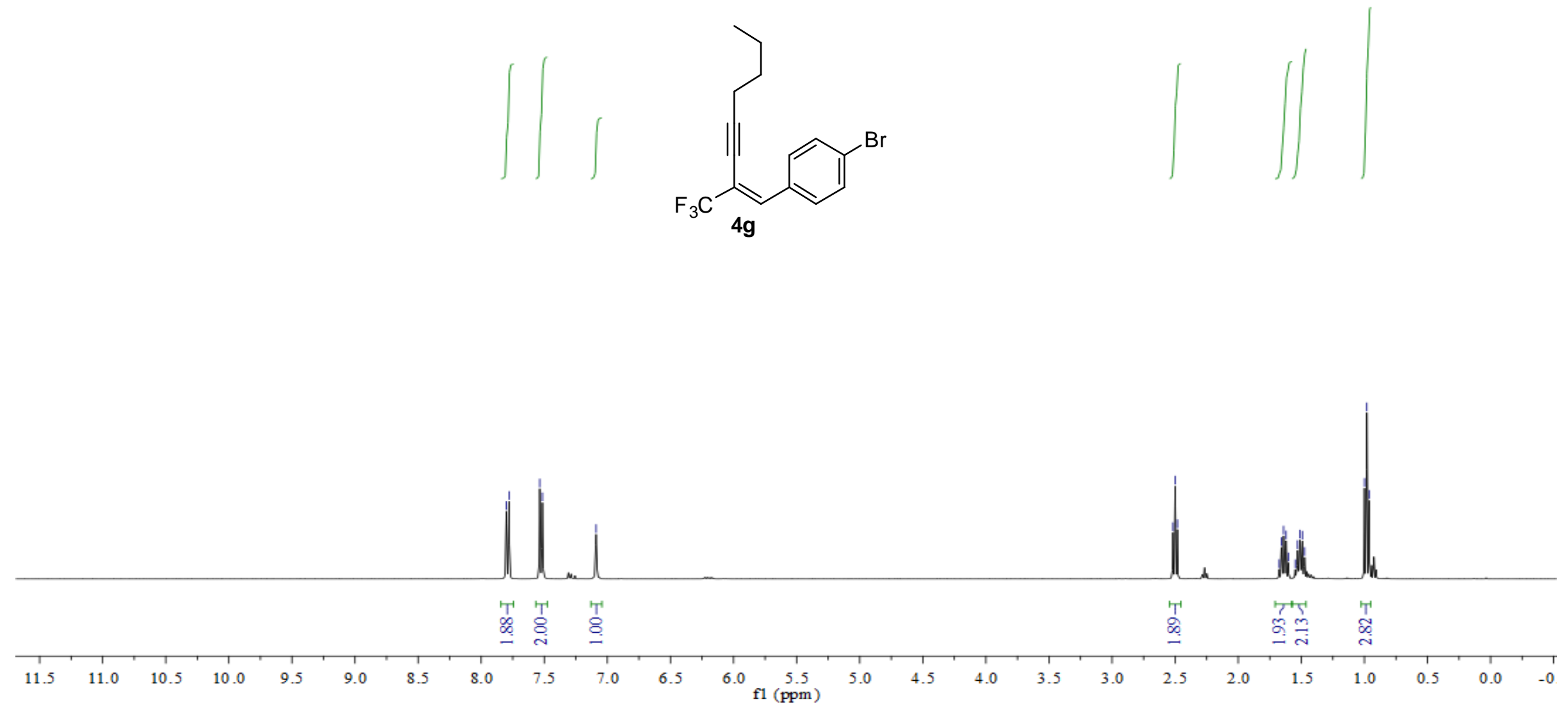


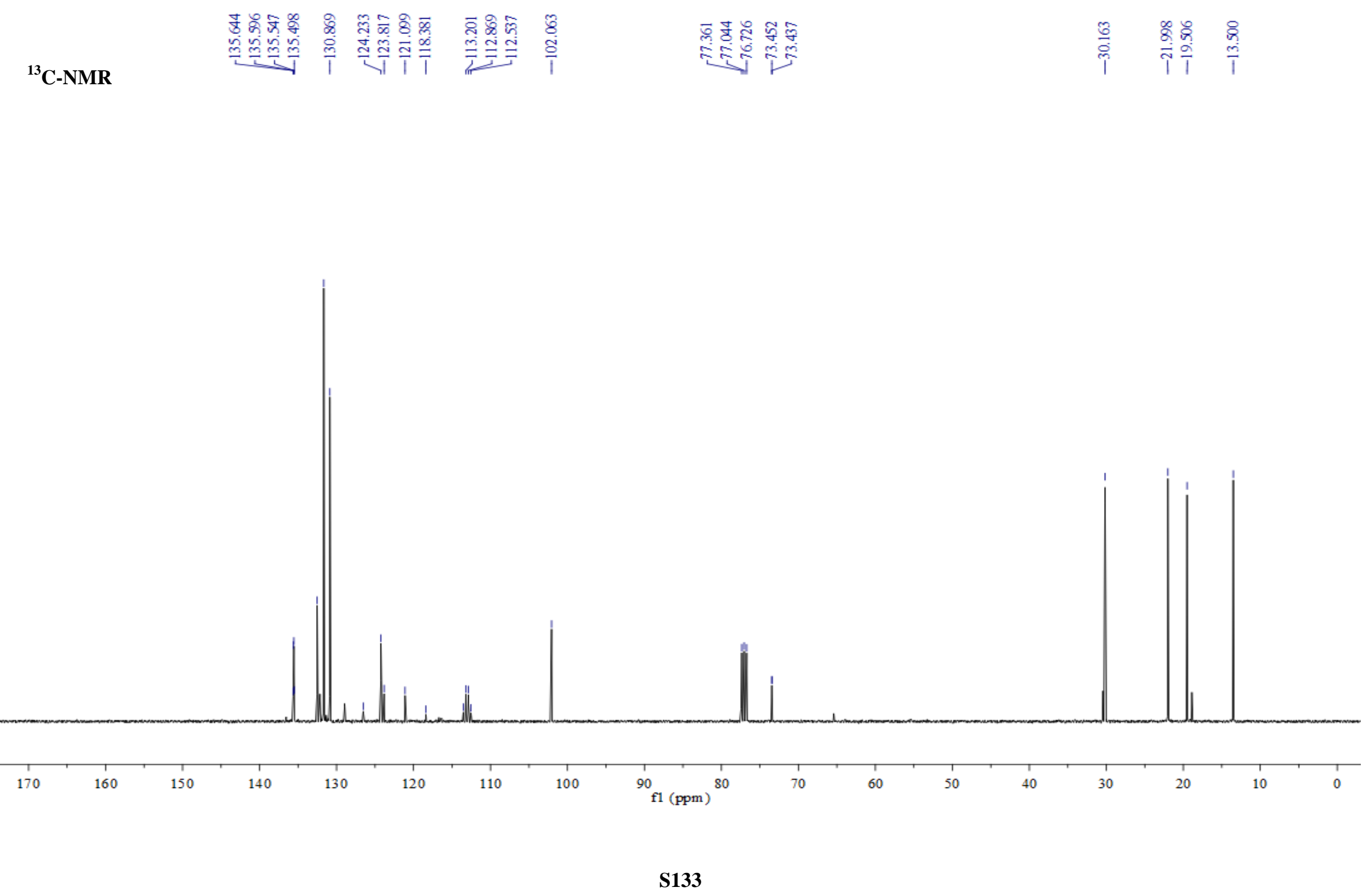


${ }^{19}$ F-NMR
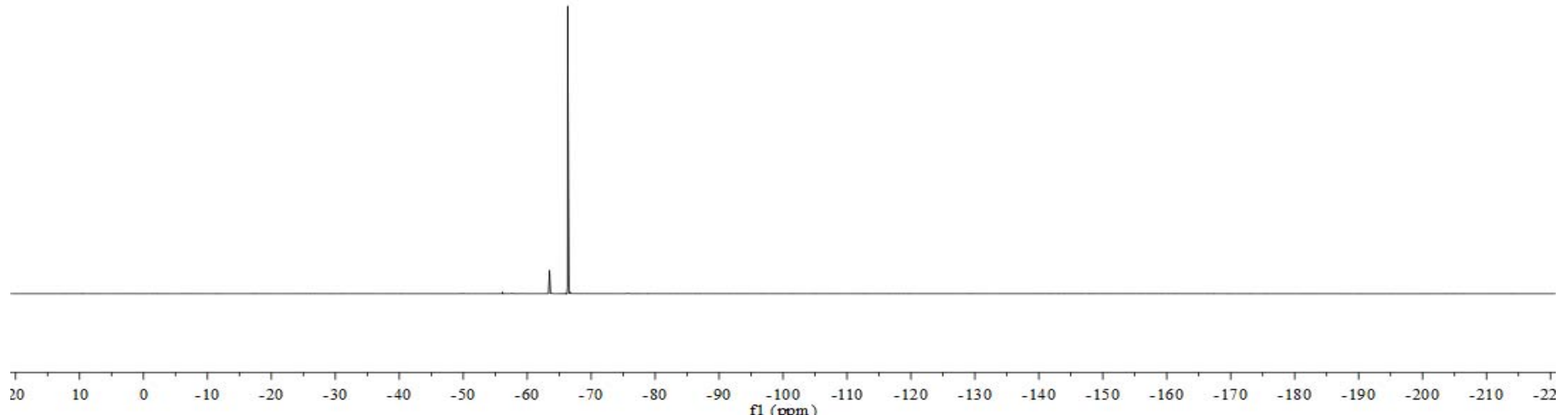


\section{${ }^{1}$ H-NMR

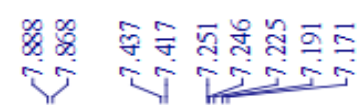
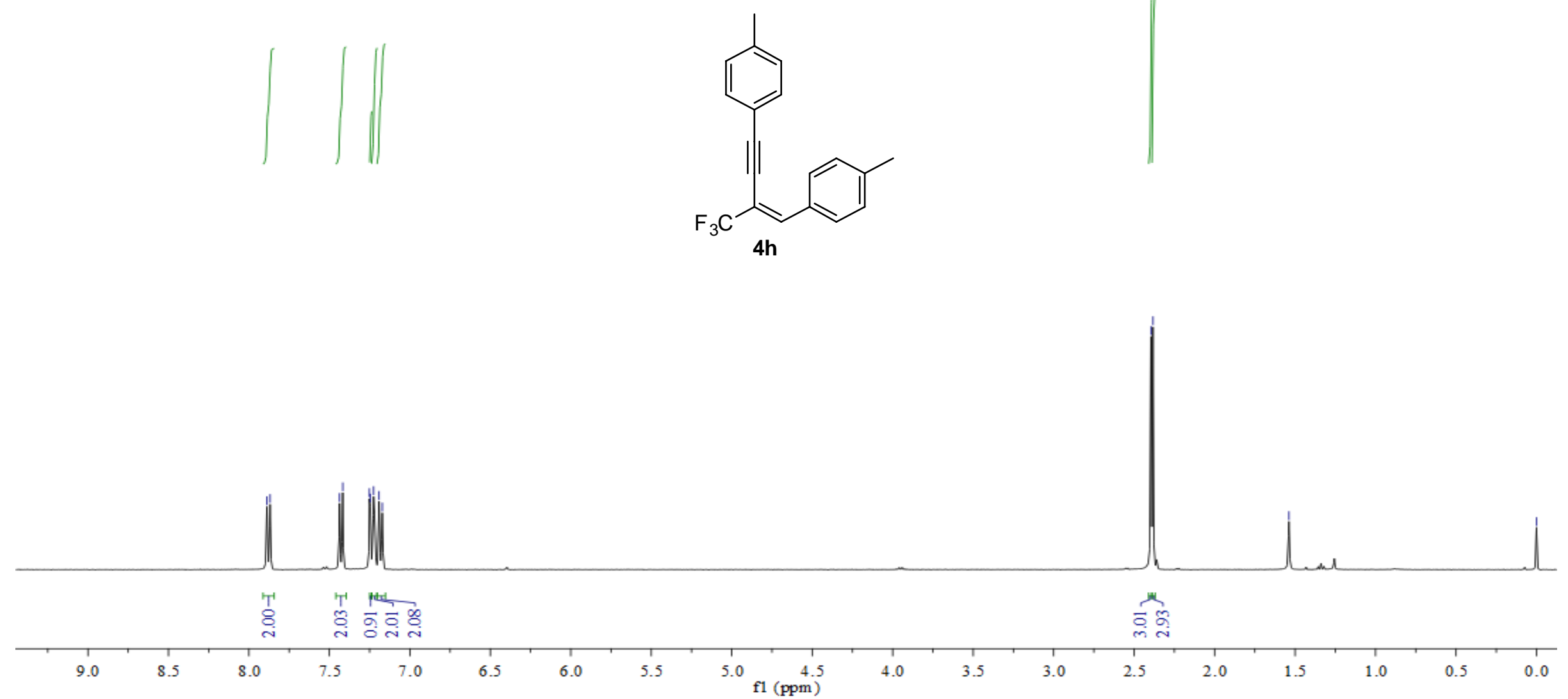
${ }^{13}$ C-NMR

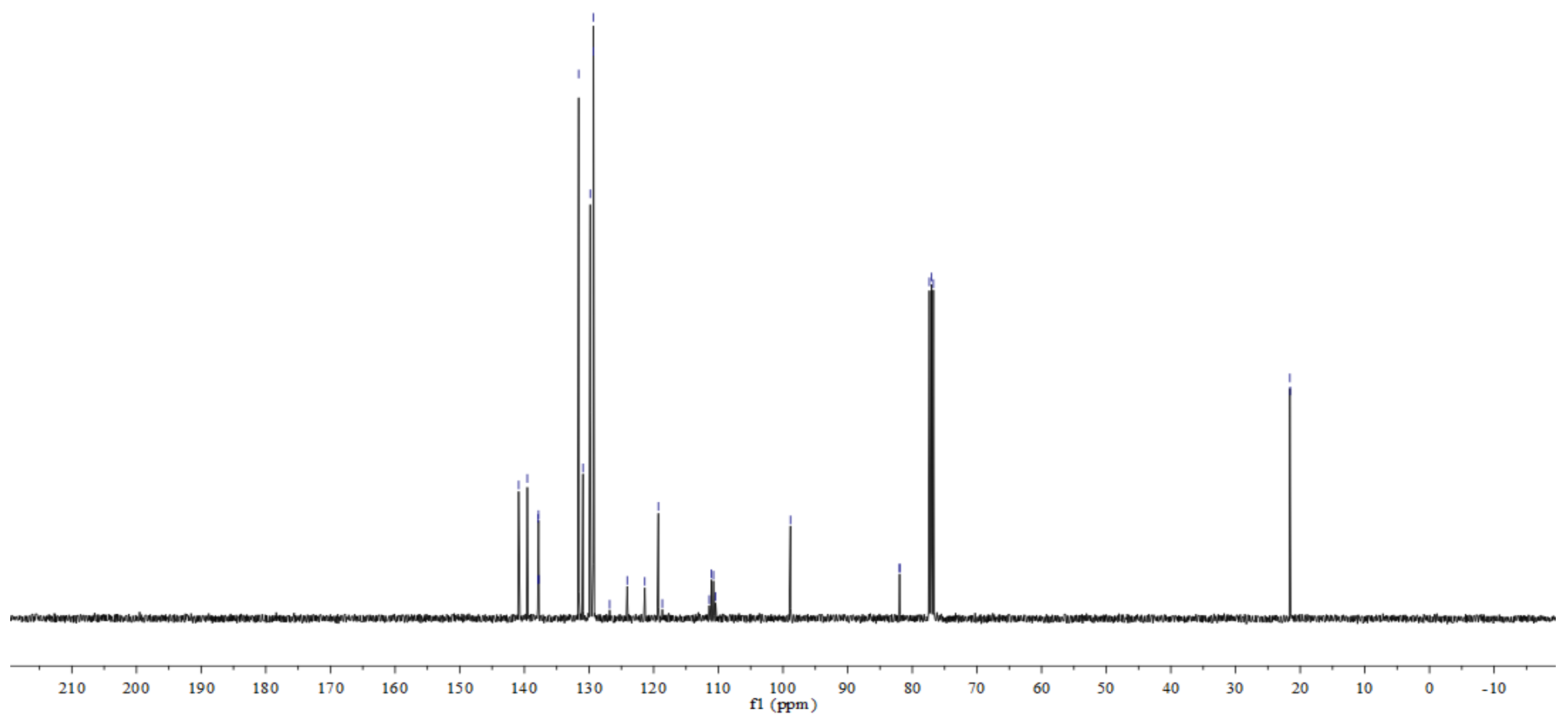


${ }^{19}$ F-NMR

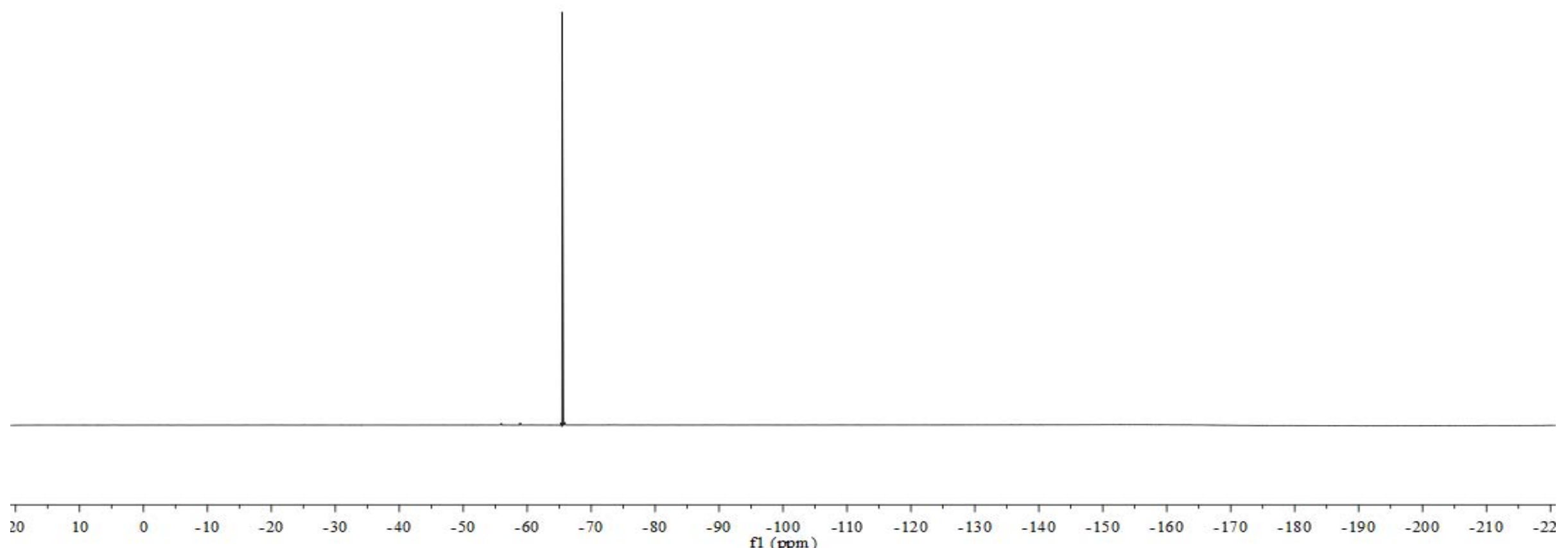



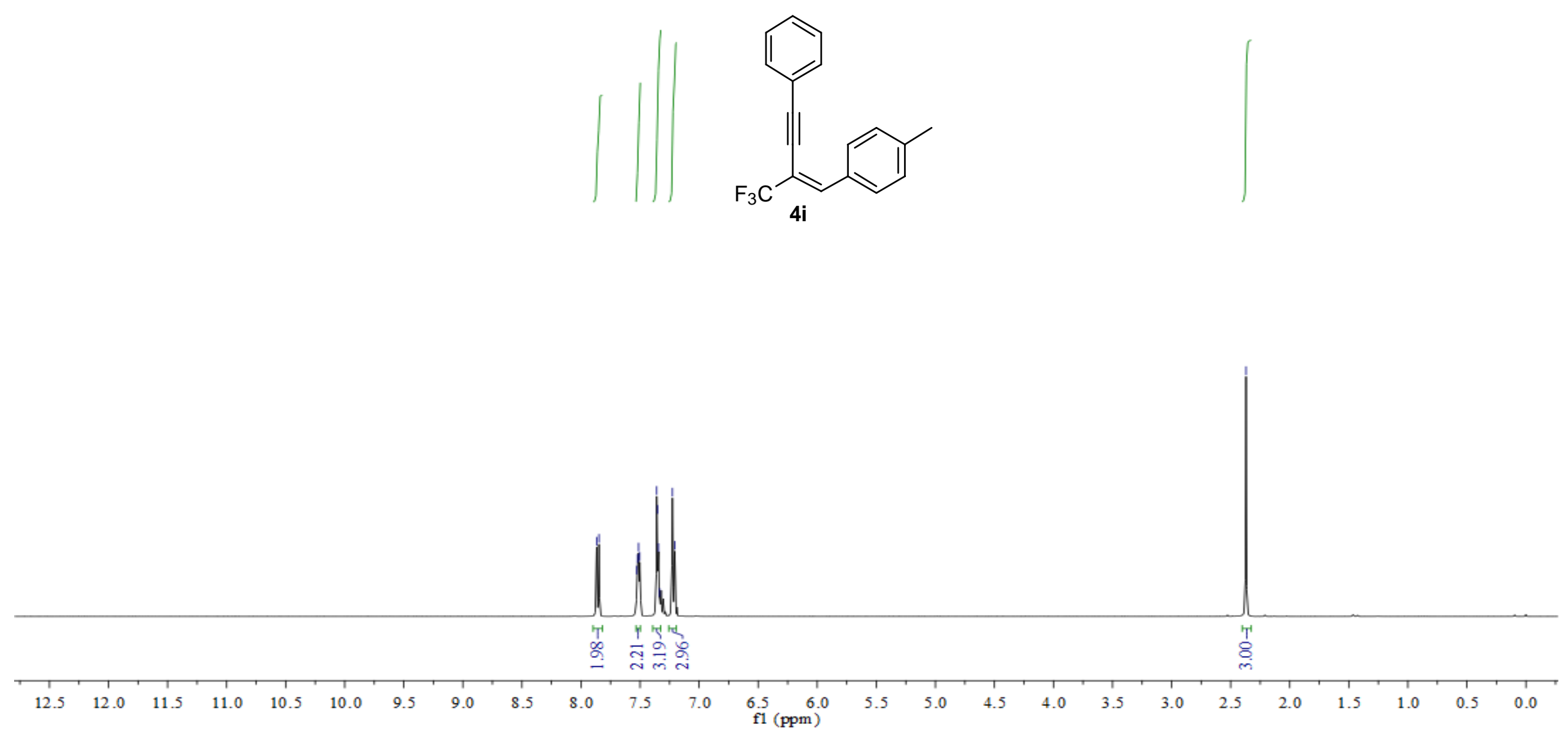


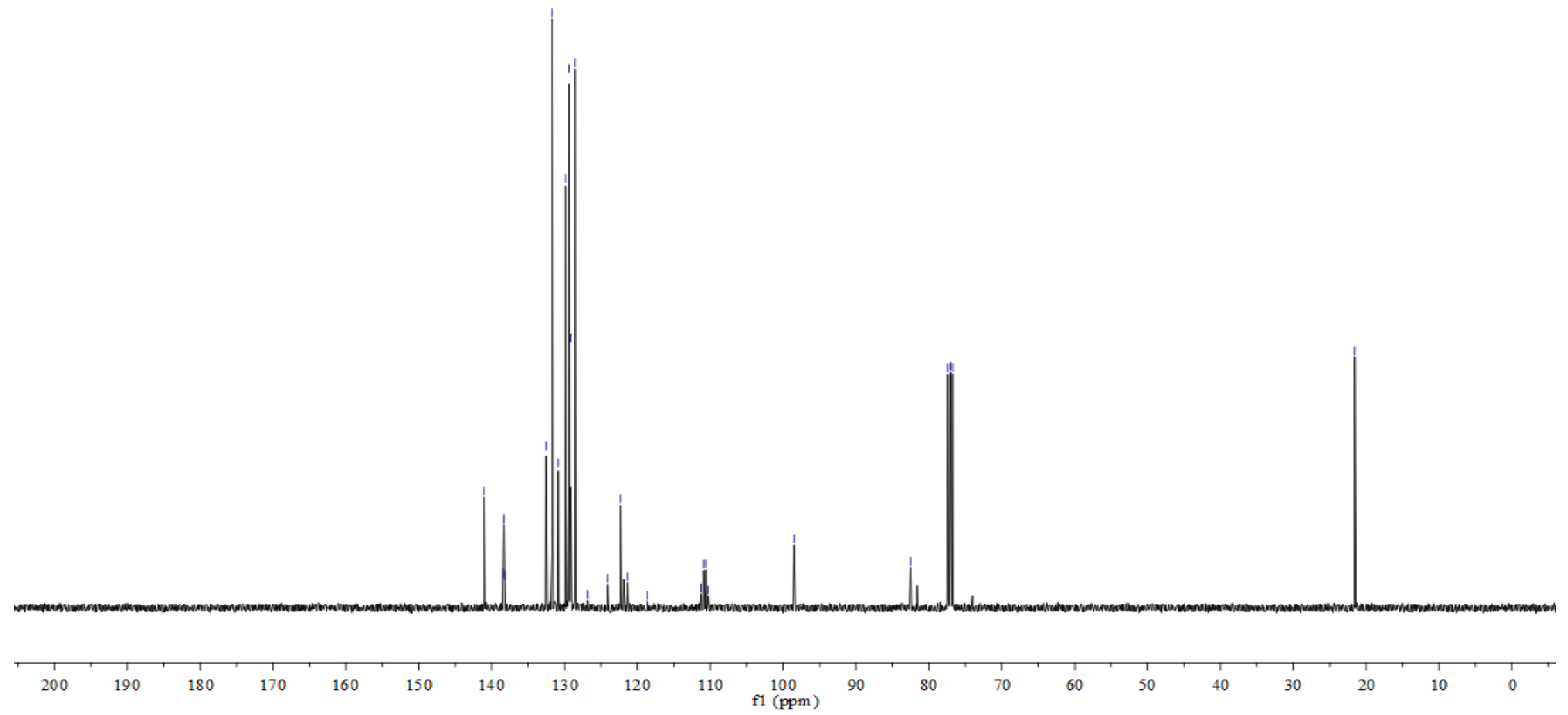


${ }^{19}$ F-NMR

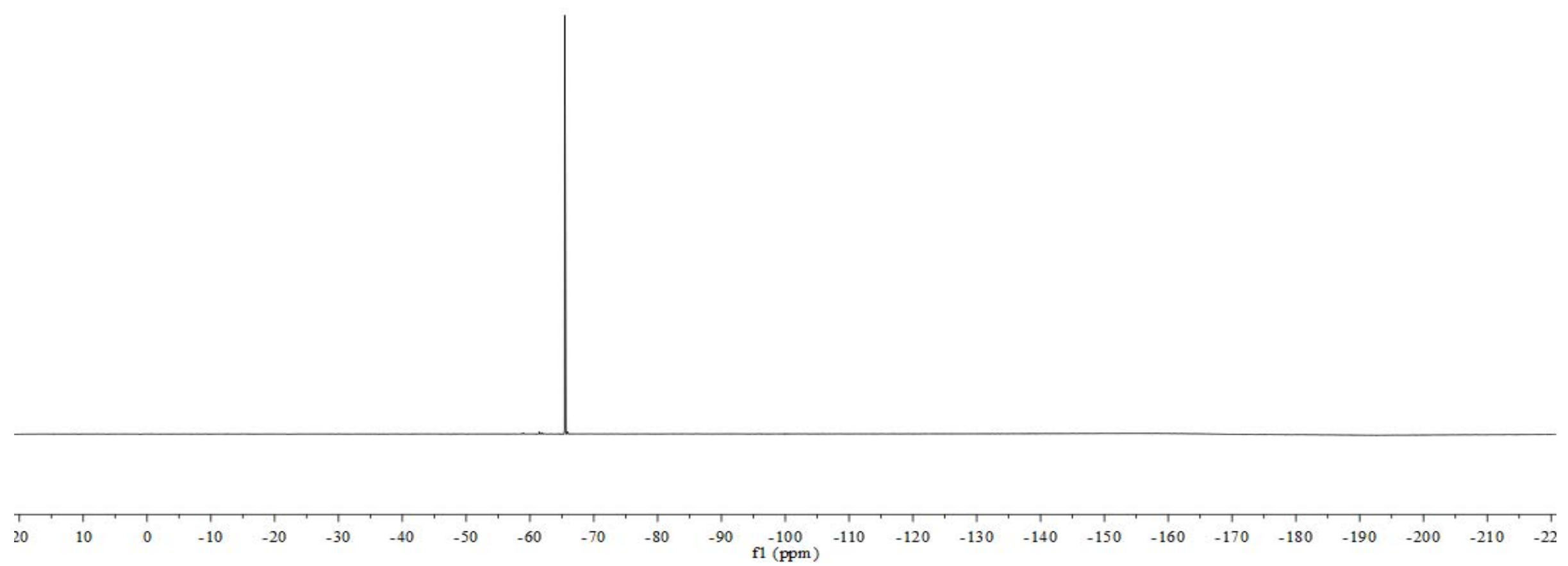



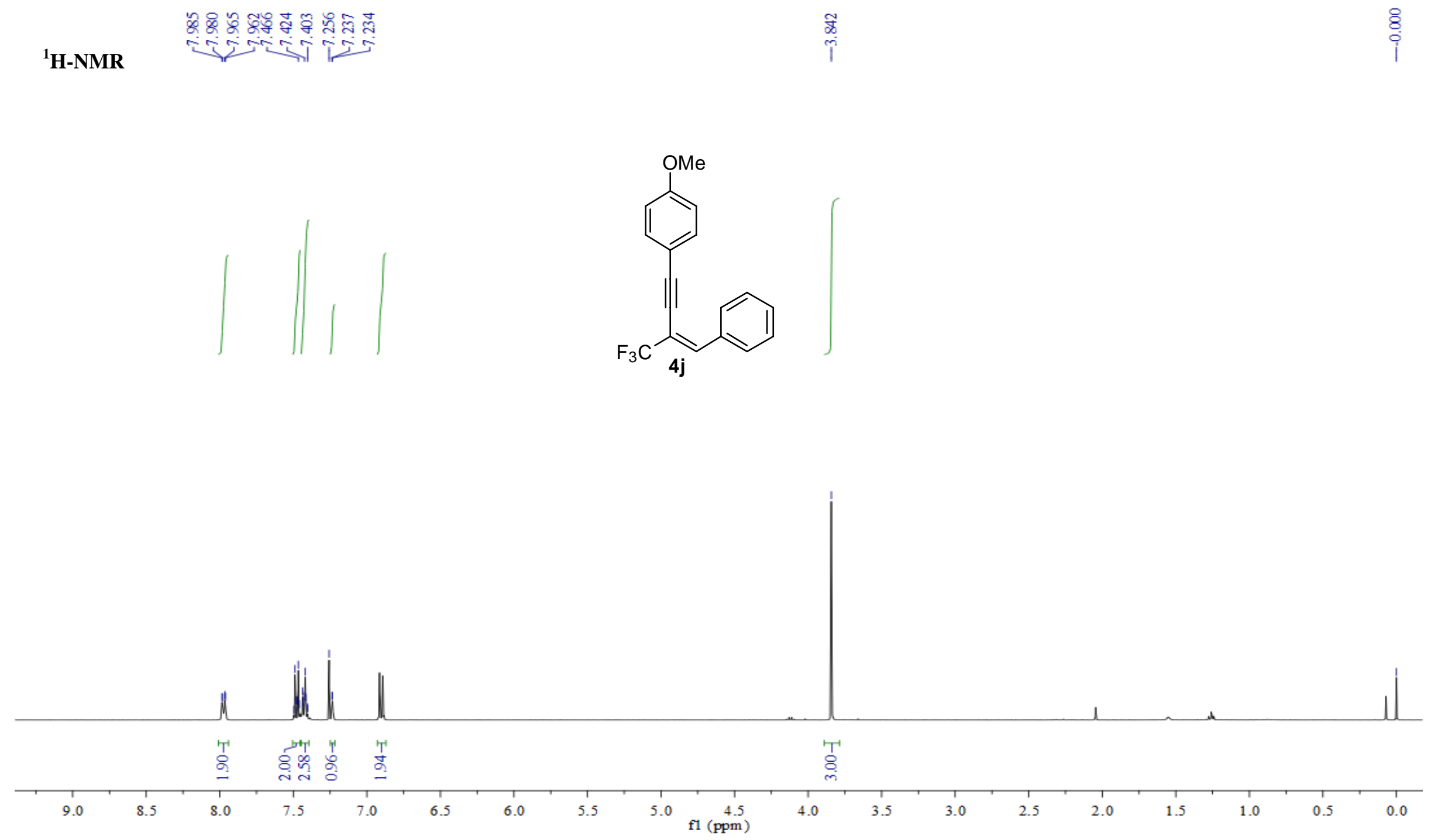


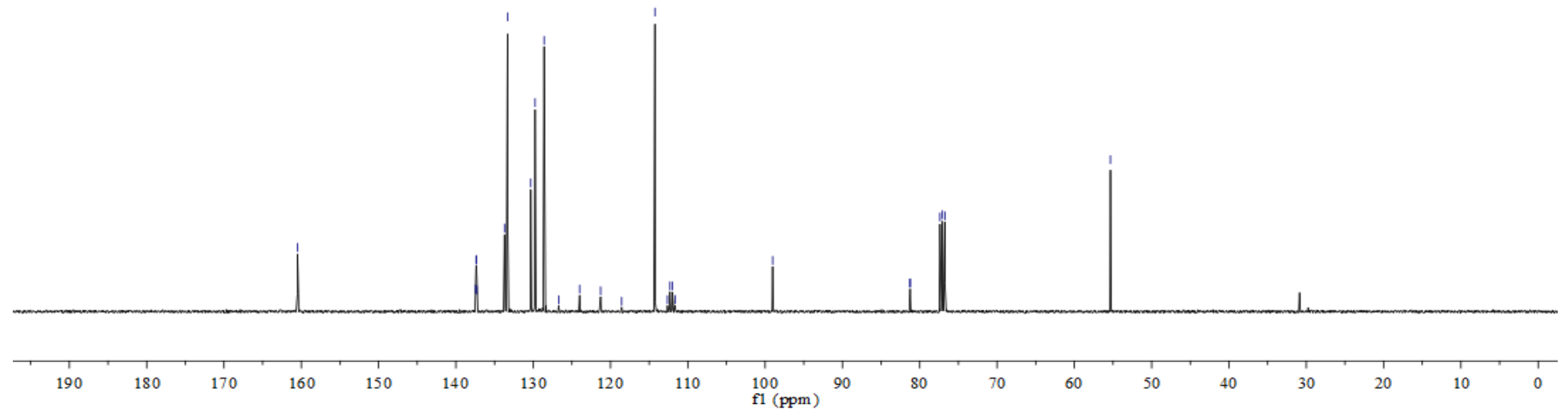


${ }^{19}$ F-NMR

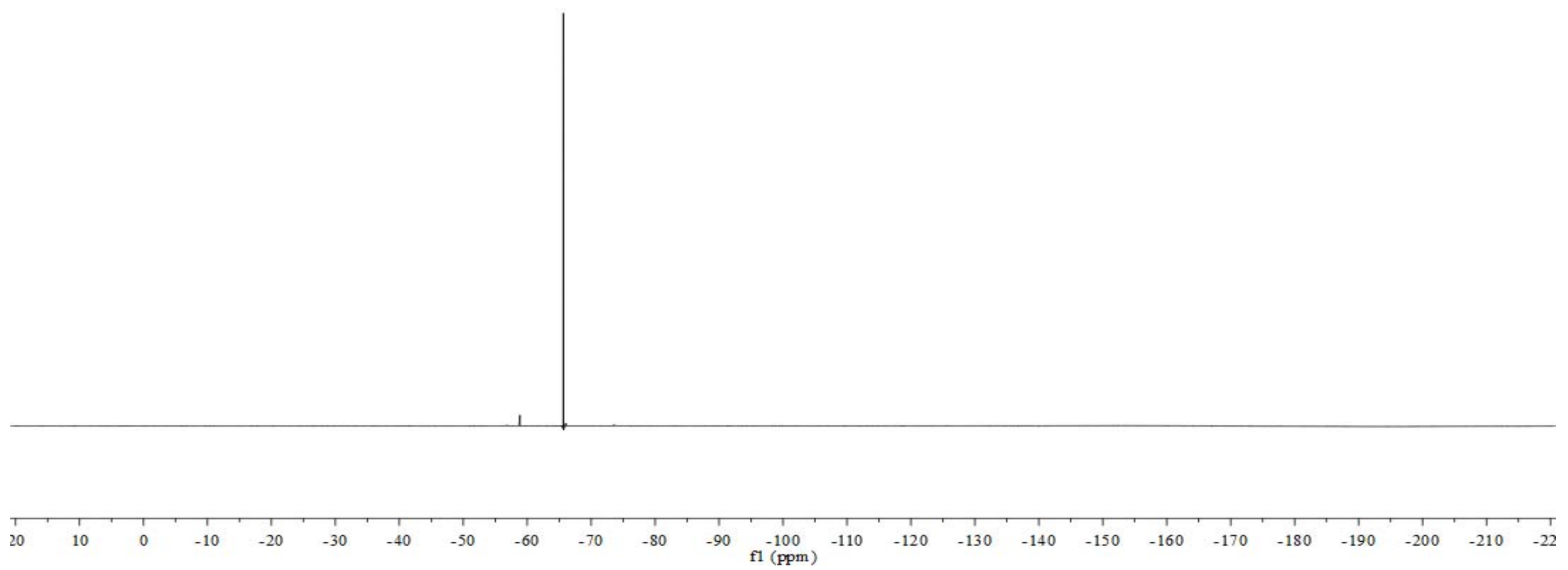




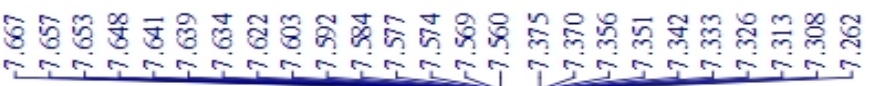

${ }^{1}$ H-NMR
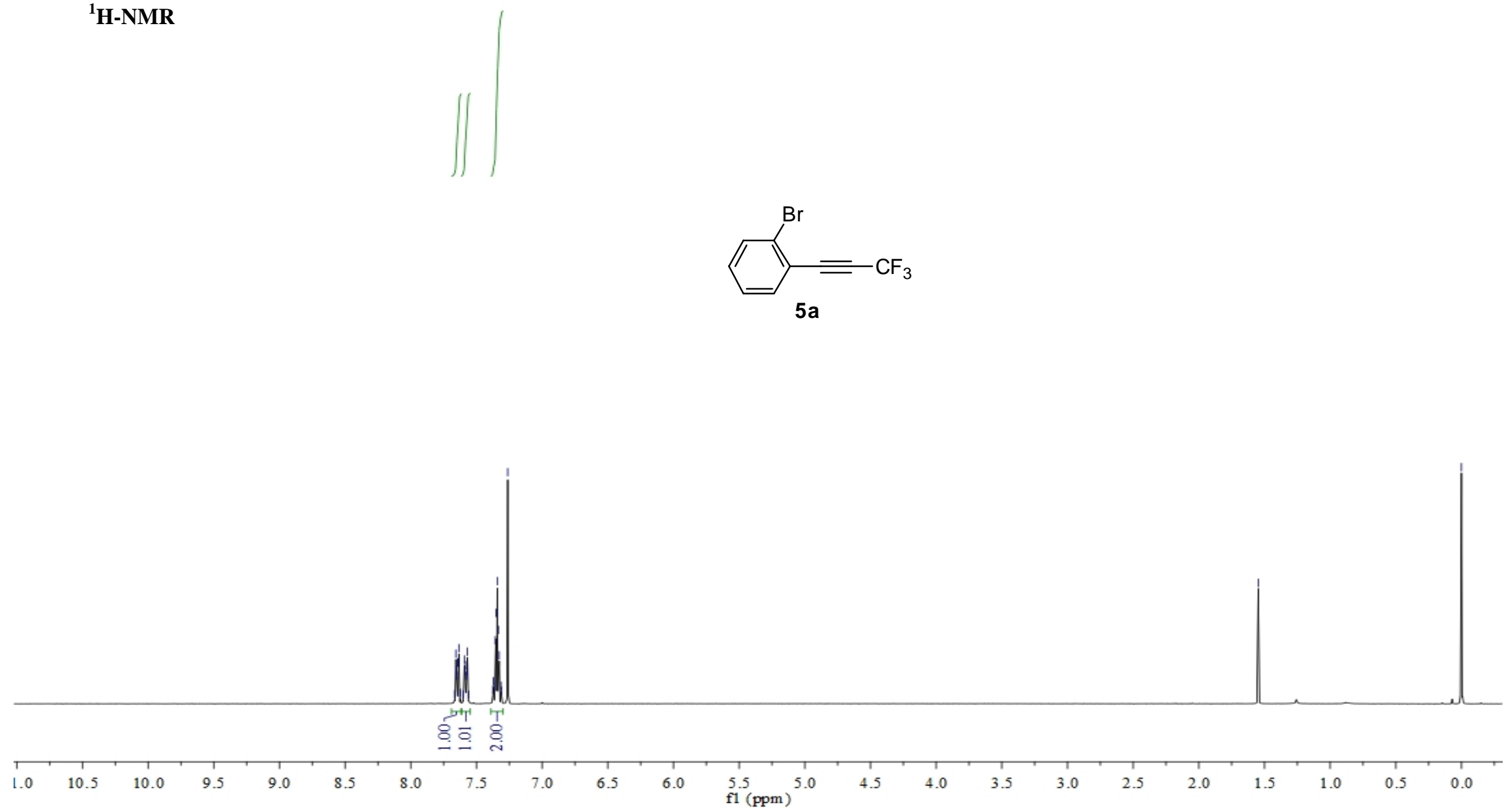


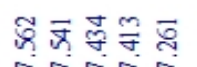

起梁T

${ }^{1}$ H-NMR

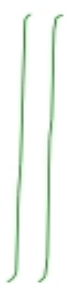

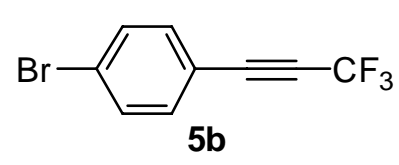

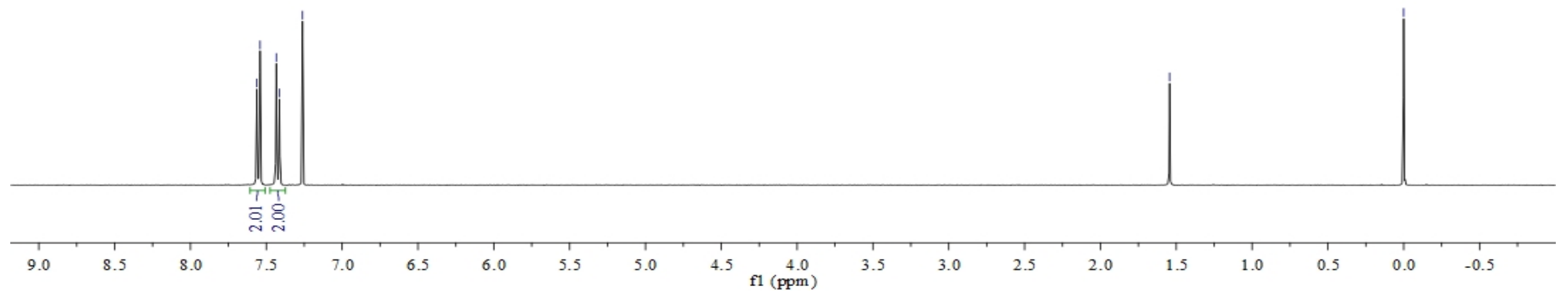


1H-NMR

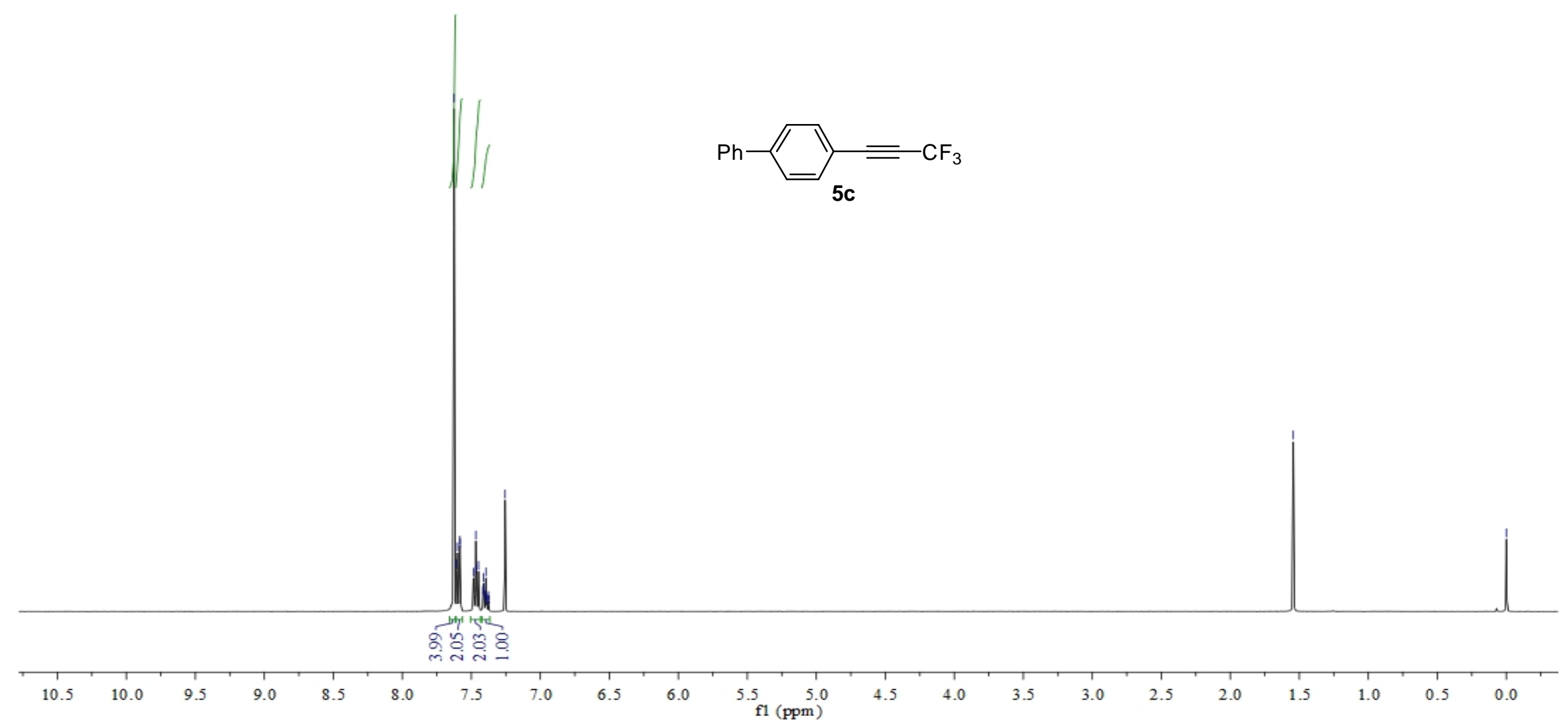




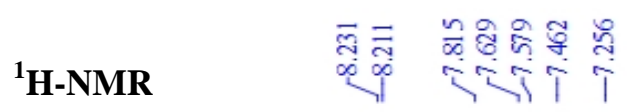
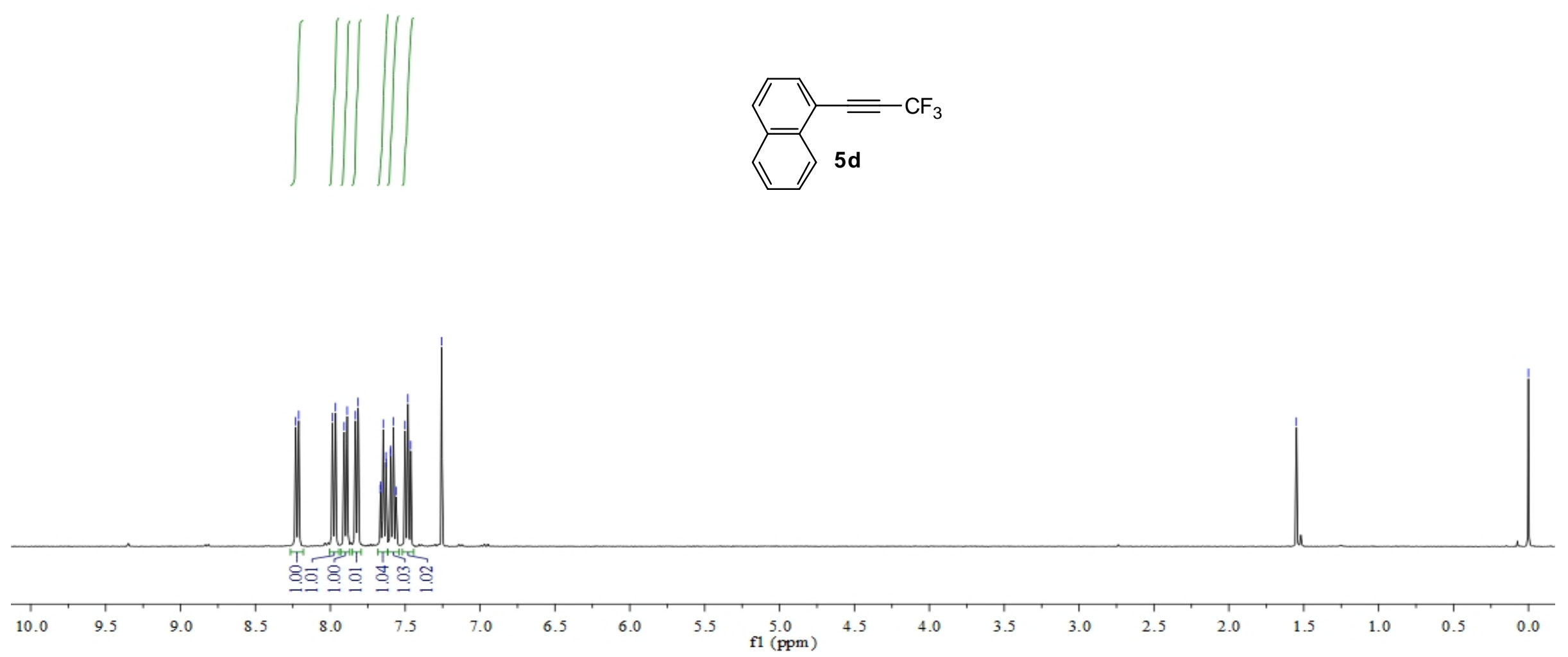
${ }^{1}$ H-NMR

ले ले సิ ते

iTis

$\sum^{\mathrm{S}} \overline{\mathrm{e}} \mathrm{CF}_{3}$

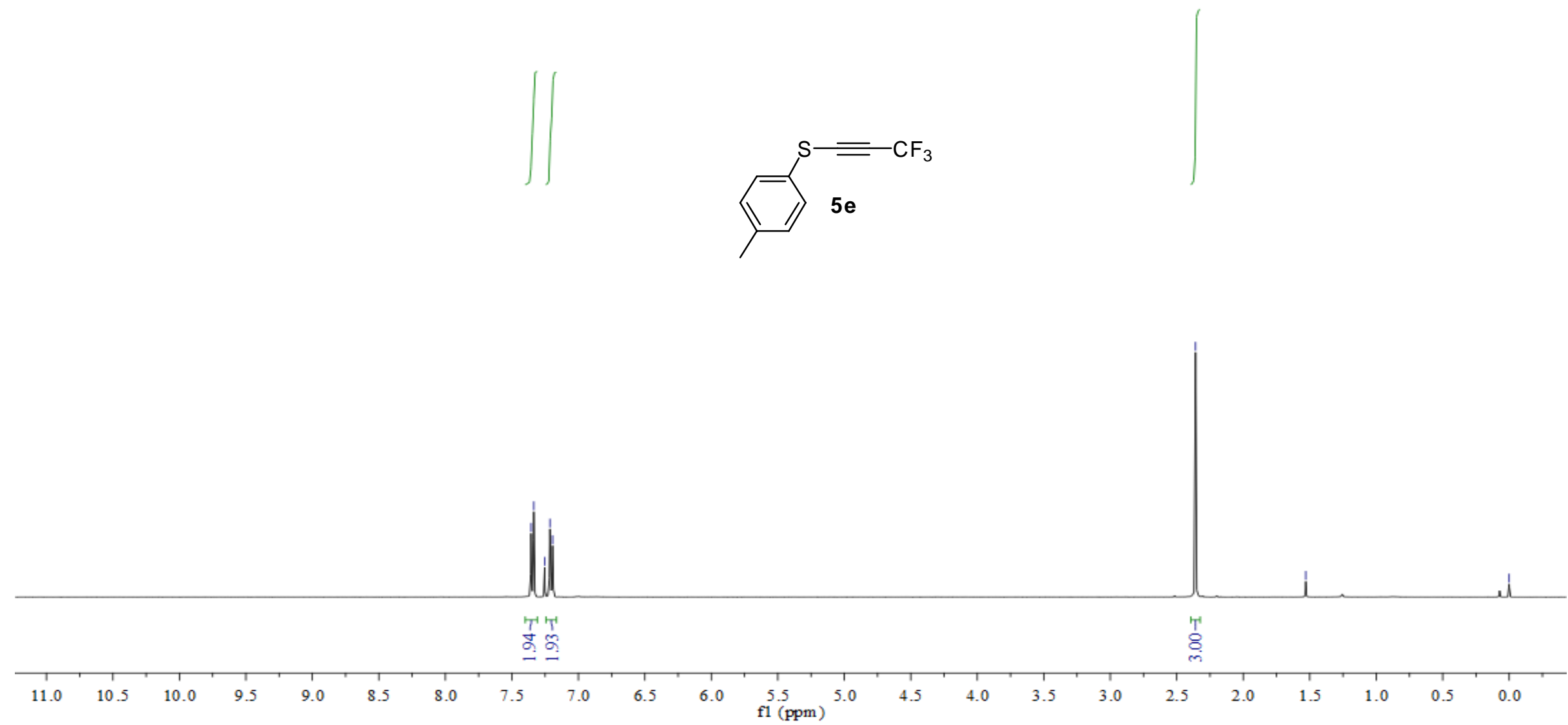




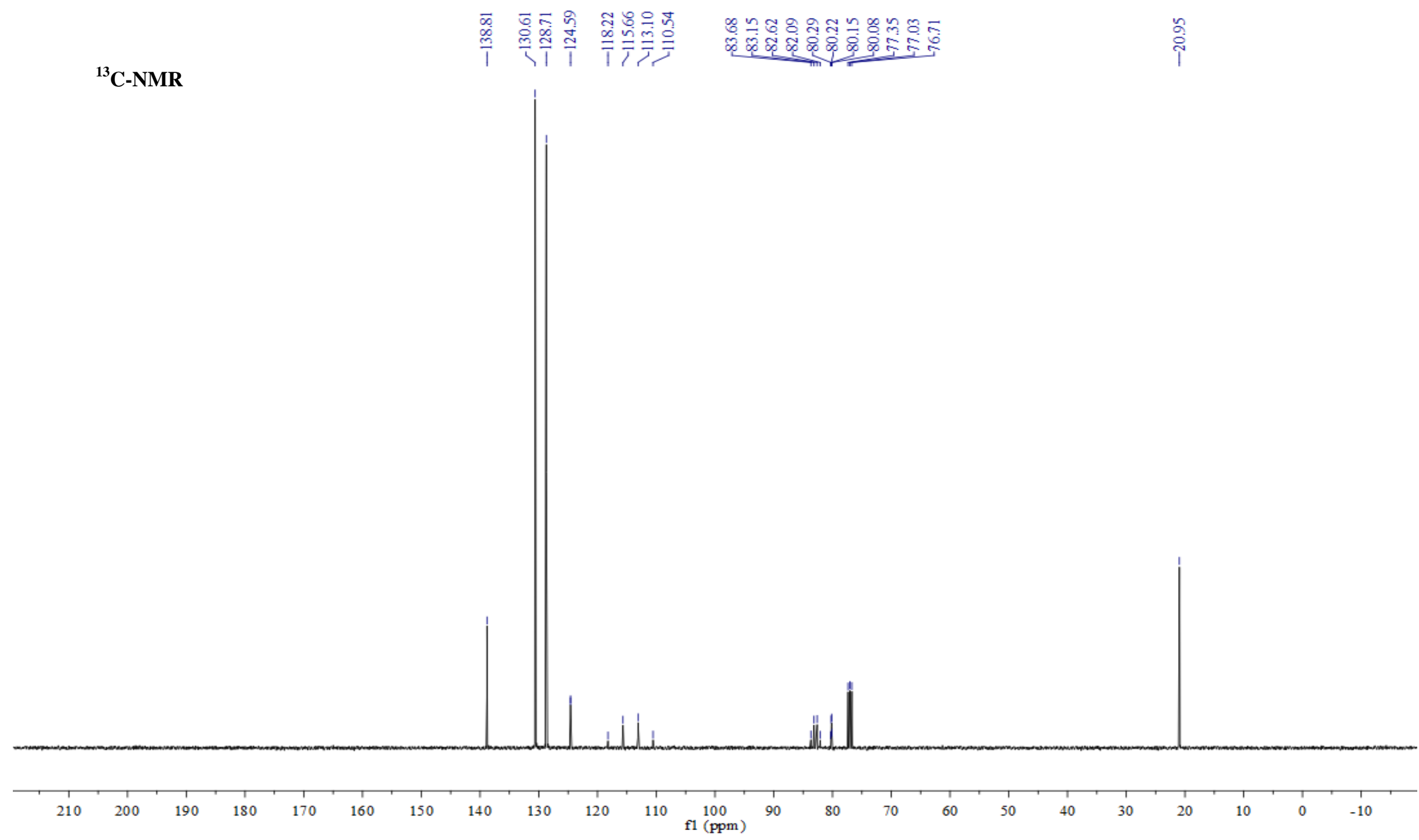


${ }^{19}$ F-NMR

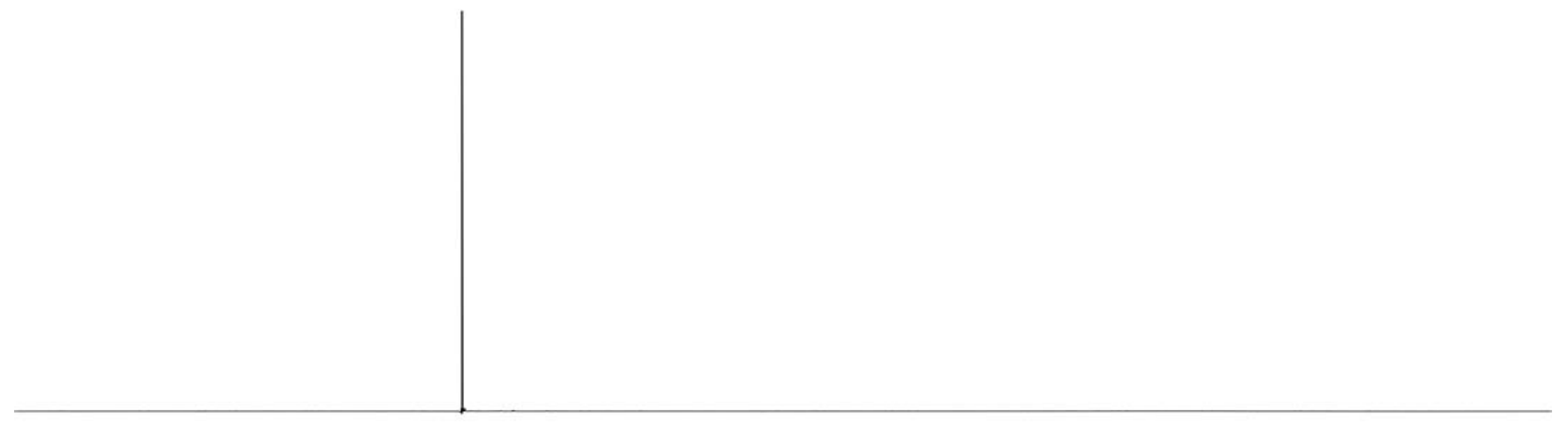

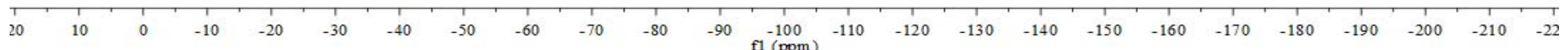



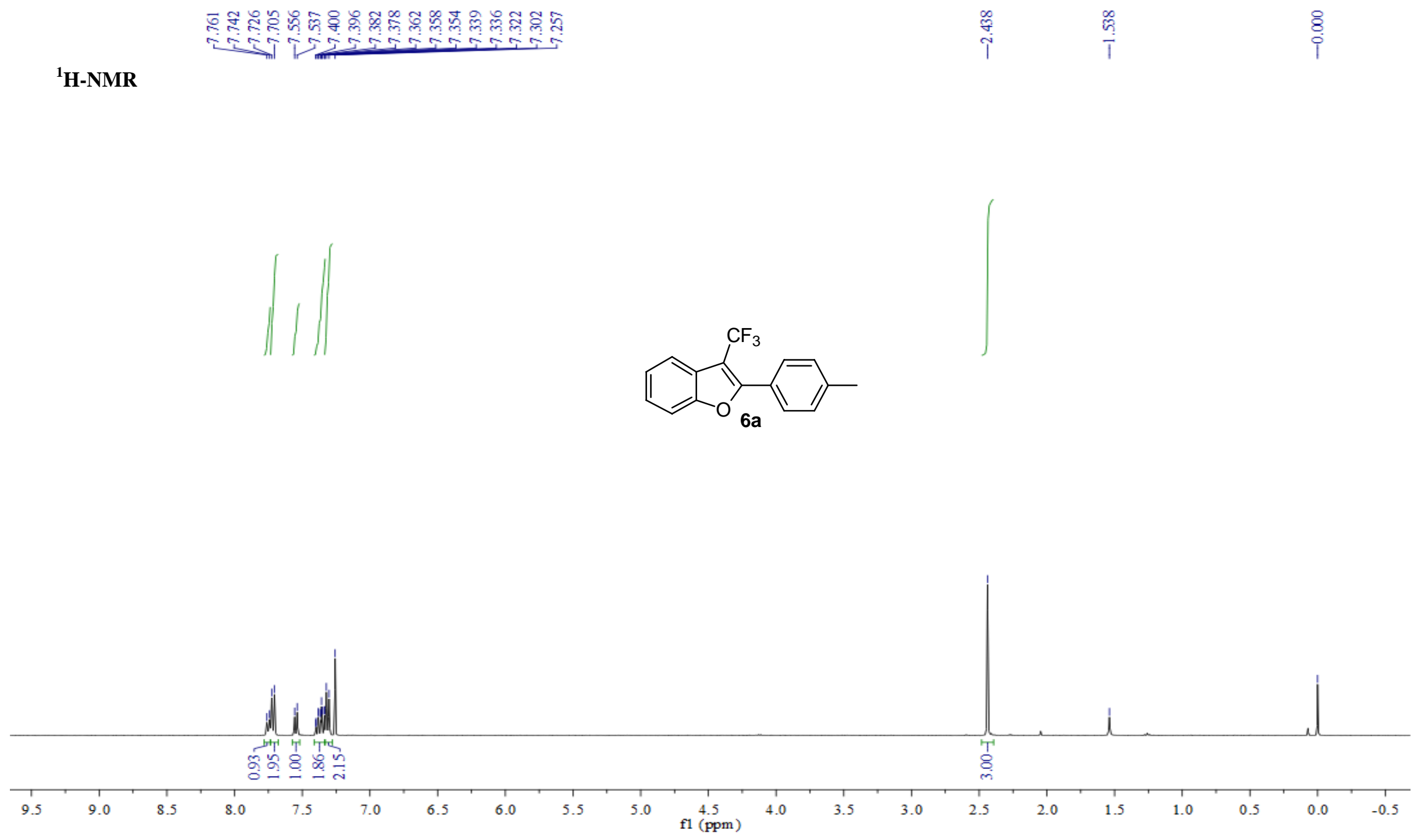


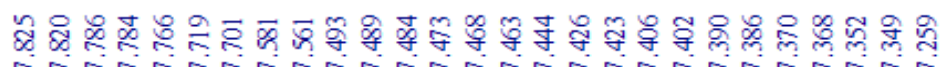

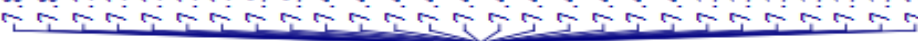

${ }^{1}$ H-NMR
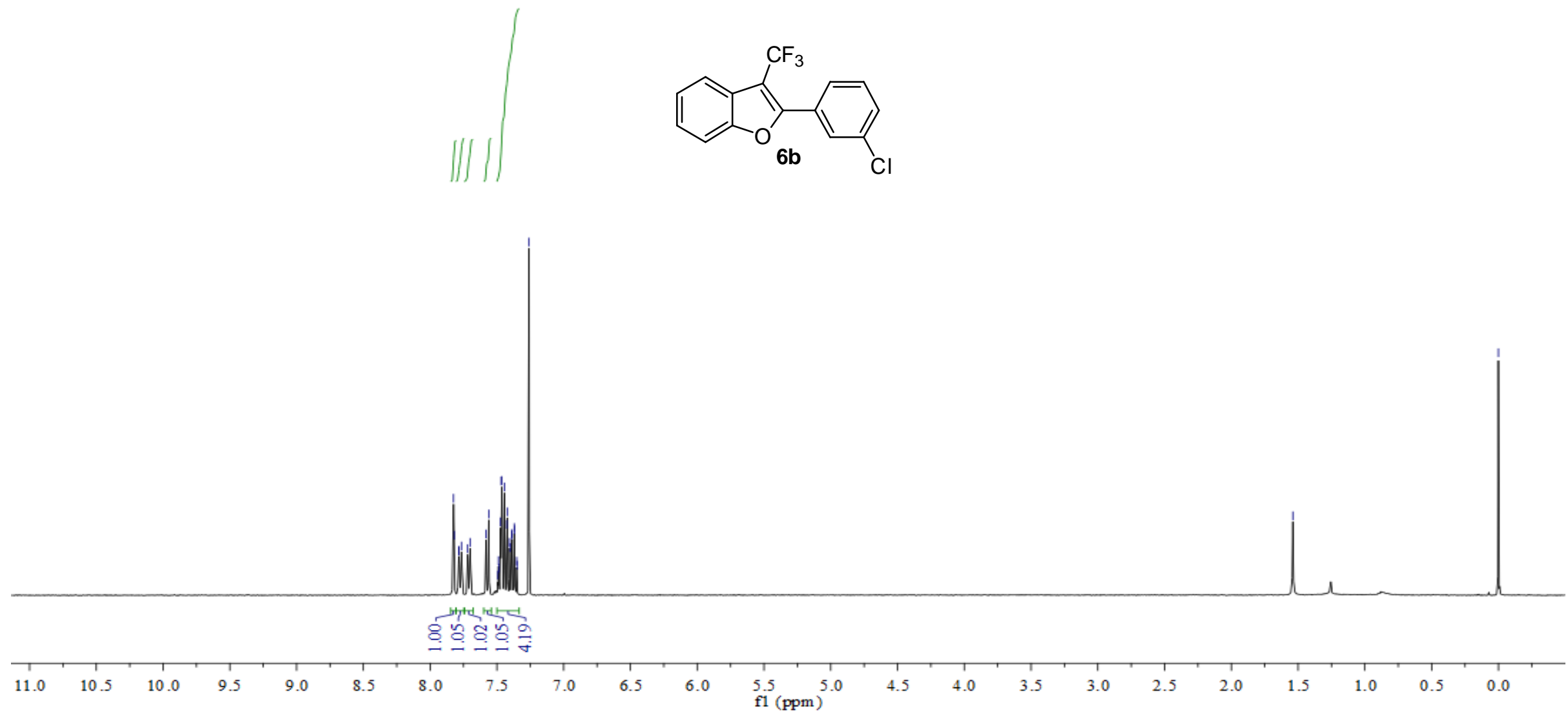
${ }^{1}$ H-NMR

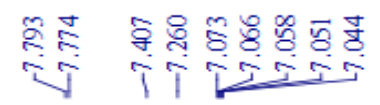
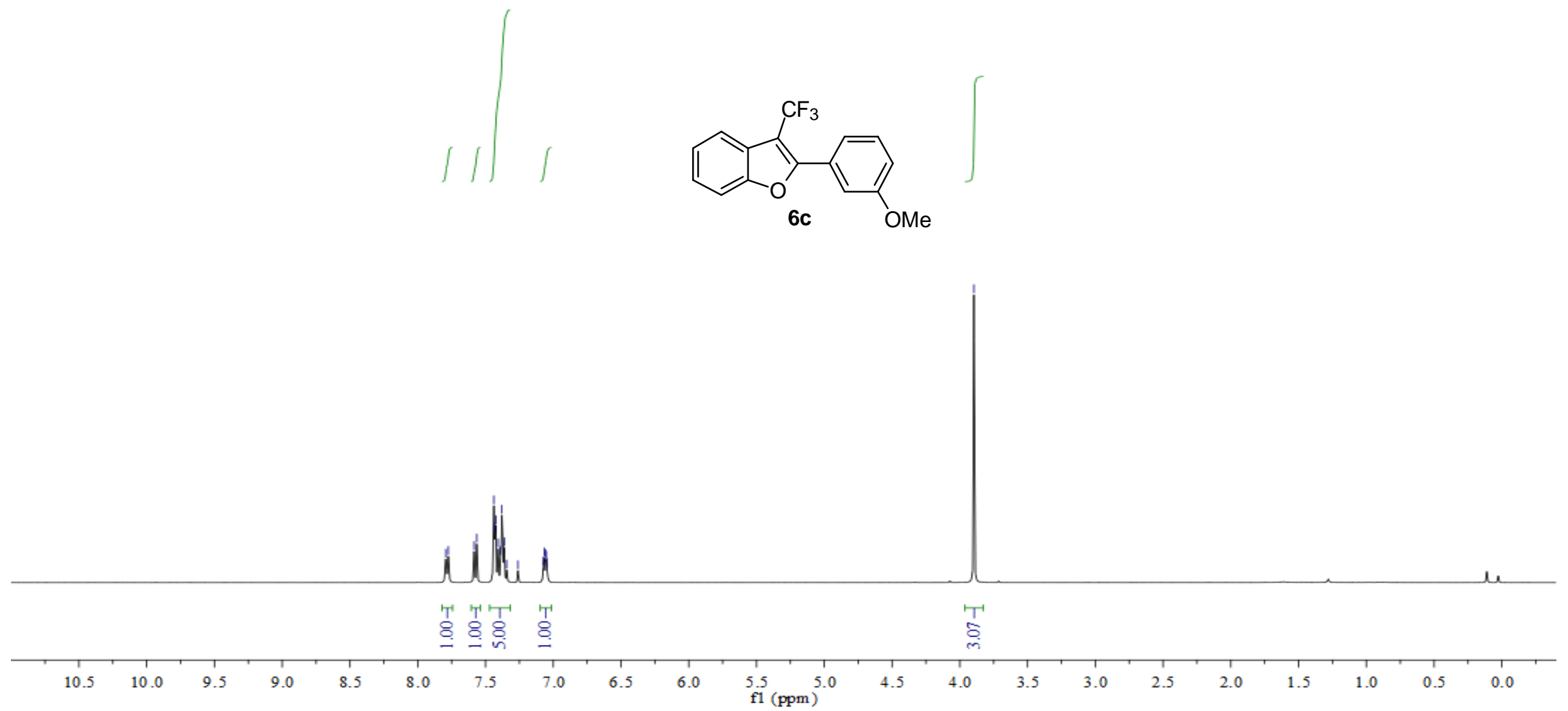

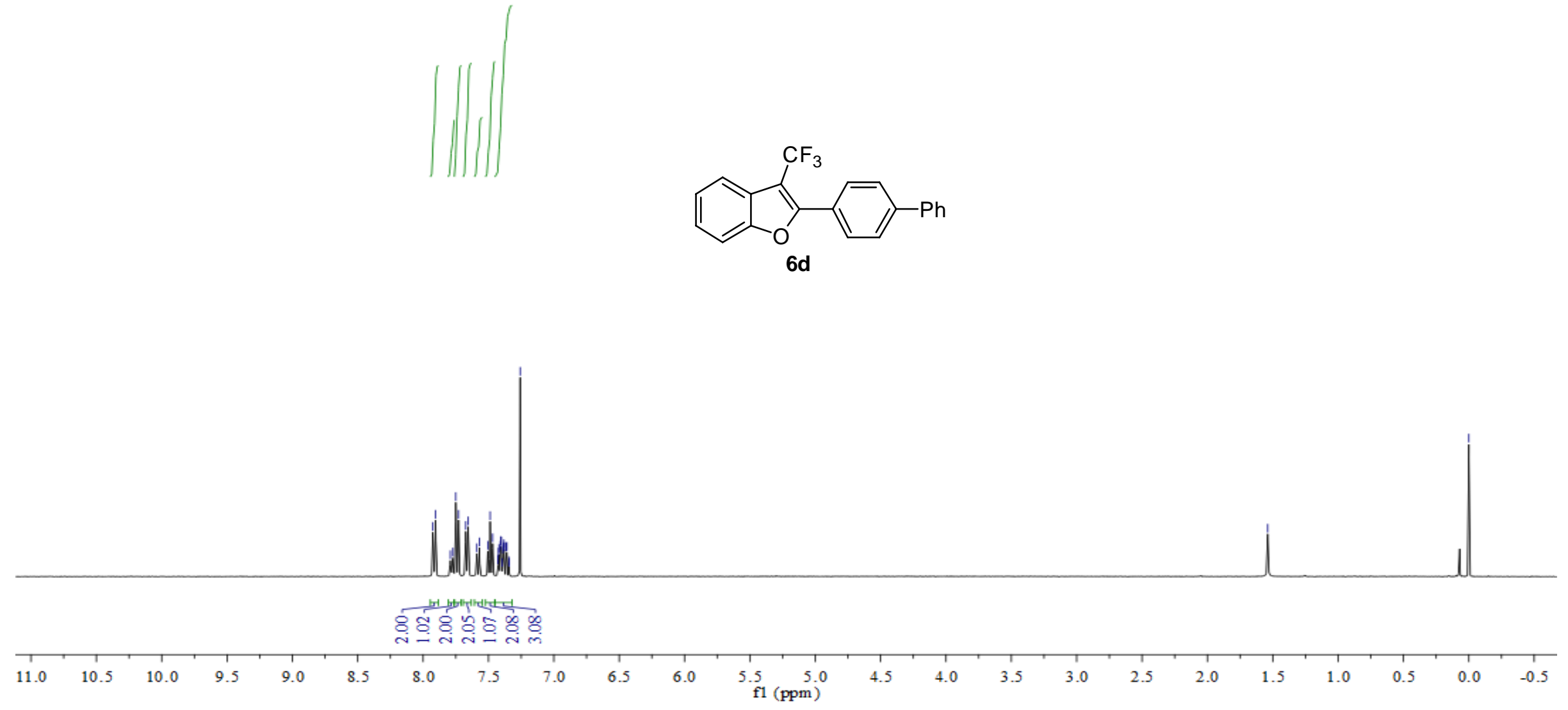


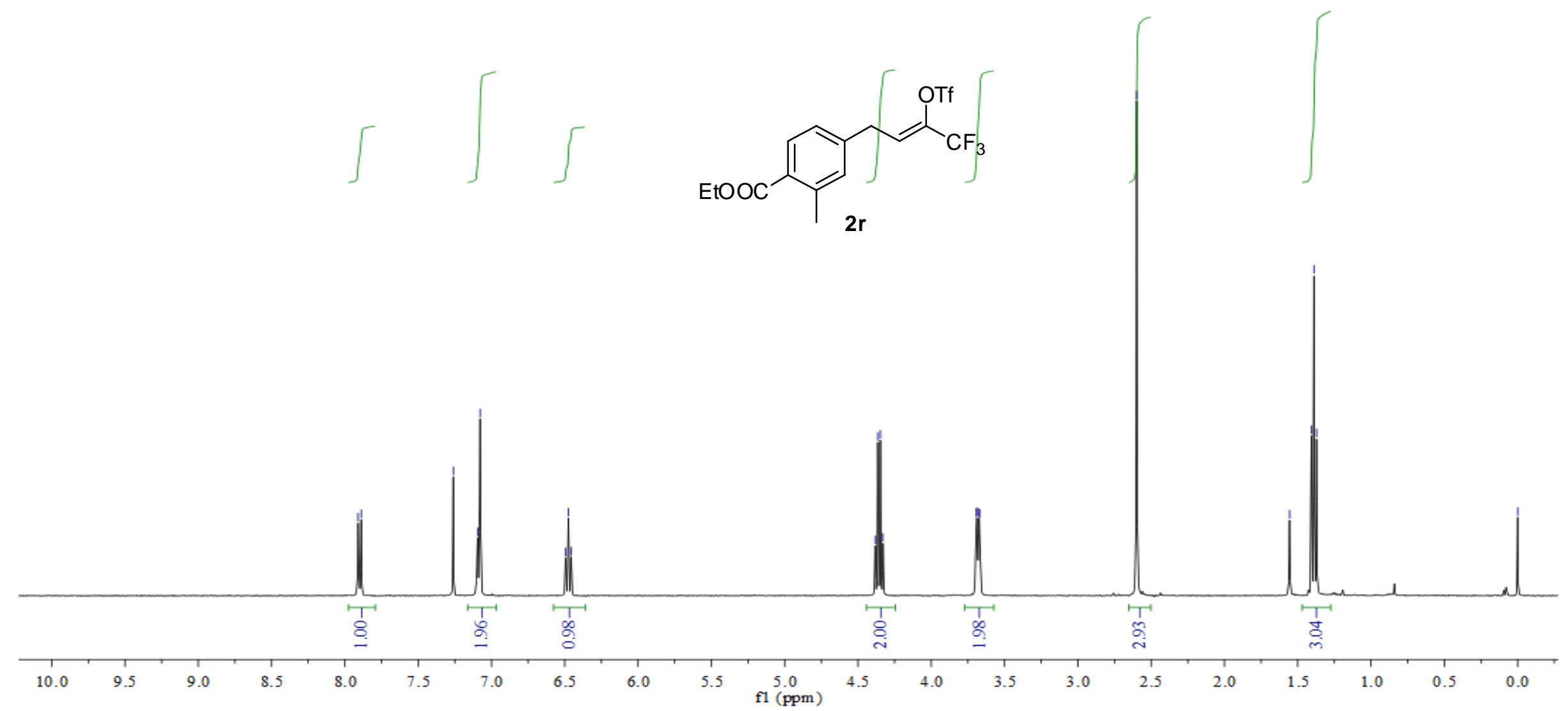




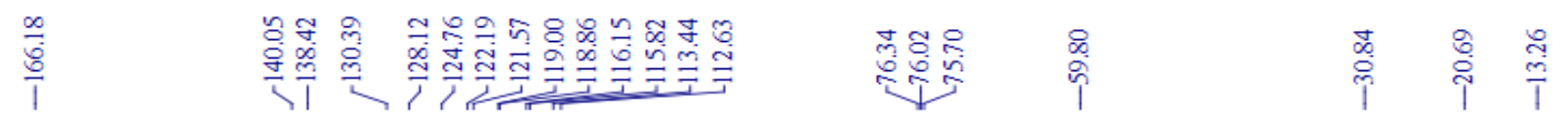

${ }^{13}$ C-NMR

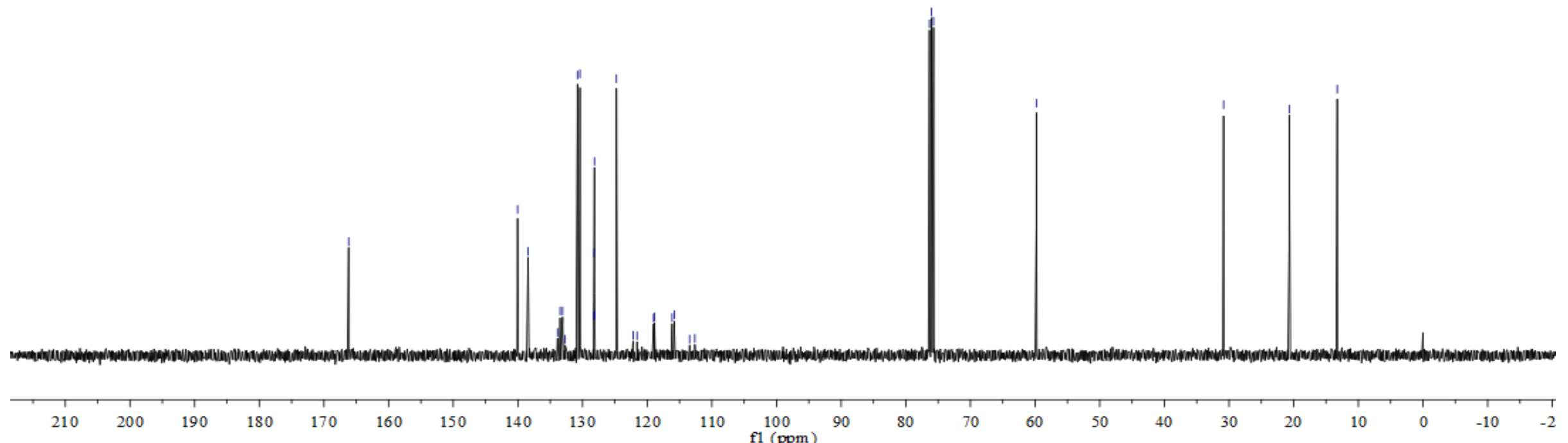


${ }^{19}$ F-NMR

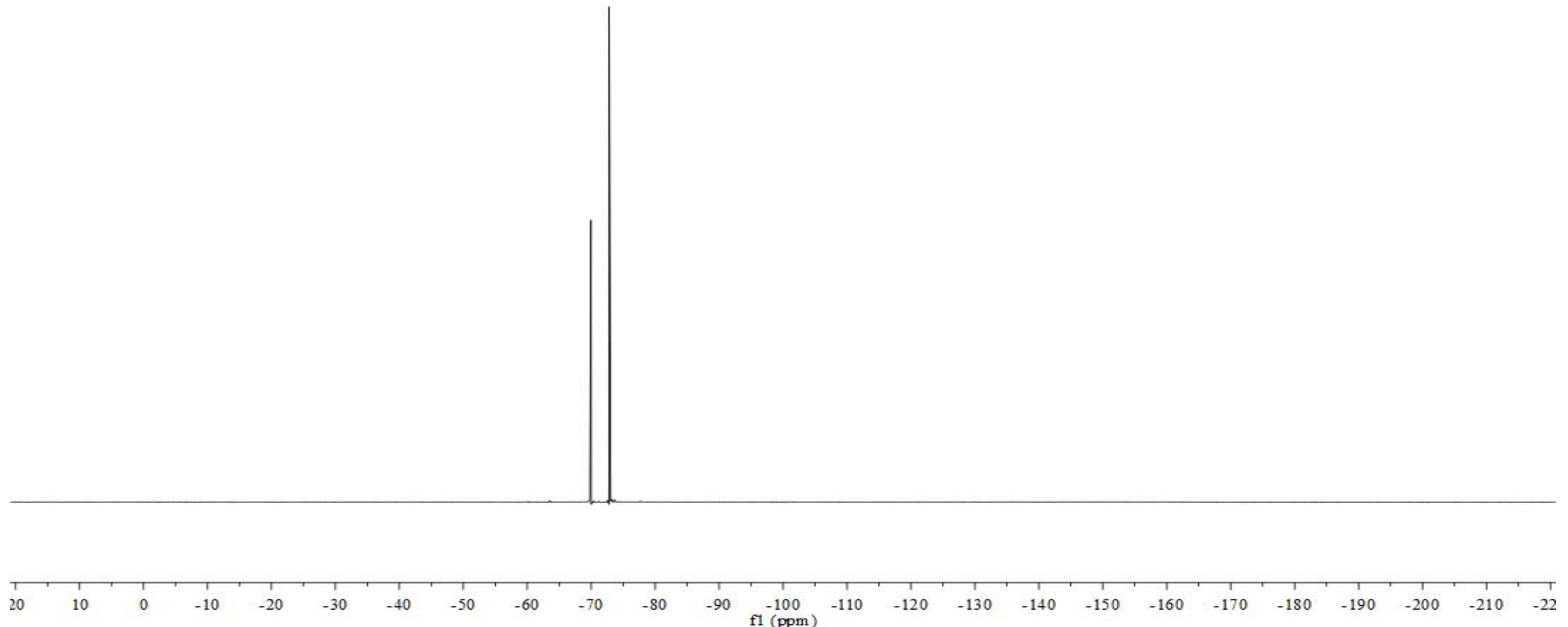




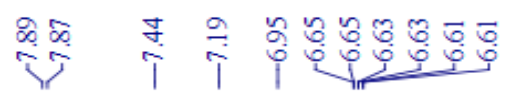

${ }^{1}$ H-NMR

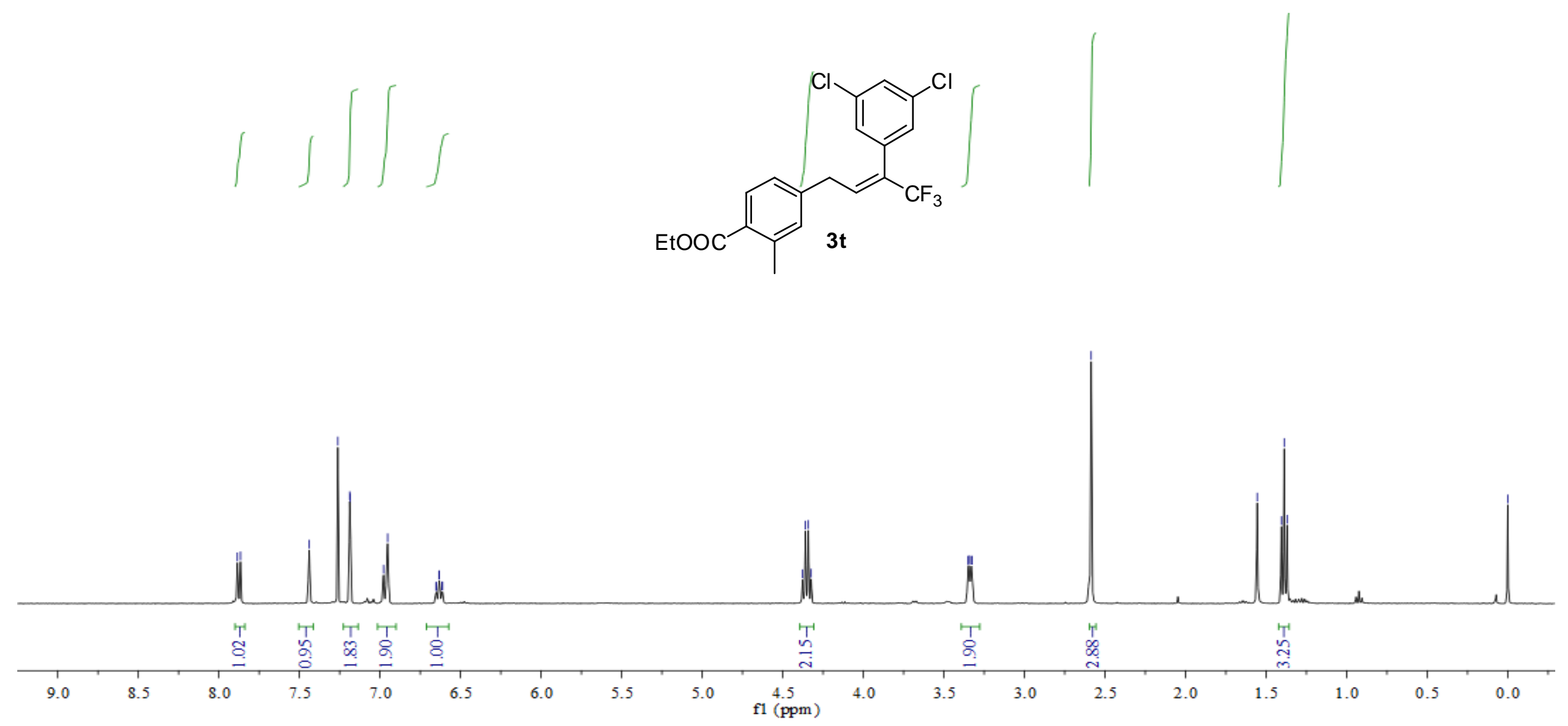

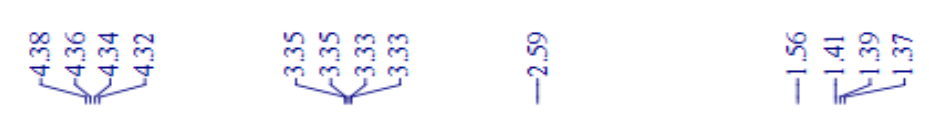

$\stackrel{8}{0}$ 


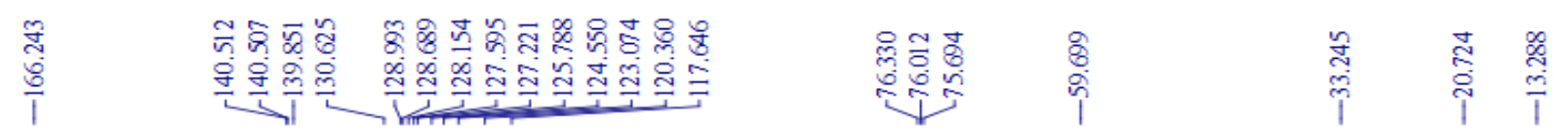

${ }^{13}$ C-NMR

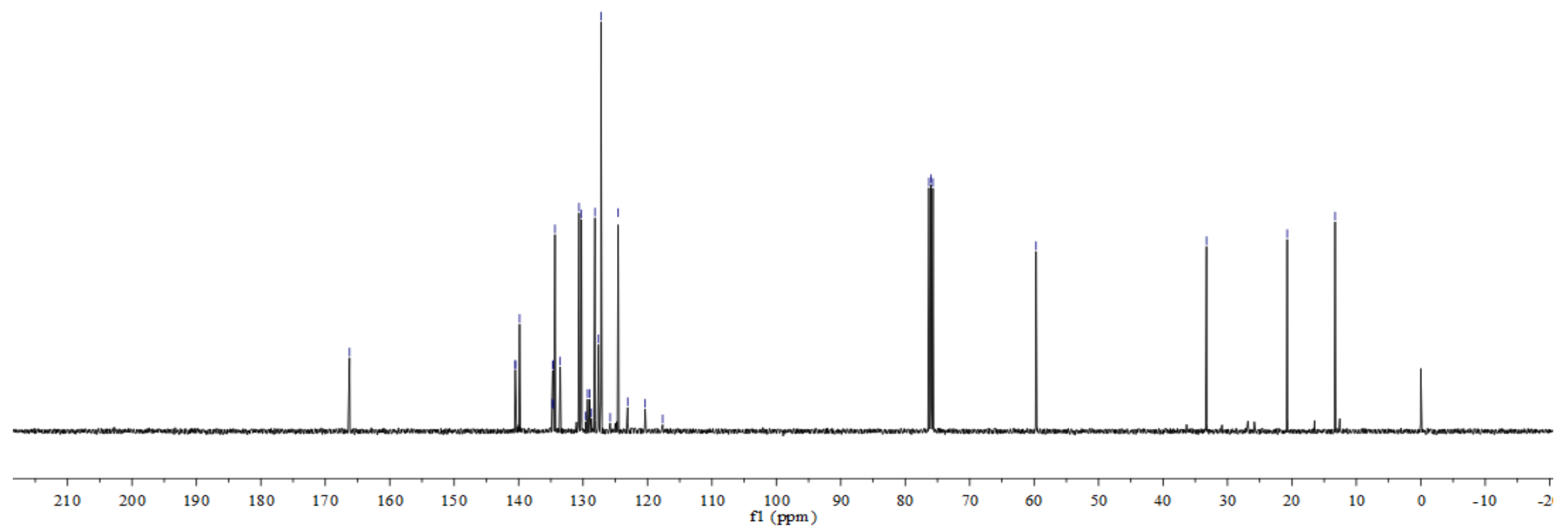


${ }^{19}$ F-NMR

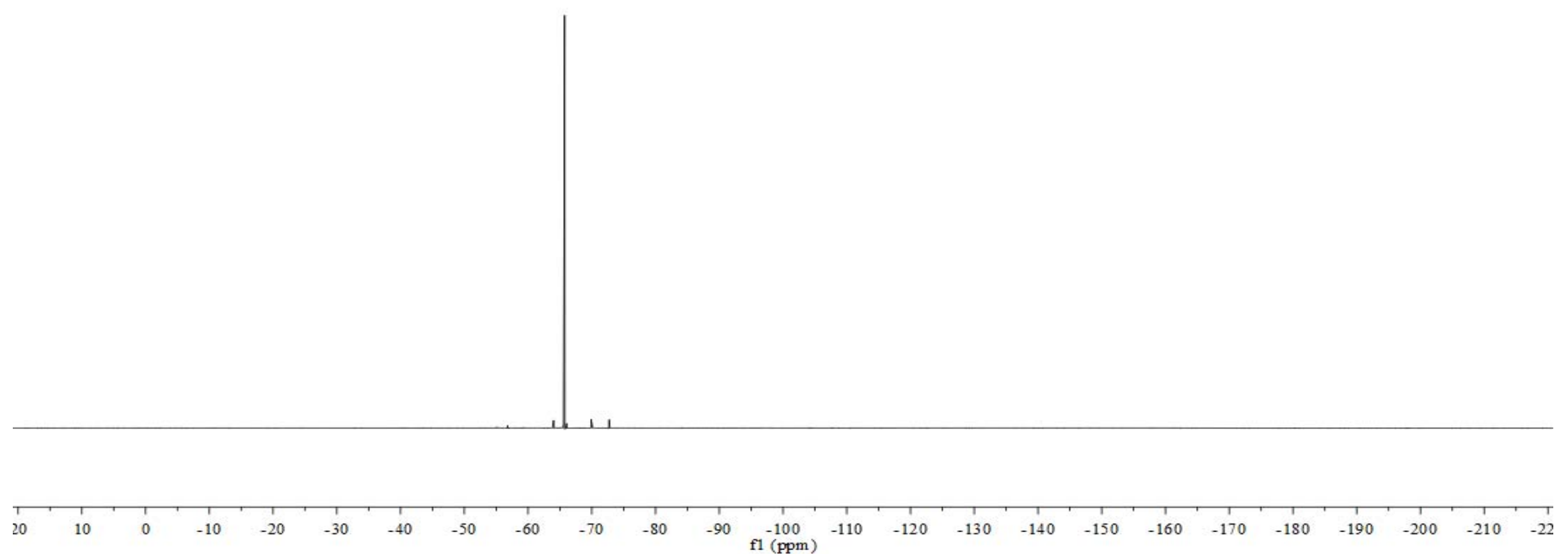



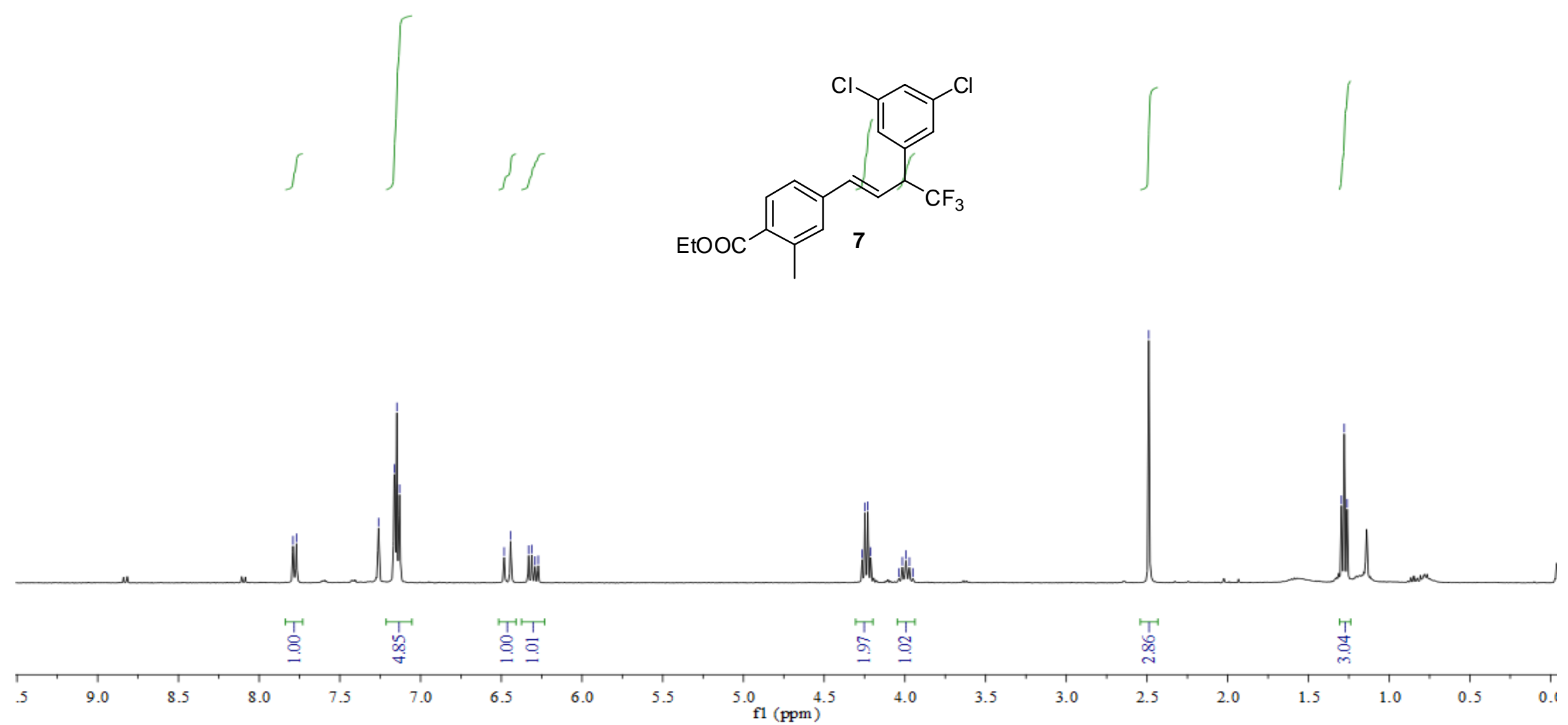


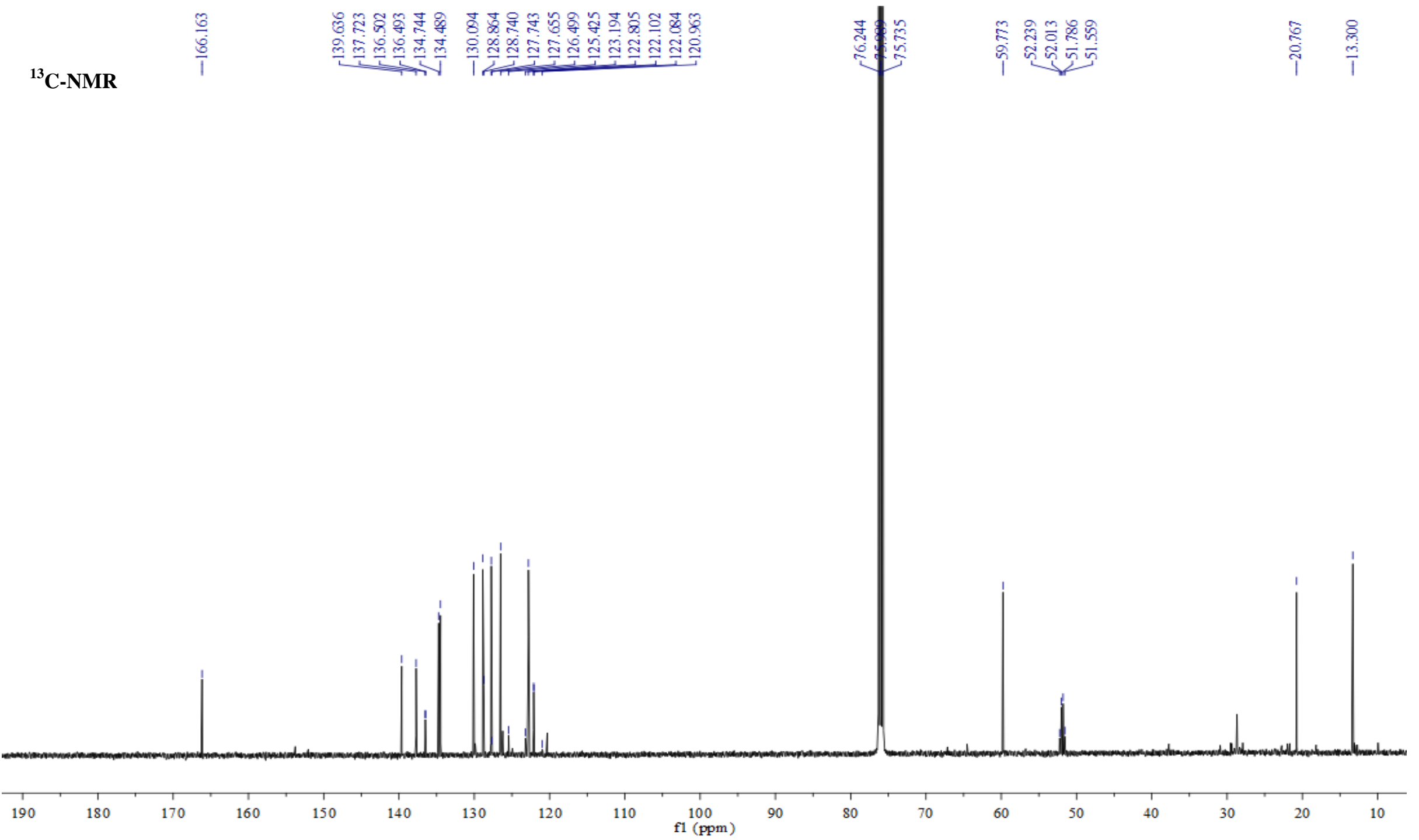


${ }^{19}$ F-NMR

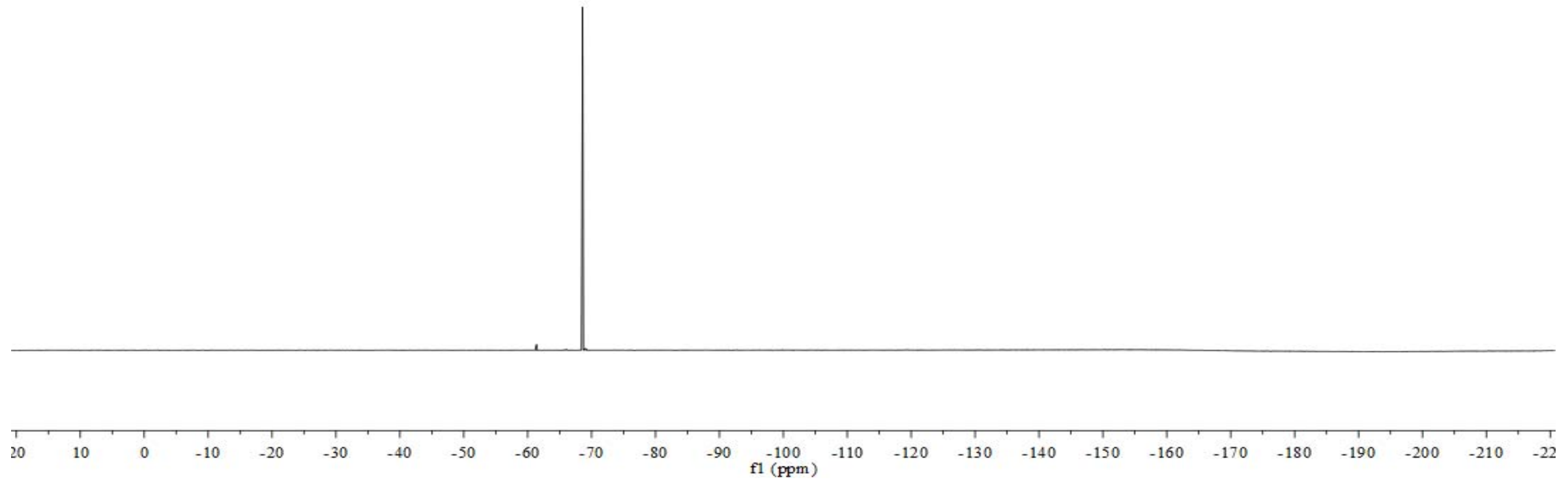

